\title{
DCM3D: A Dual-Continuum, Three-Dimensional, Ground-Water Flow Code for Unsaturated, Fractured, Porous Media
}

Manuscript Completed: January 1991

Date Published: February 1991

Prepared by

C. D. Updegraff, GRAM, Inc.

C. E. Lee, Applied Physics, Inc.

D. P. Gallegos, Sandia National Laboratories

GRAM, Inc.

1709 Moon Street NE

Albuquerque, NM 87112

Applied Physics, Inc.

5353 Wyoming Blvd. NE

Albuquerque, NM 87109

Under Contract to:

Sandia National Laboratories

Albuquerque, NM 87185

\section{Prepared for}

Division of Engineering

Office of Nuclear Regulatory Research

U.S. Nuclear Regulatory Commission

Washington, DC 20555

NRC FIN A1266 


\section{DISCLAIMER}

This report was prepared as an account of work sponsored by an agency of the United States Government. Neither the United States Government nor any agency Thereof, nor any of their employees, makes any warranty, express or implied, or assumes any legal liability or responsibility for the accuracy, completeness, or usefulness of any information, apparatus, product, or process disclosed, or represents that its use would not infringe privately owned rights. Reference herein to any specific commercial product, process, or service by trade name, trademark, manufacturer, or otherwise does not necessarily constitute or imply its endorsement, recommendation, or favoring by the United States Government or any agency thereof. The views and opinions of authors expressed herein do not necessarily state or reflect those of the United States Government or any agency thereof. 


\section{DISCLAIMER}

Portions of this document may be illegible in electronic image products. Images are produced from the best available original document. 


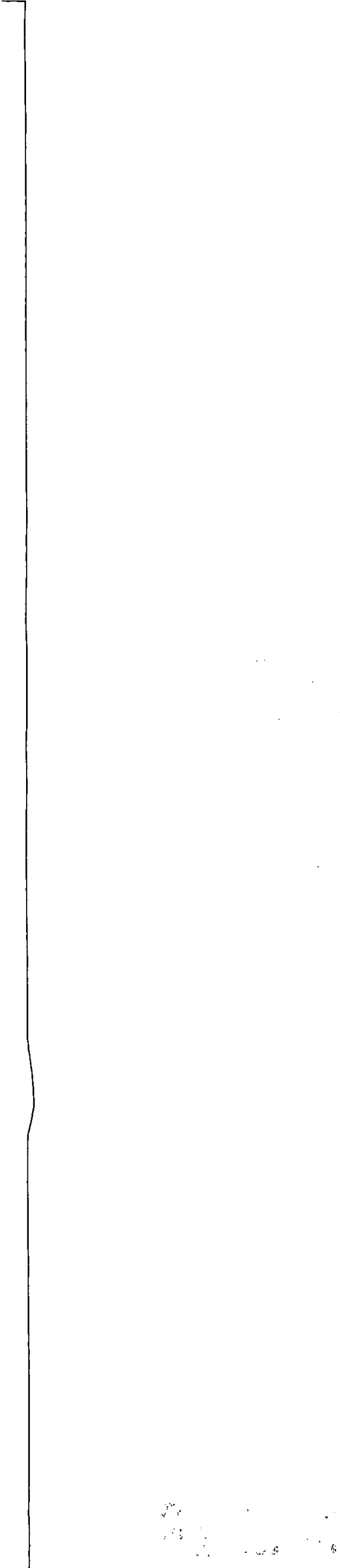

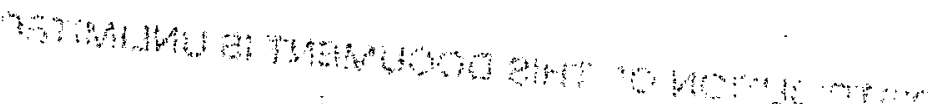

$$
\therefore \text {. }
$$

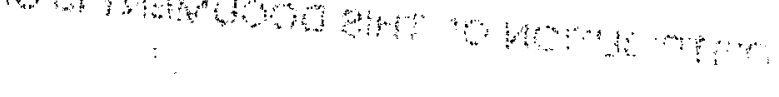




\section{ABSTRACT}

This report constitutes the user's manual for DCM3D. DCM3D is a computer code for solving three-dimensional, ground-water flow problems in variably saturated, fractured porous media. The code is based on a dual-continuum model with porous media comprising one continuum and fractures comprising the other. The continua are connected by a transfer term that depends on the unsaturated permeability of the porous medium. An integrated finite-difference scheme is used to discretize the governing equations in space. The time-dependent term is allowed to remain continuous. The resulting set of ordinary differential equations (ODE's) is solved with a general ODE solver, LSODES. The code is capable of handling transient, spatially dependent source terms and boundary conditions. The boundary conditions can be either prescribed head or prescribed flux. 


\section{CONTENTS}

1.0 INTRODUCTION

1.1 Overview 1

1.2 Background and objectives 1

1.3 Purpose and Need 1

1.4 Organization 2

2.0 FLOW IN AN UNSATURATED, FRACTURED, POROUS MEDIUM 3

2.1 Background 3

2.2 Assumptions 4

2.2.1 Double Continuum Model 4

2.2.2 Darcy's Law 5

2.2.3 Van Genuchten Characteristic Curves 6

2.2.4 Other Assumptions 7

2.3 Flow Equations 8

2.4 Transfer Term 10

3.0 NUMERICAL IMPLEMENTATION 13

3.1 Flow Model Difference Equations Development 13

3.2 Finite Difference Equations 16

3.3 External Boundary Conditions 23

3.3.1 Flux Boundary Conditions 23

3.3.2 Pressure Boundary Conditions 24

3.3.3 Time Dependent Volume Sources and Boundary Conditions 26

4.0 CODE STRUCTURE 29

4.1 Main Driver 29

4.2 General Description of the LSODES ODE Solver 38

5.0 INSTRUCTIONS FOR RUNNING DCM3D

5.1 Required Data

5.1.1 Non-hydrologic Data 45

5.1.2 Hydrologic Data 46

5.2 Data Input for an Initial Run 47

5.3 Data Input for a Restart Run 77

5.3.1 Input File Modifications to Group A $\quad 77$

5.3.2 Input File Modification to Group D 78

5.3.3 Other Modifications to Input Files 78 
5.4 Dimensioning Arrays $\quad 78$

$\begin{array}{ll}5.4 .1 \mathrm{MAXBC} & 79\end{array}$

5.4.2 MAXDX $\quad 79$

$\begin{array}{ll}5.4 .3 \text { MAXDY } & 79\end{array}$

$\begin{array}{ll}5.4 .4 \mathrm{MAXDZ} & 79\end{array}$

5.4.5 MAXLRW $\quad 79$

5.4.6 MAXMAT $\quad 79$

5.4.7 MAXSRC 80

5.4.8 MAXTIM : 80

5.4.9 MAXWRT $\quad 80$

5.5 Running with Head 80

5.6 Plot File Structure 81

5.7 Compiling, Linking, and Running DCM3D 82

$\begin{array}{lll}6.0 & \text { ILLUSTRATIVE PROBLEMS } & 87\end{array}$

6.1 One-Dimensional Horizontal Infiltration 87

6.2 One-Dimensional Horizontal Saturated Matrix/Fracture Flow 91

6.3 Well Flow in a Fractured Porous Media 91

6.4 Two-Dimensional Recharge 99

7.0 REFERENCES $\quad 107$

APPENDIX A: One-Dimensional, Saturated, Fractured, Porous Medium
Analytical Solution.

APPENDIX B: Input Data for the Well Flow in a Fractured.
Porous Medium Problem.

APPENDIX C: Input Data for the Two-Dimensional Recharge Problem 126 
3-1 Grid Notation for Finite Differencing 18

4-1 Flow Chart for DCM3D Main Driver 30

4-2 Flow Chart for DCM3D Moisture Content/Saturation Calculations 39

4-3 Flow Chart for DCM3D Darcy Velocity Calculations 40

4-4 Flow Chart for Subroutine DYDTEQ $\quad 42$

4-5 Coefficient Evaluation Logic for Subroutine DYDTEQ 43

5-1 Example of Gravity Description 50

5-2 Flow Chart for Selecting Boundary Conditions 62

5-3 Flow Chart for Reading Boundary Conditions 65

5-4 Flow Chart for Selecting Source Terms $\quad 74$

5-5 Flow Chart for Reading Source Terms 76

6-1 Schematic of the One-Dimensional Horizontal Infiltration Problem 88

6-2 Characteristic Curves for the One-Dimensional Horizontal

Infiltration Problem

6-3 DCM3D Results for the One-Dimensional Horizontal

Infiltration Problem

6-4 Schematic of the One-Dimensional Horizontal Saturated Matrix/Fracture Flow Problem

6-5 Fracture Head Results for the One-Dimensional Horizontal Saturated Matrix/Fracture Flow Problem

6-6 Porous Medium Head Results for the One-Dimensional Horizontal

6-7 Schematic of Well Flow in a Fractured Porous Medium 100

6-8 DCM3D Results for Wel1 Flow in a Fractured Porous Medium 101

6-9 Schematic of Two-Dimensional Recharge Problem 103

6-10 Characteristic Curves for the Two-Dimensional Recharge Problem 104

6-11 DCM3D Results for Two-Dimensional Recharge Problem 106 
Table

Page

5-1 FORTRAN Program READPL

83

6-1 Input Data for the Horizontal Infiltration Problem

6-2 Characteristic Curve Function Subprograms for the Horizontal Infiltration Problem

6-3 Input Data for the One-Dimensional Horizontal Saturated Matrix/Fracture Flow Problem

6-4 Hydraulic Conductivity Function Subprogram for Two-Dimensional Recharge Problem 
Several people were responsible for helping with this report. At Sandia National Laboratories, Kenneth E. Washington tested the LSODES ordinary differential equation solver on the SNL Cray computer; James T. McCord and Roger R. Eaton reviewed the report and provided many useful comments; Philip I. Pohl helped with several of the figures; and David P. Gallegos served as the SNL project monitor for the DCM3D development. Timothy J. McCartin and John D. Randall served as the technical monitors for the Nuclear Regulatory Commission. 


\subsection{INTRODUCTION}

\subsection{Overview}

Unsaturated, fractured tuff is presently being considered as a potential host rock for the disposal of high-level radioactive waste (HLW) in the United States. The U.S. Department of Energy (DOE) is considering the welded tuffs at Yucca Mountain, Nevada, for HLW disposa1. Under the Nuclear Waste Policy Act of 1982, the Nuclear Regulatory Commission (NRC) is responsible for the regulation of DOE's HLW disposal activities. NRC is developing a performance assessment methodology for analyzing the long-term disposal of HLW in welded tuff and will use the methodology to conduct independent evaluations of license applications from the DOE to construct a HLW repository. NRC has contracted to Sandia National Laboratories (SNL) to assist in the development of this methodology. One of the steps in this work is to develop a computer code to model isothermal ground-water flow in an unsaturated, fractured, porous medium.

\subsection{Background and Objectives}

The first step in the ground-water flow code development process was to identify important ground-water flow processes in the unsaturated, fractured zone around a HLW repository. After determining these processes, a rigorous study was conducted to identify, screen, and test several existing computer codes to determine if any were adequate for modeling unsaturated ground-water flow around a HLW repository (Updegraff, 1989). Updegraff found that the reviewed computer codes could not explicitly handle fractured, porous media. It is important for a code to model fracture flow because fractures exist around potential repository sites. In addition, the reviewed computer codes had some difficulty with handling different types of boundary conditions, and with time step sizes. These difficulties existed even for relatively simple problems. Based on the results of this study, the decision was made to use a code whose capabilities resembled the problem being simulated.

It was decided to use existing ground-water flow codes to simulate flow in the saturated zone, to use an existing code to simulate coupled groundwater flow and heat transfer near the repository, and to develop a new code to simulate isothermal flow in an unsaturated, fractured porous medium in the far field. This report presents the development of the theory and the computer code, DCM3D, for modeling isothermal ground-water flow in an unsaturated, fractured, porous medium.

\subsection{Purpose and Need}

The purpose behind the development of an independent computer code is to provide the NRC with the ability to predict ground-water travel times and velocities for unsaturated, fractured rock. In doing so, this code should possess the ability to predict both porous media and fracture velocity fields, pressures, and moisture contents to be used by the radionuclide transport code which is also being developed as a part of this methodology. 
In addition, the code must easily handle several types of boundary conditions. Because the code is to be used as part of a performance assessment methodology, it must be efficient in performing the calculations.

It has been concluded, based on the investigative studies mentioned above, that a new ground-water flow code is needed to efficiently simulate isothermal ground-water flow in an unsaturated, fractured porous medium. This computer code would provide the NRC with an independent mechanism to evaluate compliance with the numerical criteria set forth in $10 \mathrm{CFR}$ Part 60 and 40 CFR Part 191.

\subsection{Organization}

This report consists of seven sections. They provide the underlying theory, numerical implementation, and data input guide for the new flow code, DCM3D. Section 2 presents the underlying theory used to develop the flow model. This chapter discusses the physical processes, governing equations, and assumptions used to develop the flow model. Section 3 describes the numerical algorithms used to solve the governing equations. Section 4 describes the code structure. Flow charts are provided for the overall code driver and the main subroutines. Section 5 lists the code data requirements. This chapter serves as the data input guide and provides general instructions for running DCM3D. Section 6 presents some illustrative problems solved by DCM3D and compares the results with analytical solutions or experimental results. 


\subsection{Background}

No computer code has modeled ground-water flow in an unsaturated, fractured, porous medium a with a dual continuum approach. Some flow codes can model flow in a fractured, porous medium by treating fractures discretely: Pruess (1987) modeled a heat induced flow in a fractured, porous medium. Pruess' work involved treating the fractures discretely. This is done by putting a part of the grid in the fractures and assigning the fractures their own hydrologic properties. TRACR3D is another computer code that solves for discrete fracture flow (Travis, 1984). The problem with the discrete approach is that, if there are many fractures, the flow field becomes cumbersome to solve for.

Klavetter and Peters (1986a) proposed an equivalent porous medium model for ground-water flow in a fractured, porous medium. Their model starts as a dual continuum model where the porous medium represents one continuum and the fractures another. The flows in each continuum are coupled by a fracture-porous medium transfer term of the form:

$$
\Gamma=\gamma\left(p^{m}-p^{f}\right)
$$

where

$\begin{array}{ll}\Gamma & =\text { volumetric transfer term per unit volume of continuum } \\ \gamma & =\text { transfer coefficient } \\ p^{m} & =\text { water pressure in the porous medium continuum } \\ p^{\mathrm{f}} & =\text { water pressure in the fracture continuum. }\end{array}$

This transfer term is used in hydrogeology and the petroleum industry (Streltsova-Adams, 1978; van Golf-Racht, 1982).

Klavetter and Peters (1986a) then assume that the pressure in the fractures is the same as that in the porous medium. Their assumption is based on the modeling work of Travis et al. (1984), Wang and Narasimhan (1986), and Martinez (1988), and the belief that recharge at Yucca Mountain is on the order of $0.5 \mathrm{~mm} / \mathrm{yr}$ ( $\mathrm{DOE}, 1986$ ). While not indicated in their report, this assumption is equivalent to stating that the transfer coefficient between the porous medium and fracture pressures in Eq. 2-1 is infinitely large. With an infinitely large transfer coefficient, even small pressure differences can cause the transmission of large amounts of water between the fractures and porous medium.

For saturated ground-water flow, the transfer coefficient between the porous medium and the fractures depends on the hydraulic conductivity of the porous medium (Streltsova-Adams, 1978).. If this concept is extended to unsaturated flow then the transfer coefficient would depend on the degree of saturation of the porous medium. The degree of saturation ultimately affects the value of the unsaturated hydraulic conductivity. If the degree of saturation in the porous medium becomes small, the transfer coefficient 
also becomes smal1. In this case the water transfer between the porous medium and the fractures becomes restricted.

The mathematical model and corresponding computer code, DCM3D, developed in this report, attempt to resolve some of the limitations described in the preceding paragraphs. The model developed uses a dual continuum approach similar to Klavetter and Peters (1986a). With this approach tracking flow in each fracture is not done as in the discrete approach. Instead, average flows in a fracture continuum and a porous medium continuum are modeled. In addition, the model also includes a relative permeability term in the porous medium to fracture transfer coefficient. This helps to restrict some of the transfer of water between the porous medium and the fractures when the porous medium becomes dry.

\subsection{Assumptions}

Several assumptions are made regarding flow in an unsaturated, fractured, porous medium. These include the concept of double continuum models, Darcy's law extension to unsaturated flow, and the use of the van Genuchten characteristic curves. There are several other less important assumptions regarding water, fracture, and porous medium compressibilities.

\subsubsection{Double Continuum Mode1}

In general, flow in a porous medium occurs through a complex network of microscopic pores. It is impossible to model the flow in all the separate pores. As such, the flow equations are generally formulated in an average sense over a volume known as a representative elementary volume or REV. This type of formulation is known as a continuum approach (Bear, 1979). Over this REV, average values of the hydrologic properties can be obtained.

The REV is not a fixed size. The size of the REV is subject to two restrictions. The first restriction is that the REV must be large enough that microscopic effects of the water and soil can be meaningfully averaged over the volume. If the REV volume is so small that it falls entirely inside a pore, only the effects of water in the pore would be included in the flow equation. The friction between the soil grain and the water would not be included. If an REV volume falls entirely within a soil grain, no water characteristics would enter into the flow equation or the flow equation would not exist for that particular point. Therefore, the minimum REV volume must enclose many pores or grains. The second restriction is that the volume of the REV must be small enough such that the properties of the porous medium are approximately homogeneous within the REV. It must be small enough so that regional changes in porosity or hydraulic conductivity are outside the volume of an REV. Bear (1972, 1979) and Bear and Bachmat (1990) describe the REV concept and REV averaging theorems in more detail.

Double continuum models consist of two interconnected continua. For the model developed here, one continuum is the porous medium and the other continuum is the fractures. The continua are connected because flow is allowed to occur between them by means of a transfer term. 
A problem which may arise in the dual continuum approach occurs when there is a significant difference in scales between pores and the fractures. In general, the fracture scale is much larger than the pore scale. In a porous medium flow occurs only through the pore space. Klavetter and Peters (1986b) present pore size distributions for the tuffs at Yucca Mountain. The data indicate that pore sizes have a mean of approximately 0.1 microns with most pore sizes less than 1.0 micron. In fractures, flow occurs in the aperture or region between fracture walls.. Wang and Narasimhan (1986) calculate fracture apertures in the 100-200 micron range for Yucca Mountain tuffs. Klavetter and Peters summarize fracture aperture data in the 6 micron to 27 micron range. Thus, it can be determined that the scale difference for the flow areas between tuff fractures and tuff pores is greater than 60 and can be greater than 2000 .

The scale difference for the distance between the flow area in a fracture and the flow area in a porous medium is large also. For a fractured medium the distance between flow areas is the fracture spacing. Wang and Narasimhan (1986) present fracture spacing data for Yucca Mountain tuffs in the $0.10 \mathrm{~m}$ to $0.25 \mathrm{~m}$ range. For soils the distance between flow areas is the grain size. Grain sizes of soils are generally less than 10.0 microns (Bear, 1972). Therefore, the scale difference for the distance between flow areas is 10000 to 25000, which is approximately 10 to 100 times larger than the flow area scale difference.

If the scales of the pores and the fracture are nearly equivalent, then a double porosity model is applicable. If the fracture scale is much larger than the pore scale, then the double porosity approach is probably not valid and a discrete fracture approach may be more applicable.

In a porous medium the minimum REV volume may cover several pore sizes or grain sizes, say a cube with sides whose length is on the order of $0.01 \mathrm{~m}$. Then the REV volume for the fractures would be a cube with sides whose length is $100 \mathrm{~m}$ on a side. If the regional effects in the pore properties do not change over this $100 \mathrm{~m}$ distance then the REV sizes of the pores and the fractures are compatible and a double continuum model is appropriate to model flow in the fractured porous medium. If the regional effects do change over this distance, then a discrete model is probably more appropriate to model ground-water flow. Bear and Bachmat (1990) provide some insight into the scale difference between pores and fractures for a fractured porous medium. This report assumes that the scales of the fractures and pores are nearly equivalent and that a double continuum model can be used to model ground-water flow.. Such a situation would occur in a highly fractured system caused by cooling of welded tuffs.

\subsubsection{Darcy's Law}

Darcy's law is generally used as the governing motion equation for saturated flow (Bear, 1979). It is also assumed that a modified form of Darcy's law applies as the motion equation for unsaturated flow (Bear, 1979). Darcy's law for unsaturated flow has the form: 


$$
\vec{q}^{\omega}=-\frac{\bar{k}_{s}^{\omega} k_{r}}{\mu}\left(\nabla p^{\omega}+\rho g \nabla z\right)
$$

where

$\begin{array}{ll}\overrightarrow{\underline{g}}^{\omega} & =\text { Darcy's velocity vector } \\ \vec{k}_{\mathrm{s}}^{\omega} & =\text { the saturated intrinsic permeability tensor } \\ k_{\mathrm{r}}^{\omega} & =\text { relative permeability } \\ p^{\omega} & =\text { water pressure } \\ \mu & =\text { dynamic water viscosity } \\ \rho & =\text { water density } \\ g & =\text { gravitational constant } \\ z & =\text { vertical distance. }\end{array}$

Throughout this report the " $\omega "$ superscript indicates a fracture property if it is set to " $f$ ", and a porous medium property if it is set to " $m$ ". It is assumed that Eq. 2-2 is valid both for the porous medium continuum and the fracture continuum.

The relative intrinsic permeability depends on the degree of saturation or capillary pressure. It tends to decrease as the saturation decreases or capillary pressure increases. As the saturation of water decreases, the water becomes discontinuous in the pore space. Flow can no longer take place through the pores where the water is discontinuous. Typically the larger pores drain before and fill after the smaller ones. Since only pores containing a continuous phase of water can transmit water, the relative intrinsic permeability must decrease as the saturation decreases. The relative intrinsic permeability has a range between 0.0 for totally dry soils and 1.0 for saturated soils.

\subsubsection{Van Genuchten Characteristic Curves}

Several functional forms exist for describing the relationship between the degree of saturation and the relative intrinsic permeability. Many of these are described in Bear (1979). For the model described here, the van Genuchten characteristic curves are chosen to represent the saturation and relative permeability characteristic curves (van Genuchten, 1980). These equations describe the characteristic curves very well in many cases (Klavetter and Peters, 1986a).

Van Genuchten's equations are an extension of the equations based on Mualem's method (Mualem, 1976). For a description of this extension, refer to van Genuchten (1980). There are two van Genuchten equations. The first one describes the relationship between the capillary pressure and the degree of saturation: 


$$
S_{e}^{\omega}=\left[\frac{1}{1+\left(\alpha^{\omega} p_{c}^{\omega}\right)^{\nu}}\right]^{\lambda^{\omega}}
$$

where

$$
\begin{aligned}
S_{e}^{\omega} & =\text { effective degree of saturation } \\
p_{c}^{\omega} & =\text { capillary pressure } \\
\nu^{\omega} & =\text { parameter } \\
\lambda^{\omega} & =\text { parameter }\left(1-1 / \nu^{\omega}\right) \\
\alpha^{\omega} & =\text { parameter. }
\end{aligned}
$$

The second equation describes the relationship between the relative permeability and the degree of saturation:

$$
k_{r}^{\omega}=\sqrt{S_{e}^{\omega}}\left(1-\left(1-\left(S_{e}^{\omega}\right)^{1 / \lambda^{\omega}}\right)^{\lambda^{\omega}}\right)^{2}
$$

The effective degree of saturation is:

$$
S_{\theta}^{\omega}=\frac{S^{\omega}-S_{\mathbf{r}}^{\omega}}{S_{\mathbf{s}}^{\omega}-S_{\mathbf{r}}^{\omega}}
$$

where

$$
\begin{aligned}
& S^{\omega}=\text { degree of saturation } \\
& S_{\mathbf{r}}^{\omega}=\text { residual degree of saturation } \\
& S_{\mathbf{s}}^{\omega}=\text { saturated degree of saturation (usually } 1.0 \text { ). }
\end{aligned}
$$

\subsubsection{Other Assumptions}

The spatial changes in the water density are assumed to be much smaller than the temporal changes. Under this assumption," spatial changes in the water density can be neglected in the equation formulation.

Water is also assumed to be slightly compressible. The compressibility for a slightly compressible fluid is (Bear, 1972):

$$
\beta=\frac{1}{\rho} \frac{\partial \rho}{\partial p^{\omega}}
$$

where

$$
\begin{array}{ll}
\beta & =\text { compressibility of water } \\
\rho & =\text { density of water }
\end{array}
$$




$$
p^{\omega} \quad=\quad \text { water pressure. }
$$

In addition, it is assumed that both the fracture and the porous medium are slightly elastic. The deformation of the fracture and the porous medium is then governed by (Bear, 1972):

$$
\alpha_{b}^{\omega}=\frac{1}{1-n^{\omega}} \frac{\partial n^{\omega}}{\partial p^{\omega}}
$$

where

$$
\begin{aligned}
& \alpha_{b}^{\omega} \quad=\text { bulk compressibility of the continuum } \\
& n^{\omega} \quad=\text { porosity of the continuum. }
\end{aligned}
$$

\subsection{Flow Equations}

Van Golf-Racht (1982) reviews several dual continuum models of liquid flow in a fractured porous medium. These include both single and multiple phase models. The general dual continuum equations of water flux in an unsaturated, fractured, porous medium can be written as:

$$
\frac{\partial}{\partial t}\left(\rho n^{f} S^{f}\right)=-\nabla \cdot\left(\rho \vec{q}^{f}\right)+\rho \Gamma+\rho Q^{f}
$$

for the fracture continuum, and

$$
\frac{\partial}{\partial t}\left(\rho n^{\mathrm{m}} S^{\mathrm{m}}\right)=-\nabla \cdot\left(\rho \vec{q}^{\mathrm{m}}\right)-\rho \Gamma+\rho Q^{\mathrm{m}}
$$

for the porous medium continuum, where

$$
\begin{aligned}
& Q^{\mathrm{m}} \quad=\quad \begin{array}{l}
\text { porous medium volumetric source term per unit volume of } \\
\text { continua }
\end{array} \\
& Q^{\mathrm{f}} \quad=\quad \begin{array}{l}
\text { fracture volumetric source term per unit volume of } \\
\text { continua } \\
\text { volumetric transfer term from porous medium continuum to }
\end{array} \\
& \quad=\quad \begin{array}{l}
\text { the fracture continuum per unit volume of continua } \\
t \quad=\quad \text { time. }
\end{array}
\end{aligned}
$$

For the fracture continuum equation the unsteady term in the Eq. 2-8 can be expanded to yield:

$$
\frac{\partial}{\partial t}\left(\rho n^{f} S^{f}\right)=n^{f} S^{f} \frac{\partial \rho}{\partial t}+\rho S^{f} \frac{\partial n^{f}}{\partial t}+\rho n^{f} \frac{\partial S^{f}}{\partial t}
$$


After application of the chain rule to Eq. 2-10, the unsteady term becomes:

$$
\frac{\partial}{\partial t}\left(\rho n^{f} S^{f}\right)=\left(n^{f} S^{f} \frac{\partial \rho}{\partial p^{f}}+\rho S^{f} \frac{\partial n^{f}}{\partial p^{f}}+\rho n^{f} \frac{\partial S^{f}}{\partial p^{f}}\right) \frac{\partial p^{f}}{\partial t}
$$

After substitution of the fracture and water compressibility relationships, (Eq. 2-6 and Eq. 2-7 from Section 2.2.4), Eq. 2-11 becomes:

$$
\frac{\partial}{\partial t}\left(\rho n^{f} S^{f}\right)=\left(n^{f} S^{f} \beta+S^{f} \alpha_{b}^{f}\left(1-n^{f}\right)+n^{f} \frac{\partial S^{f}}{\partial p^{f}}\right) \rho \frac{\partial p^{f}}{\partial t}
$$

A similar process produces

$$
\frac{\partial}{\partial t}\left(\rho n^{\mathrm{m}} S^{\mathrm{m}}\right)=\left(n^{\mathrm{m}} S^{\mathrm{m}} \beta+S^{\mathrm{m}} \alpha_{\mathrm{b}}^{\mathrm{m}}\left(1-n^{\mathrm{m}}\right)+n^{\mathrm{m}} \frac{\partial S^{\mathrm{m}}}{\partial p^{\mathrm{m}}}\right) \rho \frac{\partial p^{\mathrm{m}}}{\partial t}
$$

for the unsteady term for the porous medium continuum equation.

The expanded unsteady terms (Eq. 2-12 and Eq. 2-13) and Darcy's law (Eq. 2-2) can be substituted into the fracture and porous medium continua equations (Eq. 2-8 and Eq. 2-9). After dividing through by the water density the following governing ground-water flow equations are produced:

$$
\begin{gathered}
\left(n^{\mathrm{f}} S^{\mathrm{f}} \beta+S^{\mathrm{f}} \alpha_{\mathrm{b}}^{\mathrm{f}}\left(1-n^{\mathrm{f}}\right)+n^{\mathrm{f}} \frac{\partial S^{\mathrm{f}}}{\partial p^{\mathrm{f}}}\right) \frac{\partial p^{\mathrm{f}}}{\partial t}= \\
\nabla \cdot\left(\frac{\bar{k}_{\mathrm{s}}^{\mathrm{f}} k_{\mathrm{r}}^{\mathrm{f}}}{\mu}\left(\nabla p^{\mathrm{f}}+\rho g \nabla z\right)\right)+\Gamma+Q^{\mathrm{f}}
\end{gathered}
$$

and

$$
\begin{gathered}
\left(n^{\mathrm{m}} S^{\mathrm{m}} \beta+S^{\mathrm{m}} \alpha_{\mathrm{b}}^{\mathrm{m}}\left(1-n^{\mathrm{m}}\right)+n^{\mathrm{m}} \frac{\partial S^{\mathrm{m}}}{\partial p^{\mathrm{m}}}\right) \frac{\partial p^{\mathrm{m}}}{\partial t}= \\
\nabla \cdot\left(\frac{\bar{k}_{\mathrm{s}}^{\mathrm{m}} k_{\mathrm{r}}^{\mathrm{m}}}{\mu}\left(\nabla p^{\mathrm{m}}+\rho g \nabla z\right)\right)-\Gamma+Q^{\mathrm{m}}
\end{gathered}
$$

Only the volumetric transfer term between the porous medium continuum and the fracture continuum remains to be defined. 


\subsection{Transfer Term}

The transfer term determines the rate of water exchange between the porous medium and the fracture continua. In saturated flow, this transfer term produces a relationship similar to Newton's law of cooling (Bird et al., 1960):

$$
\Gamma=\gamma\left(p^{\mathrm{m}}-p^{\mathrm{f}}\right)
$$

where

$$
\gamma=\begin{aligned}
& \text { porous medium continuum to fracture continuum transfer } \\
& \text { coefficient. }
\end{aligned}
$$

Several authors assume that the transfer coefficient depends on the saturated intrinsic permeability of the porous medium, $k_{\mathrm{s}}^{\mathrm{m}}$, a length parameter, 1, the specific area of the fracture surface per unit volume, $A_{\mathrm{s}}$, and the dynamic viscosity, $\mu$, (Streltsova-Adams, 1978; van Golf-Racht, 1982):

$$
\gamma=A_{\mathrm{s}} \frac{k^{\mathrm{m}}}{\mu I}
$$

However, in the unsaturated zone the intrinsic permeability depends on the degree of saturation of the porous medium. Therefore, it is necessary to modify the transfer coefficient to account for this dependence. This produces:

$$
\gamma=A_{\mathrm{s}} \frac{k_{\mathrm{s}}^{\mathrm{m}} k_{\mathrm{r}}^{\mathrm{m}}}{\mu I}
$$

In the model implemented in this report, the relative permeability is governed by the van Genuchten relative permeability characteristic curve equation (Eq. 2-4).

In order to simplify the transfer term, the saturated intrinsic permeability, $k_{\mathrm{s}}^{\mathrm{m}}$, the fracture specific surface, $A_{\mathrm{s}}$, and the length parameter, 1 , are combined into one dimensionless term, which is called the transfer factor, $A$. The relationship:

$$
A=A_{\mathrm{s}} \frac{k_{\mathrm{s}}^{\mathrm{m}}}{I}
$$


is obtained. The complete porous medium to fracture transfer term is described by:

$$
\Gamma=\frac{A k_{x}^{\mathrm{m}}}{\mu}\left(p^{\mathrm{m}}-p^{\mathrm{f}}\right)
$$

Eq. 2-14, Eq. 2-15, and Eq. 2-20 form the set of equations for saturated/ unsaturated ground-water flow in a fractured, porous medium. 


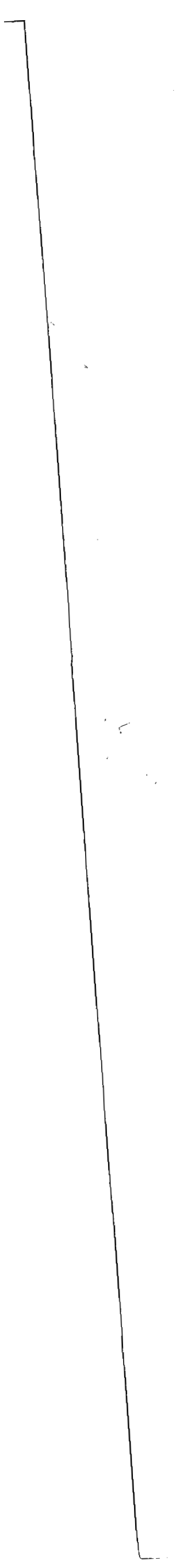




\subsection{NUMERICAL IMPLEMENTATION}

In this section the governing partial differential equations for unsteady, ground-water flow in a variably saturated, fractured, porous medium are summarized. The spatial discretization technique for the equations is then described. The resulting set of time dependent ordinary differential equations are then solved by a general ordinary differential equation solver designed for stiff sets of equations.

\subsection{Flow Mode1 Difference Equations Development}

The ground-water flow equations for the porous medium continuum and fracture continuum, respectively, are:

$$
C^{\mathrm{m}} \frac{\partial p^{\mathrm{m}}}{\partial t}=\nabla \cdot\left(\bar{K}^{\mathrm{m}}\left(\nabla p^{\mathrm{m}}+\rho g \nabla z\right)\right)-\Gamma+Q^{\mathrm{m}}
$$

and

$$
C^{\mathrm{f}} \frac{\partial p^{\mathrm{f}}}{\partial t}=\nabla \cdot\left(\bar{K}^{\mathrm{f}}\left(\nabla p^{\mathrm{f}}+\rho g \nabla z\right)\right)+\Gamma+Q^{\mathrm{f}}
$$

where

$$
\begin{aligned}
& p^{\mathrm{m}} \quad=\quad \text { water pressure in the porous medium continuum } \\
& p^{f}=\text { water pressure in the fracture continuum } \\
& C^{\mathrm{m}}=\text { specific storage coefficient of the porous medium } \\
& \text { continuum } \\
& C^{f}=\text { specific storage coefficient of the fracture continuum } \\
& \bar{K}^{\mathrm{m}}=\text { mobility coefficient for the porous medium continuum } \\
& \bar{K}^{f} \quad=\quad \text { mobility coefficient for the fracture continuum } \\
& Q^{m} \quad=\quad \text { volumetric porous medium continuum source term per unit } \\
& \text { volume of continua } \\
& Q^{f}=\text { volumetric fracture continuum source term per unit volume } \\
& \text { of continua } \\
& \Gamma=\text { porous medium continuum to fracture continuum transfer } \\
& \text { term } \\
& \begin{array}{ll}
\rho & =\text { water density } \\
g & =
\end{array} \\
& z=z \text {-direction distance } \\
& t=\text { time. }
\end{aligned}
$$

The porous medium and fracture continua mobility coefficients are related to the intrinsic permeabilities as follows: 


$$
\bar{K}^{\mathrm{m}}=\frac{\bar{k}_{\mathrm{s}}^{\mathrm{m}} k_{x}^{\mathrm{m}}}{\mu}
$$

and

$$
\bar{K}^{f}=\frac{\overline{k_{s}^{f}} k_{r}^{f}}{\mu}
$$

where

$$
\begin{aligned}
& \bar{k}_{\mathrm{s}}^{\mathrm{m}}=\begin{array}{l}
\text { saturated intrinsic permeability of the porous medium } \\
\text { continuum }
\end{array} \\
& \bar{k}_{\mathrm{s}}^{\mathrm{f}}=\begin{array}{l}
\text { saturated intrinsic permeability of the fracture } \\
\text { continuum }
\end{array} \\
& k_{\mathrm{r}}^{\mathrm{m}}=\text { relative permeability of the porous medium continuum } \\
& k_{\mathrm{r}}^{\mathrm{f}}=\text { relative permeability of the fracture continuum } \\
& \mu \quad=\text { dynamic viscosity of water }
\end{aligned}
$$

The porous medium and fracture continua relative permeabilities are defined by the van Genuchten relationships (Eq. 2-3 and Eq. 2-4) and, therefore, depend on the porous medium and fracture continua water pressures, respectively. The porous medium and fracture continua specific storage coefficients are defined, respectively, as:

$$
C^{\mathrm{m}}=n^{\mathrm{m}} S^{\mathrm{m}} \beta+S^{\mathrm{m}} \alpha_{\mathrm{b}}^{\mathrm{m}}\left(1-n^{\mathrm{m}}\right)+n^{\mathrm{m}} \frac{\partial S^{\mathrm{m}}}{\partial p^{\mathrm{m}}}
$$

and

$$
C^{\mathrm{f}}=n^{\mathrm{f}} S^{\mathrm{f}} \beta+S^{\mathrm{f}} \alpha_{\mathrm{b}}^{\mathrm{f}}\left(1-n^{\mathrm{f}}\right)+n^{\mathrm{f}} \frac{\partial S^{\mathrm{f}}}{\partial p^{\mathrm{f}}}
$$

where

$$
\begin{array}{ll}
S^{\mathrm{m}} & =\text { porous medium continuum degree of saturation } \\
S^{\mathrm{f}} & =\text { fracture continuum degree of saturation } \\
n^{\mathrm{n}} & =\text { porous medium continuum porosity } \\
n^{\mathrm{f}} & =\text { fracture continuum porosity } \\
\alpha_{\mathrm{b}}^{\mathrm{m}} & =\text { porous medium continuum compressibility } \\
\alpha_{\mathrm{b}}^{\mathrm{f}} & =\text { fracture continuum compressibility } \\
\beta & =\text { water compressibility }
\end{array}
$$

The combined porosity-derivative terms in Eq. 3-5 and Eq. 3-6 are known as the porous medium and fracture specific moisture capacities, respectively. 
Because they depend on the saturation of the porous medium or fracture continua saturations, the specific moisture capacities depend on the porous medium and fracture continua pressures as well.

Finally, the exchange of water is modeled as:

$$
\Gamma=\gamma\left(p^{m}-p^{f}\right)
$$

where

$$
\begin{aligned}
& \gamma=\text { the porous medium continuum to fracture continuum } \\
& \text { transfer coefficient. }
\end{aligned}
$$

The transfer coefficient is described in Section 2.4. Because the transfer coefficient depends on the relative permeability of the porous medium, it also depends on the porous medium water pressure.

A few comments about the degree of saturation and the relative permeabilities are in order. The degree of saturation and relative permeability, are represented by analytical functions represented by the van Genuchten functions (Eq. 2-3 and Eq. 2-4). The derivatives of these functions with respect to pressure are analytical functions also. The models and their derivatives are provided as function subprograms in DCM3D. Alternative degree of saturation and relative permeability models to the van Genuchten ones can be implemented by modifying those function subprograms.

It should be noted that porous medium and fracture specific storage coefficients, $C^{\mathrm{m}}$ and $C^{\mathrm{f}}$, defined by Eq. 3-5 and Eq. 3-6, involve the saturation, which depends on the water pressure. Physically, the specific storage coefficients are greater than or equal to zero for all values of water pressures for both saturated and unsaturated flow and physically reasonable values of the water, porous medium, and fracture compressibilities, and porous medium and fracture porosities. Certain analytic properties must be exhibited by the specific storage coefficients and the relative permeabilities. Let the $\omega$ superscript equal " $m$ " or " $f$ " for porous medium or fracture, respectively. Then, for saturated flow:

$$
p^{\omega} \geq 0, \quad S^{\omega}=1, \quad \frac{\partial S^{\omega}}{\partial p^{\omega}}=0, \quad k_{r}^{\omega}=1
$$

Hence,

$$
C^{\omega}=n^{\omega} \beta+\alpha_{b}^{\omega}\left(1-n^{\omega}\right)>0
$$

For unsaturated flow: 


$$
p^{\omega} \leq 0, \quad 0 \leq S^{\omega} \leq 1, \quad \frac{\partial S^{\omega}}{\partial p^{\omega}} \geq 0, \quad 0 \leq k_{x}^{\omega} \leq 1
$$

Hence,

$$
C^{\omega} \geq n^{\omega} \frac{\partial S^{\omega}}{\partial p^{\omega}} \geq 0
$$

The only time that the specific storage coefficient can approach zero is when the water pressure approaches negative infinity. Maintaining the analytical saturation relationships and the above implied inequalities is important, both for obtaining realistic computational results and in avoiding oscillations in the numerical solutions.

The volume source terms, $Q^{\mathrm{m}}$ and $Q^{\mathrm{f}}$, are allowed to vary linearly in time in each cell volume. The surface pressure or flux boundary conditions are allowed to vary in time and space at each outside boundary surface.

\subsection{Finite Difference Equations}

The time dependent coupled nonlinear ground-water flow equations (Eq. 3-1 and Eq. 3-2) are to be solved numerically for the porous medium and fracture pressures subject to initial and boundary conditions. These flow equations are formulated as a coupled set of spatial finite-difference (FD) time- dependent ordinary differential equations (ODE). The time variable is retained as a continuous dependent variable so that the ODE'S can be solved consistently using the ODEPACK (Hindmarsh, 1983) programs. The specific storage coefficients and relative permeabilities may differ by several orders of magnitude in a particular problem. The resultant coupled ODE's may be relatively "stiff" due to the variability of specific storage coefficients, the relative permeabilities, and the transfer terms. This spatial FD model formulation results in a stiff ODE system similar to those studied and solved by Brown and Hindmarsh (1987).

The spatial FD equations are formulated under the assumption of continuity of the pressure and fluxes at the grid block interfaces. The permeability tensor for the porous medium or fracture is assumed to be a diagonal, but not necessarily isotropic, i.e.

$$
\bar{k}_{\mathrm{s}}^{\omega}=\left[\begin{array}{ccc}
k_{\mathrm{xx}}^{\omega} & 0 & 0 \\
0 & k_{y y}^{\omega} & 0 \\
0 & 0 & k_{z z}^{\omega}
\end{array}\right]
$$


A spatial FD 'BOX' scheme is applied in Cartesian geometry $(x, y, z)$ to Eq. $3-1$ and Eq. $3-2$ with the constraints that the flux and pressure are continuous across cell interfaces and that Gauss' theorem,

$$
\int \nabla \cdot J d V=\int J \cdot d A
$$

is satisfied. The cell nodes are defined at the cell centers, as shown in Figure 3-1. The flux and boundary pressures are evaluated at the cell surfaces, consistent with the FD scheme and Gauss' theorem. For the diagonal permeability approximation used here, the flux at any cell surface is perpendicular to the surface and evaluated at the surface center.

The transfer term in Eq. 3-1 and Eq. 3-2 couples the porous medium and fracture equations only in the same cell. The volume source terms each contribute only to specific porous medium and fracture cells and may be piece-wise linear time dependent.

The FD grid notation is illustrated in the Figure 3-1 for one direction, the $z$ direction, only. A similar figure would hold for the other two dimensions by cyclically relabelling $k$ with $i$ and $i$ with $j$. Cell centered values are denoted by integer subscripts and cell surface values by halfinteger subscripts. The index notation for the central position in the cell is $(i, j, k)$. However, in Figure $3-1$ the $i, j$ indices have been suppressed. The single arrows associated with the flux $(q)$ terms indicate the flux flow direction at the surface center (in the other dimensions). The double arrows associated with the external source $(Q)$ and transfer terms $(\Gamma)$ indicate the cell into which contributions are made.

The porous medium or fracture specific storage coefficient terms are obtained by direct evaluation in terms of the cell centered pressures, $p_{1, j, k}$. The function subprogram for the specific storage coefficient requires the specific hydrologic unit data and van Genuchten equation for the degree of saturation and its derivative with respect to water pressure. Since the pressure is evaluated at the cell centered value, $p_{i, j, k}$, for the specific storage coefficients, iteration to obtain $p_{i, j, k}$ is not required.

Physically, the pressure and flux must be continuous at all internal cell boundaries in this model. The FD coefficients are formulated so that these continuity conditions are satisfied exactly by the model. The porous medium or fracture Darcy flux is:

$$
q=-K(\nabla p+\rho g \nabla z)
$$

where the specific porous medium or fracture superscript notation is temporarily dropped for convenience. The continuity of the flux between the $(i, j, k)$ and $(i, j, k+1)$ grid blocks at the $(i, j, k+1 / 2)$ interface, using Eq. $3-9$, requires two simultaneous relationships to be satisfied at that interface. For the $z$-direction ( $k$ index): 


\section{Pressure}
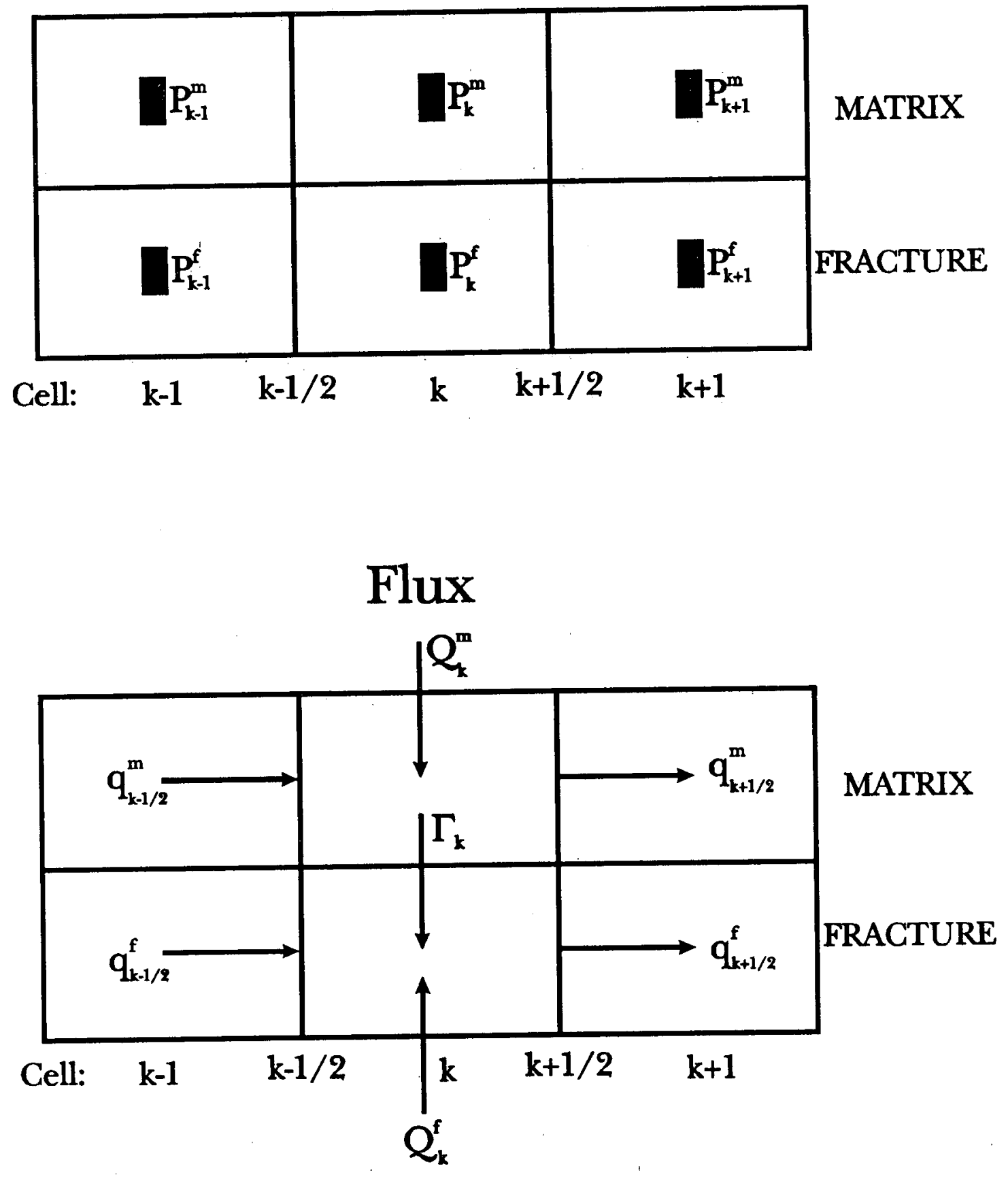

Figure 3-1: Grid Notation for Finite Differencing 


$$
q_{\mathrm{k}+1 / 2}=-K_{\mathrm{k}}^{\mathrm{k}+1 / 2}\left(\frac{p_{\mathrm{k}+1 / 2}-p_{\mathrm{k}}}{\Delta z_{\mathrm{k}} / 2}+\rho g\right)
$$

and

$$
q_{\mathrm{k}+1 / 2}=-K_{\mathrm{k}+1}^{\mathrm{k}+1 / 2}\left(\frac{p_{\mathrm{k}+1}-p_{\mathrm{k}+1 / 2}}{\Delta z_{\mathrm{k}+1} / 2}+\rho g\right)
$$

where

$$
\Delta z_{k}=\text { is the grid block width of the } k^{\text {th }} \text { grid block in the } z \text { - }
$$
direction.

Pressures evaluated at a grid block interface are denoted by the half integer subscript and grid block centered pressures are denoted by integer subscripts. The " $i$ " and " $j$ " indices are suppressed. The grid block spacings may be different for each grid block in each orthogonal direction. The superscript on the mobility coefficient indicates the interface upon which the evaluation is made and the subscript indicates the grid block from which the material properties are determined. Fluxes in the other directions $(x, i ; y, j)$ are similar in form and are not presented here.

If the gravitational acceleration direction is parallel to the $z$-direction only (assumed here), then the terms involving density and gravity are nominally absent from the $\mathrm{x}$-direction and $\mathrm{y}$-direction flux components. Otherwise, the appropriate projected components of density and gravity are used for each mesh direction flux component.

Imposing internal flux continuity and pressure continuity conditions leads to a consistent boundary pressure determination by solving a transcendental relationship. If the grid block centered pressures $p_{\mathrm{k}}$ and $p_{\mathrm{k}+1}$ are continuous functions of time, the continuity of flux and pressure indicates that Eq. 3-10 and Eq. 3-11 are equal. By equating the two equations, the interface pressure, $P_{\mathrm{k}+1 / 2}$, can be determined as:

$$
p_{\mathrm{k}+1 / 2}=\frac{\left(\frac{K_{\mathrm{k}}^{\mathrm{k}+1 / 2}}{\Delta z_{\mathrm{k}}}\right) P_{\mathrm{k}}+\left(\frac{K_{\mathrm{k}+1}^{\mathrm{k}+1 / 2}}{\Delta z_{\mathrm{k}+1}}\right) P_{\mathrm{k}+1}+\frac{\rho g}{2}\left(K_{\mathrm{k}+1}^{\mathrm{k}+1 / 2}-K_{\mathrm{k}}^{\mathrm{k}+1 / 2}\right)}{\frac{K_{\mathrm{k}}^{\mathrm{k}+1 / 2}}{\Delta z_{\mathrm{k}}}+\frac{K_{\mathrm{k}+1}^{\mathrm{k}+1 / 2}}{\Delta z_{\mathrm{k}+1}}}
$$

Substituting this result for $p_{\mathrm{k}+1 / 2}$ into either expression for the flux in Eq. 3-10 or Eq. 3-11 yields: 


$$
q_{k+1 / 2}=-2 \frac{\left(p_{k+1}-p_{k}+\frac{\rho g\left(\Delta z_{k+1}+\Delta z_{k}\right)}{2}\right)}{\frac{\Delta z_{k+1}}{K_{k+1}^{k+1 / 2}}+\frac{\Delta z_{k}}{K_{k}^{k+1 / 2}}}
$$

If the interfacial mobility coefficients, $K_{k}^{k+1 / 2}$, were not also dependent on the boundary pressure, $p_{\mathrm{k}+1 / 2}$, the cell boundary pressure could be solved directly from the flux continuity relationship, Eq. 3-12. However, due to the interfacial mobility coefficient dependence on interface pressure, the interface pressure in Eq. 3-12 must be solved iteratively using the known grid block centered pressures, grid spacings, and material properties of the involved adjacent grid blocks. The interface boundary pressure equation is solved numerically using a combination of bisection, interval halving, and Newton-Raphson iteration, based on the RTSAFE algorithm (Press et a1., 1986). Added safeguards against infinite looping on non-convergent solutions are included. Limiting situations peculiar to this class of problem are recognized. More importantly, the algorithm recognizes if the material types on either side of the interface are the same before implementing an iterative solution. If the material types are the same, then determining $p_{\mathrm{k}+1 / 2}$ in Eq. 3-12 reduces to just a linear interpolation between the cell centered pressures, $p_{k+1}$ and $p_{k}$, and does not require an iterative solution. If $p_{\mathrm{k}}$ and $p_{\mathrm{k}+1}$ are both greater than 0.0 , indicating saturated conditions, and the gravitational terms are nonexistent, then the interface pressure in Eq. 3-12 reduces to a grid block weighted harmonic mean of $p_{\mathrm{k}}$ and $p_{\mathrm{k}+1}$.

ODEPACK frequently evaluates $\partial p_{i, j, k}^{\mathrm{m}} / \partial t$ and $\partial p_{i, j, k}^{\mathrm{f}} / \partial t$, in the FD equations derived below. This involves reconstruction of the FD coefficients on each subroutine call. The strategy of recognizing the same material type on each side of an interface results in significant computational speed improvements in the FD coefficient generation by avoiding iteration when direct interpolation will suffice.

The cell volumes, $V_{i, j, k}$, and cell surface areas, $\left(A_{1}, A_{j}\right.$, and $\left.A_{\mathrm{k}}\right)$ are defined as:

$$
\begin{aligned}
V_{1, j, \mathrm{k}} & =\Delta \mathrm{x}_{\mathrm{i}} \Delta \mathrm{y}_{\mathrm{j}} \Delta z_{\mathrm{k}}, \\
A_{\mathrm{i}} & =\Delta y_{\mathrm{j}} \Delta z_{\mathrm{k}}, \\
A_{\mathrm{j}} & =\Delta \mathrm{x}_{1} \Delta z_{\mathrm{k}}, \\
A_{\mathrm{k}} & =\Delta \mathrm{x}_{\mathrm{i}} \Delta \mathrm{y}_{\mathrm{j}} \\
\text { where } & =\text { the width of the } \mathrm{i}^{\text {th }} \text { grid block in the x direction } \\
\Delta \mathrm{y}_{\mathrm{j}} & =\text { the width of the } \mathrm{j}^{\text {th }} \text { grid block in the y direction. }
\end{aligned}
$$

After integration of the flow equations over the grid block volume and application of Gauss' theorem, the internal node FD time dependent equations (with implicit flux and pressure continuity at interfaces) are: 


$$
\begin{gathered}
V_{1, j, k} C_{i, j, k}^{m}\left(p_{i, j, k}^{m}\right) \frac{\partial p_{i, j, k}^{m}}{\partial t}= \\
a_{i}^{m} p_{i+1, j, k}^{m}-\left(a_{i}^{m}+b_{i}^{m}\right) p_{i, j, k}^{m}+b_{i}^{m} p_{i-1, j, k}+ \\
c_{j}^{m} p_{i, j+1, k}^{m}-\left(c_{j}^{m}+d_{j}^{m}\right) p_{i, j, k}^{m}+d_{j}^{m} p_{i, j-1, k}^{m}+ \\
e_{k}^{m} p_{i, j, k+1}^{m}-\left(e_{k}^{m}+f_{k}^{m}\right) p_{i, j, k}^{m}+f_{k}^{m} p_{i, j, k-1}^{m}- \\
V_{i, j, k} \gamma_{i, j, k}\left(p_{i, j, k}^{m}-p_{i, j, k}^{f}\right)+Q_{i, j, k}^{m}+G_{1, j, k}^{m}
\end{gathered}
$$

and

$$
\begin{gathered}
V_{1, j, k} C_{i, j, k}^{f}\left(p_{i, j, k}^{f}\right) \frac{\partial p_{i, j, k}^{f}}{\partial t}= \\
a_{1}^{f} p_{i+1, j, k}^{f}-\left(a_{1}^{f}+b_{i}^{f}\right) p_{i, j, k}^{f}+b_{i}^{f} p_{i-1, j, k}^{f}+ \\
c_{j}^{f} p_{i, j+1, k}^{f}-\left(c_{j}^{f}+d_{j}^{f}\right) p_{i, j, k}^{f}+d_{j}^{f} p_{i, j-1, k}^{f}+ \\
e_{k}^{f} p_{i, j, k+1}^{f}-\left(e_{k}^{f}+f_{k}^{f}\right) p_{i, j, k}^{f}+f_{k}^{f} p_{i, j, k-1}^{f}+ \\
V_{i, j, k} \gamma_{i, j, k}\left(p_{i, j, k}^{m}-p_{i, j, k}^{f}\right)+Q_{i, j, k}+G_{i, j, k}^{f}
\end{gathered}
$$

where

$$
a_{i}^{\omega}=b_{i+1}^{\omega}, \quad c_{j}^{\omega}=d_{j+1}^{\omega}, \quad e_{k}^{\omega}=f_{k+1}^{\omega}
$$

Thus, the coefficient matrices for the flux terms explicitly involving the pressure are symmetrical. This implies that the eigenspectra for a problem linearized about any solution satisfying the boundary conditions are always real and will not lead to complex oscillatory behavior due to the FD coefficients. The contributions from the flux involving the gravity terms occur either as contributions to the diagonal coefficients (those multiplying the $p_{i, j, k}$ terms) or to the gravitational source term, $G_{i, j, k}$, which also includes contributions from imposed boundary conditions.

The internal cell coefficient terms are:

$$
a_{i}^{\omega}=\frac{2 A_{i}}{\frac{\Delta x_{i+1}}{K_{x x_{i+1, j, k}}^{\omega ; 1+1 / 2}\left(p_{i+1 / 2, j, k}^{\omega}\right)}+\frac{\Delta x_{1}}{K_{x x_{1, j}, \mathrm{k}}^{\omega, 1+1 / 2}\left(p_{i+1 / 2, j, k}^{\omega}\right)}}
$$




$$
c_{j}^{\omega}=\frac{2 A_{j}}{\frac{\Delta y_{j+1}}{K_{y y_{i, j+1, k}}^{\omega, j+1 / 2}\left(p_{i, j+1 / 2, k}^{\omega}\right)}+\frac{\Delta y_{j}}{K_{y y_{i, j, k}}^{\omega, j+1 / 2}\left(p_{i, j+1 / 2, k}^{\omega}\right)}}
$$

and

$$
e_{\mathrm{k}}^{\omega}=\frac{2 A_{\mathrm{k}}}{\frac{\Delta z_{\mathrm{k}+1}}{K_{\mathrm{zz}}^{\omega, \mathrm{k}+1 / 2}\left(p_{i, \mathrm{k}+1}^{\omega}\left(p_{i, j, \mathrm{k}+1 / 2}\right)\right.}+\frac{\Delta z_{\mathrm{k}}}{K_{\mathrm{zz}, \mathrm{k}, \mathrm{k}}^{\omega, 1 / 2}\left(p_{i, j, \mathrm{k}+1 / 2}^{\omega}\right)}}
$$

ODEPACK requires subroutine input that generates $\partial p_{i, j, k}^{\mathrm{m}} / \partial t$ and $\partial p_{i, j, k}^{\mathrm{f}} / \partial t$ from Eqs. 3-15 and 3-16 for each $(i, j, k)$ cell at time, $t$. The coefficient terms, Eq. 3-18, Eq. 3-19 and Eq. 3-20, multiplying specific the cell pressures in Eqs. $3-15$ and 3-16, are evaluated as functions. Since these coefficients involve the cell boundary pressures, the nonlinearity due to the permeability dependence upon cell boundary pressures must be solved simultaneously. The self-consistent boundary pressure is determined uniquely by imposing continuity of flux and pressure at the surface and solving Eq. 3-12. Thus, the calculation of $\partial p_{i, j, k}^{\mathrm{m}} / \partial t$ and $\partial p_{i, j, k}^{\mathrm{f}} / \partial t$ for ODEPACK can be performed efficiently by making the coefficient evaluations of Eq. 3-18, Eq. 3-19, and Eq. 3-20 a function call inside of which the boundary pressure terms are determined self-consistently from Eq. 3-12. Similarly, if the imposed boundary conditions (pressure or flux) are determined as modifications of the pressure coefficients, contributions to $G_{i, j, k}^{\omega}$ and/or $Q_{1, j, k}^{\omega}$ for $\operatorname{specific~}(i, j, k)$ indices, then the implementation of time- and space-dependent boundary conditions is a straightforward programming task.

The $G_{i, j, k}^{\omega}$ gravitational terms for the porous medium and fracture arise only from nonzero " $\rho \mathrm{g}$ " terms in the corresponding flux and from contributions due to the imposed exterior boundary conditions. The $G_{1, j, k}^{\omega}$ contributions for the gravitational acceleration, $g$, parallel to the $z$-axis are:

$$
G_{i, j, k}^{\omega}=A_{\mathrm{k}} \rho g\left(\frac{\Delta z_{\mathrm{k}}+\Delta z_{\mathrm{k}+1}}{\frac{\Delta z_{\mathrm{k}+1}}{K_{\mathrm{i}, \mathrm{j}, \mathrm{k}+1}^{\omega, \mathrm{k}+1 / 2}}+\frac{\Delta z_{\mathrm{k}}}{K_{\mathrm{i}, \mathrm{j}, \mathrm{k}}^{\omega, \mathrm{k}+1 / 2}}}-\frac{\Delta z_{\mathrm{k}}+\Delta z_{\mathrm{k}-1}}{\frac{\Delta z_{\mathrm{k}}}{K_{1, \mathrm{k}}^{\omega, \mathrm{k}-1 / 2}}+\frac{\Delta z_{\mathrm{k}-1}}{K_{\mathrm{i}, \mathrm{j}, \mathrm{k}-1}^{\omega, \mathrm{k}-1 / 2}}}\right)
$$

If the direction of $g$ is not parallel to $z$, the component contributions in Eq. 3-21 in each of the $i, j$, and $k$ directions must be appended to this result cyclically $(k>i>j>k)$, as needed. 
Except for an additional factor involving " $\rho$ " and the adjacent cell spacings, the coefficients in Eqs. 3-22 are the coefficients already determined in Eq. 3-18, Eq. 3-19, and Eq. 3-20.

\subsection{External Boundary Conditions}

The equation modifications required for imposing external pressure or flux boundary conditions only involve modifying the coefficient terms in the balance equations. Since each direction is treated in the same manner for both porous medium and fracture, the method is detailed here only for the porous medium and for a single mesh direction, the $z$ direction. The corresponding implementation for the fracture boundary conditions is obtained by interchanging the superscripts " $m$ " and " $f$ " in the equations below. The implementation of the boundary conditions in the other directions is obtained by cyclically interchanging indices $(k>i>j>k)$, taking into account the possible appending of the $G_{1, j, k}$ terms in the case that $g$ is not parallel to the $z$ direction.

The porous medium balance equation has the form as Eq. 3-15. In terms of the Darcy flux, $q$, the equation can be rewritten as:

$$
\begin{gathered}
V_{i, j, k} C_{i, j, k}^{m}\left(p_{i, j, k}^{m}\right) \frac{\partial p_{i, j, k}^{m}}{\partial t}= \\
A_{i}\left(q_{i-1 / 2, j, k}^{m}-q_{i+1 / 2, j, k}^{m}\right)+ \\
A_{j}\left(q_{i, j-1 / 2, k}^{m}-q_{i, j+1 / 2, k}^{m}\right)+ \\
A_{k}\left(q_{i, j, k-1 / 2}^{m}-q_{i, j, k+1 / 2}^{m}\right)- \\
V_{i, j, k} \gamma_{i, j, k}\left(p_{i, j, k}^{m}-p_{i, j, k}^{f}\right)+V_{1, j, k} Q_{1, j, k}^{m}
\end{gathered}
$$

where the $G_{i, j, k}^{\mathrm{m}}$ contributions of Eq. 3-15 are now contained in the flux terms.

\subsubsection{Flux Boundary Conditions:}

If the porous medium flux $q_{i, j, 1 / 2}^{m}$ is specified (known) at the left most boundary in the $z$ direction, $k=1 / 2$, then the flux,

$$
q_{1, j, 1 / 2}^{\mathrm{m}}=-K_{1, j, 1}^{\mathrm{m}, 1 / 2}\left(\frac{p_{1, j, 1}^{\mathrm{m}}-\dot{p}_{1, \mathrm{j}, 1 / 2}^{\mathrm{m}}}{\Delta z_{1} / 2}+\rho g\right]
$$

is substituted into the flux balance equation, Eq. 3-22, which implies $\mathrm{f}_{\mathrm{k}=1}=0$ and 


$$
G_{1, j, 1}^{\mathrm{m}}=A_{\mathrm{k}=1}\left(q_{1, j, 1 / 2}^{\mathrm{m}}+\frac{\rho g\left(\Delta z_{1}+\Delta z_{2}\right)}{\frac{\Delta z_{1}}{K_{1, j, 1}^{\mathrm{m}, 3 / 2}}+\frac{\Delta z_{2}}{K_{1, j, 2}^{\mathrm{m}, 3 / 2}}}\right)
$$

in the coefficient balance equation, Eq. 3-15.

Similarly, if the flux $q_{i, j, n+1 / 2}^{m}$ is specified (known) at the right boundary in the $z$ direction, $k=n+1 / 2$, then the flux,

$$
q_{i, j, n+1 / 2}^{m}=-K_{i, j, n}^{m}, n+1 / 2\left(\frac{p_{i, j, n+1 / 2}^{m}-p_{i, j, n-1 / 2}^{m}}{\Delta z_{n} / 2}+\rho g\right)
$$

is substituted into the flux balance equation, Eq. 3-22, which implies $e_{\mathrm{k}=\mathrm{n}}=0$ and

$$
G_{1, j, \mathrm{n}}^{\mathrm{m}}=A_{\mathrm{k}=\mathrm{n}}\left(q_{1, \mathrm{j}, \mathrm{n}+1 / 2}^{\mathrm{m}}-\frac{\rho g\left(\Delta z_{\mathrm{n}-1}+\Delta z_{\mathrm{n}}\right)}{\frac{\Delta z_{\mathrm{n}-1}}{K_{1, j, \mathrm{n}-1}^{\mathrm{m}, \mathrm{n}-1 / 2}}+\frac{\Delta z_{\mathrm{n}}}{K_{1, j, \mathrm{n}}^{\mathrm{m}, \mathrm{n}-1 / 2}}}\right)
$$

in the coefficient balance equation, Eq. 3-15. The corresponding flux boundary condition implementation for the fracture equation is obtained by interchanging the superscripts " $m$ " and " $f$ ". The implementation of flux boundary conditions in the other directions is obtained by cyclically interchanging the indices as $k>i>j>k$, taking into account the possible appending of the advection term in the case that gravity is not parallel to the $z$ direction.

\subsubsection{Pressure Boundary Conditions}

If the boundary pressure $p_{i, j, 1 / 2}^{\mathrm{m}}$ is specified (known) at the left most boundary in the $z$ direction, $k=1 / 2$, then the flux,

$$
q_{i, j, 1 / 2}^{\mathrm{m}}=-K_{i, j, 1}^{\mathrm{m}, 1 / 2}\left(\frac{p_{i, j, 1}^{\mathrm{m}}-p_{1, j, 1 / 2}^{\mathrm{m}}}{\Delta z_{1} / 2}+\rho g\right)
$$

is substituted into the flux balance equation, Eq. 3-22, where the boundary pressure is known. This implies, in the porous medium balance equation, Eq. 3-15, that: $f_{\mathrm{k}=1}=0$; the coefficient $e_{\mathrm{k}=1}$ is calculated as for an interior ce11; the coefficient of $p_{i, j, k=1}^{m}$ is modified from $e_{1}+f_{1}$ to $e_{1}+f_{k=1}^{\prime}$, where 


$$
f_{\mathrm{k}=1}^{\mathrm{m}^{\prime}}=\frac{2 A_{\mathrm{k}=1}}{\frac{\Delta z_{1}}{K_{1, j, 1}^{\mathrm{m}, 1 / 2}\left(p_{1, j, 1 / 2}^{\mathrm{m}}\right)}}
$$

and, the gravitational contribution to the porous medium balance equation, Eq. $3-15$, is :

$$
\begin{aligned}
G_{1, j, \mathrm{k}=1}^{\mathrm{m}}= & A_{\mathrm{k}=1}\left(\frac{2 p_{1, j, 1}^{\mathrm{m}}}{\left(\frac{\Delta z_{1}}{K_{1, j, 1}^{\mathrm{m}, 1 / 2}\left(p_{1, j, 1 / 2}\right)}\right)}+\rho g K_{i, j, 1}^{\mathrm{m}, 1 / 2}\right)+ \\
& A_{\mathrm{k}=1} \rho g\left(\frac{\Delta z_{1}+\Delta z_{2}}{\frac{\Delta z_{1}}{K_{1, j, 1}^{\mathrm{m}, 3 / 2}}+\frac{\Delta z_{2}}{K_{1, j, 2}^{\mathrm{m}, 3 / 2}}}\right)
\end{aligned}
$$

If the boundary pressure $p_{i, j, n+1 / 2}^{m}$ is specified (known) at the right most boundary in the $\mathrm{z}$ direction, $\mathrm{k}=\mathrm{n}+1 / 2$, then the flux,

$$
q_{1, j, \mathrm{n}+1 / 2}^{\mathrm{m}}=-K_{i, j, \mathrm{n}}^{\mathrm{m}, \mathrm{n}+1 / 2}\left(\frac{p_{i, \mathrm{j}, \mathrm{n}+1 / 2}^{\mathrm{m}}-p_{i, \mathrm{j}, \mathrm{n}}}{\Delta z_{\mathrm{n}} / 2}+\rho g\right)
$$

is substituted into the flux balance equation, Eq. 3-15, where the boundary pressure is known. This implies, in the porous medium balance equation, Eq. 3-22, that: $e_{k=n}=0$; the coefficient $f_{n}$ is calculated as for an interior cell; the coefficient of $p_{1, j, k=n}^{m}$ is modified from $e_{n}+f_{n}$ to $e^{\prime}{ }_{k=n}+f_{n}$, where

$$
e_{\mathrm{k}=\mathrm{n}}^{\mathrm{m}^{\prime}}=\frac{2 A_{\mathrm{k}=\mathrm{n}}}{\left(\frac{\Delta z_{\mathrm{n}}}{K_{\mathrm{i}, \mathrm{j}, \mathrm{n}}^{\mathrm{m}, \mathrm{n}+1 / 2}\left(p_{\mathrm{i}, \mathrm{j}, \mathrm{n}+1 / 2}^{\mathrm{m}}\right)}\right)}
$$

and, the gravitational contribution to the porous medium balance equation, Eq. $3-15$, is : 


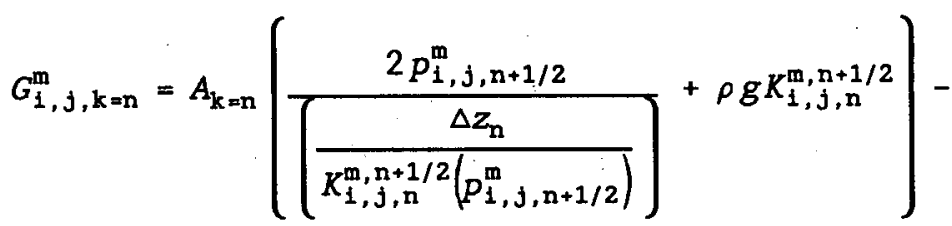

$$
\begin{aligned}
& A_{\mathrm{k}=\mathrm{n}} \rho g\left(\frac{\Delta z_{\mathrm{n}-1}+\Delta z_{\mathrm{n}}}{\frac{\Delta z_{\mathrm{n}-1}}{K_{\substack{\mathrm{m}, \mathrm{n}-1 / 2 \\
j, \mathrm{n}-1}}+\frac{\Delta z_{\mathrm{n}}}{K_{1, j, \mathrm{n}}^{\mathrm{m}, \mathrm{n}-1 / 2}}}}\right)
\end{aligned}
$$

The corresponding pressure boundary condition implementation for the fracture equation is obtained by interchanging the superscripts ' $m$ ' and ' $f$ ' in the coefficients and advection contributions. The implementation of pressure boundary conditions in the other directions is obtained by cyclically interchanging the indices as $k>i>j>k$, taking into account the possible appending of the advection term in the case that gravity is not parallel to the $\mathbf{z}$ direction.

\subsubsection{Time Dependent Volume Sources and Boundary Conditions}

The volume sources, $Q^{m}$ and $Q^{f}$, as well as the boundary pressures and boundary fluxes at the left and right exterior surface boundaries in the $x, y$, or $z$-directions, can each be defined as time dependent from a list of times and values for any applicable spatial subdomain (volume or exterior surface boundary) of the problem.

The volume source terms for the porous medium or fracture are applied only at the $(i, j, k)$ interior grid blocks, where $1 \leq i \leq n_{x}, 1 \leq j \leq n_{y}$, and $1 \leq$ $k \leq n_{z}$. Here, $n_{x}, n_{y}$, and $n_{z}$ are the number of cells in the $x, y$, and $z$ directions, respectively. The sources may be prescribed explicitly or distributed (by the code) between the porous medium and fracture based on the ratio of the porous medium and fracture mobility coefficients.

The boundary pressures and fluxes are applied only at the exterior surfaces [left, right] given by:

$$
\begin{aligned}
& x:\left[(1 / 2, j, k),\left(n_{x}+1 / 2, j, k\right)\right], \\
& y:\left[(i, 1 / 2, k),\left(i, n_{y}+1 / 2, k\right)\right], \text { and } \\
& z:\left[(i, j, 1 / 2),\left(i, j, n_{z}+1 / 2\right)\right] .
\end{aligned}
$$

On each surface, the boundary conditions may be prescribed on any defined, existent, rectangular subdomain of interest. The fluxes or pressures may also be distributed (by the code) between the porous medium and fractures using a mobility ratio between the porous medium and fracture.

In every instance, the source term or boundary condition time dependence is prescribed by linear interpolation from a table of values and times, $T_{r}$, where $1 \leq r \leq R_{\max }$, and $R_{\max }$ is the number of elements in a set. The values used by the code depend on the value of the time $t$ relative to $T_{r}$. If $t<$ $T_{1}$ then a default value, usually 0 , is used. If $T_{r} \leq t \leq T_{r+1}$, and $0 \leq r+1$ 
$\leq R_{\max }$, then linear interpolation is applied over that subinterval. If $t \geq$ $\mathrm{T}_{\mathrm{Rmax}}$, then the last value supplied in the table is used. 

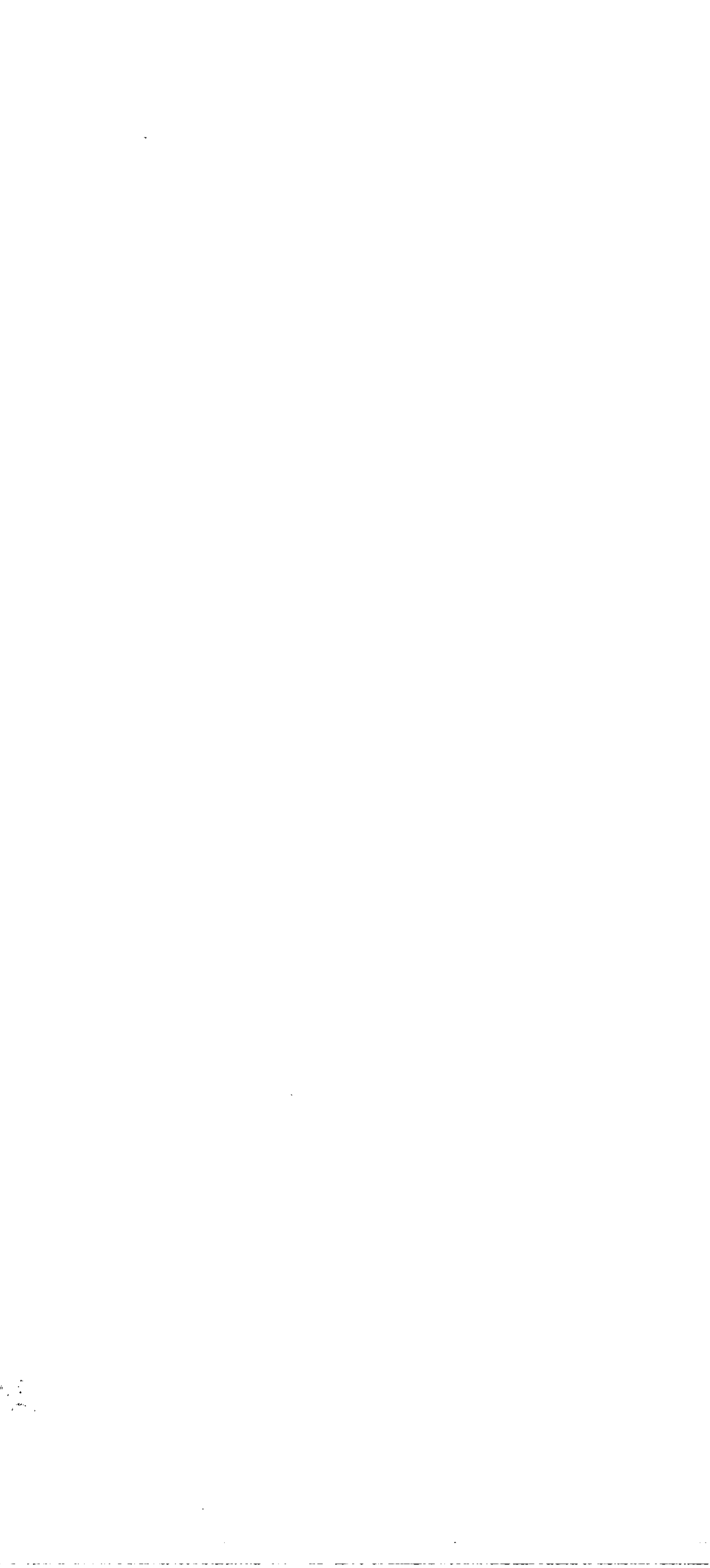
This section describes the basic structure of DCM3D. The code is written in ANSI standard FORTRAN. All variables, function subprograms, character strings, and parameters are declared. All intrinsic functions are generic, ie. the argument type (integer, real, double precision) determines the function type. The names of all variables, parameters, function and subroutine subprograms are limited to six characters. The use of common blocks is minimized as much as possible.

Even though it was written on a CRAY X-MP computer, DCM3D should compile on all types of computers. However, DCM3D calls some system dependent routines that return dates, clock times, and running times. These routines should be replaced in order to create an executable version of the computer program.

\subsection{Main Driver}

Described in this section is the main driver of the flow code. The main program serves as the driver of the flow code. It calls all the subroutines necessary to perform the calculations. A flow chart of the main driver appears in Figure 4-1. A summary of each item in the flow chart follows.

Declare variable types: Each variable, constant, or parameter name used globally throughout the code is typed as to whether it is a character, integer, or real type. Characters as a group are typed first, integers second, and reals third. Names within each type are typed in alphabetical order. Variable and constant names local to a subprogram are only typed in the appropriate subprogram.

Set parameters: Parameters are used to dimension most of the arrays in the code. The parameters occur in two groups. The first group is explicitly set with constants. This group is used to set array sizes for grid spacing, boundary conditions, source terms, and writing output. The second group of parameters is calculated from the first group. This group is used to dimension integer and real working arrays used by LSODES, the main differential equation solver in DCM $3 D$, and to dimension the independent variables.

Dimension variables: All variables used globally are dimensioned. They are dimensioned in alphabetical order without regard to type. Most variable dimensions are based on the parameter values set above. It is necessary to change only a few parameters in order to dimension all arrays in the code.

Set up common blocks: Common blocks shared only with subroutine DYDTEQ, the differential equation generating subroutine called by subroutine LSODES, are declared. The common blocks are declared in alphabetical order. Most variables in DCM3D are transferred between subprograms by subprogram call statements. However, subroutine LSODES calls subroutine DYDTEQ with no allowance made for an argument 


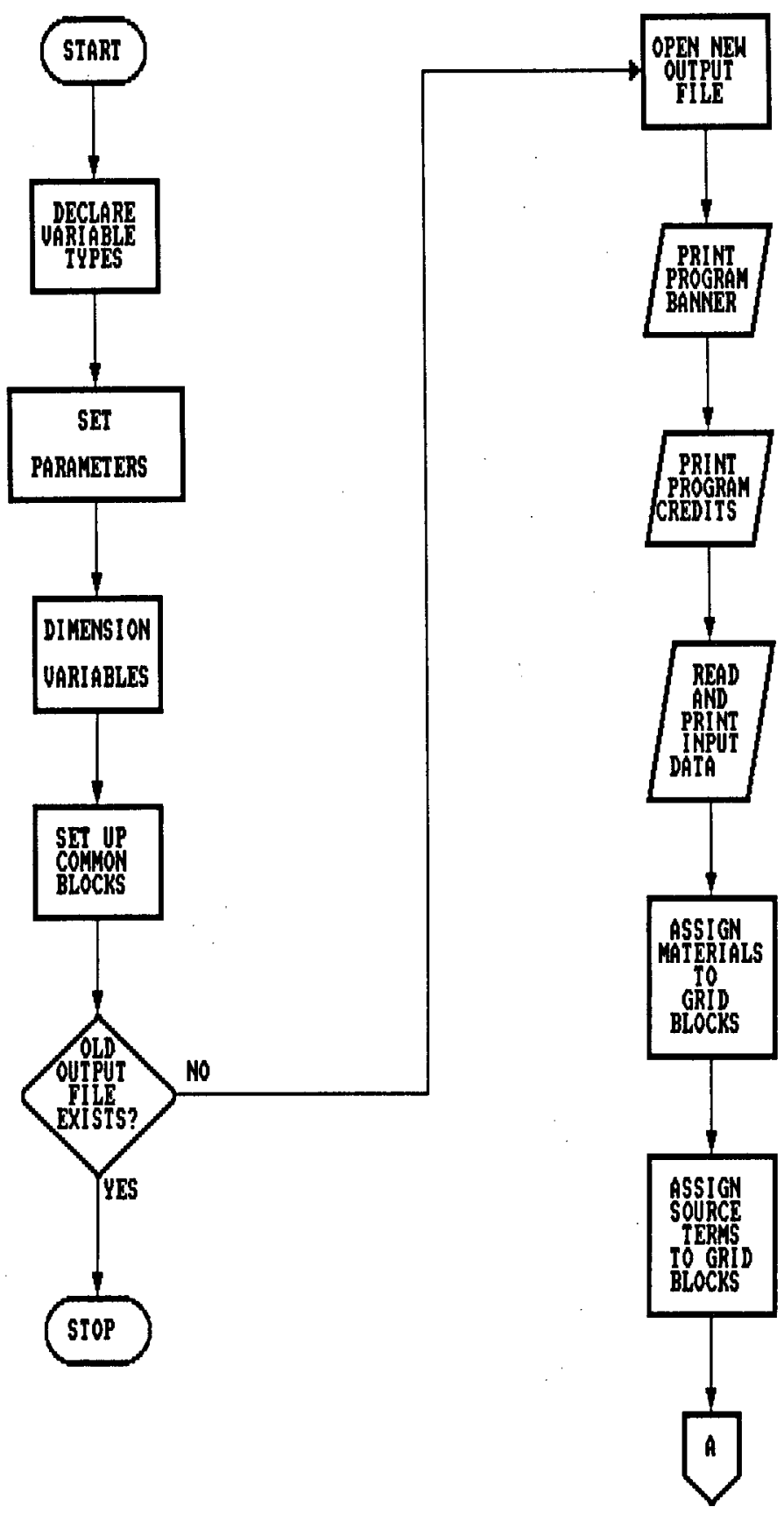

Figure 4-1: Flow Chart of DCM3D Main Driver 


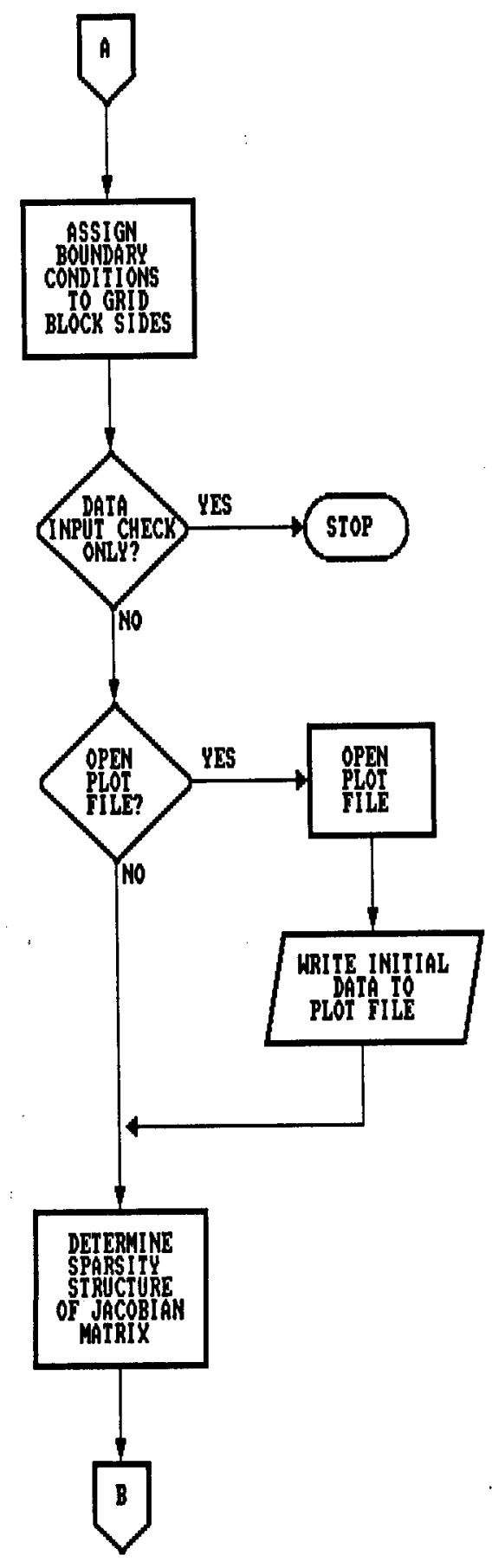

Figure 4-1: Flow Chart of DCM3D Main Driver (continued) 


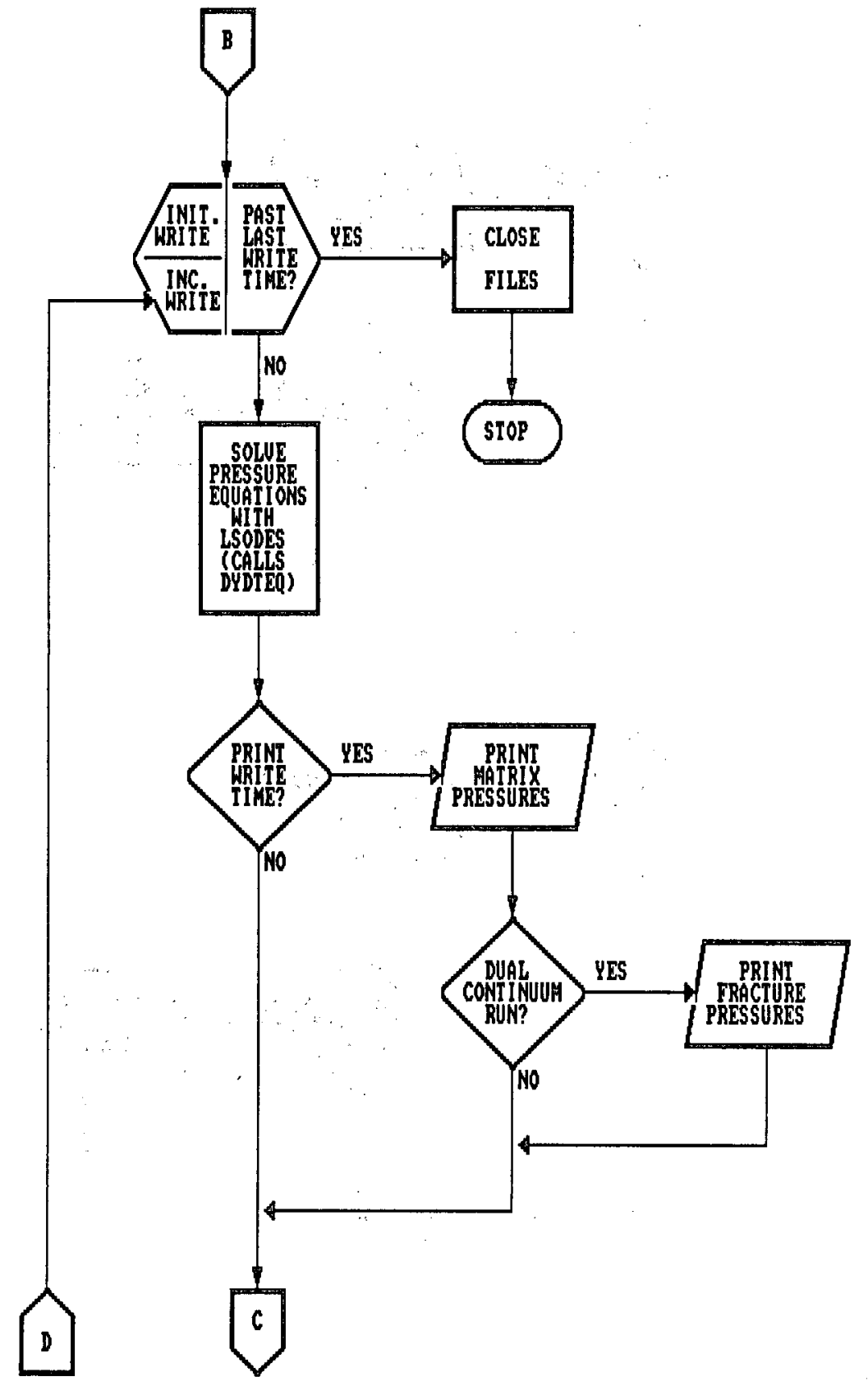

Figure 4-1: Flow Chart for DCM3D Main Driver (continued) 


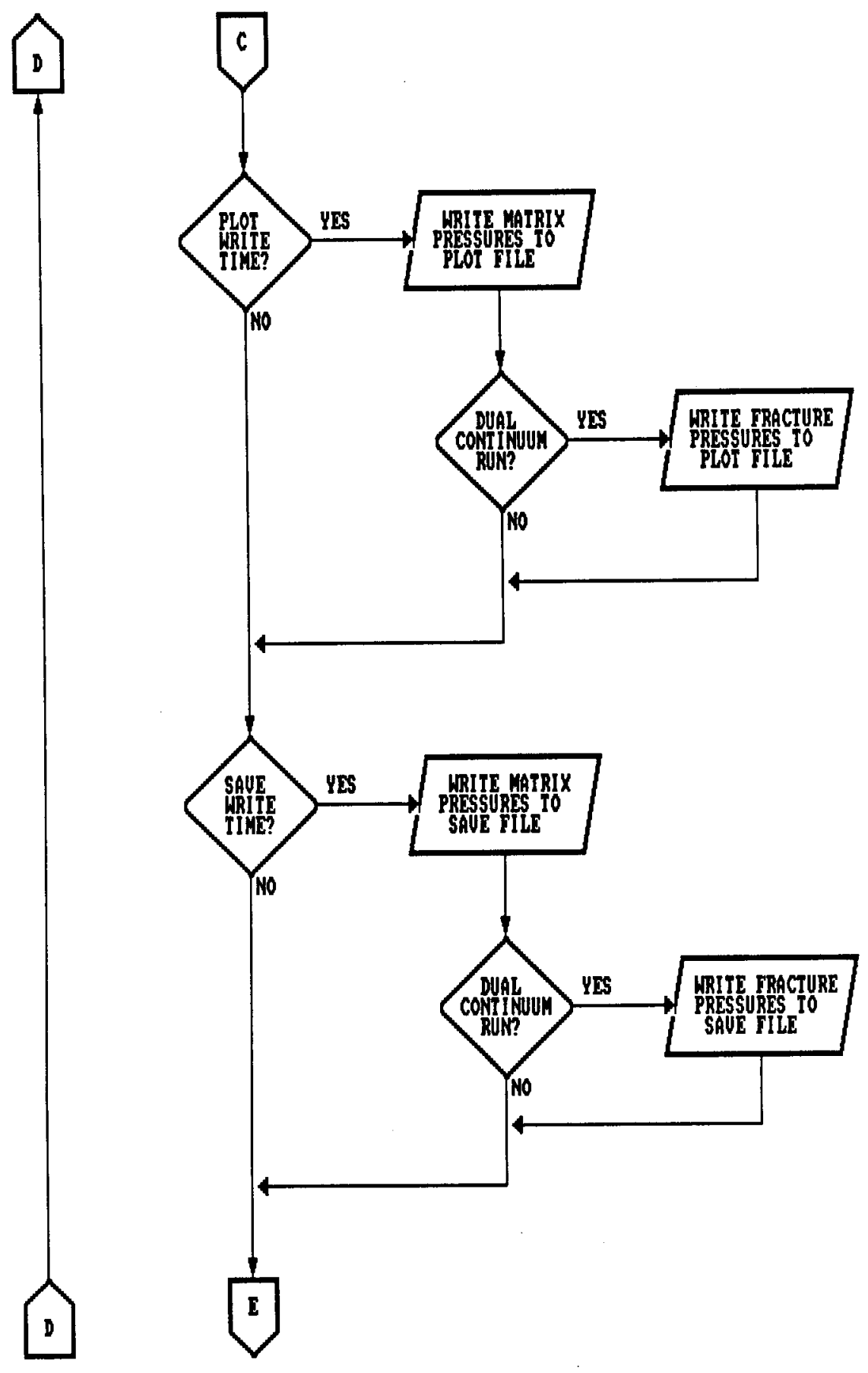

Figure 4-1: Flow Chart for DCM3D Main Driver (continued) 


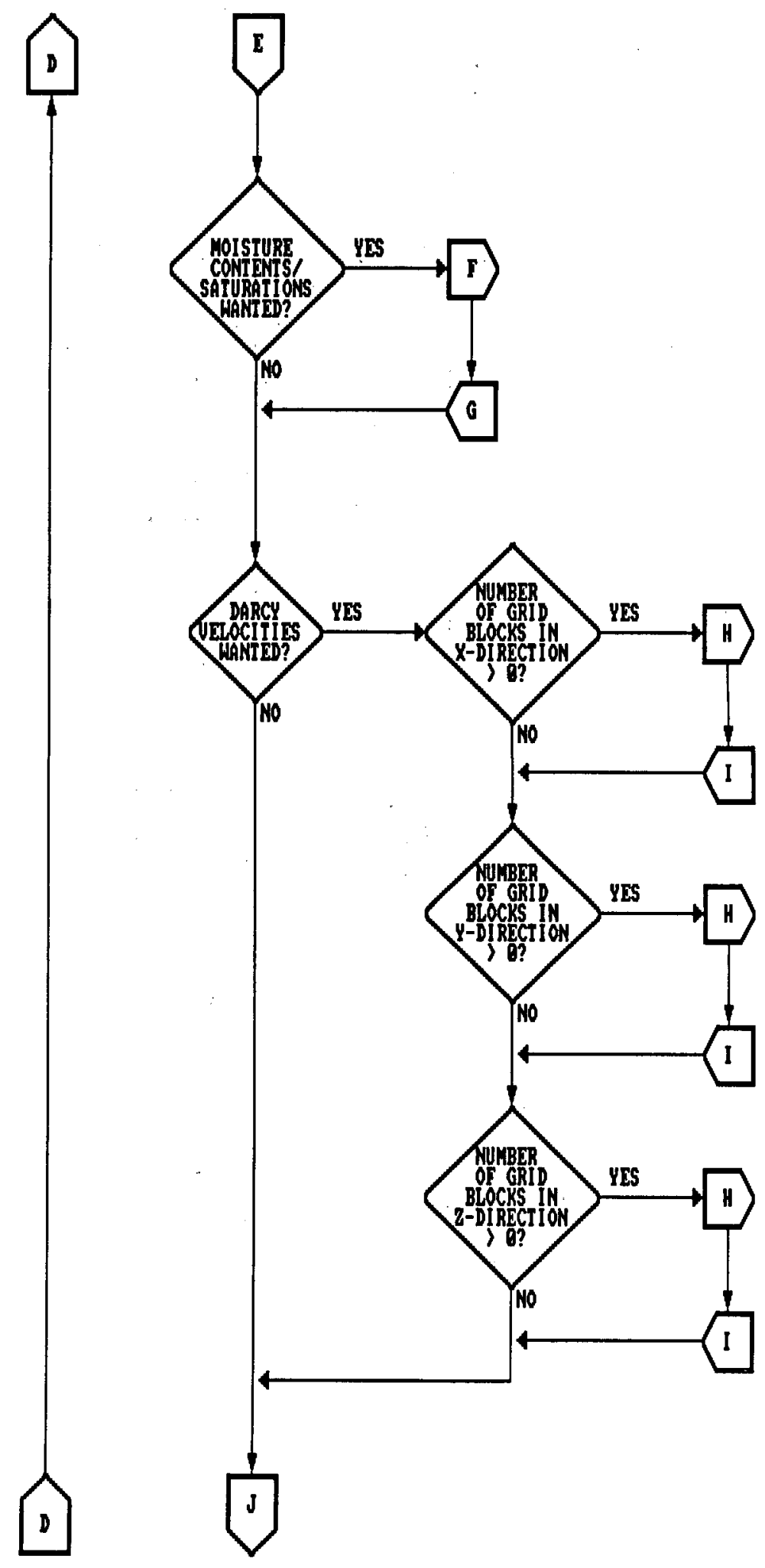

Figure 4-1: Flow Chart for DCM3D Main Driver (continued) 


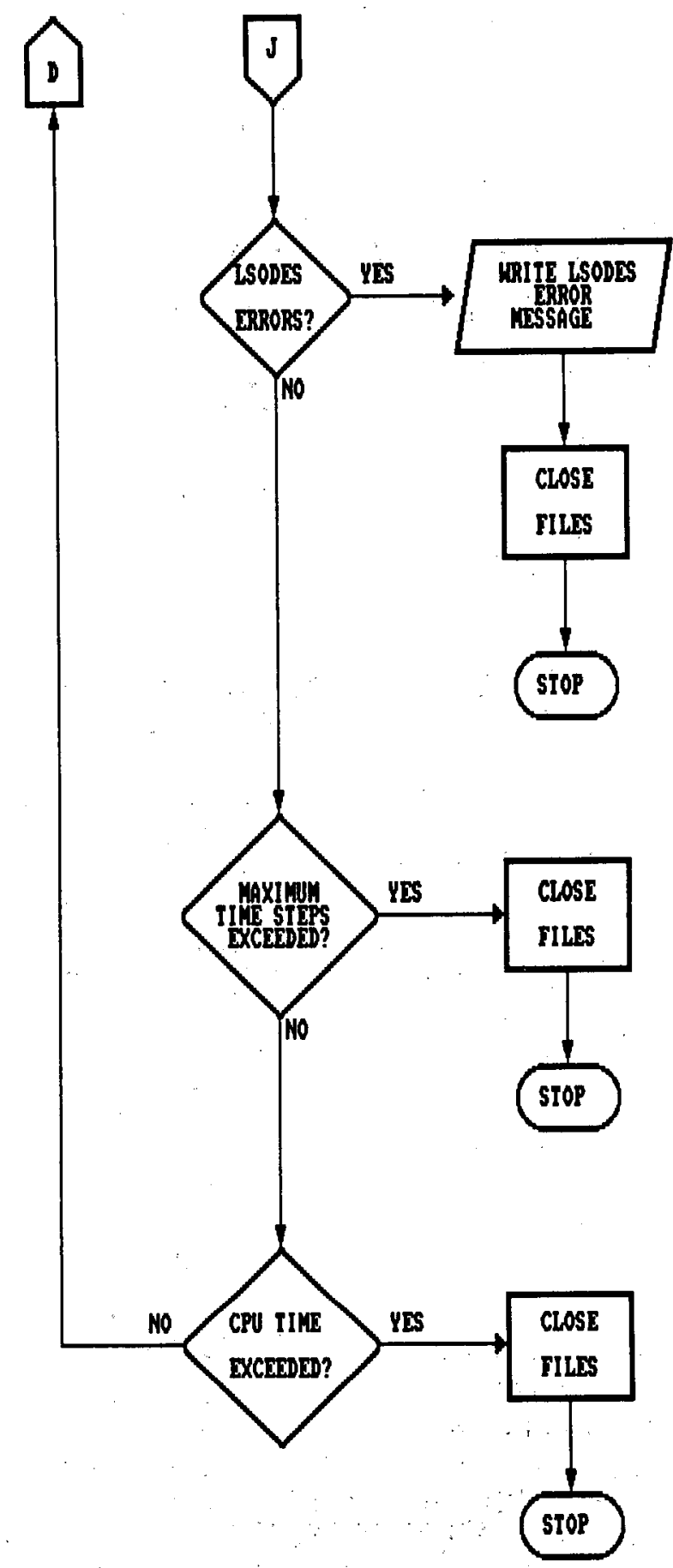

Figure 4-1: Flow Chart for DCM3D Main Driver (concluded) 
list for transferring variables. Thus, common blocks present the only way to transfer variables into subroutine DYDTEQ without modifying subroutine LSODES.

old output file exists?: The code checks to determine if an old output file exists. If an old output file exists, it probably was created from a previous run. In order to avoid overwriting a file that may be useful at a later time, DCM3D stops running and prints a message. If no output file exists, the code continues running.

Open new output file: If an old output file does not exist, a new one is opened here.

Print program banner: A program banner is written to the output file. Subroutine BANNER writes the banner.

Print program credits: A disclaimer indicating that the government is not responsible for errors and omissions in DCM $3 D$ is written to the output file. Subroutine CREDIT writes the disclaimer.

Read and print input data: DCM3D checks for the existence of an input file. If no input file exists, DCM3D stops running. Otherwise, the code continues. The code reads the input file line by line as character strings. The strings are printed line by line with line numbers. Subroutine ECHO performs this task. After the echo, the input file is read as formatted input. Each line is output as formatted output. If a read error occurs due to an improperly formatted input line, an error message, giving the line number of the error; is generated. This directs the analyst where to look in the input file to correct any input data errors. In addition, the existence of a plot file is checked. If a plot file exists, DCM3D prints a message and stops running. Subroutine IODATA drives the checking of the existence of files, and the reading and writing of the formatted input data. It calls subroutines IOBND, IOGRID, IOMAT, IOPRES, and IOSRC, which read and write boundary condition data, grid data, material data, initial pressure data, and source term data, respectively. Subroutine INERR is responsible for printing error messages regarding any errors while reading the input file. These subroutines also check the values of selected parameters set in the main program to determine if certain arrays are large enough for the problem. Subroutine PARCHK performs the parameter checking and stops DCM $3 D$ if arrays are not large enough.

Assign materials to grid blocks: Various material types are assigned to all the grid blocks. A check is then made to determine if all grid blocks have a material assigned. If all grid blocks have a material, the code continues. If not, a list of the grid blocks with no assigned materials is printed and the code stops. Subroutine MATCHK performs this task.

Assign source terms to grid blocks: A source term table number is assigned to each grid block. The default is no table number, which implies a zero valued source term for that grid block. Subroutine SRC performs this task. 
Assign boundary conditions to grid blocks sides: Boundary condition table numbers are assigned to the various grid blocks that appear on the grid's boundaries. The default is no table number, which implies a zero flux boundary condition. Subroutine BC performs this task.

Data input check only?: If the purpose of the run is to check the input data only, the code stops running. Otherwise, the code starts to perform the flow calculations.

Open plot file?: If a plot file is desired, it is created. In addition, some preliminary data such as problem title and subtitle, grid spacing, and material data are written to the plot file. If a plot file is not desired, DCM3D continues running. The plot file serves several purposes. It is usually used to generate plots of variables calculated by the code. It can also be used to store calculated variables for use by any code coupler programs. It should be noted that a check for the existence of the named plot file is made in subroutine IODATA (see Read and print input data on the previous page). If the check reveals that the named plot file already exists, DCM3D prints a message and stops running. DCM3D stops running in order to prevent overwriting a plot file that may be used later.

Determine sparsity structure of Jacobian matrix: The sparsity structure of the Jacobian matrix that LSODES uses to solved the set of ordinary differential equations is determined here. Since most of the elements of the Jacobian matrix are zero valued, computer storage is reduced by only saving the nonzero elements of the Jacobian matrix. Because the nonzero elements have a repeating structure, the code can determine the sparsity structure much faster than LSODES. Subroutine SPARSE is used to determine the sparsity structure.

The following steps are performed in a loop.

Increment write time: The time at which calculated output is desired is determined. . If the last write time is exceeded, the calculations are completed and DCM3D closes all files and stops running.

Solve pressure equations with LSODES: The program control is passed to subroutine LSODES. Subroutine LSODES solves for the pressure at the desired write time by internally generated time steps. Subroutine DYDTEQ generates the finite difference equations that Subroutine LSODES solves. Subroutines LSODES and DYDTEQ are described in Section 4.2 of this report.

Print write time?: If this is a print write time, the porous medium pressures and fracture pressures for a dual continuum run are written to the output file for this time. Subroutine OUTPRN performs this task.

Plot write time?: If this is a plot write time, the porous medium pressures and fracture pressures for a dual continuum run are written to the plot file for this time. Subroutine OUTPLT performs this task. 
Save write time?: If this is a save write time, the save file is written or overwritten. The problem title and subtitle, analyst, water properties, gravitational coefficients, convergence tolerances, grid spacing, material data, and the new porous medium pressures and fracture pressures for a dual continuum run are written to the save file for this time. Subroutine IOSAVE performs this task.

Moisture contents/saturations wanted?: If desired at this write time, the porous medium and fracture moisture contents or saturations are calculated. Figure 4-2: shows the sequence for calculating and writing the moisture contents or saturations. Subroutine MOISTR determines whether moisture contents or saturations are desired and performs the appropriate calculations. Subroutines OUTPRN and OUTPLT write the moisture contents or saturations to the output and plot files, respectively.

Darcy velocities wanted?: If desired at this write time, the porous medium and fracture Darcy velocities at the interfaces between grid blocks are calculated for this write time. Darcy velocities are only calculated in a directions where one or more grid blocks exist in that direction. Figure 4-3 shows the sequence of calculating and writing the Darcy velocities. Subroutine DARCY performs the calculation of the Darcy velocities. Subroutines OUTPRN and OUTPLT write the Darcy velocities to the output and the plot files, respectively.

LSODES errors?: If the LSODES solver indicates to DCM3D that the equations cannot be solved for a particular time step, DCM3D prints a message to that effect, closes any open write files, and stops running.

Maximum time steps exceeded?: If the maximum number of time steps for the problem is reached or exceeded, DCM3D prints a message, closes any open write files, and stops running.

CPU time exceeded?: If the maximum amount of CPU time for the problem is reached or exceeded, DCM3D prints a message, closes any open write files, and stops running.

After the CPU time limit check, the loop is repeated until the final write time. If the final write time has been reached, DCM3D closes all files and stops running.

\subsection{General Description of the LSODES ODE Solver}

The spatial operators of the original ground-water flow equations (Eq. 3-1 and Eq. 3-2) were approximated by finite difference methods (Section 3.2), resulting in a system of equations of the form:

$$
\frac{\partial \bar{y}}{\partial t}=\bar{f}(\bar{y}, t)
$$




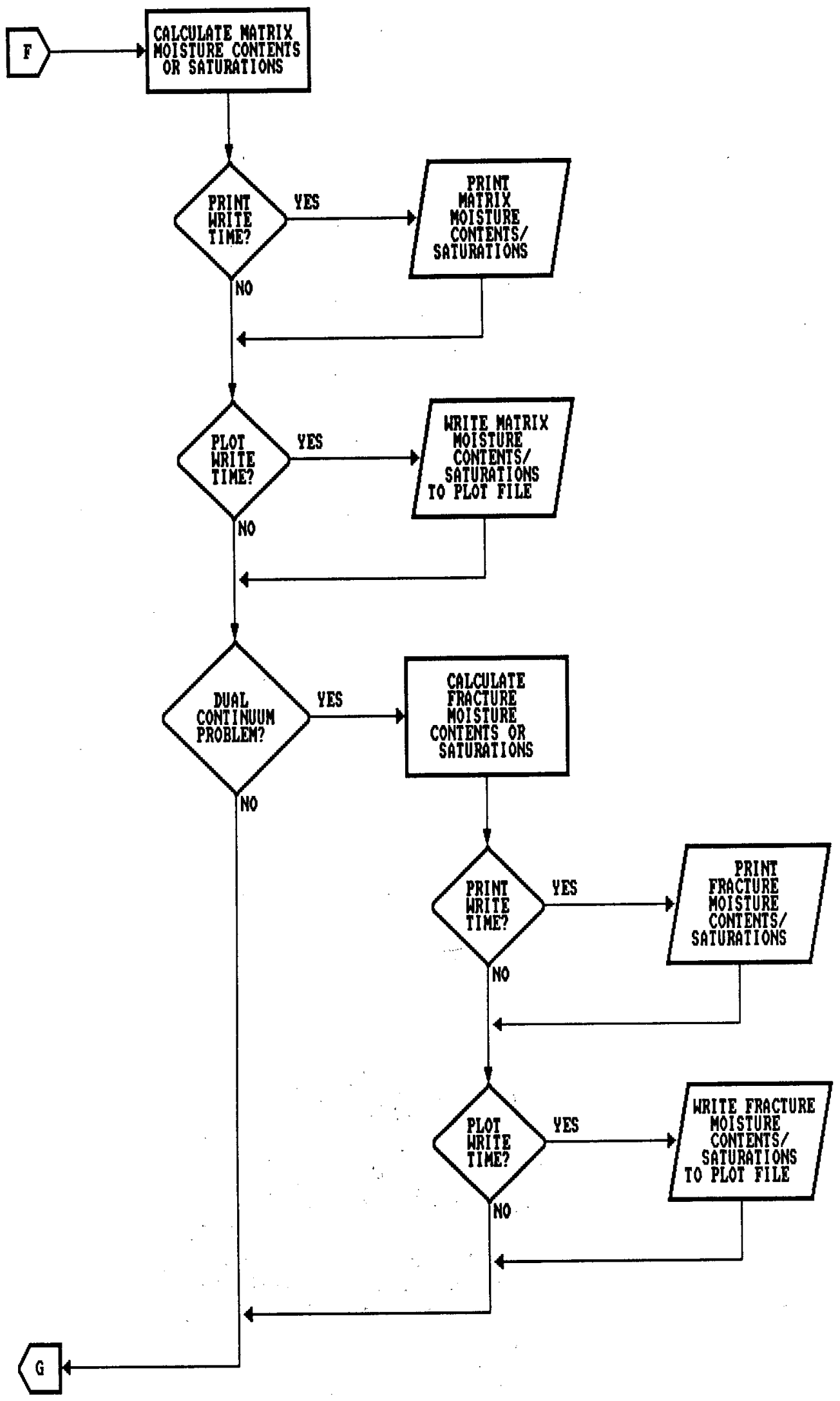

Figure 4-2: Flow Chart for DCM3D Moisture Content/Saturation Calculations 


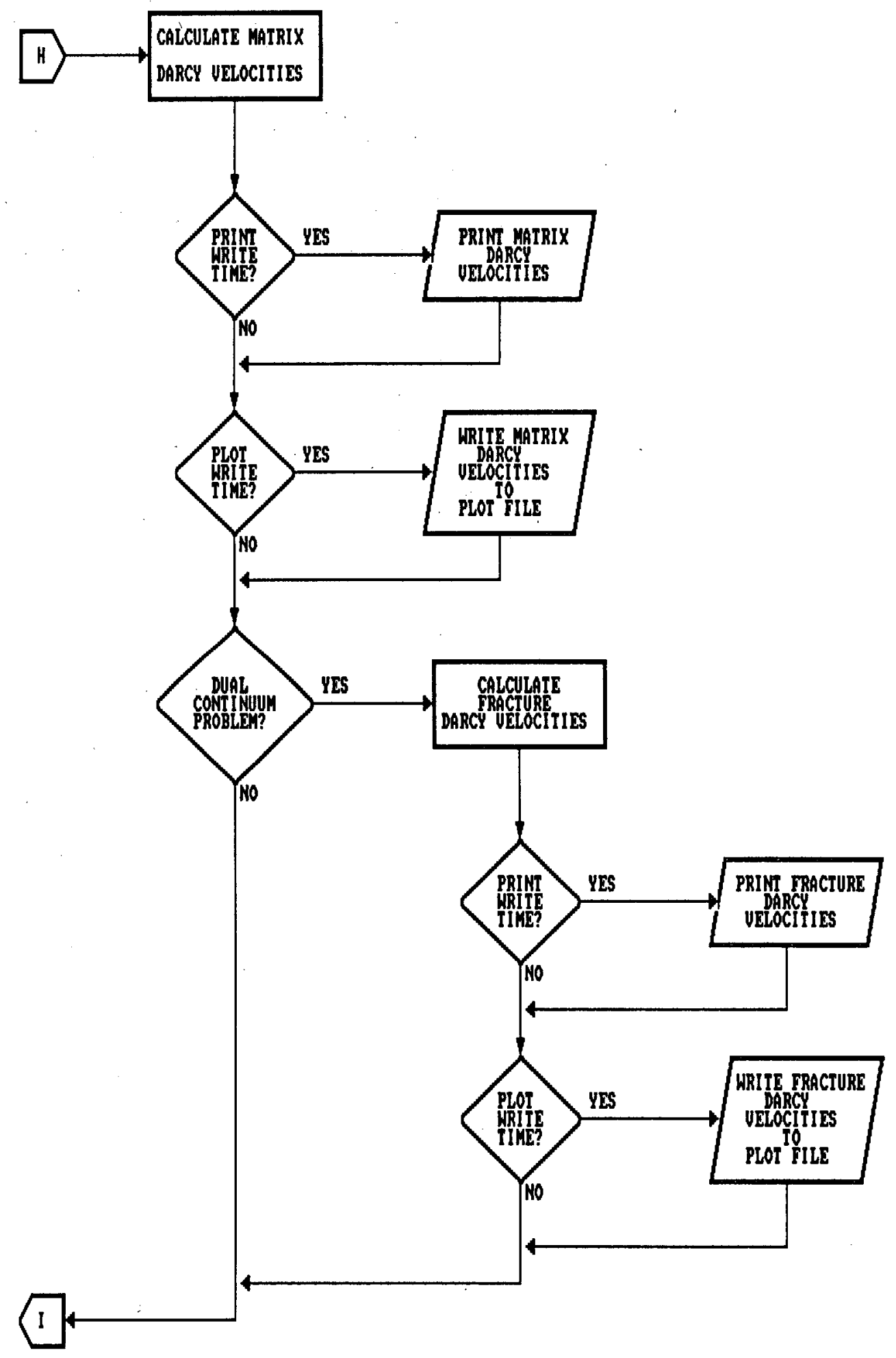

Figure 4-3: Flow Chart for DCM3D Darcy Velocity Calculations - Page 40 - 
where $\bar{y}$ is the vector of dependent variables, $\bar{f}(\bar{y}, t)$ is a vector function of the dependent and independent variables, and $t$ is the independent variable. The system of equations for the fractured porous medium flow model forms a highly coupled set of stiff, ordinary differential equations (ODE's).

DCM3D uses the LSODES program, developed by Hindmarsh (1983), to solve the ODE's constructed for the fractured porous medium flow model. LSODES requires that equations of the form of Eq. 4-1 to be evaluated at time, $t$, by the solver routine. These equations are generated by the DYDTEQ subroutine in the code. The overall logic used for implementing these ODE's into the subroutine DYDTEQ is summarized in Figure 4-4 and Figure 4-5.

In Figure 4-4 and Figure 4-5, the following definitions and conventions are used:

\begin{tabular}{|c|c|c|}
\hline Adv & $=$ & $\begin{array}{l}\text { the advection term contributions that arise from } \\
\text { the gravitational }(\rho \mathrm{g}) \text { terms in the flux }\end{array}$ \\
\hline Diag & $=$ & $\begin{array}{l}\text { equation, } q=-K(\nabla p+\rho g \nabla z) \\
\text { the diagonal contributions to the coefficient for } \\
\text { the cell }(i, j, k) \text {. }\end{array}$ \\
\hline So & $=$ & $\begin{array}{l}\text { source term contributions due to a specific } \\
\text { boundary condition. }\end{array}$ \\
\hline Vo1 & $=$ & volume of the $(\mathbf{i}, \mathbf{j}, \mathbf{k})$ cell. \\
\hline $\mathrm{D} 1, \mathrm{D} 2$ & $=$ & $\begin{array}{l}\text { cell spacings in } x, y \text {, or } z \text { direction for current } \\
\text { cell. }\end{array}$ \\
\hline$D(1), D(N)$ & $=$ & $\begin{array}{l}\text { first and last cell spacings in } x, y \text {, or } z \\
\text { directions. }\end{array}$ \\
\hline Psi & $=$ & $\begin{array}{l}\text { outside cell boundary surface pressure, a function } \\
\text { of time. }\end{array}$ \\
\hline q_bdry & $=$ & $\begin{array}{l}\text { outside cell boundary surface flux, a function of } \\
\text { time. }\end{array}$ \\
\hline$a a, b b$ & $=$ & $\begin{array}{l}\text { coefficients multiplying the pressure, modified for } \\
\text { boundary conditions at cell boundary surface. }\end{array}$ \\
\hline$w 1, w 2$ & $=$ & $\begin{array}{l}\text { coefficients multiplying the pressure for internal } \\
\text { cells. }\end{array}$ \\
\hline
\end{tabular}

The convention used in Figure 4-5 for coefficient(w), $w=x, y$, or $z$, is that $\operatorname{coef}(w+)=w 2$ and $\operatorname{coef}\left(w_{-}\right)=w 1$. In terms of the notation of the difference equations in Section 3, this is equivalent to

$$
\begin{aligned}
& x \text { direction : } a=\operatorname{coef}(x+) \text { and } b=\operatorname{coef}\left(x^{-}\right) ; \\
& y \text { direction }: c=\operatorname{coef}(y+) \text { and } d=\operatorname{coef}(y-) ; \text { and } \\
& z \text { direction }: e=\operatorname{coef}(z+) \text { and } f=\operatorname{coef}\left(z^{-}\right) ;
\end{aligned}
$$

where $a, b, c, d, e$, and $f$ are the coefficients defined in Eq. 3-18, Eq. 3-19, and Eq. 3-20. The $+/$ - notation used for $\operatorname{coef}(w)$ is associated with the $i, j$, or $\mathrm{k}$ indices in a particular direction:

$$
\begin{aligned}
& x \text { direction }:+:(i+1, j, k) \text { and }-:(i-1, j, k) ; \\
& y \text { direction }:+:(i, j+1, k) \text { and }-:(i, j-1, k) ; \text { and, } \\
& z \text { direction }:+:(i, j, k+1) \text { and }-:(i, j, k-1) \text {. }
\end{aligned}
$$




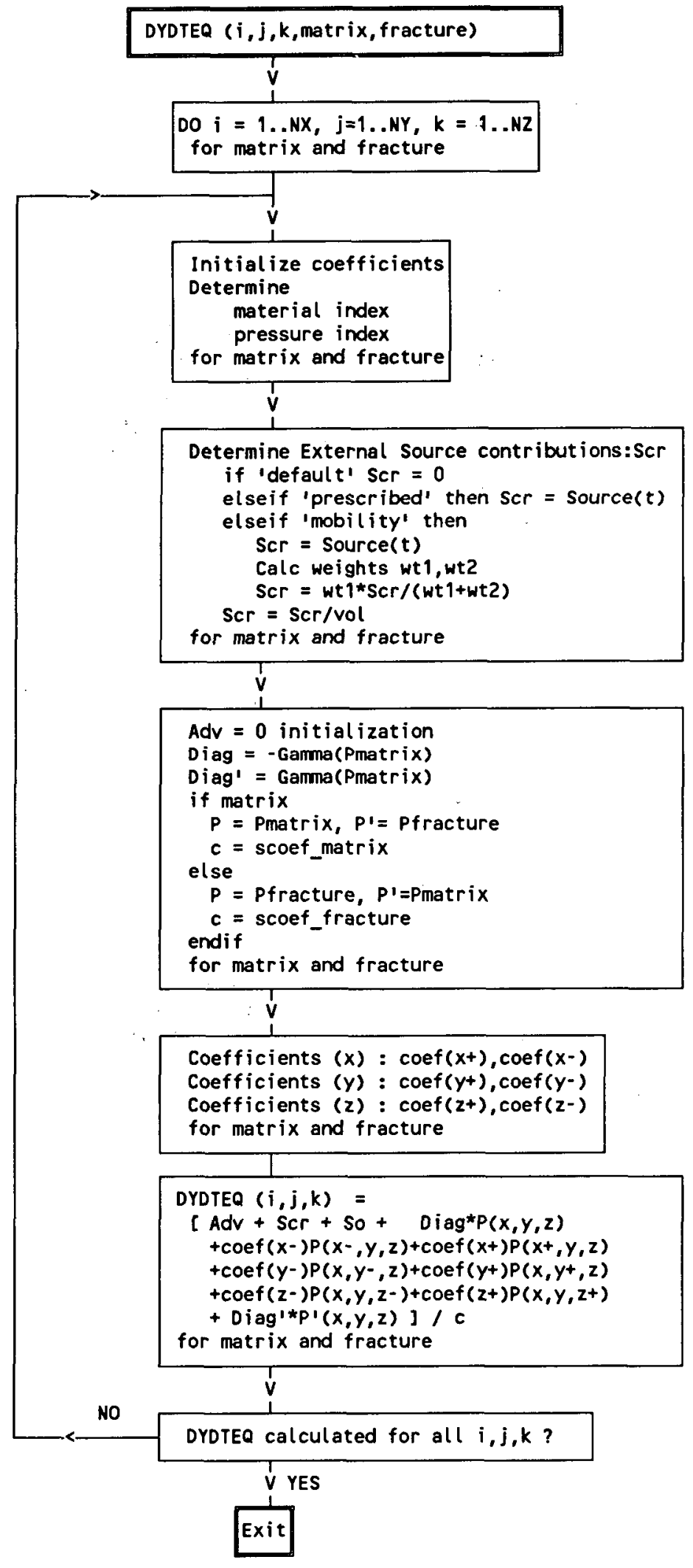

Figure 4-4: Flow Chart for Subroutine DYDTEQ 


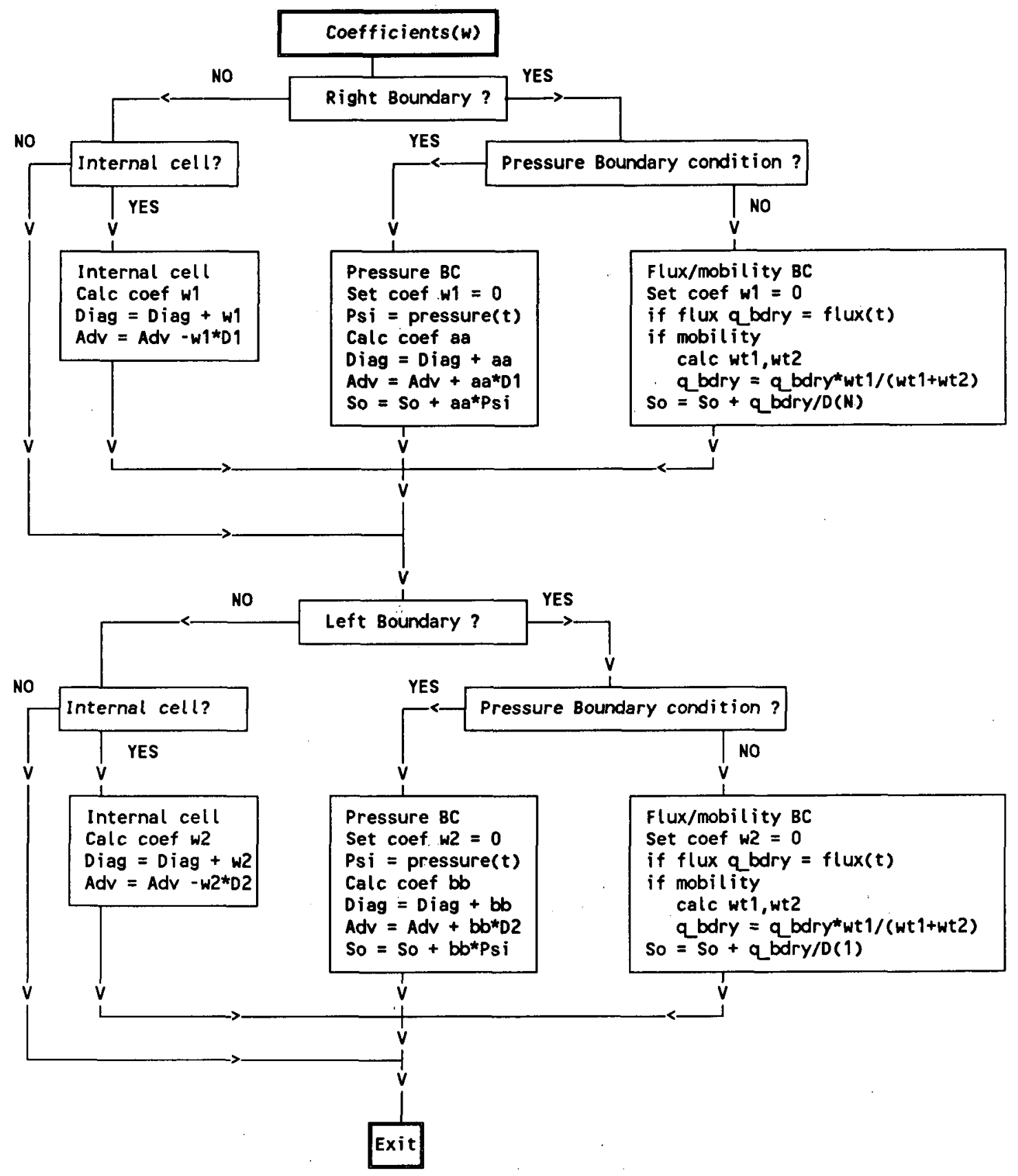

Figure 4-5: Coefficient Evaluation Logic for Subroutine DYDTEQ 
If only a one- or two-dimensional problem is being solved, the coefficients for the omitted dimensions default to zero values.

The LSODES program is one of the programs contained in ODEPACK (Hindmarsh, 1983). ODEPACK contains a collection of FORTRAN solvers for the initial value problem (Eq. 4-1) for ODE systems. It includes six solvers, suitable for both stiff and non-stiff systems, and includes solvers for systems of equations given in linearly implicit form as well as solvers for systems given in explicit form. The source code for these solvers is written in FORTRAN IV with minimal machine dependencies, which are detailed in the documentation contained in the source listings. Each solver consists of a driver program having the same name as the solver (for example LSODES), and a number of subordinate routines.

After performing benchmarking of ODEPACK and investigating each of the available solvers on a number of known stiff problems, the LSODES solver was determined to be best suited for solving this class of porous mediumfracture transport initial value problems. LSODES solves the systems $\partial \bar{y} / \partial t=f(\bar{y}, t)$ in the stiff case by treating the Jacobian matrix in general sparse form. The specific sparsity structure information is supplied by the code to LSODES for improved computational performance. Parts of the Yale Sparse Matrix Package (YSMP) are used by LSODES to solve the linear systems that arise. The LSODES package supersedes, and improves upon, the older GEARS packages. The installed LSODES and supporting routines contain approximately 11,000 lines of FORTRAN source code. The source code for the ODEPACK programs was obtained directly from Dr. A. C. Hindmarsh on floppy diskettes, which were installed, used, benchmarked, and archived at SNL in 1989.

The ODEPACK programs were installed and run on the SNL CRAY with single precision arithmetic and on 80386-based personal computers with 4 megabytes of extended memory, a Lahey F77L-EM/32 FORTRAN compiler, and double precision arithmetic. The general installation instructions, recommended usage, and a discussion of the specific non-ANSII standard features of the package are included in the ODEPACK source code listings and documentation. 


\subsection{INSTRUCTIONS FOR RUNNING DCM3D}

Section 5.0 of this report describes the data requirements and data input structure for the flow code. Data requirements are described in section 5.1. Specific instructions for setting up an input file are described in Section 5.2. Instructions for a restart run are presented in Section 5.3. Dimensioning of arrays is described in Section 5.4. Section 5.5 tells the analyst about running DCM3D with pressure head instead of pressure.

Section 5.6 provides a description of the plot file. Section 5.7 provides the analyst with general compiling and linking instructions for DCM3D.

\section{$5.1 \quad$ Required Data}

Data needs for DCM3D consist of two types. The first type is the nonhydrologic parameters necessary to run the code. This group includes items such as title, convergence criteria, and time step size. The second type consists of hydrologic data such as permeability, porosity, transfer coefficient, and initial conditions.

\subsubsection{Non-hydrologic Data}

The non-hydrologic data records are summarized below. The data are grouped and presented in the order in which they are read in the code.

TITLE provides a name for the problem.

SUBTITLE provides additional information about the problem.

ANALYST is the name of the person running the particular problem.

FILES provides names for the plot and the save files.

OPTIONS provides information on how the code is to handle some aspects of the problem such as solving equations for a single or double porosity medium, or just checking the input data.

WATER DATA consists of the water density, water dynamic viscosity, and water bulk modulus of compressibility.

GRAVITY DATA consists of the gravitational coefficient and a vector describing the gravity vector.

CONVERGENCE CRITERIA provides a description of the relative and absolute convergence criteria required by Subroutine LSODES, and a convergence criteria for the Newton-Raphson iteration of the interface pressure for the interface permeability calculations.

TIME STEP DATA provides information on maximum time steps for a problem, maximum time steps between output writes, start time for the simulation, initial time step size, minimum and maximum time step size, and permissible amount of CPU time to use. 
GRID BLOCK DATA provides information on the number of grid blocks in each direction and the size of each grid block.

WRITE TIMES tells the code at which times to print results or write results to plot or save files. It also tells the code whether or not to calculate Darcy velocities, and moisture contents or saturations, and, if calculated, at which times to print them.

\subsubsection{Hydrologic Data}

The hydrologic data records are summarized below. The data are grouped and presented in the order in which they are read in the flow code.

MATERIAL DATA provides the code with information on the location of the various soil and rock types within the grid and the hydrologic properties of both the porous medium and the fracture continua.

The hydrologic material properties for the porous medium are provided on two records. On the first record are the bulk modulus of compressibility, porosity, and the anisotropic permeability in the $x-, y-$, and $z-$

directions. The second record provides the data for the van Genuchten characteristic curves, which describe the capillary pressure-degree of saturation and the relative hydraulic conductivity-capillary pressure relationships.

The hydrologic properties needed to describe the fractures are similar to those needed to describe the porous medium. However, the first fracture data record also includes the porous medium to fracture transfer coefficient. The fracture properties from the first record are average values over the entire grid block volume. The data for the fracture continuum are not necessary if a single continuum problem is being run.

INITIAL PRESSURE CONDITIONS provide a starting point of pressure for the code to simulate pressure changes due to infiltration or other stress to the flow field. Pressures are negative if a grid block is located in the unsaturated zone and positive if located in the saturated zone. An initial pressure is provided for each grid block.

X-DIRECTION BOUNDARY CONDITIONS provide information on the boundary conditions on the two faces whose normal vectors point in the $x$-direction. In general, the left face of a boundary is at the origin and the right face is away from the origin. The code is capable of handling both prescribed flux and prescribed pressures on the same face. The boundary conditions can vary in both time and space. Data are required to provide the location of each type of boundary condition on the face. The user describes the time dependence of the boundary conditions in a table. The value of the boundary condition is interpolated from the table for the simulated time of interest. The flux boundary conditions can be described separately for the porous medium and fractures or made to depend on the mobility coefficient of the porous medium and fractures. The pressure boundary conditions can be described separately or set to the same values for the porous medium and fractures. 
Y-DIRECTION BOUNDARY CONDITIONS provide information on the boundary conditions on the two faces whose normal vectors point in the y-direction. The data needs for the y-direction boundary conditions are the same as those needed for the $\mathrm{x}$-direction boundary conditions.

Z-DIRECTION BOUNDARY CONDITIONS provide information on the boundary conditions on the two faces whose normal vectors point in the $z$-direction. The data needs for the $z$-direction boundary conditions are the same as those needed for the $\mathrm{x}$-direction boundary conditions.

SOURCE TERM provides a description of any sources or sinks within the modeled region. An example of a source is a recharge well. An example of a sink is a pumping well. The source term can vary both in space and time. The location of each grid block with a source term is provided by the user. The time dependence of each source term is provided by the user in a table. The value of the source term is interpolated from the table for the simulated time of interest. The source term can be described separately for the porous medium and fractures or made to depend on the mobility coefficient of the porous medium and fractures.

\subsection{Data Input for an Initial Run}

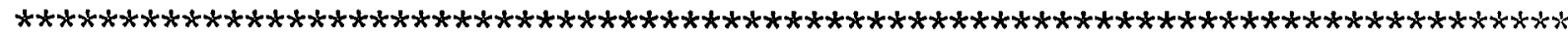

Group A: Title Record

Subgroup A1: Title

Variables: TITLE

Format: $\quad$ A80

Description: $\quad$ TITLE is the name of the problem. If TITLE is blank, the problem will not run.

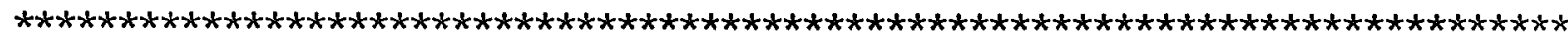

Group B: Subtitle Record

Subgroup B1: Subtitle

Variables: SUBTIT

Format: $\quad$ A80

Description: $\quad$ SUBTIT provides supplementary information about the problem title. A record is required but it may be blank.

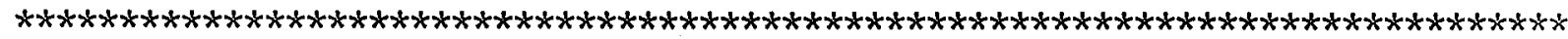

Group C: Analyst Record

Subgroup C1: Analyst

Variables: $\quad$ ANALST

Format: $\quad$ A80

Description: ANALST is the name of the person running the problem. If ANALST is blank, the problem will not run.

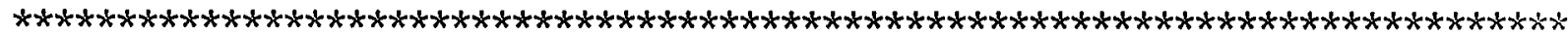


Subgroup D1:

Variables:

Format:

Description:
Files

FILPLT, FILSAV

2A10

FILPLT is the name of the file to which plot data are to be written. A plot file is needed if the output is to be used for some data manipulation process, such as a plot or a post-processor. If FILPLT is blank, no plot file will be created. If a plot file with the name specified by FILPLT already exists, DCM3D will print a message to that effect and will stop running. In order to run the problem, either the name of the plot file or FILPLT must be changed. This check is designed to prevent accidental destruction of a file that may be needed later. Output data that go into the plot file is specified under Group K.

FILSAV is the name of the file to which save data are to be saved for a later restart run. If FILSAV is blank, no save file will be created. If a save file with the name specified by FILSAV already exists, DCM3D will print a message to that effect and will stop running. In order to run the problem, either the name of the save file or FILSAV must be changed. This check is designed to prevent accidental destruction of a save file that may be used in a later restart run. The save file is written as an unformatted file and can only be read by DCM $3 D$.

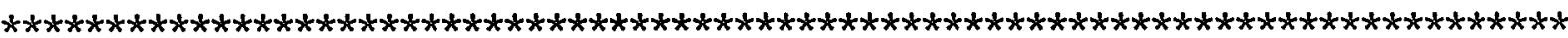

Group E: Options Record

Subgroup E1:

Variables:

Format:

Description:
Options

OPTION(1), OPTION(2)

$2 A 10$

OPTION(1) tells the code to actually run the problem. Set OPTION(1) to RUN if the code is to run the problem after reading the input data. If OPTION(1) is set to anything other than RUN, DCM3D prints out the input data only and stops. Setting OPTION(1) to anything other than RUN is recommended until the input data are correct. If OPTION(1) is set to blank, only a data input check will be made.

OPTION(2) tells the code which type of medium is being solved for. Set OPTION(2) to either SINGLE for a porous medium only run, or DOUBLE for a fractured, porous medium problem. Setting OPTION(2) to anything other than SINGLE, DOUBLE, or blank will cause DCM3D to print a message and stop running. If OPTION(2) is blank, DCM3D will set it to SINGLE. 
Subgroup F1:

Variables:

Format:

Description:
Water Data

DENS, VISC, COMP

3 F10.0

DENS is the density of water. If DENS is set to 0.0 or

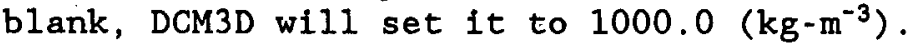

VISC is the dynamic viscosity of water. If VISC is set to 0.0 or blank, DCM3D will set it to 0.001 ( $\mathrm{Pa}-\mathrm{s}$ ).

COMP is the coefficient of bulk compressibility of water. If COMP is set to 0.0 or blank, DCM3D will set it to $4.3 \times 10^{-10}\left(\mathrm{~Pa}^{-1}\right)$.

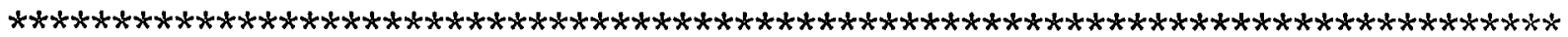

\section{Group G: Gravity Record}

Subgroup G1: Variables:

Format:

Description:
Gravity Description

GRAV, GRAVX, GRAVY, GRAVZ

4F10.0

GRAV is the gravitational coefficient. If GRAV is set to 0.0 or blank, DCM3D will set it to $9.80665\left(\mathrm{~m}^{-\mathrm{s}^{-2}}\right)$.

GRAVX is the $\mathrm{x}$-direction component of gravity. If GRAVX is set to 0.0 or blank, DCM $3 D$ will set it to 0.0

(dimensionless).

GRAVY is the $y$-direction component of gravity. If GRAVY is set to 0.0 or blank, DCM3D will set it to 0.0 (dimensionless).

GRAVZ is the $z$-direction component of gravity. If GRAVZ is set to 0.0 or blank, DCM3D will set it to 0.0 (dimensionless).

GRAVX, GRAVY, and GRAVZ form the gravitational vector and are dimensionless. The code normalizes them to 1.0 after they are read and then uses GRAV to set the correct gravitational vector. GRAVX, GRAVY, and GRAVZ are positive if they point toward the origin from the positive direction. For instance, if the $z$-axis points upwards, GRAVZ is positive if it points downward or toward the origin from the positive direction. See Figure 5-1 for an example.
Subgroup H1: Variables:

Format:
Convergence Criteria

RTOL, ATOL, EPSLON

3F10.0 


\section{Gravitational Term}

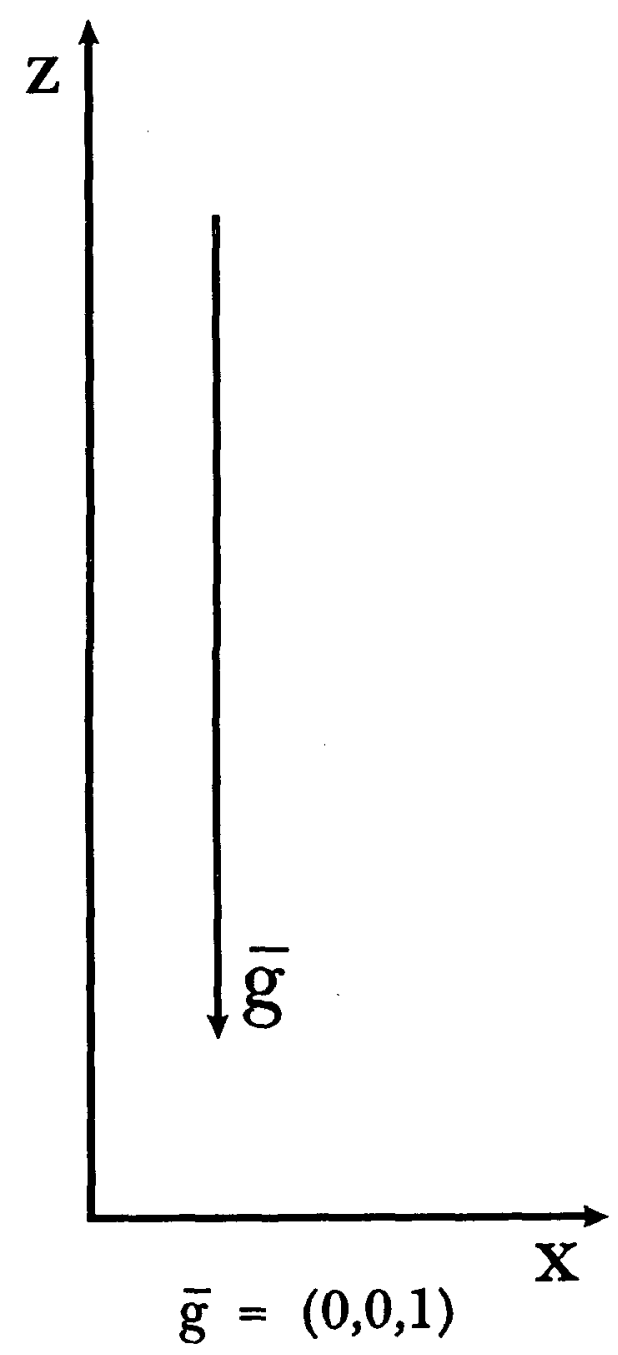

Example of Gravity Vector Pointing to the Origin

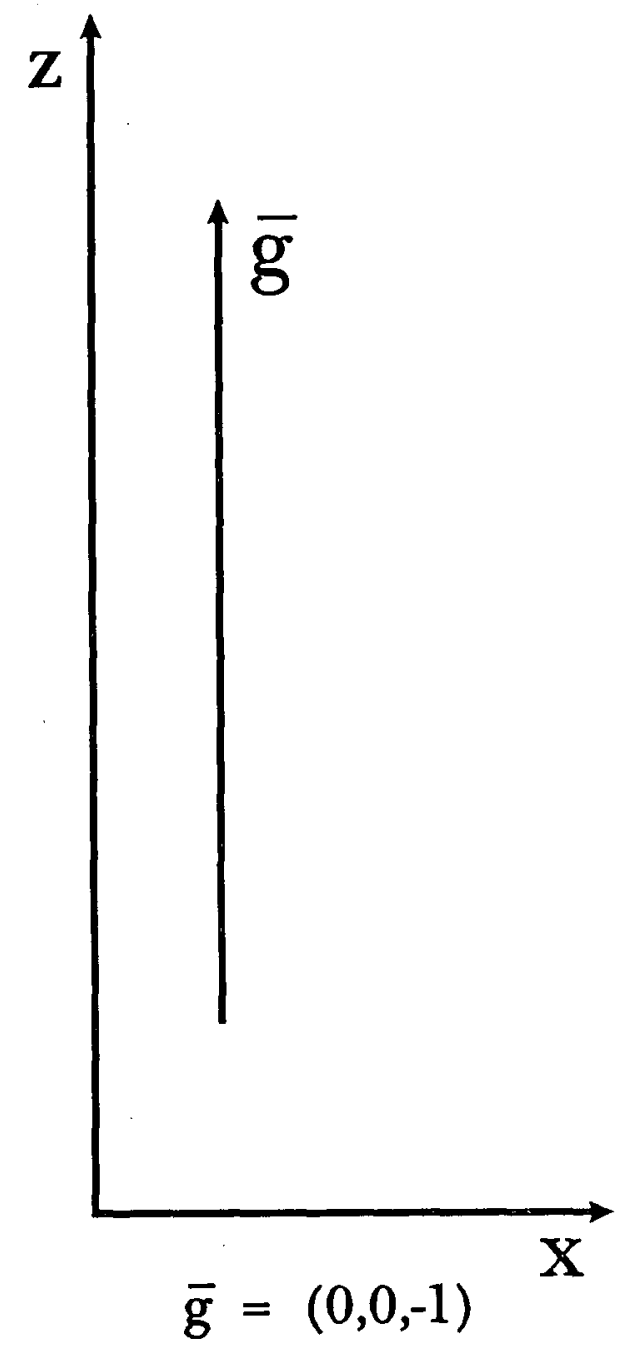

Example of Gravity Vector Pointing away from the Origin

Figure 5-1: Example of Gravity Description 
ATOL is an absolute convergence criteria for LSODES. If ATOL is set to 0.0 or blank, DCM3D will set it to $1.0 \times 10^{-2},(\mathrm{~Pa})$.

EPSLON is the Newton-Raphson convergence criteria. EPSLON is used while determining the interface pressures when the interface permeabilities between two grid blocks are calculated. If EPSLON is set to 0.0 or blank, DCM 3D will set it to $1.0 \times 10^{-5}$ (dimensionless).

LSODES uses RTOL*ABS $(p)+A T O L$, where $p$ is pressure, as its convergence criteria. If the local error is less than the convergence criteria for all grid blocks, then the solution is converged. The LSODES documentation recommends choosing RTOL and ATOL conservatively. However, ATOL may have to be selected based on the units of pressure used in the model. ATOL may have to be smaller if the problem uses pounds per square inch as a pressure unit instead of pascal.

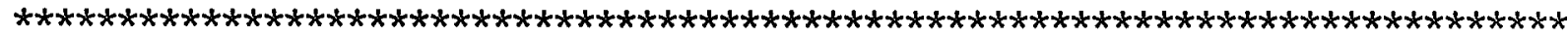

Group I: Time Step Data Record

Subgroup I1: Variables: Format: Description:
Time Step Data

MAXIT, MAXSTP, TSTART, DT, DTMIN, DTMAX, CPUMAX 2 I 10,5 F 10.0

MAXIT is the maximum number of time steps to take to solve the problem. This is set if the user wants to limit the number of time steps for the problem. To be sure there are enough time steps to solve a problem, set MAXIT to a large number. If the MAXIT is set to either 0 or blank, DCM3D will set it to 1000 .

MAXSTP is the maximum number of time steps between write times (see Group $\mathrm{K}$ below). If MAXSTP is set to either 0 or blank, DCM3D will set it to 500. If there is a large time interval between write times, the user may want to set this to a larger number.

TSTART is the start time for the simulation. This is generally set to 0.0 . If TSTART is set to blank, DCM3D will set it to $0.0(\mathrm{~s})$.

DT is the initial time-step size used to start the simulation. Subroutine LSODES usually determines the size of DT itself. It is generally better to set DT to 0.0 and let LSODES pick the initial time-step size. If DT is set too large by the user, convergence problems may 
result. If DT is set to either 0.0 or blank, DCM3D will set it to $0.0(s)$.

DTMIN is the minimum time-step size to take between write times. Subroutine LSODES selects the optimal time-step size to take between write times. If the user sets a value of DTMIN that is too large, convergence problems may result. It is recommended that DTMIN be set to 0.0 . If DTMIN is set to blank, DCM3D will set it to $0.0(\mathrm{~s})$.

DTMAX is the maximum time-step size to take between write times. Subroutine LSODES selects the optimal time-step size to take between write times. The user can set DTMAX if it is desired for LSODES to take a smaller time step. It is recommended that DTMAX be set to 0.0 . If DTMAX is to blank, DCM3D will set it to 0.0 (s).

CPUMAX is the maximum CPU time that the user wants the problem to use. CPU time is checked only between write times. Therefore, the actual time used by the problem may be larger than that actually desired by the user. Because the CPU time is checked only between write times, CPUMAX should be somewhat less than the time limit set for the job. If CPUMAX is set to blank, DCM3D will set it to 60.0 (computer units, usually seconds).

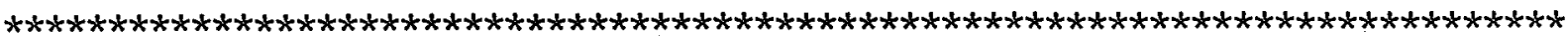

Group J: Grid Block Data Records

Subgroup J1:

Variables:

Format:

Description:
Grid Block Numbers

NXX, NYY, NZZ

$3 \mathrm{I} 10$

NXX is the number of grid blocks in the $x$-direction. If NXX is set to blank, DCM3D will set it to 0 .

NYY is the number of grid blocks in the y-direction. If NYY is set to blank, DCM3D will set it to 0 .

$\underline{N Z Z}$ is the number of grid blocks in the $z$-direction. If $\mathrm{NZZ}$ is set to blank, DCM3D will set it to 0 .

NXX, NYY, or NZZ can be set to 0 . This implies that there are no grid blocks in the direction specified. Setting one of these values to 0 implies that a twodimensional problem is being run. Setting two values to 0 implies that a one-dimensional problem is being run. The code has logic to handle this situation. Setting all three values to 0 will cause DCM3D to print a message and stop running.

Subgroup J2:

$\mathrm{X}$-Direction Grid Block Spacing $\operatorname{DX}(i), \quad i=1, \operatorname{NXX}$

$8 \mathrm{E} 10.0$

Format: 
Description:

Subgroup I3: Variables :

Format:

Description:

Subgroup I4: Variables:

Format:

Description:
DX(i) is the grid block spacing for the $i^{\text {th }}$ grid block in the $\mathrm{x}$-direction. If $\mathrm{NXX}$ is not set to 0 , INT $(\mathrm{NXX} / 8)+1$ lines of grid block data are necessary to specify the $x$ direction grid block spacing. If any of the DX(i)'s are set to blank, DCM3D will set them to 0.0 (m). DCM3D will check all the DX(i)'s for 0.0 values. If any are 0.0 , DCM3D will print a message and stop running. If NXX is set to 0 , the $x$-direction grid block spacing is not included here.

Y-Direction Grid Block Spacing

DY(i) , $i=1, N Y Y$

$8 \mathrm{E} 10.0$

DY(i) is the grid block spacing for the $i^{\text {th }}$ grid block in the $y$-direction. If NYY is not set to $0, \operatorname{INT}(N Y Y / 8)+1$ lines of grid block data are necessary to specify the $y$ direction grid block spacing. If any of the DY(i)'s are set to blank, DCM3D will set them to $0.0(\mathrm{~m})$. DCM3D will check all the DY(i)'s for 0.0 values. If any are 0.0 , DCM3D will print a message and stop running. If NYY is set to 0 , the $y$-direction grid block spacing is not included here.

\section{Z-Direction Grid Block Spacing} $\mathrm{DZ}(\mathrm{i}), \quad i=1, \mathrm{NZZ}$

$8 \mathrm{E} 10.0$

$\mathrm{DZ}(1)$ is the grid block spacing for the $i^{\text {th }}$ grid block in the $z$-direction. If $\mathrm{NZZ}$ is not set to zero, INT(NZZ/8+ 1) lines of grid block data are necessary to specify the $z$-direction grid block spacing. If any of the $D Z(i) ' s$ are set to blank, DCM3D will set them to 0.0 (m). DCM3D will check all the $D Z(i)$ 's for 0.0 values. If any are 0.0 , DCM3D will print a message and stop running. If NZZ is set to 0 , the $z$-direction grid block spacing is not included here.

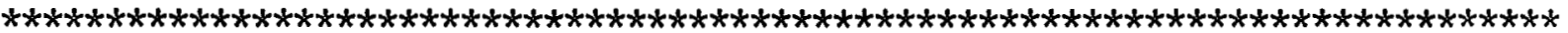

\section{Group K: Write Times Records}

Subgroup K1: Variables:

Format:

Description:
Write Time Number NWRITE

I10.

NWRITE tells the code the number of write times for the problem being solved. A write is considered as a print to the output file, a write to the plot file, or a write to the save file. If a print to the output file and a write to a file are done at the same write time, only one write time is counted. If NWRITE is set to blank, DCM $3 D$ will set it to 0 . DCM3D will test NWRITE for a 0 value. If NWRITE is set to 0 , DCM3D will print a message and stop running. 
Subgroup K2:

Variables:

Format:

Description:
Write Time Data

TWRITE, APRINT, APLOT, ASAVE, AMOIST, AVEL

E10.0, 10X, 5A10

TWRITE is the time at which output in the form of printing to an output file, writing to the plot file, or writing to the save file is desired. TWRITE also tells Subroutine LSODES the times for which solutions are desired by the analyst. In other words, Subroutine LSODES is given a time at which output is desired and then it solves the difference equations to that time. If TWRITE is set to blank, DCM3D will set it to $0.0(\mathrm{~s})$. If TWRITE is less than or equal to TSTART on the TIME STEP DATA record (Group I), DCM3D will print a message and stop running. In addition, if a TWRITE time is less than or equal to a preceding TWRITE time, DCM3D will print a message and stop running.

APRINT tells DCM3D whether or not a print to the output file is desired at this write time. If APRINT is set to PRINT, the output is printed to the output file. If APRINT is set to anything else or to blank, nothing will be printed to the output file.

APLOT tells DCM3D whether or not a write to the plot file is desired for this write time. If APLOT is set to PLOT, the output is written to the plot file. If APLOT is set to anything else or to blank, nothing will be written to the PLOT file. If APLOT is set to PLOT, but no plot file is specified on the FILES record (i.e. FILPLT is set to blank in Group D), DCM3D will print a message and stop running. The plot file structure is presented in Section 5.6 .

ASAVE te11s DCM3D whether or not to save the input and output for this write time. If ASAVE is set to SAVE, the input data and output data are written to the save file. If ASAVE is set to anything else or to blank, nothing will be written to the save file. It is recommended that ASAVE be set to SAVE occasionally during a run if there is danger that CPU time or maximum iterations may be exceeded. If ASAVE is set to SAVE, but no save file is specified on the FILES record (i.e. FILSAV is set to blank in Group D), DCM3D will print a message and stop running.

AMOIST tells DCM3D whether or not to calculate either the moisture content or saturations for this write time. If AMOIST is set to MOISTURE, the moisture content for the porous medium and fractures (for a fractured porous medium run) is calculated. If AMOIST is set to SATURATION, the degree of saturation is calculated. If AMOIST is set to anything else or to blank, neither moisture contents nor saturations are calculated. If APRINT is set to PRINT and/or APLOT is set to PLOT, the 
moisture contents or saturations are written to the respective output file and plot file.

AVEL tells the code whether or not to calculate the Darcy velocities for this write time. If AVEL is set to VELOCITY, the Darcy velocities are calculated. If AVEL is set to anything else or to blank, the Darcy velocities are not calculated. If APRINT is set to PRINT and/or APLOT is set to PLOT, the Darcy velocities are written to the respective output file and plot file.

Subgroup K2 is provided NWRITE times.

The last write time, TWRITE(NWRITE), is the ending time for the simulation.

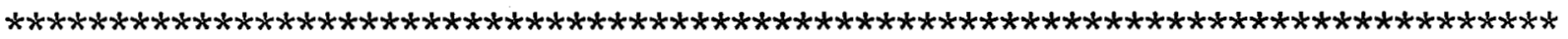

\section{Group L: Material Data Records}

Subgroup L1: Variables:

Format:

Description:

Subgroup L2:

Variables:

Format:

Description:
Material Numbers NUMMAT

I10

NUMMAT tells the code the number of materials in the modeled region. There must be at least one material for any problem being run. If NUMMAT is set to blank, DCM3D will set it to 0 . If NUMMAT is less than or equal to 0 , DCM3D will print a message and stop running.

Material Type and Ranges

MATNAM, IMIN, IMAX, JMIN, JMAX, KMIN, KMAX

A5, 5X, 6I5

MATNAM is the name of the particular material or soil type for which hydrologic information is being provided. MATNAM can be any five-letter alphanumeric name except UNDEF. The code uses UNDEF to check for grid blocks that have no material assignments. If a material name is set to UNDEF, the DCM3D will print a message and stop running.

IMIN is the minimum $i$-index range for the particular material.

IMAX is the maximum $i$-index range for the particular material.

JMIN is the minimum $\mathbf{j}$-index range for the particular material.

JMAX is the maximum $\mathrm{j}$-index range for the particular index.

KMIN is the minimum $k$-index range for the particular material. 
Subgroup L3: Variables:

Format:

Description:
KMAX is the maximum $\mathrm{k}$-index range for the particular material.

IMIN and IMAX must be between 1 and NXX. If NXX is 0 , then IMIN and IMAX must be set to 1 . JMIN and JMAX must be between 1 and NYY. If NYY is set to 0 , then JMIN and JMAX must be set to 1 . KMIN and KMAX must be between 1 and NZZ. If NZZ is set to 0 , then KMIN and KMAX must be set to 1 .

A read of this record will cause an overwrite a previous read of this record. For instance, suppose material 2 occupies only a quadrant of the modeled grid. The remainder of the modeled region consists of material 1 . The minimum/maximum range for material 1 , which is read first, could list the entire range of grid blocks of the modeled region. The minimum/maximum range for material 2 , which is read second, would just list the minimum/maximum ranges necessary to change the appropriate grid blocks to material 2 .

Porous Medium Hydrologic Property Record COMPR, POROS, PERXX, PERYY, PERZZ

$5 \mathrm{E} 10.0$

COMPR is the porous medium bulk modulus of compressibility of the material. If COMPR is set to blank, DCM 3D sets it to $0.0\left(\mathrm{~Pa}^{-1}\right)$.

POROS is the porous medium porosity of the material. If POROS is set to blank, DCM3D sets it to 0.0 (dimensionless).

PERXX is the porous medium intrinsic permeability of the material in the $x$-direction. If PERXX is set to blank, DCM3D sets it to $0.0\left(\mathrm{~m}^{2}\right)$.

PERYY is the porous medium intrinsic permeability of the material in the y-direction. If PERYY is set to blank, DCM3D sets it to $0.0\left(\mathrm{~m}^{2}\right)$.

PERZZ is the porous medium intrinsic permeability of the material in the $z$-direction. If PERZZ is set to blank, DCM $3 D$ sets it to $0.0\left(\mathrm{~m}^{2}\right)$.

Subgroup L4:

Porous Medium Saturation Property Record

Variables:

Format:

Description:
SS, SR, N, M, ALPHA

$5 F 10.0$

$\underline{\text { SS }}$ is the porous medium saturation under fully saturated conditions for the van Genuchten equations for capillary pressure and relative permeability. If this parameter is blank or set to 0.0 , DCM $3 D$ sets it to 1.0

(dimensionless). 
$S R$ is the porous medium residual saturation for the van Genuchten equations. If $S R$ is set to blank, DCM3D sets it to 0.0 (dimensionless).

$N$ is the power of the denominator in the van Genuchten equation for capillary pressure. If $N$ is set to 0.0 or blank, DCM3D sets it to $1.0 /(1.0-\mathrm{M}$ ) (dimensionless).

$M$ is the power of the whole expression of the van Genuchten capillary equation. If $M$ is set to 0.0 or blank, DCM3D sets it to $1.0-1.0 / \mathrm{N}$ (dimensionless). If both $\mathrm{N}$ and $\mathrm{M}$ are zero and/or blank, DCM3D will print a message and stop running.

ALPHA is a parameter in the denominator of the van Genuchten equation for capillary pressure. If it is set to blank, DCM3D sets it to $0.0\left(\mathrm{~Pa}^{-1}\right)$.

For purposes of the data input, the van Genuchten capillary pressure-saturation relationship is defined by:

$$
S=\left(S_{\mathrm{s}}-S_{\mathrm{r}}\right)\left(\frac{1}{1+\left(\alpha p_{\mathrm{c}}\right)^{\mathrm{N}}}\right)^{\mathrm{M}}+S_{\mathrm{r}}
$$

Subgroup L5: Variables: Format: Description:
Fracture Hydrologic Property Record COMPR, POROS, PERXX, PERYY, PERZZ, AVFL $6 \mathrm{E} 10.0$

COMPR is the fracture bulk modulus of compressibility of the material. If COMPR is set to blank, DCM $3 D$ sets it to $0.0\left(\mathrm{~Pa}^{-1}\right)$.

POROS is the fracture porosity of the material. If POROS is set to blank, DCM3D sets it to 0.0 (dimensionless).

PERXX is the fracture intrinsic permeability of the material in the $\mathrm{x}$-direction. If PERXX is set to blank, DCM3D sets it to $0: 0\left(\mathrm{~m}^{2}\right)$.

PERYY is the fracture intrinsic permeability of the material in the y-direction. If PERYY is set to blank, DCM3D sets it to $0.0\left(\mathrm{~m}^{2}\right)$.

PERZZ is the fracture intrinsic permeability of the material in the $z$-direction. If PERZZ is set to blank, DCM3D sets it to $0.0 .\left(\mathrm{m}^{2}\right)$.

AVFL is the transfer factor between the porous medium and the fractures. It is defined by Eq. $2-19$. This parameter is only applicable if OPTION(2) is set to DOUBLE. If OPTION(2) is set to SINGLE, DCM3D will set 
AVFL to 0.0. If AVFL is set to blank, DCM3D will set it to 0.0 (dimensionless).

Subgroup L6:

Variables:

Format:

Description:
Fracture Saturation Property Record

SS, SR, N, M, ALPHA

5 F10.0

$\underline{\text { SS }}$ is the fracture saturation under fully saturated conditions for the van Genuchten equations for capillary pressure and relative permeability. If $S S$ is blank or set to 0.0 , DCM $3 D$ sets it to 1.0 (dimensionless).

SR is the fracture residual saturation for the van Genuchten equations: If SR is set to blank, DCM3D sets it to 0.0 (dimensionless).

$\underline{N}$ is the power of the denominator in the van Genuchten equation for capillary pressure. If $\mathrm{N}$ is set to 0.0 or blank, DCM 3D sets it to $1.0 /(1.0-M)$ (dimensionless).

$\underline{M}$ is the power of the whole expression of the van Genuchten capillary equation. If $M$ is set to 0.0 or blank, DCM $3 D$ sets it to $1.0-1.0 / \mathrm{N}$ (dimensionless). If both $N$ and $M$ are 0.0 and/or blank, DCM3D prints a message and stops running.

ALPHA is a parameter in the denominator of the van Genuchten equation for capillary pressure. If ALPHA is set to blank, DCM3D sets it to $0.0\left(\mathrm{~Pa}^{-1}\right)$.

See Eq. 5-1 for the use of SS, SR, M, N, and ALPHA.

Subgroups L5 and L6 are required only if OPTION(2) is set to DOUBLE. If OPTION(2) is set to SINGLE and Subgroups L5 and L6 are included, a read error will probably occur later while reading the input file.

Subgroups L2, L3, and L4 (and L5 and L6 if OPTION(2) is set to DOUBLE) are required for each material. There should be NUMMAT sets of Subgroup L2 through Subgroup L6.

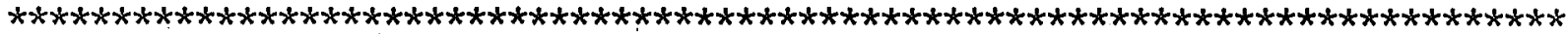

Group M: Pressure Initial Conditions Records

Subgroup M1: Variables:

Format:

Description:
Porous Medium Pressure Initial Conditions $\operatorname{PRESS}(n), n=1, N X * N Y * N Z$ where $\mathrm{NX}=\mathrm{MAX}(1, \mathrm{NXX}), \mathrm{NY}=\mathrm{MAX}(1, \mathrm{NYY})$, and $\mathrm{NZ}=\mathrm{MAX}(1, \mathrm{NZZ})$ $8 \mathrm{E} 10.0$

PRESS $(n)$ is the initial porous medium pressure in the $n^{\text {th }}$ grid block. The pressure is negative in the unsaturated zone and positive in the saturated zone. If any pressures are set to blank, DCM3D will set them to 0.0 $(\mathrm{Pa})$. The porous medium pressures are read in the following manner. 
The pressures are read in the $x$-direction for $j=1$ and $k=1$ until all pressures for that row of grid blocks are read. $\operatorname{INT}(\mathrm{NXX} / 8)+1$ records are required to read one row of grid block pressures. The $\mathbf{j}$-index is then incremented and pressures for the new row are then read. The process is repeated until $j=\mathrm{NY}$. Then, the $k$-index is incremented by one, $j$ is set to one, and the process repeated. For example, the pressure input should look like the following for $\mathrm{NX}=10, \mathrm{NY}=3$, and $\mathrm{NZ}=2$ :

\begin{tabular}{|c|c|c|c|c|c|c|}
\hline$p(1)$ & $p(2)$ & $p(3)$ & $p(4)$ & $p(5)$ & $p(6) \quad p(7)$ & $\mathrm{p}(8)$ \\
\hline $\mathrm{p}(9)$ & $p(10)$ & & $(i=1$ & to $\mathrm{NX}$, & $\mathrm{j}=1, \quad \mathrm{k}=1)$ & \\
\hline$p(11)$ & $\mathrm{p}(12)$ & $p(13)$ & $p(14)$ & $p(15)$ & $p(16) \quad p(17)$ & $\mathrm{p}(18)$ \\
\hline$p(19)$ & $p(20)$ & & $(i=1$ & to $\mathrm{NX}$, & $j=2, \quad k=1)$ & \\
\hline$p(21)$ & $p(22)$ & $p(23)$ & $p(24)$ & $p(25)$ & $p(26) \quad p(27)$ & $\mathrm{p}(28)$ \\
\hline $\mathrm{p}(29)$ & $p(30)$ & & $(i=1$ & to $\mathrm{NX}$, & $j=3, \quad k=1)$ & \\
\hline$p(31)$ & $p(32)$ & $p(33)$ & $p(34)$ & $p(35)$ & $p(36) \quad p(37)$ & $p(38)$ \\
\hline$p(39)$ & $p(40)$ & & $(i=1$ & to $\mathrm{NX}$, & $j=1, \quad k=2)$ & \\
\hline$p(41)$ & $p(42)$ & $p(43)$ & $p(44)$ & $\mathrm{p}(45)$ & $p(46) \quad p(47)$ & $p(48)$ \\
\hline$p(49)$ & $p(50)$ & & $(i=1$ & to $\mathrm{NX}$, & $j=2, \quad k=2)$ & \\
\hline $\mathrm{p}(51)$ & $p(52)$ & $p(53)$ & $p(54)$ & $p(55)$ & $p(56) \quad p(57)$ & $p(58)$ \\
\hline$p(59)$ & $p(60)$ & & $(i=1$ & to $\mathrm{NX}$, & $\mathrm{j}=3, \quad \mathrm{k}=2)$ & \\
\hline
\end{tabular}

Subgroup M2: Variables: Format: Description:
Subgroup M1 must be included for the problem being run.

Fracture Pressure Initial Conditions

$\operatorname{PRESS}(\mathrm{n}), \mathrm{n}=\mathrm{NX} * \mathrm{NY} * \mathrm{NZ}+1, \quad 2 * \mathrm{NX} * \mathrm{NY} * \mathrm{NZ}$

$8 \mathrm{E} 10.0$

PRESS $(n)$ is the initial fracture pressure in a grid block. The pressure is negative in the unsaturated zone and positive in the saturated zone. The fracture pressures are read in the same manner as above. The pressures are read in the $x$-direction for $j=1$ and $k=1$ until all pressures for that row of grid blocks are read in. INT $(\mathrm{NXX} / 8)+1$ records are required to read one row of grid block pressures. $j$ is then incremented and pressures for the new row are then read. The process is repeated until $j=N Y$. Then, $k$ is incremented by one, $j$ is set to one, and the process repeated. For example, if the preceding example from the porous medium pressure is continued, the fracture pressure input should look like:

$$
\begin{array}{rlllllll}
p(61) & p(62) & p(63) & p(64) & p(65) & p(66) & p(67) & p(68) \\
p(69) & p(70) & & (i=1 \text { to } N X, j=1, k=1) & \\
p(71) & p(72) & p(73) & p(74) & p(75) & p(76) & p(77) & p(78) \\
p(79) & p(80) & & (i=1 \text { to } N X, j=2, k=1) & \\
p(81) & p(82) & p(83) & p(84) & p(85) & p(86) & p(87) & p(88) \\
p(89) & p(90) & & (i=1 \text { to } N X, j=3, k=1) & \\
p(91) & p(92) & p(93) & p(94) & p(95) & p(96) & p(97) & p(98) \\
p(99) & p(100) & & (i=1 \text { to } N X, j=1, k=2) & \\
p(101) & p(102) & p(103) & p(104) & p(105) & p(106) & p(107) & p(108) \\
p(109) & p(110) & & (i=1 \text { to NX, j=2, } k=2) & \\
p(111) & p(112) & p(113) & p(114) & p(115) & p(116) & p(117) & p(118) \\
p(119) & p(120) & & (i=1 \text { to NX, } j=3, k=2) &
\end{array}
$$


Group N: X-Direction Boundary Condition Data

(If NXX is 0 or blank, this group is not provided)

Subgroup N1:

Variables:

Format:

Description:

Subgroup N2:

Variables:

Format:

Description:
Boundary Condition Numbers for X-Direction, Left Face $\mathrm{NBC}(1)$

$\mathrm{I} 10$

NBC(1) is the total number of non-zero flux and pressure boundary condition tables on the left face of the boundary pointing in the $x$-direction. If the entire boundary is zero flux, then set $\operatorname{NBC}(1)$ to 0 . Otherwise, set $\mathrm{NBC}(1)$ to the number of boundary conditions applied to the side. If $\mathrm{NBC}(1)$ is set to 0 , Subgroups $\mathrm{N} 2$ to $\mathrm{N} 4$ are not read and should not be included in the input file.

Boundary Condition Type

NTIME, BCTYPM, BCTYPF, BCMTH

I10, 3AI0

NTIME is the number of data pairs or triplets in the table of boundary condition data for the left face pointing in the $x$-direction (see Subgroup N4).

BCTYPM is the type of porous medium boundary condition applied to the left face pointing in the $\mathrm{x}$-direction. The choice is either FLUX if the boundary condition is a flux boundary condition (in terms of volume/time/unitarea or velocity) or PRESSURE if the boundary condition is a specified pressure.

BCTYPF is the type of fracture boundary condition applied to the left face pointing in the $\mathrm{x}$-direction. The choice is either FLUX if the boundary condition is a flux boundary condition (in terms of volume/time/unit-area or velocity) or PRESSURE if the boundary condition is a pressure. BCTYPF is required only if OPTION(2) is set to DOUBLE.

BCMTH tells the code how to distribute the boundary conditions between the porous medium and fractures on the left face pointing in the $x$-direction. It is only applicable if BCTYPM and BCTYPF are the same and OPTION(2) is set to DOUBLE.

If BCTYPM and BCTYPF are both set to FLUX, the choice for BCMTH is either PRESCRIBED or MOBILITY. PRESCRIBED is set if the boundary fluxes are described by the user and provided in the table below (see Subgroup N4). MOBILITY is set if the fluxes are distributed between the porous 
Subgroup N3:

Variables:

Format:

Description:
Subgroup N4:

Variables:

Format:

Description: medium and fractures based on the mobility coefficient of the two.

If BCTYPM and BCTYPF are both set to PRESSURE, the choice for BCMTH is either PRESCRIBED or SAME. PRESCRIBED is set if the boundary fluxes are described by the user and provided in the table below (see Subgroup N4). SAME is set if boundary pressures for both the porous medium and fractures are the same. Such a condition may occur if both the porous medium and the fractures are saturated on that boundary.

If BCTYPM is different than BCTYPF, then BCMTH is not specified.

A flowchart for defining BCTYPM, BCTYPF, and BCMTH is presented in Figure 5-2.

Boundary Condition Range

JMIN, JMAX, KMIN, KMAX

$4 \mathrm{I} 10$

JMIN is the minimum index in the $y$-direction of the boundary condition applied to the left face pointing in the $\mathrm{x}$-direction.

JMAX is the maximum index in the $y$-direction of the boundary condition applied to the left face pointing in the $\mathrm{x}$-direction.

KMIN is the minimum index in the $z$-direction of the boundary condition applied to the left face pointing in the $\mathrm{x}$-direction.

KMAX is the maximum index in the $z$-direction of the boundary condition applied to the left face pointing in the $\mathrm{x}$-direction.

JMIN and JMAX must be between 1 and NYY. If NYY is 0 , set JMIN and JMAX to 1. KMIN and KMAX must be between 1 and NZZ. If NZZ is 0 , set KMIN and KMAX to 1 .

Boundary Condition Data

BCTIM, BCMAT, BCFRC

3E10.0

BCTIM is the time at which the boundary condition is applied to the left face pointing in the $x$-direction.

BCMAT is, under most conditions, the value of the porous medium boundary condition applied to the left face pointing in the $x$-direction at the time, BCTIM.

BCFRC is the value of the fracture boundary condition applied to the left face pointing in the $x$-direction at the time, BCTIM. 


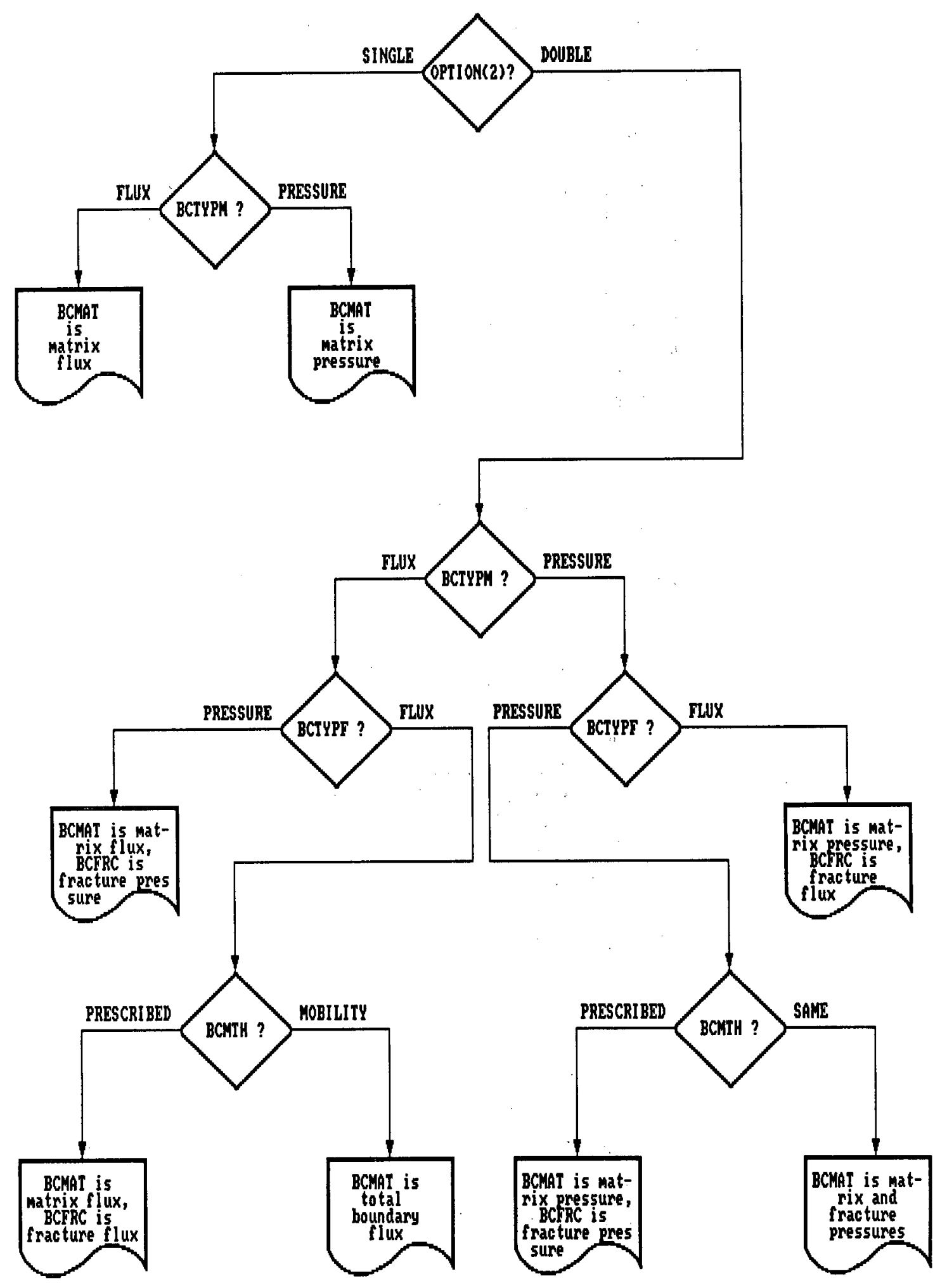

Figure 5-2: Flow Chart for Selecting Boundary Conditions 
If OPTION(2) is set to SINGLE, then the following rules for the selection of BCMAT values apply.

1. If BCTYPM is set to FLUX, then BCMAT is set to the porous medium flux value $\left(\mathrm{m}^{3} / \mathrm{s} / \mathrm{m}^{2}\right)$. If the flux is into the porous medium, then BCMAT is set to a positive value. If the flux is out from the porous medium, then BCMAT is set to a negative value.

2. If BCTYPM is set to PRESSURE, then BCMAT is set to porous medium pressure value $(\mathrm{Pa})$. If the boundary condition is unsaturated, then a negative pressure is specified. If the boundary condition is saturated, then a positive pressure is specified.

3. BCFRC values are not specified.

If OPTION(2) is set to DOUBLE, then the following rules for the selection of BCMAT and BCFRC values apply.

1. If BCTYPM is set to FLUX and BCTYPF is set to PRESSURE, then BCMAT is a porous medium flux value and BCFRC is a fracture pressure value.

2. If BCTYPM is set to PRESSURE and BCTYPF is set to FLUX, then BCMAT is a porous medium pressure value and BCFRC is a fracture flux value.

3. If BCTYPM and BCTYPF are both set to FLUX and BCMTH is set to PRESCRIBED, then BCMAT is set to a porous medium flux value and BCFRC is set to a fracture flux value.

4. If BCTYPM and BCTYPF are both set to FLUX and BCMTH is set to MOBILITY, then BCMAT is set to the total porous medium and fracture flux. BCFRC is left blank. DCM 3D uses the BCMAT values and mobility coefficients to allocate fluxes between the porous medium and the fractures.

5. If BCTYPM and BCTYPF are both set to PRESSURE and BCMTH is set to PRESCRIBED, then BCMAT is set to a porous medium pressure value and BCFRC is set to a fracture pressure value.

6. If BCTYPM and BCTYPF are both set to PRESSURE and BCMTH is set to SAME, then BCMAT is set to the pressure value for both porous medium and fracture. BCFRC is left blank. DCM3D sets the BCFRC values to BCMAT values.

Figure 5-2 will aid in setting values for BCMAT and BCFRC. 
Subgroup $\mathrm{N} 4$ is provided NTIME (Subgroup N2) times. Figure 5-3 shows how the boundary condition data are read.

This Subgroup N4 specifies a table of boundary conditions for which the following rules apply.

1. If simulation time, $t$, is less than $\operatorname{BCTIM(1),~then~}$ the boundary condition values are set to 0.0 .

2. If simulation time is between BCTIM(i) and $\operatorname{BCTIM}(i+1)$, then the boundary conditions are determined from a linear interpolation.

3. If simulation time is larger than BCTIME(NTIME), then the boundary condition values are set to the last value in the table.

Subgroups N2, N3, and $\mathrm{N} 4$ are provided $\mathrm{NBC}(1)$ times. If $\mathrm{NBC}(1)$ is set to 0 , then Subgroups $N 2, N 3$, and $N 4$ are not provided.

Subgroup N5:

Variables:

Boundary Condition Numbers for X-Direction, Right Face

Format: $\mathrm{NBC}(2)$

Description:

Subgroup N6:

Variables:

Format:

Description:

I10

$\operatorname{NBC}(2)$ is the total number of non-zero flux and pressure boundary condition tables on the right face of the boundary pointing in the $x$-direction. The description of $\mathrm{NBC}(2)$ is the same as $\mathrm{NBC}(1)$ under subgroup N1.

Boundary Condition Type

NTIME, BCTYPM, BCTYPF, BCMTH

I10, 3A10

NTIME is the number of data pairs or triplets in the table of boundary condition data for the right face pointing in the $x$-direction (see Subgroup $N 8$ ).

BCTYPM is the type of porous medium boundary condition applied to the right face pointing in the $\mathrm{x}$-direction. The choices are the same as for Subgroup N2.

BCTYPF is the type of fracture boundary condition applied to the right face pointing in the $x$-direction. The choices are the same as for Subgroup N2.

BCMTH tells the code how to distribute the boundary conditions between the porous medium and fractures for the right face pointing in the $\mathrm{x}$-direction. The choice is the same as for Subgroup N2 except that references to Subgroup N4 are to Subgroup N8 instead.

Subgroup N7: Boundary Condition Range

Variables: JMIN, JMAX, KMIN, KMAX

Format:

$4 \mathrm{I} 10$ 


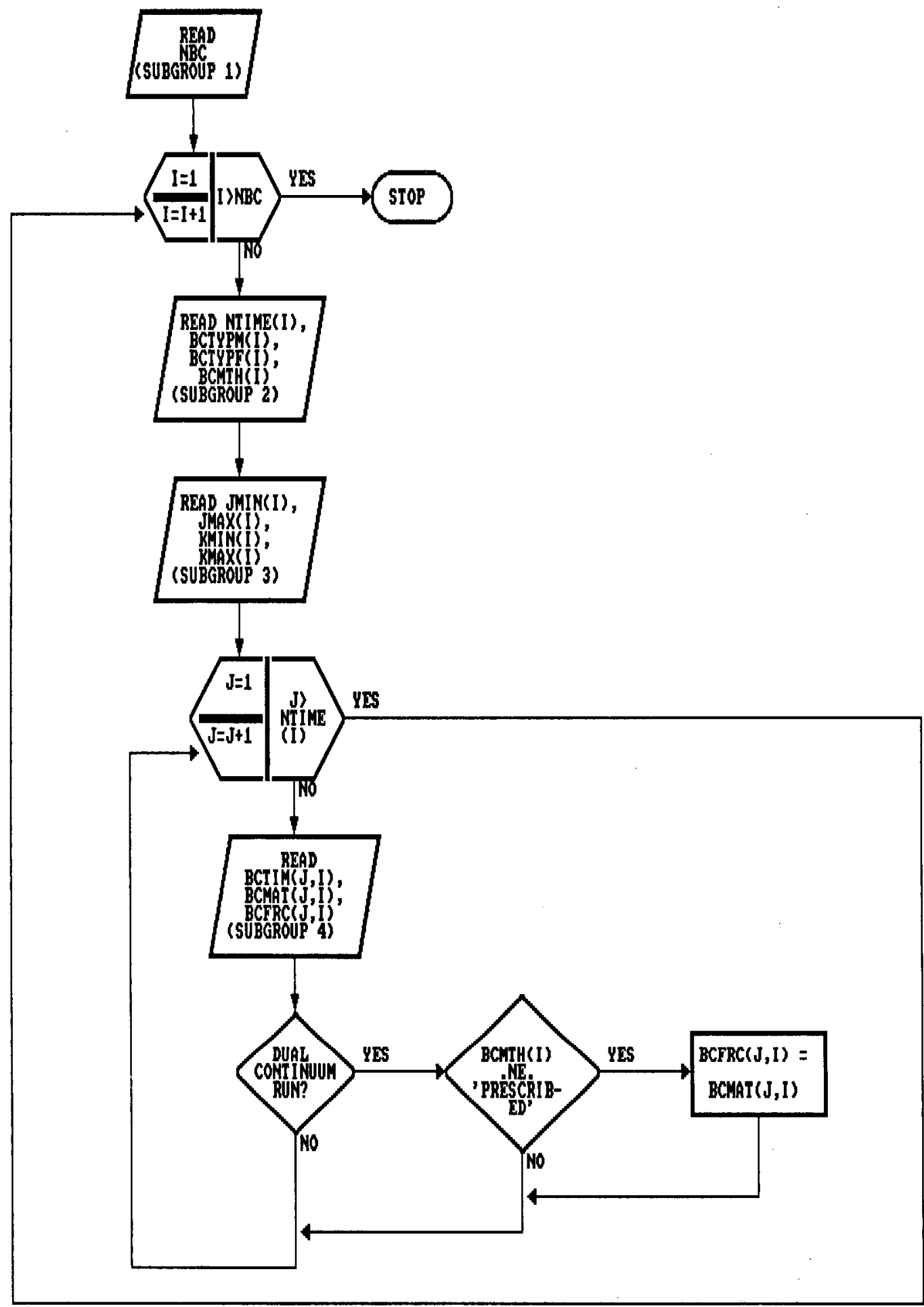

Figure 5-3: Flow Chart for Reading Boundary Conditions 
Description: JMIN is the minimum index in the y-direction of the boundary condition applied to the right face pointing in the $\mathrm{x}$-direction.

JMAX is the maximum index in the y-direction of the boundary condition applied to the right face pointing in the $\mathrm{x}$-direction.

KMIN is the minimum index in the $z$-direction of the boundary condition applied to the right face pointing in the $\mathrm{x}$-direction.

KMAX is the maximum index in the z-direction of the boundary condition applied to the right face pointing in the $\mathrm{x}$-direction.

Conditions on JMIN, JMAX, KMIN, and KMAX are the same as those for subgroup N3.

Subgroup N8:

Boundary Condition Data

Variables:

BCTIM, BCMAT, BCFRC

Format:

$3 \mathrm{E} 10.0$

Description:

BCTIM is the time at which the boundary condition is applied to the right face pointing in the $\mathrm{x}$-direction.

BCMAT is, under most conditions, the value of the porous medium boundary condition applied to the right face pointing in the $\mathrm{x}$-direction at time, BCTIM. The description for BCMAT is the same as that described for Subgroup N4.

BCFRC is the value of the fracture boundary condition applied to the right face pointing in the $\mathrm{x}$-direction at time, BCTIM. The description for BCFRC is the same as that described for Subgroup N4.

The conditions applied to Subgroup $\mathrm{N} 8$ are the same as those applied to Subgroup N4. Subgroup N8 is provided NTIME (Subgroup N6) times.

Rules and choices applicable to Subgroups N2, N3, and N4 are applicable to Subgroups $\mathrm{N} 6, \mathrm{~N} 7$, and $\mathrm{N} 8$, respectively.

Subgroups $\mathrm{N} 6, \mathrm{~N} 7$, and $\mathrm{N} 8$ are provided $\mathrm{NBC}(2)$ times. If $\operatorname{NBC}(2)$ is set to 0 , then Subgroups $N 6, N 7$, and $N 8$ are not provided.

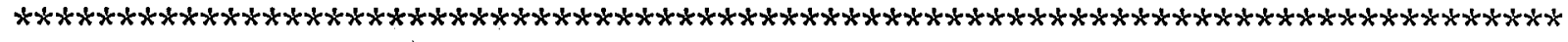

Group 0: Y-Direction Boundary Condition Data (If NYY is 0 or blank, this group is not provided)

Subgroup 01: Boundary Condition Numbers for Y-Direction, Left Face Variables: $\operatorname{NBC}(3)$ 
Format:

Description:

Subgroup 02:

Variables:

Format:

Description:

Subgroup 03:

Variables:

Format:

Description:
I10

NBC(3) is the total number of non-zero flux and pressure boundary condition tables on the left face of the boundary pointing in the y-direction. The description of $\mathrm{NBC}(3)$ is the same as $\mathrm{NBC}(1)$ under Subgroup N1.

Boundary Condition Type

NTIME, BCTYPM, BCTYPF, BCMTH

I10, 3A10

NTIME is the number of data pairs or triplets in the table of boundary condition data for the left face pointing in the $y$-direction (see Subgroup 04).

BCTYPM is the type of porous medium boundary condition applied to the left face pointing in the y-direction. The choices are the same as those for Subgroup N2.

BCTYPF is the type of fracture boundary condition applied to the left face pointing in the y-direction. The choices are the same as those for Subgroup N2.

BCMTH tells the code how to distribute the boundary conditions between the porous medium and fractures for the left face pointing in the y-direction. The choices are the same as those for Subgroup N2 except that references to Subgroup N4 are to Subgroup 04 instead.

Boundary Condition Range

IMIN, IMAX, KMIN, KMAX

$4 \mathrm{I} 10$

IMIN is the minimum index in the $x$-direction of the boundary condition applied to the left face pointing in the $\mathrm{y}$-direction.

IMAX is the maximum index in the $x$-direction of the boundary condition applied to the left face pointing in the $\mathrm{y}$-direction.

KMIN is the minimum index in the z-direction of the boundary condition applied to the left face pointing in the $y$-direction.

KMAX is the maximum index in the $z$-direction of the boundary condition applied to the left face pointing in the $\mathrm{y}$-direction.

IMIN and IMAX must be between 1 and NXX. If $\mathrm{NXX}$ is 0 , set IMIN and IMAX to 1. KMIN and KMAX must be between 1 and NZZ. If NZZ is 0 , set KMIN and KMAX to 1 .

Boundary Condition Data

BCTIM, BCMAT, BCFRC

$3 \mathrm{E} 10.0$ 

applied to the left face pointing in the $y$-direction.

BCMAT is, under most conditions, the value of porous medium boundary condition applied to the left face pointing in the y-direction at the time, BCTIM. The description for BCMAT is the same as that described for Subgroup N4.

BCFRC is the value of the fracture boundary condition applied to the left face pointing in the $y$-direction at the time, BCTIM. The description for BCFRC is the same as that described for Subgroup N4.

The conditions applied to Subgroup 04 are the same as those applied to Subgroup $\mathrm{N4}$. Subgroup 04 is provided NTIME (Subgroup 02) times.

Subgroups 02,03 , and 04 are provided $\mathrm{NBC}(3)$ times. If $\mathrm{NBC}(3)$ is set to 0 , then Subgroups 02,03 , and 04 are not provided.

Subgroup 05:

Variables:

Format:

Description:

Subgroup 06:

Variables:

Format:

Description:
Boundary Condition Numbers for Y-Direction, Right Face $\operatorname{NBC}(4)$

I10

NBC(4) is the number of non-zero flux and pressure boundary condition tables on the right face of the boundary pointing in the y-direction. The description of $\mathrm{NBC}(4)$ is the same as $\mathrm{NBC}(1)$ under Subgroup N1.

Boundary Condition Type

NTIME, BCTYPM, BCTYPF, BCMTH

I10, 3A10

NTIME is the number of data pairs or triplets in the table of boundary condition data for the right face pointing in the y-direction (see Subgroup 08).

BCTYPM is the type of porous medium boundary condition applied to the right face pointing in the y-direction. The choices ar 2 the same as those for Subgroup N2.

BCTYPF is the type of fracture boundary condition applied to the right face in the y-direction. The choices are the same as those for Subgroup N2.

BCMTH tells the code how to distribute the boundary conditions between the porous medium and fractures for the right face pointing in the y-direction. The choices are the same as for Subgroup N2 except that references to Subgroup $\mathrm{N} 4$ are to Subgroup 08 instead.

Boundary Condition Range

IMIN, IMAX, KMIN, KMAX

$4 \mathrm{I} 10$
Variables:

Format: 
IMIN is the minimum index in the $x$-direction of the boundary condition applied to the right face pointing in the $y$-direction.

IMAX is the maximum index in the $x$-direction of the boundary condition applied to the right face pointing in the y-direction.

KMIN is the minimum index in the $z$-direction of the boundary condition applied to the right face pointing in the $y$-direction.

KMAX is the maximum index in the $z$-direction of the boundary condition applied to the right face pointing in the $\mathrm{y}$-direction.

Conditions on IMIN, IMAX, KMIN, and KMAX are the same as those for Subgroup 03.

Subgroup 08 : Variables: Format: Description:

\section{Boundary Condition Data}

BCTIM, BCMAT, BCFRC $3 \mathrm{E} 10.0$

BCTIM is the time at which the boundary condition is applied to the right face pointing in the $y$-direction.

BCMAT is, under most conditions, the value of the porous medium boundary condition applied to the right face pointing in the $y$-direction at time BCTIM. The description for BCMAT is the same as that described for Subgroup N4.

BCFRC is the value of the fracture boundary condition applied to the right face pointing in the y-direction at time BCTIM. The description for BCFRC is the same as that described for Subgroup N4.

The conditions applied to Subgroup 08 are the same as those applied to Subgroup N4. Subgroup 08 is provided NTIME (Subgroup 06) times.

Rules and choices applicable to Subgroups N2, N3, and N4 are applicable to Subgroups 06, 07, and 08, respectively.

Subgroups 06, 07, and 08 are provided $\mathrm{NBC}(4)$ times. If $\mathrm{NBC}(4)$ is set to 0 , then Subgroups 06,07 , and 08 are not provided.

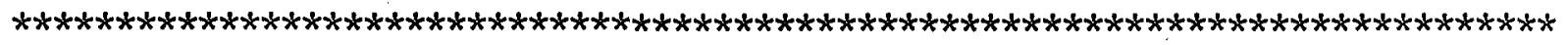

Group P: Z-Direction Boundary Condition Data

(If $\mathrm{NZZ}$ is 0 or blank, this group is not provided)

Subgroup P1: Boundary Condition Numbers for Z-Direction, Left Face Variables: NBC (5) 
Format:

Description:

Subgroup P2:

Variables:

Format:

Description:

Subgroup P3:

Variables:

Format:

Description:
I10

NBC(5) is the total number of non-zero flux and pressure boundary condition tables on the left face of the boundary pointing in the $z$-direction. The description of $\mathrm{NBC}(5)$ is the same as $\mathrm{NBC}(1)$ under Subgroup N1.

Boundary Condition Type

NTIME, BCTYPM, BCTYPF, BCMTH

I10, 3A10

NTIME is the number of data pairs or triplets in the table of boundary condition data for the left face pointing in the $z$-direction (see Subgroup $\mathrm{P} 4$ ).

BCTYPM is the type of porous medium boundary condition applied to the left face pointing in the $z$-direction. The choices are the same as those for Subgroup N2.

BCTYPF is the type of fracture boundary condition applied to the left face pointing in the $z$-direction. The choices are the same as those for Subgroup N2.

BCMTH tells the code how to distribute the boundary conditions between the porous medium and fractures for the left face pointing in the $z$-direction. The choices are the same as those for Subgroup N2 except that references to Subgroup N4 are to Subgroup P4 instead.

Boundary Condition Range

IMIN, IMAX, JMIN, JMAX

$4 \mathrm{I} 10$

IMIN is the minimum index in the $x$-direction of the boundary condition applied to the left face pointing in the $z$-direction.

IMAX is the maximum index in the $x$-direction of the boundary condition applied to the left face pointing in the $\mathrm{z}$-direction.

JMIN is the minimum index in the y-direction of the boundary condition applied to the left face pointing in the $z$-direction.

JMAX is the maximum index in the y-direction of the boundary condition applied to the left face pointing in the $z$-direction.

IMIN and IMAX must be between 1 and NXX. If NXX is 0 , set IMIN and IMAX to 1 . JMIN and JMAX must be between 1 and NYY. If NYY is 0 , set JMIN and JMAX to 1 .

Boundary Condition Data

BCTIM, BCMAT, BCFRC

3E10. 0
Variables:

Format: 
Description:

Subgroup P5:

Variables:

Format:

Description:

Subgroup P6:

Variables:

Format:

Description:

Subgroup P7:

Variables:

Format:
BCTIM is the time at which the boundary condition is applied to the left face pointing in the $z$-direction.

BCMAT is, under most conditions, the value of porous medium boundary condition applied to the left face pointing in the $z$-direction at the time BCTIM. The description for BCMAT is the same as that described for Subgroup N4.

BCFRC is the value of the fracture boundary condition applied to the left face pointing in the $z$-direction at the time BCTIM. The description for BCFRC is the same as that described for Subgroup N4.

The conditions applied to Subgroup $\mathrm{P} 4$ are the same as those applied to Subgroup N4. Subgroup $\mathrm{P} 4$ is provided NTIME (Subgroup P2) times.

Subgroups P2, P3, and P4 are provided NBC(5) times. If NBC (5) is set to 0 , then Subgroups P2, P3, and P4 are not provided.

Boundary Condition Numbers for Y-Direction, Right Face $\operatorname{NBC}(6)$

I10

NBC(6) is the number of non-zero flux and pressure boundary condition tables on the right face of the boundary pointing in the $z$-direction. The description of $\mathrm{NBC}(6)$ is the same as $\mathrm{NBC}(1)$ under subgroup $\mathrm{N} 1$.

Boundary Condition Type

NTIME, BCTYPM, BCTYPF, BCMTH I10, 3A10

NTIME is the number of data pairs or triplets in the table of boundary condition data for the right face pointing in the $z$-direction (see Subgroup P8).

BCTYPM is the type of porous medium boundary condition applied to the right face pointing in the $z$-direction. The choices are the same as those for subgroup N2.

BCTYPF is the type of fracture boundary condition applied to the right face in the $\mathrm{z}$-direction. The choices are the same as those for Subgroup N2.

BCMTH tells the code how to distribute the boundary conditions between the porous medium and fractures for the right face pointing in the $z$-direction. The choices are the same as for Subgroup N2 except that references to Subgroup $\mathrm{N} 4$ are to subgroup P8 instead.

Boundary Condition Range IMIN, IMAX, JMIN, JMAX $4 \mathrm{I} 10$ 
Description: IMIN is the minimum index in the $x$-direction of the boundary condition applied to the right face pointing in the $\mathrm{z}$-direction.

IMAX is the maximum index in the $x$-direction of the boundary condition applied to the right face pointing in the $\mathrm{z}$-direction.

JMIN is the minimum index in the y-direction of the boundary condition applied to the right face pointing in the $z$-direction.

JMAX is the maximum index in the $y$-direction of the boundary condition applied to the right face pointing in the $z$-direction.

Conditions on IMIN, IMAX, JMIN, and JMAX are the same as those for Subgroup P3.

Subgroup P8:

Variables:

Format:

Description:
Boundary Condition Data

BCTIM, BCMAT, BCFRC

$3 \mathrm{E} 10.0$

BCTIM is the time at which the boundary condition is applied to the right face pointing in the $z$-direction.

BCMAT is, under most conditions, the value of the porous medium boundary condition applied to the right face pointing in the $\mathrm{z}$-direction at time BCTIM. The description for BCMAT is the same as that described for Subgroup $\mathrm{N} 4$.

BCFRC is the value of the fracture boundary condition applied to the right face pointing in the $z$-direction at time BCTIM. The description for BCFRC is the same as that described for Subgroup N4.

Conditions applied to Subgroup P8 are the same as those applied to Subgroup N4. Subgroup P8 is provided NTIME (Subgroup P6) times.

Rules and choices applicable to Subgroups N2, N3, and N4 are applicable tó Subgroups $\mathrm{P} 6, \mathrm{P} 7$, and $\mathrm{P} 8$, respectively.

Subgroups $\mathrm{P} 6, \mathrm{P} 7$, and $\mathrm{P} 8$ are provided $\mathrm{NBC}(6)$ times. If $\mathrm{NBC}(6)$ is set to 0 , then Subgroups $P 6, P 7$, and $P 8$ are not provided.

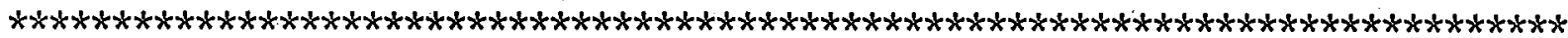

Group Q: Source Term Data Records

Subgroup Q1: 'Source Term Numbers

Variables: NSRC

Format: $\quad$ I10 
Description:

Subgroup Q2: Variables:

Format:

Description:

NSRC is the number of source term tables in the modeled region. If there are no source terms, then set NSRC to 0 . Otherwise, set NSRC to the number of source terms tables. If NSRC is set to 0 , subgroups Q2, Q3, and Q4 are not read and should not be included in the input file.

Source Term Type NTIMES, SRCMTH $\mathrm{I} 10, \mathrm{~A} 10$ NTIMES is the number of data pairs or triplets in the table of source term data (see Subgroup Q4).

SRCMTH tells the code how to distribute the source term between the porous medium and fractures.

If OPTION(2) is set to DOUBLE, the choice is either PRESCRIBED or MOBILITY. PRESCRIBED is set if the source term is described by the user and provided in the table below (see Subgroup Q4). MOBILITY is set if the source term is distributed between the porous medium and fractures based on the mobility coefficients of the porous medium and fractures.

If OPTION(2) is set to SINGLE, then DCM3D defaults SRCMTH to PRESCRIBED.

Figure 5-4 will aid in the proper selection of SRCMTH.

Subgroup Q3: Variables: Format: Description:
Source Term Range

IMIN, IMAX, JMIN, JMAX, KMIN, KMAX

$6 \mathrm{I} 10$

IMIN is the minimum index in the $x$-direction of the source term table.

IMAX is the maximum index in the $x$-direction of the source term table.

JMIN is the minimum index in the y-direction of the source term table.

JMAX is the maximum index in the $y$-direction of the source term table.

KMIN is the minimum index in the z-direction of the source term table.

KMAX is the maximum index in the $z$-direction of the source term table.

IMIN and IMAX must be between 1 and NXX. If NXX is 0 , set IMIN and IMAX to 1 . JMIN and JMAX must be between 1 and NYY. If NYY is 0 , set JMIN and JMAX to 1 . KMIN and 


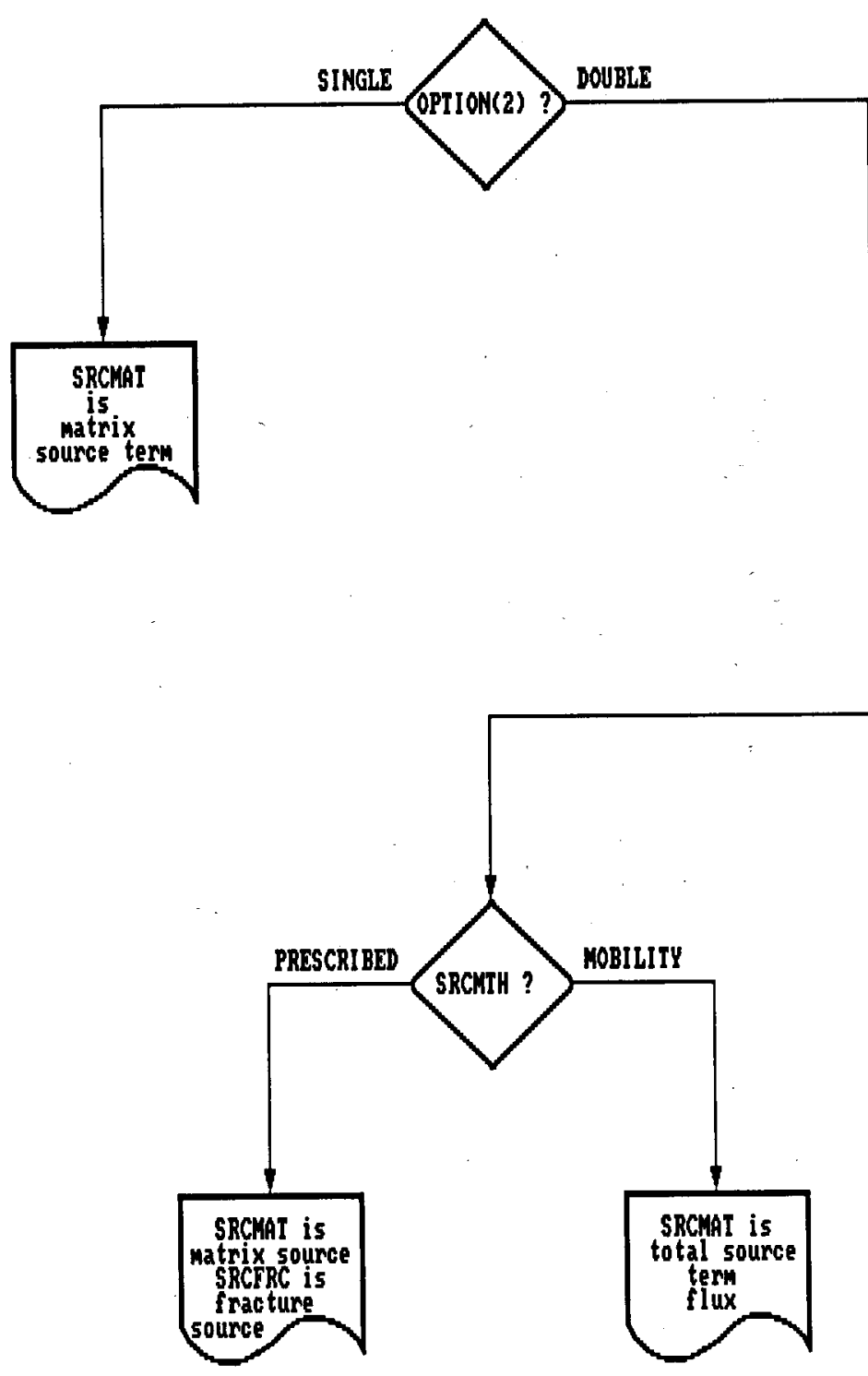

Figure 5-4: Flow Chart for Selecting Source Terms

- Page 74 - 
KMAX must be between 1 and NZZ. If NZZ is 0 , set KMIN and KMAX to 1 .

Subgroup Q4:

Variables:

Format:

Description:
Source Term Table Data

SRCTIM, SRCMAT, SRCFRC

$3 \mathrm{E} 10.0$

SRCTIM is the time at which the source term is applied (s).

SRCMAT is, under most conditions, the value of the source term for the porous medium at the time SRCTIM. However, if SRCMTH is set to MOBILITY, then SRCMAT is the total volumetric source $\left(\mathrm{m}^{3} / \mathrm{s}\right)$ into both the porous medium and fractures for the grid block. A positive value indicates flow into the grid block. A negative value indicates removal from the grid block. If Subgroup Q4 is required, a value must be supplied here. If no value is supplied, DCM3D will set SRCMAT to $0.0\left(\mathrm{~m}^{3} / \mathrm{s}\right)$.

SRCFRC is the value of the source term for the fractures at the time, SRCTIM. If SRCMTH is set to PRESCRIBED, a value must be supplied here. If no value is supplied, the code will set SRCFRC to $0.0\left(\mathrm{~m}^{3} / \mathrm{s}\right)$. However, if SRCMTH is set to MOBILITY, then no value needs to be specified. The code internally calculates the fracture source term based on the SRCMAT values and the mobility ratios.

Figure 5-4 will aid in the selection of SRCMAT and SRCFRC values. Figure 5-5 provides a flow chart for reading the source term input data.

Subgroup Q4 is provided NTIMES times.

Subgroup Q4 specifies a table of source terms for which the following rules apply.

1. If simulation time, $t$, is less than $\operatorname{SRCTIM(1)}$, then the source term values are set to 0.0 .

2. If simulation time is between SRCTIM(i) and $\operatorname{SRCTIM}(i+1)$, then the source term values are determined from a linear interpolation.

3. If simulation time is larger than SRCTIM(NTIMES), then the source term values are set to the last value in the table.

Subgroups Q2, Q3, and Q4 are provided NSRC times. If NSRC is set to 0 , then Subgroups Q2, Q3, and Q4 are not provided. 


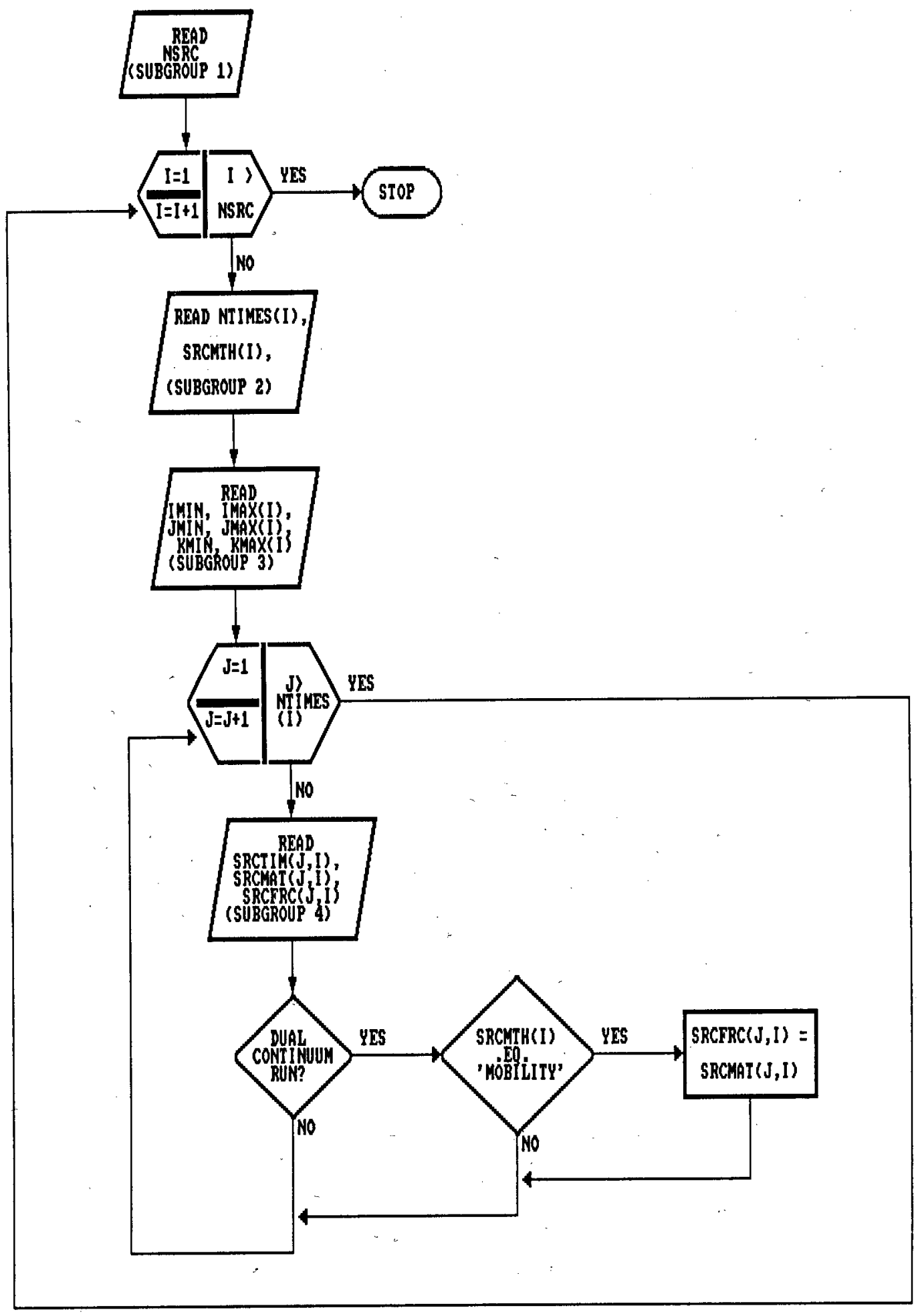

Figure 5-5: Flow Chart for Reading Source Terms 


\subsection{Data Input for a Restart Run}

DCM3D possesses the capability to run problems from a restart file. Running from a restart may be done for several reasons. In one case, a problem may exceed its CPU time. If this happens and if the analyst wants to continue the problem, he can use the data in the restart file and continue where the problem left off. In another case, the analyst may want to run a problem to steady-state for a certain condition (e.q. a fixed recharge rate). He may then want to run several transient solutions with different values of recharge rates from that steady-state solution.

In order to run a restart problem, two types of input files must exist. These are a modified input file and a restart input file. The modified input file is similar to the input file for an initial run. There are some exceptions, though. Instructions for creating this modified input file follow. The restart input file is generated by a previous run. It is created by specifying a save file name on the FILES record (see Group D in Section 5.2) and SAVE on the WRITE TIME DATA records (see Subgroup K2 in Section 5.2). The restart file for a run is the same as the save file created by a previous run.

The modified input file consists of the following data input groups from Section 5.2.

Group A: Restart (this group is described below and replaces the title group)

Group D: Files

Group E: Options (this group is modified as described below)

Group I: Time Step Data

Group K: Write Times

Group N: X-Direction Boundary Conditions Data

Group 0: Y-Direction Boundary Conditions Data

Group P: Z-Direction Boundary Conditions Data

Group Q: Source Term Data

The restart input file contains the following data, which are written to it by a previous run.

$\begin{array}{ll}\text { Group A: } & \text { Title } \\ \text { Group B: } & \text { Subtitle } \\ \text { Group C: } & \text { Analyst } \\ \text { Group D: } & \text { Options (contains only SINGLE or DOUBLE) } \\ \text { Group F: } & \text { Water Data } \\ \text { Group G: } & \text { Gravity Data } \\ \text { Group H: } & \text { Convergence Criteria } \\ \text { Group J: } & \text { Grid Block Data } \\ \text { Group L: } & \text { Material Data } \\ \text { Group M: } & \text { Initial Pressure Conditions }\end{array}$

\subsubsection{Input File Modifications to Group A}

Group A: Restart Record

Subgroup A1: $\quad$ Restart

Variables: $\quad$ AREST, FILRES

Format: $\quad 2 \mathrm{AlO}$ 
Description: AREST is a variable used to test for a restart run. If AREST is set to RESTART, DCM3D knows that this is a restart run and will try to access both a modified input file and a restart file. If AREST is set to anything else, DCM3D assumes that this run is a regular run.

FILRES is the name of the restart file. An existing restart file name must be provided here. If FILRES is left blank, DCM3D will not know where to look for a restart file. DCM $3 D$ will then print a message and stop running.

(Note: The problem title, which is read on this record during an initial run, is stored in the restart file.)

\subsubsection{Input File Modification to Group D}

Group D: Options Record

Subgroup D1:

Variables:

Format:

Description:
Options

OPTION(1)

A10

OPTION(1) tells DCM3D to actually run the problem. Set OPTION(1) to RUN if the restart problem is to run the problem after reading both the modified input file and the restart file. If OPTION(1) is set to anything else, including a blank, only a data input check will be performed.

(Note: OPTION(2), which is read on this record during an initial run, is stored in the restart file.)

\subsubsection{Other Modifications to Input Files}

The start time for a restart run is specified as TSTART on the TIME STEP DATA records (Group I). The start time can be any positive value including 0.0 . If the start time is greater than 0.0 , the analyst should make certain that the write times (TWRITE in Group $\mathrm{K}$ ), are all greater than TSTART. In addition, the boundary condition times (BCTIM in Groups $N$, 0 , and $P$ ) and the source term times (SRCTIM in Group Q) are all absolute values with respect to TSTART. These times are not relative to TSTART.

\section{$5.4 \quad$ Dimensioning Arrays}

DCM3D uses FORTRAN "PARAMETER" statements to dimension arrays. Thus, it is very easy to change array sizes in DCM3D. In order to change array sizes, "PARAMETER" statements in the main routine and subroutine DYDTEQ must be changed. Some of the parameters exist in both the main routine and subroutine DYDTEQ. It is very important that the same parameter values in both routines agree. If they do not, DCM3D will not run correctly. If an array is too small for the amount of data supplied, DCM3D will print a 
message informing the analyst to increase the appropriate parameter, and then stop running.

\section{$5.4 .1 \mathrm{MAXBC}$}

Parameter MAXBC specifies the maximum number of boundary condition tables on any side of the grid. This parameter appears in both the main routine and subroutine DYDTEQ. Therefore, the values for MAXBC must agree in both routines.

\subsubsection{MAXDX}

Parameter MAXDX is used to set the maximum number of grid blocks in the $x$ direction. This parameter appears in both the main routine and subroutine DYDTEQ. Therefore, the values for MAXDX must agree in both routines.

\subsubsection{MAXDY}

Parameter MAXDY is used to set the maximum number of grid blocks in the $y$ direction. This parameter appears in both the main routine and subroutine DYDTEQ. Therefore, the values for MAXDY must agree in both routines.

\section{$5.4 .4 \mathrm{MAXDZ}$}

Parameter MAXDZ is used to set the maximum number of grid blocks in the $z$ direction. This parameter appears in both the main routine and subroutine DYDTEQ. Therefore, the values for MAXDZ must agree in both routines.

\subsubsection{MAXLRW}

Parameter MAXLRW is used to dimension the work array used by LSODES. This parameter appears only in the main routine. MAXLRW is estimated by an equation from MAXDX, MAXDY, MAXDZ, and the real word and integer word lengths of the particular computer system being used. This equation may not always provide a reliable estimate of the actual value of MAXLRW. If MAXLRW is too small, LSODES will print a diagnostic message and cause DCM3D to stop running. If this occurs, replace the equation in the PARAMETER statement that calculates MAXIRW with number provided by the LSODES message. This parameter appears only in the main routine.

\subsubsection{MAXMAT}

Parameter MAXMAT is used to specify the maximum number of materials. This parameter appears in both the main routine and subroutine DYDTEQ.

Therefore, the values for MAXMAT must agree in both routines. 


\subsubsection{MAXSRC}

Parameter MAXSRC is used to specify the maximum number of source terms. This parameter appears in both the main routine and subroutine DYDTEQ. Therefore, the values for MAXSRC must agree in both routines.

\subsubsection{MAXTIM}

Parameter MAXTIM is used to specify the maximum number of times in a boundary condition or source term table. This parameter appears in both the main routine and subroutine DYDTEQ. Therefore, the values for MAXTIM must agree in both routines.

\subsubsection{MAXWRT}

Parameter MAXWRT is used to specify the maximum number of write times for a run. This parameter appears only in the main routine.

\subsection{Running with Head}

Although DCM3D is designed to run in terms of pressure, it can easily be run in terms of pressure head. Only the a few variables in the input file are affected when running DCM3D in terms of pressure head. Modifications to the variables in the input file include:

1. In Group F (Water Data):

Set DENS to 1.0 (dimensionless).

Set VISC to 1.0 (dimensionless).

Change COMP from inverse pressure units (e.g. $\mathrm{Pa}^{-1}$ ) to inverse head units (e.g. $\left.\mathrm{m}^{-1}\right)$. This is done by multiplying COMP in inverse pressure units by water density and the gravitational coefficient.

2. In Group G (Gravity):

Set GRAV to 1.0 (dimensionless)

3. In Group H (Convergence Criteria):

Change ATOL from a pressure unit (e.g. Pa) to a head unit (m). This is done by dividing ATOL in pressure units by water density and the gravitational coefficient.

4. In Group L (Material Data):

Change COMPR (Subgroups L3 and L5) from inverse pressure units to inverse head units. This is done by multiplying 
COMPR in inverse pressure units by water density and the gravitational coefficient.

Change PERMXX, PERMYY, and PERMZZ (Subgroups L3 and L5)

from permeability units $\left(\mathrm{e} . \mathrm{g} . \mathrm{m}^{2}\right.$ ) to hydraulic conductivity units (e.g. m-s $\left.{ }^{-1}\right)$. This is done by multiplying PERMXX, PERMYY, and PERMZZ in permeability units by water density and the gravitational coefficient and dividing by water viscosity.

Change ALPHA (Subgroups L4 and L6) from inverse pressure units to inverse head units. This is done by multiplying ALPHA in inverse pressure units by water density and the gravitational coefficient.

Change AVFL (Subgroup L6) from dimensionless units to inverse length-time units (e.g. $\mathrm{m}^{-1}-\mathrm{s}^{-1}$ ). This is done by multiplying AVFL by water density and the gravitational coefficient and dividing by water viscosity.

5. In Group M (Pressure Initial Conditions):

Change PRESS from pressure units to pressure head units. This is done by dividing PRESS in pressure units by water density and the gravitational coefficient.

6. In Group N (X-Direction Boundary Conditions):

Change any references to pressure boundary conditions from pressure units to pressure head units. This is done by dividing pressure by water density and the gravitational coefficient.

7. In Group O (Y-Direction Boundary Conditions):

Change any references to pressure boundary conditions from pressure units to pressure head units. This is done by dividing pressure by water density and the gravitational coefficient.

8. In Group $\mathrm{P}$ (Z-Direction Boundary Conditions):

Change any references to pressure boundary conditions from pressure units to pressure head units. This is done by dividing pressure by water density and the gravitational coefficient.

\subsection{Plot File Structure}

The plot file is designed to be used for post-processing output data from DCM3D. Grid spacing, material data, grid spacing, pressure, moisture content or saturation, and Darcy velocities are all written to this file. 
The file is written with FORTRAN formatted output into a text file so that it can be read by an analyst or another computer code.

The FORTRAN computer code, READPL, presented in Table 5-1 will read the plot file. This computer code can be modified by the analyst to manipulate the data as the analyst sees fit. For instance, the computer program can be modified to write the output data so that it can be processed by a spreadsheet. The spreadsheet program can be used to make graphics of the output. In addition, READPL could be modified to make graphics of the output directly. In another case, READPL can be modified to plot data to a plot device attached to the analyst's computer.

\subsection{Compiling, Linking, and Running DCM3D}

Since DCM3D is written in standard FORTRAN, it can be used on almost any computer. Therefore, only general instructions for compiling, linking and running DCM3D are presented here.

The DCM3D computer code is supplied as two files. DCM3D.FOR is the FORTRAN code that reads the input data, calculates the differential equations for LSODES, and writes the output files. LSODES.FOR solves the differential equations created by DCM3D.FOR.

In order to run DCM3D, do the following:

1. Compile DCM3D.FOR and LSODES.FOR. Compile each file separately. DCM3D will need to be compiled each time the array dimensions are changed. LSODES.FOR needs to be compiled only once because its array dimensions are passed as arguments from DCM3D. FOR .

2. After DCM3D.FOR and LSODES.FOR are compiled, link the object files created from DCM3D.FOR and. LSODES.FOR together. Use the appropriate link or load routine for the computer being used. Specify the DCM3D.FOR object file first on the link control statement.

3. Create an input file and name it INPUT. Because DCM3D is written in standard FORTRAN, all file names used by DCM3D and other alphabetic characters should be capitalized.

4. Run the DCM3D executable program with the appropriate run command for the computer system being used.

DCM3D uses three system dependent routines that will most likely not work on other computers. In the supplied version of DCM3D, these routines are CLOCK, which returns CPU time since the start of the run; DATE, which returns the current date at the start of the run; and TIME, which returns the clock time at the start of the run. These system routines may have to be changed if DCM3D is run on different computers. 
program readp1

c

c

c

c

c

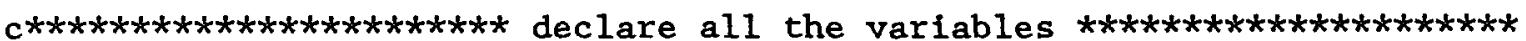
c....characters

character*5 matnam

character $* 10$ amoist, ave 1

character $* 10$ opt2

character $* 80$ subtit

character $* 80$ title

c

c... integers

integer i, iplot, iout

integer $j$

integer $k$

integer matnum, maxmat, $\operatorname{maxdx}, \operatorname{maxdy}, \operatorname{maxdz}$

integer nummat

integer $n x, n x x, n y, n y y, n z, n z z$

c

c...reals

real cntntf

real cntntm

real $d x, d y, d z$

real frcprp

real matprp

real pressf, pressm

real qxf, qxm, qyf, qym, qzf, qzm

real time

c

c

c

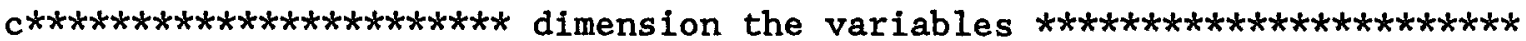

c...set up maximum dimensions

parameter $(\max d x=150, \max d y=20, \max d z=1, \operatorname{maxmat}=20$ )

c

dimension cntntf(maxdx, maxdy, maxdz)

dimension cntntm(maxdx, maxdy, maxdz)

dimension $\mathrm{dx}(\max \mathrm{dx})$

dimension dy (maxdy)

dimension $\mathrm{dz}(\operatorname{maxdz})$

dimension frcprp (11, maxmat)

dimension matnam (maxmat)

dimension matnum $(\max d x, \operatorname{maxdy}, \operatorname{maxdz})$

dimension matprp (11, maxmat)

dimension pressm(maxdx, $\operatorname{maxdy}, \operatorname{maxdz})$

dimension pressf (maxdx, maxdy, maxdz)

dimension $q x m(\operatorname{maxdx}+1, \operatorname{maxdy}, \operatorname{maxdz})$

dimension $q x f(\operatorname{maxd} x+1, \operatorname{maxdy}, \operatorname{maxd} z)$

dimension $q y m(\max d x, \max d y+1, \operatorname{maxd} z)$ 
Table 5-1: FORTRAN Program READPL (continued)

dimension $q y f(\max d x, \max d y+1, \operatorname{maxd} z)$

dimension $q z m(\max d x, \operatorname{maxdy}, \operatorname{maxdz}+1)$

dimension $q z f(\operatorname{maxdx}, \operatorname{maxdy}, \operatorname{maxd} z+1)$

c

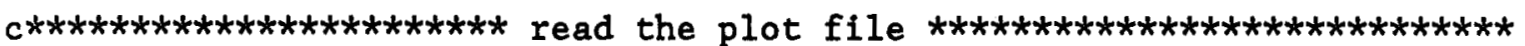

c...open the read file

parameter (iplot $=5$, lout $=6$ )

open (unit =iplot, file $=$ 'plotpl', status $={ }^{\prime} O L D^{\prime}$ )

c...open an output file

c open(unit=iout, file='output', status='UNKNOWN')

c

c...read title record

read(iplot,'(a)') title

c

c...read subtitle record

read(iplot,'(a)') subtit

c

c...read options record

read(iplot,' $\left.(2 a)^{\prime}\right)$ opt2

c

c...read grid block numbers record

read(iplot, *) nxx, nyy, nzz

if ( $\operatorname{maxdx} .1 t . n \times x)$ then

write (iout,' $(2 a, 15, a) ')$ ' $* * *$ increase parameter ' 'maxdx' ',

$\&$

'to at least', $\max 0(1, \mathrm{nxx}),{ }^{\prime} * * *$ '

endif

if (maxdy .1t. nyy) then

write(iout,' $(2 a, 15, a) '$ ) ' $\star \star \star *$ increase parameter ' 'maxdy' ' ',

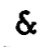

'to at least', max $0(1$, nyy $), ' * * * '$

endif

if $(\operatorname{maxdz} .1 \mathrm{t}, \mathrm{nzz})$ then

write (iout,' $\left.(2 a, 15, a)^{\prime}\right)$ ' $* * \star$ increase parameter ' 'maxdz' ',

$\&$

'to at least ' $, \max 0(1, \mathrm{nzz}),,^{\prime} * *{ }^{\prime}$

endif

c

c...read grid spacing

if ( $\mathrm{nxx} . \mathrm{gt} .0$ ) then

$\operatorname{read}(i p l o t, *)(d x(i), i=1, n \times x)$

$\mathrm{nx}=\mathrm{nxx}$

else

$\mathrm{dx}(1)=1.0$

endif

$\mathrm{nx}=1$

c
if (nyy .gt. 0) then
$\operatorname{read}(i p l o t, *)(\operatorname{dy}(i), i=1$, nyy)
ny=nyy
else
$\operatorname{dy}(1)=1.0$ 
Table 5-1: FORTRAN Program READPL (continued)

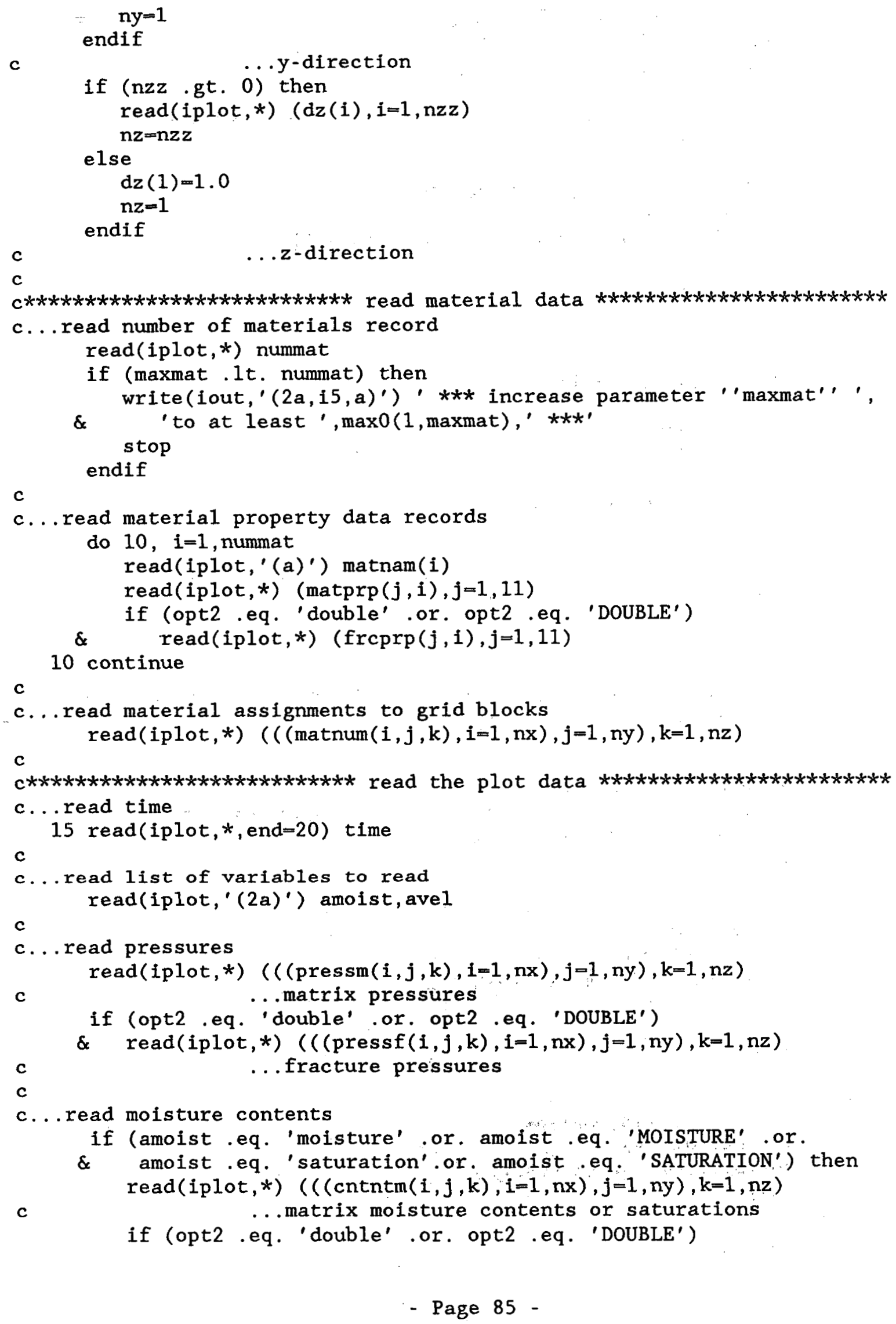


Table 5.1: FORTRAN Program READPL (concluded)

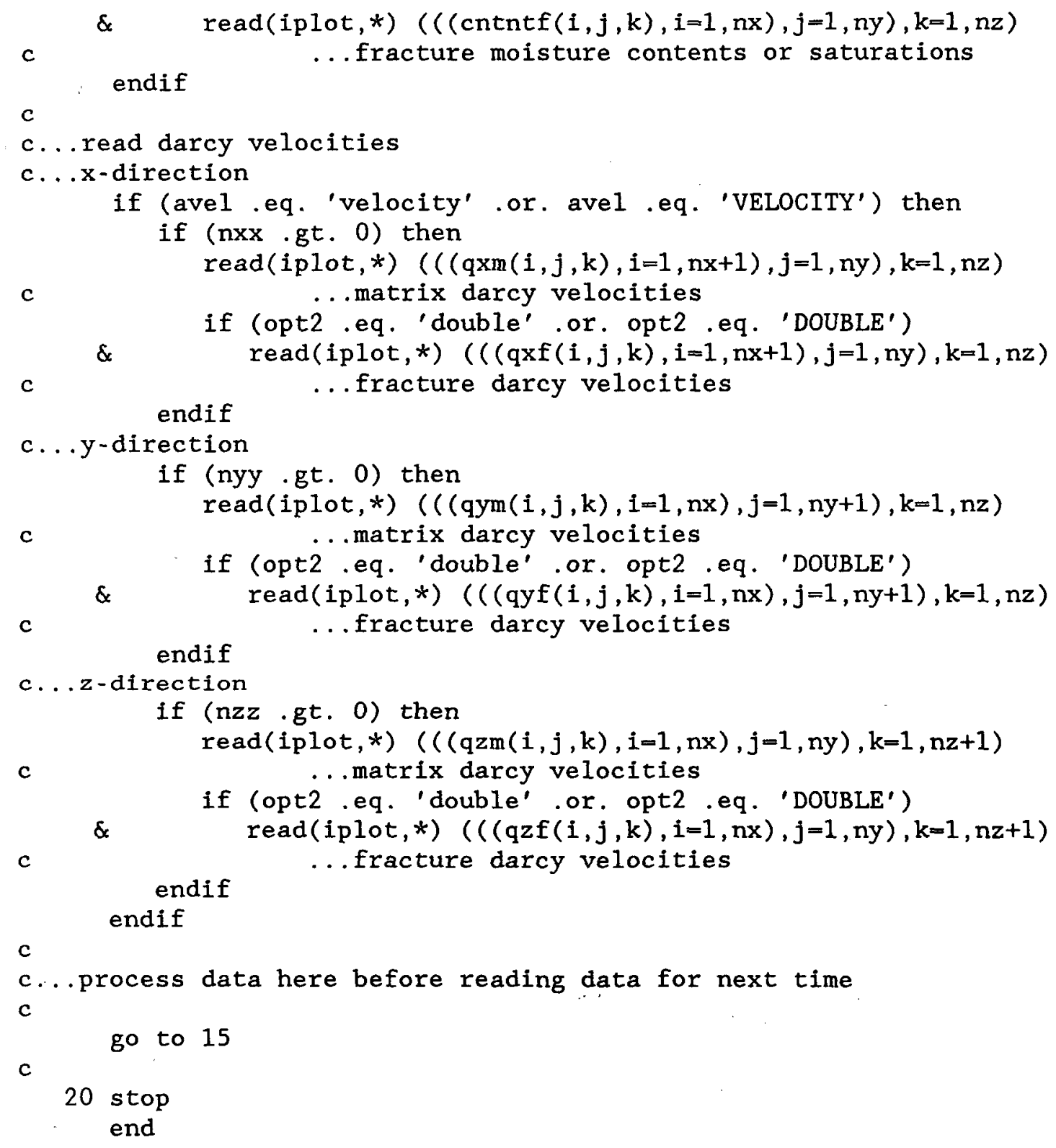


This section presents several illustrative problems for demonstrating the operation of DCM3D. Several selected problems compare the DCM3D results with analytical solutions. As a result, only relatively simple problems are presented. However, the problems do consist of both saturated and unsaturated flow problems, and single and double continuum flow problems. Problems involving flow in a unsaturated, fractured, porous medium are too difficult to solve with analytical solutions, so comparisons with the flow code for these types of problems are not presented.

\subsection{One-Dimensional Horizontal Infiltration}

The one-dimensional', horizontal infiltration problem was originally solved by Philip (1955) and is described in Ross et al. (1982). In this problem, a semi-infinite horizontal tube filled with a homogeneous soil is partially saturated with water. The tube of soil is held saturated at one end and, as time progresses, the moisture at the saturated end is pulled into the porous medium by capillary forces. The moisture in the tube begins to increase with respect to distance and time. The purpose of this problem is to test the code's ability to simulate transient unsaturated flow in a porous medium. A schematic of the problem is presented in Figure 6-1.

The following data are applicable to this problem:

initial moisture content saturated moisture content hydraulic conductivity porosity

$$
\begin{aligned}
& 0.20 \\
& 0.45 \\
& 1.1574 \times 10^{-7} \mathrm{~m} / \mathrm{s} \text { (1 cm/day) } \\
& 0.45
\end{aligned}
$$

The relative hydraulic conductivity-moisture content and the capillary pressure-moisture content characteristic curves are described by linear relationships as shown in Figure 6-2. This problem was run in terms of pressure and the data specified by Ross et al. (1982) were changed to reflect pressure.

Ross et al. (1982) specify the analytical solution at times of $864 \mathrm{~s}$ ( 0.01 days), $5184 \mathrm{~s}$ ( 0.06 days), and $9504 \mathrm{~s}$ ( 0.11 days). It is necessary to compare code predictions at these same simulation times. These results of the exact solution indicate that the semi-infinite soil tube can be represented by a finite one with a minimum length of $0.2 \mathrm{~m}$.

DCM3D successfully ran the one-dimensional horizontal infiltration problem to completion. The code required less than $0.5 \mathrm{~s}$ of. CPU time to complete the problem. The grid consisted of 40 blocks, each $0.005 \mathrm{~m}$ long. The left edge boundary pressure was set to $-0.01 \mathrm{~Pa}$. This keeps the degree of saturation slightly less than 1.0 during the simulation. Zero flux boundary conditions are specified along the right edge of the right-most block. The time at which output was desired was set to $864 \mathrm{~s}, 5184 \mathrm{~s}$, and $9504 \mathrm{~s}$ to correspond with the times presented in Ross et al. (1982). Subroutine LSODES was allowed to set its own time steps sizes internally in order to reach the desired write times. A copy of the input file is presented in Table 6-1. Because the characteristic curves for this sample 


\section{INITIAL CONDITION}

$\mathrm{S}=0.444 \ldots$

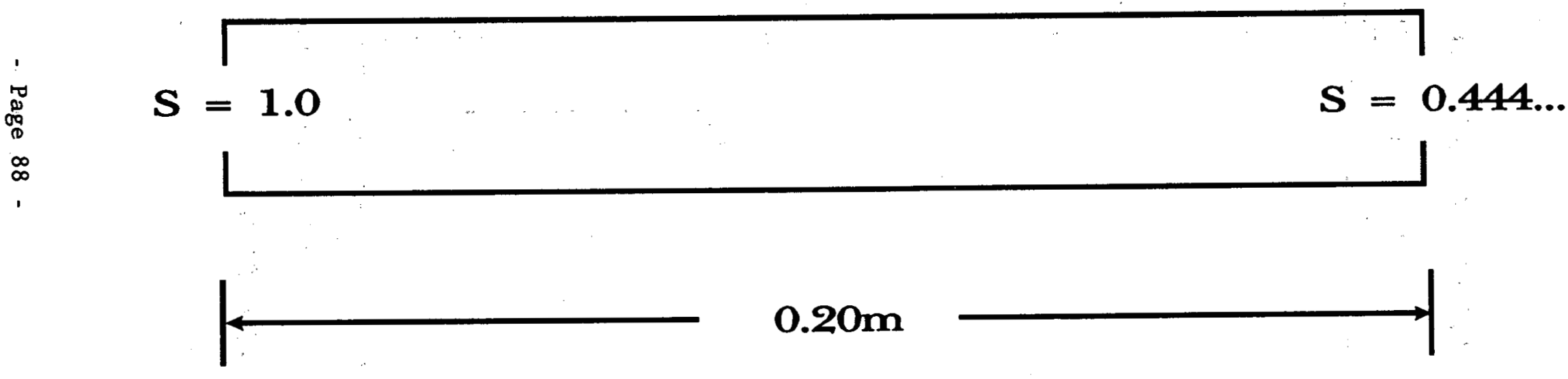

Figure 6-1: Schematic of the One-Dimensional Horizontal Infiltration Problem 


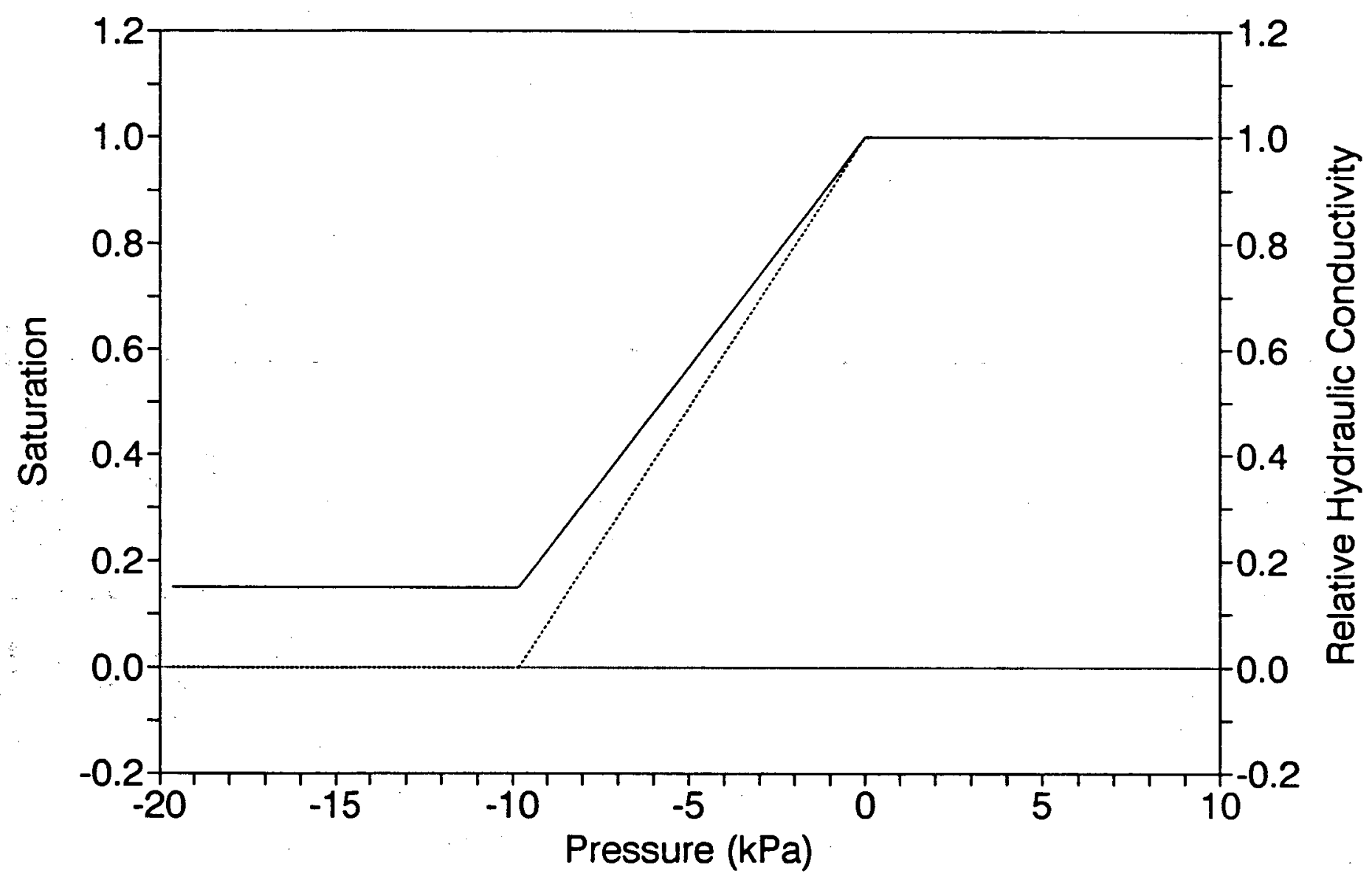

Sat.

Rel. Hydr. Cond.

Figure 6-2: Characteristic Curves for the One-Dimensional Horizontal Infiltration Problem 
Table 6-1: Input Data for the Horizontal Infiltration Problem

PHILLIP (1957) TEST RUN 12 NOV 1990

$X$-DIRECTION RUN, MATRIX ONLY

C. DAVID UPDEGRAFF

\begin{tabular}{|c|c|c|c|c|c|c|c|}
\hline RUN & SINGLE & & & & & & \\
\hline 1000 & 0.001 & 0.0 & & & & & \\
\hline 9.80665 & 0.0 & 0.0 & 0.0 & & & & \\
\hline $1.0 \mathrm{E}-4$ & 0.01 & $1.0 E-10$ & & & & & \\
\hline 5000 & 5000 & 0.0 & 0.0 & 0.0 & 0.0 & 10.0 & \\
\hline 40 & 0 & 0 & & & & & \\
\hline 0.005 & 0.005 & 0.005 & 0.005 & 0.005 & 0.005 & 0.005 & 0.005 \\
\hline 0.005 & 0.005 & 0.005 & 0.005 & 0.005 & 0.005 & 0.005 & 0.005 \\
\hline 0.005 & 0.005 & 0.005 & 0.005 & 0.005 & 0.005 & 0.005 & 0.005 \\
\hline 0.005 & 0.005 & 0.005 & 0.005 & 0.005 & 0.005 & 0.005 & 0.005 \\
\hline 0.005 & 0.005 & 0.005 & 0.005 & 0.005 & 0.005 & 0.005 & 0.005 \\
\hline & & & & & & & \\
\hline 864.0 & & PRINT & & & SATURATION & & \\
\hline 5184.0 & & PRINT & & & SATURATION & & \\
\hline $\begin{array}{r}9504.0 \\
1\end{array}$ & & PRINT & & & SATURATION & & \\
\hline SOIL & 40 & & & & & & \\
\hline 0.0 & 0.45 & $1.180 E-14$ & $1.180 \mathrm{E}-14$ & $1.180 E-14$ & 0.0 & & \\
\hline 1.0 & .33333333 & $\quad 2.0$ & & 0.5 & & & \\
\hline-8172.208 & -8172.208 & -8172.208 & -8172.208 & -8172.208 & -8172.208 & -8172.208 & -8172.208 \\
\hline-8172.208 & -8172.208 & -8172.208 & -8172.208 & -8172.208 & -8172.208 & -8172.208 & -8172.208 \\
\hline-8172.208 & -8172.208 & -8172.208 & -8172.208 & -8172.208 & -8172.208 & -8172.208 & -8172.208 \\
\hline-8172.208 & -8172.208 & -8172.208 & -8172.208 & -8172.208 & -8172.208 & -8172.208 & -8172.208 \\
\hline-8172.208 & -8172.208 & -8172.208 & -8172.208 & -8172.208 & -8172.208 & -8172.208 & -8172.208 \\
\hline 1 & & & & & & & \\
\hline 1 & PRESSURE & & & & & & \\
\hline 1 & 1 & 1 & 1 & & & & \\
\hline 0.0 & -0.01 & & & & & & \\
\hline 0 & & & & & & & \\
\hline
\end{tabular}


problem are not of the van Genuchten type, the DCM3D function subprograms which handle the characteristic curves were rewritten to handle the linear characteristic curves. The alternative function subprograms are presented in Table 6-2. The code required 90 time steps to complete the solution.

Results of the run for times of $864 \mathrm{~s}, 5184 \mathrm{~s}$, and $9504 \mathrm{~s}$ are presented in Figure 6-3. The simulation results compare extremely well with the analytical solution results for all times and distances.

\subsection{One-Dimensional Horizontal Saturated Matrix/Fracture Flow}

The one-dimensional, horizontal, saturated, matrix/fracture flow problem is designed to test the transfer term between the porous medium and fractures. In this problem, water in a highly fractured porous medium is initially at hydrostatic equilibrium. Throughout the simulation the pressure head at the left end is held fixed at a constant pressure head in both the porous medium and the fractures. At an initial time, a flux is injected into the fractures at the right end, while flux for the porous medium at the right boundary is held at 0.0 . As time proceeds the pressure in the fractures begins to increase rapidly. Because of the transfer term between the porous medium and the fractures, the pressure in the porous medium begins to increase, but at a slower rate than the pressure in the fractures. A schematic of the problem is presented in Figure 6-4. There is no published reference for this problem. The development of the analytical solution is presented in Appendix A.

This problem was run as a dimensionless one. The dimensionless rate of injection at the right boundary was set to 1.0 . The ratio of the porous medium intrinsic permeability to the fracture intrinsic permeability was 0.001 , the ratio of the porous medium storage coefficient to the fracture storage coefficient was 1000.0 , and the porous medium to fracture transfer term was 0.01 . This problem was run with parameters that produce results in terms of head. The input file is presented in Table 6-3.

The results of this simulation are presented in Figure 6-5 for the fracture solution and Figure 6-6 for the porous medium solution. The numerical solution for the fractures agrees extremely well with the analytical solution for all distances when the dimensionless time is greater than or equal to 0.01 . At smaller dimensionless times the agreement becomes poor as distance from the right boundary increases. However, this may be due more to roundoff error or convergence criteria than to a poor solution because the heads are so small in this region. The numerical solution for the porous medium agrees extremely well with the analytical solution head for all dimensionless times greater than or equal to 10.0. Porous medium heads are too small that they are meaningless for times less than 0.1

\subsection{Well Flow in a Fractured Porous Media}

The fractured well flow problem is described in Ross et al. (1982) and was originally solved by Streltsova-Adams (1978). Results of drawdowns for several sets of parameters are presented in both references. The purpose of this problem is to test the transfer term between the porous medium and 
Table 6-2: Characteristic Curve Function Subprograms for the Horizontal Infiltration Problem

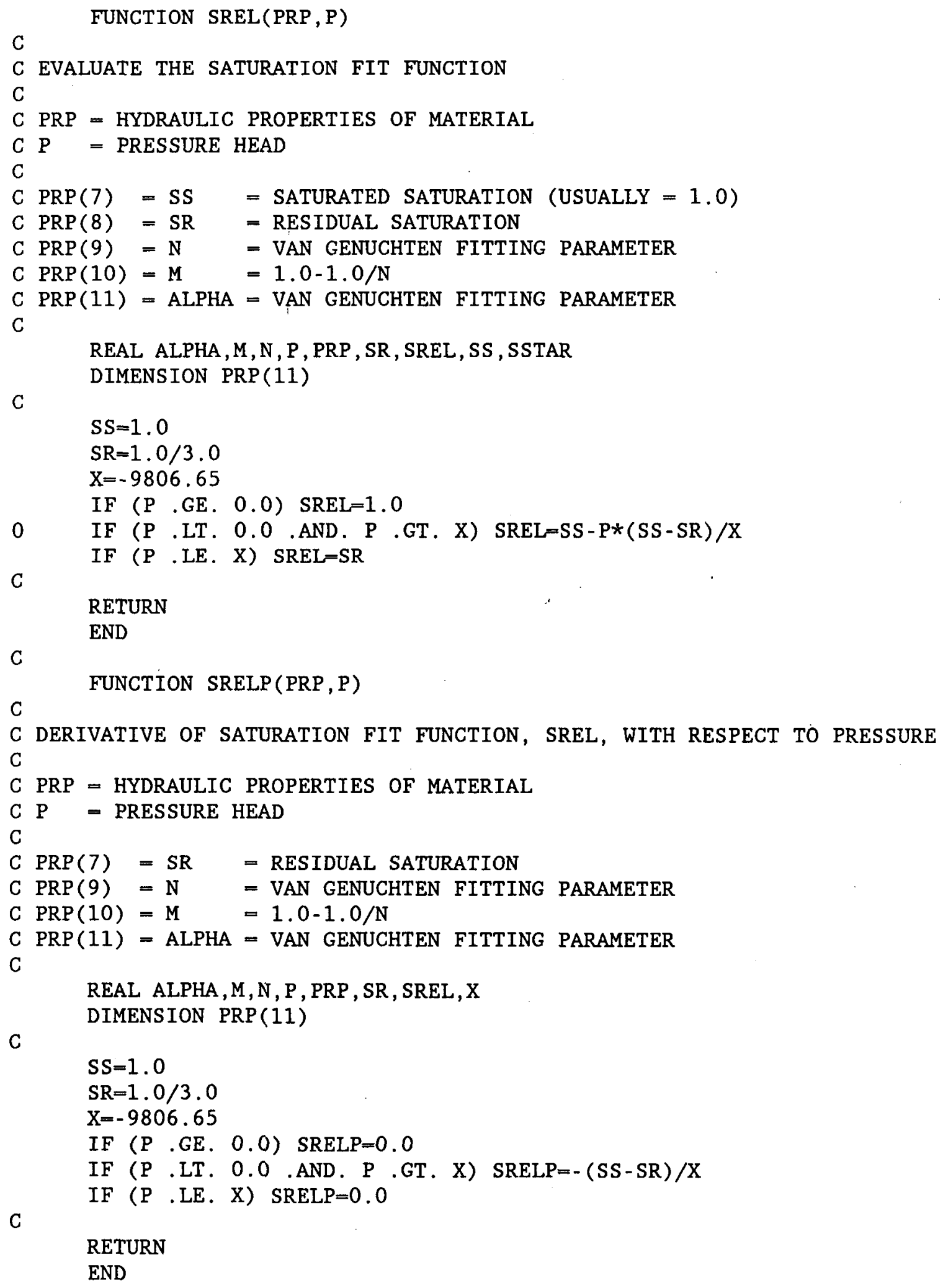


Table 6-2: Characteristic Curve Function Subprograms for the Horizontal Infiltration Problem (Continued)

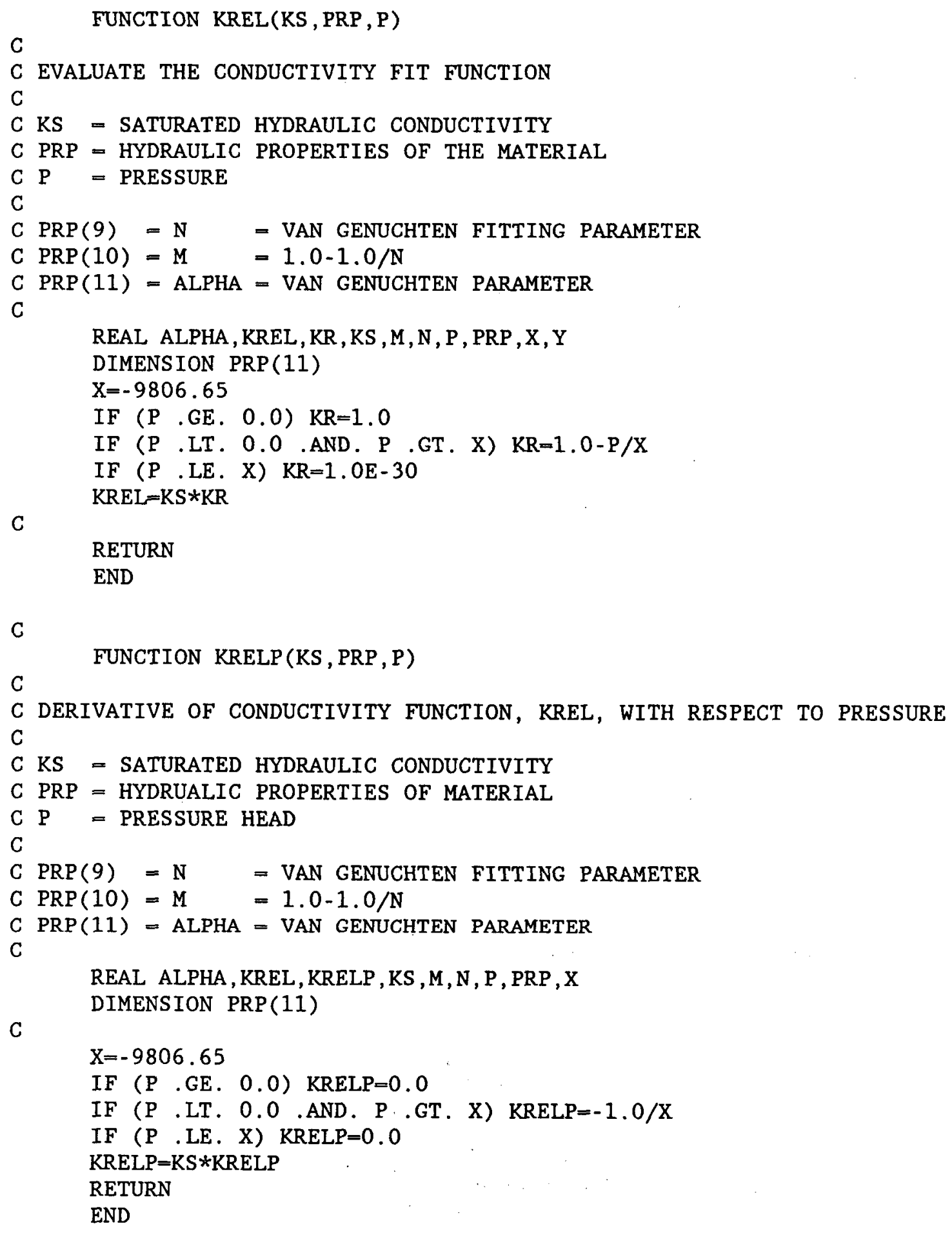




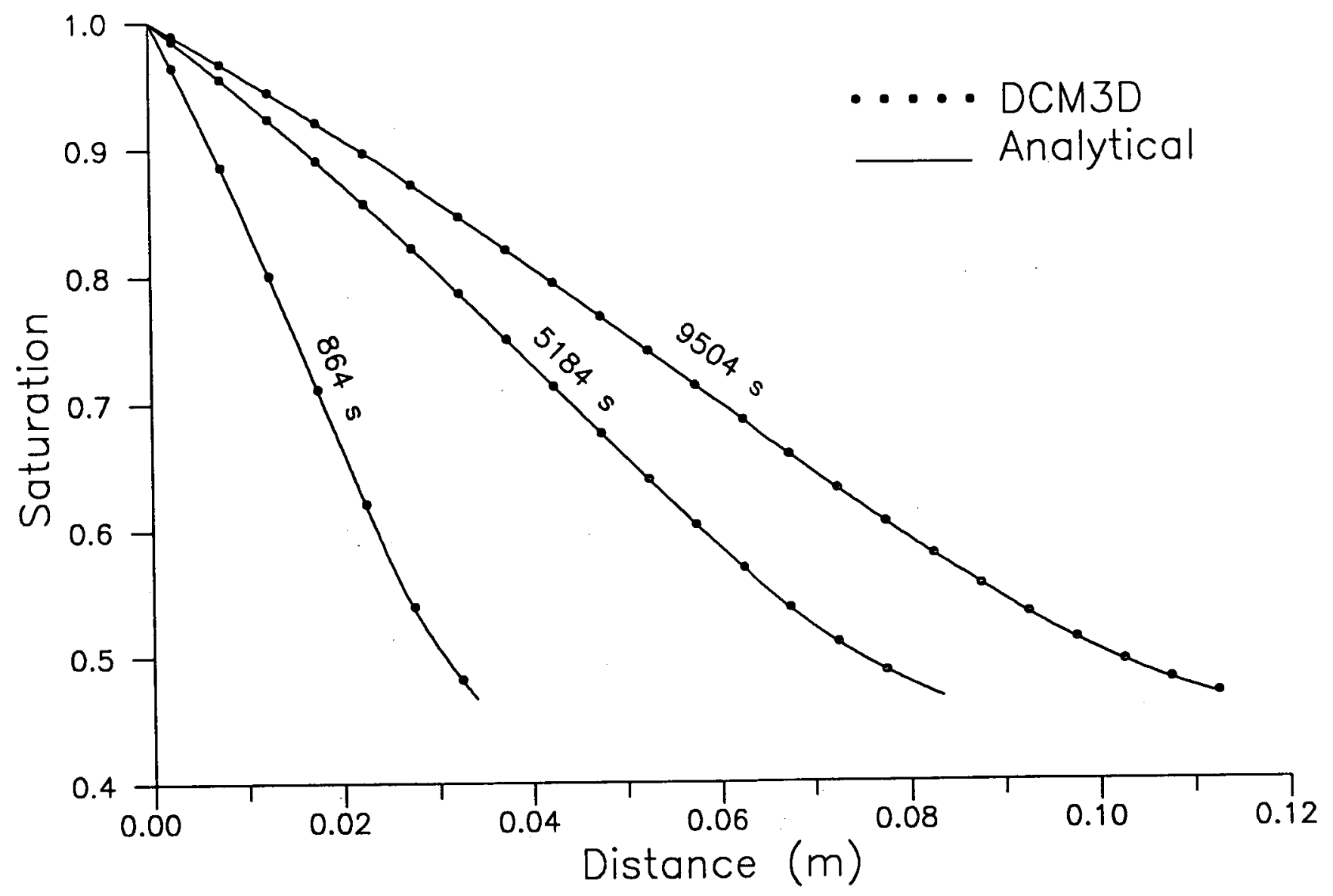

Figure 6-3: DCM3D Results for the One-Dimensional Horizontal Infiltration Problem 
1-D Saturated Fractured Porous Media

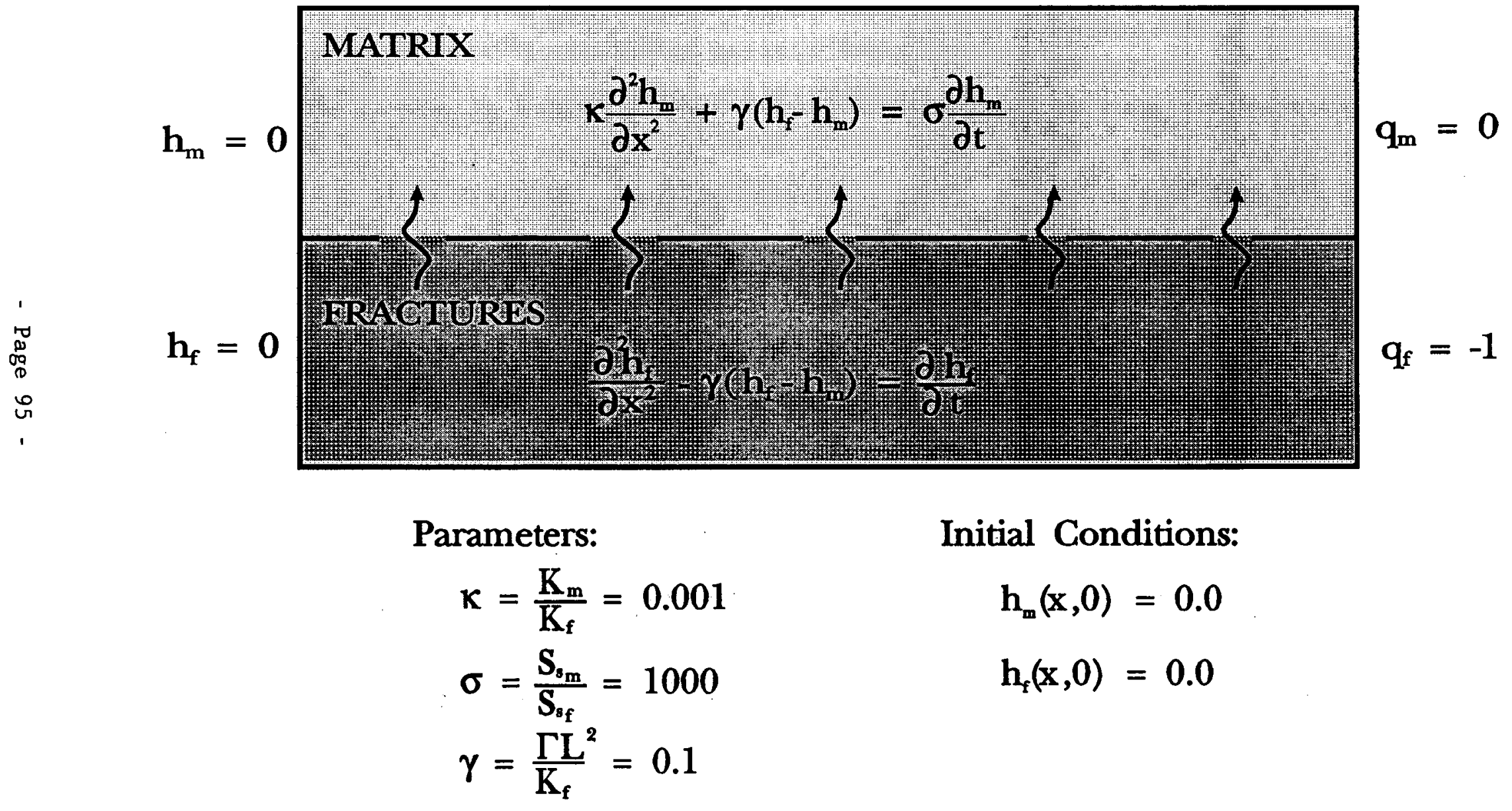

Figure 6-4: Schematic of the One-Dimensional Horizontal Saturated Matrix/Fracture Flow Problem 
1-DIMENSIONAL, FRACTURED, POROUS MEDIA TEST PROBLEM

KAPPA $=0.001$, SIGMA $=1000.0$, GAMMA $=0.1$

C. DAVID UPDEGRAFF

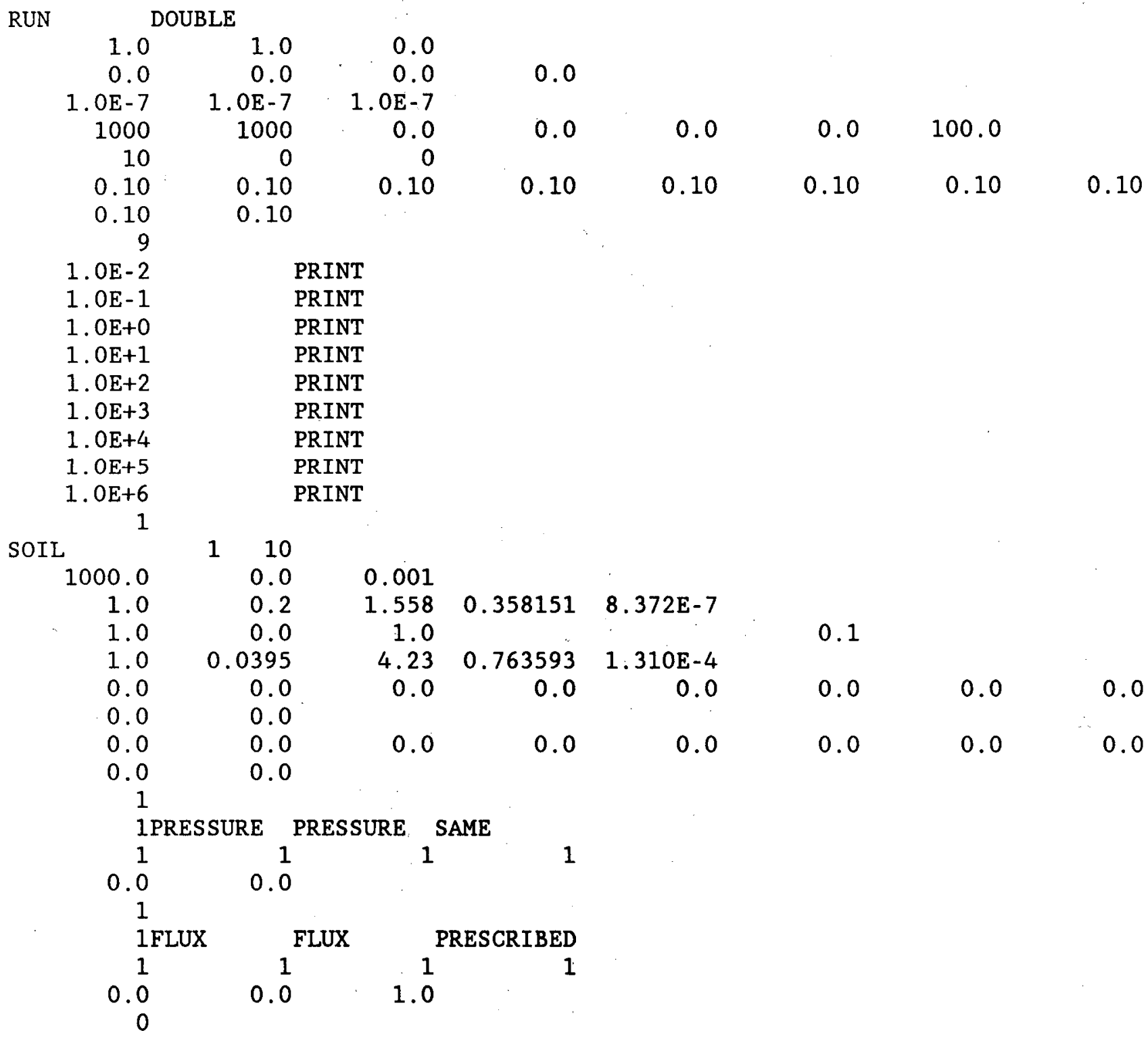




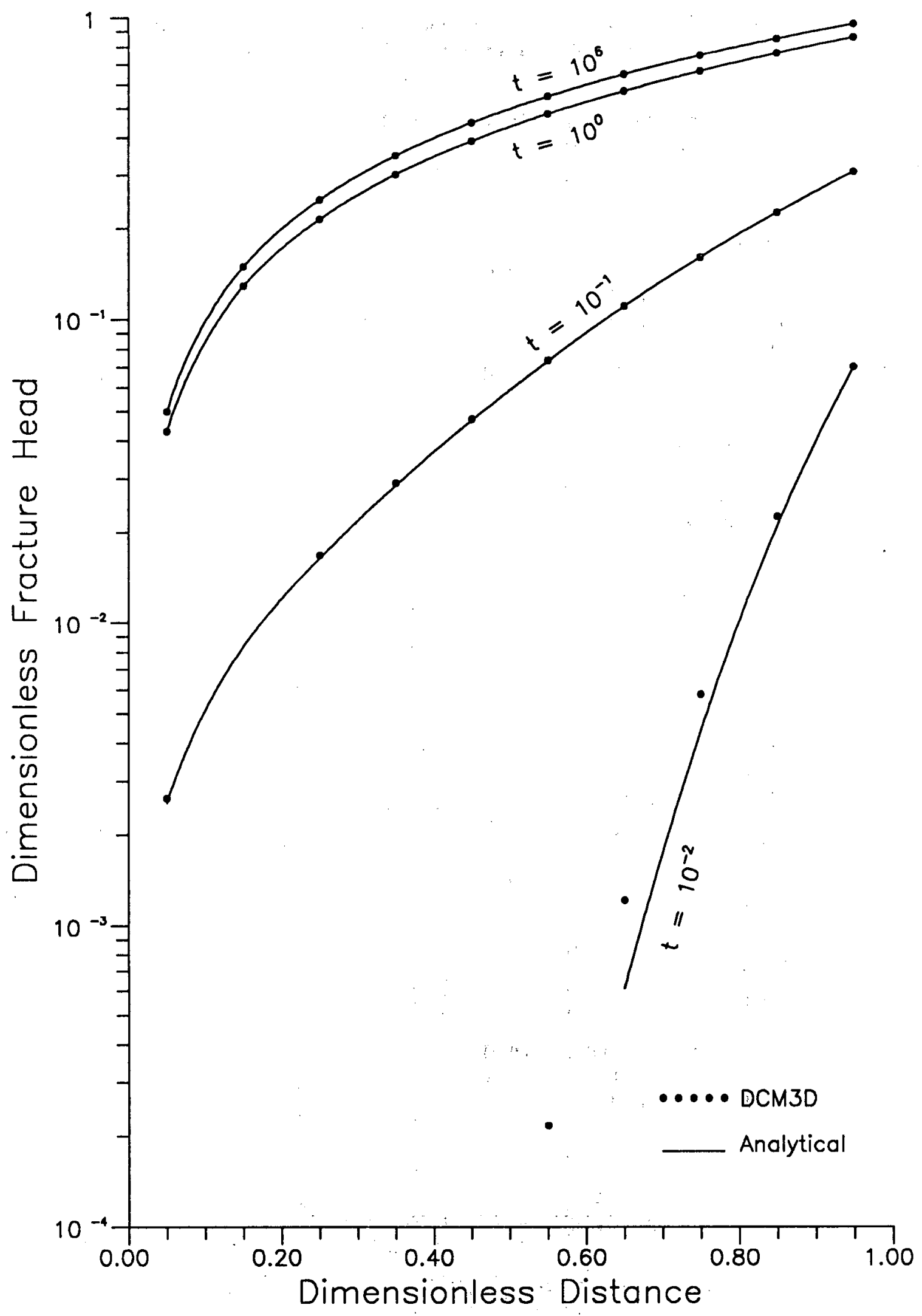

Figure 6-5: Fracture Head Results for the One-Dimensional Horizontal Saturated Matrix/Fracture Flow Problem 


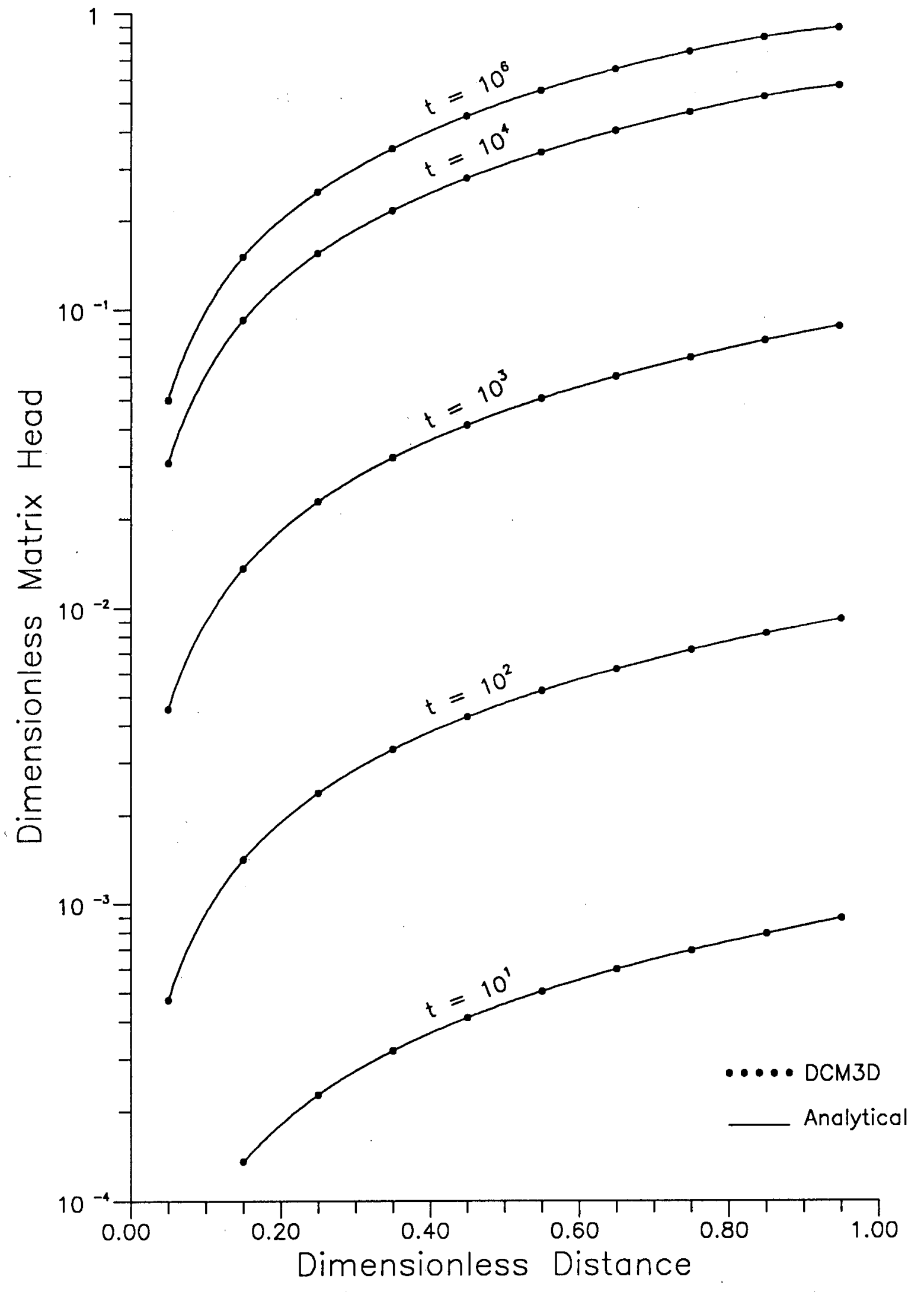

Figure 6-6: Porous Medium Head Results for the One-Dimensional Horizontal Saturated Matrix/Fracture Flow Problem 
the fractures and to test the codes ability to solve multidimensional problems. In this problem a well in an infinitely large aquifer of finite thickness is pumped at a constant rate. It is assumed that the hydraulic conductivity of the porous medium is so small compared to the hydraulic conductivity of the fractures that it can be safely neglected. However, transfer of water between the porous medium and the fractures occurs because of the transfer term. Any water removed from the pumping well comes only from the fractures. The drawdown response in the fractures is rapid. At early times the drawdown response in the porous medium is slow. At larger times the drawdown in the porous medium approaches that in the fractures. A schematic of the problem is presented in Figure 6-7.

Well flow problems are typically run as dimensionless ones as was this one. The input parameters were chosen so that the code solves for the drawdown results in terms of dimensionless head. Since this is a radially symmetric flow problem, only one quadrant of the grid was solved for. There were 1600 grid blocks used in the simulation, 40 in both the $x$ - and $y-$ directions. At the pumping well the $x$-direction grid block width was 0.01 (dimensionless). The grid block widths were expanded at a rate such that the next grid block width was 1.25209 times the width of the preceding one. The total length of the grid in the $x$-direction was 319.0. The y-direction grid block widths were expanded in the same manner. A copy of the input file is presented in Appendix B.

A comparison of the flow code porous medium and fracture drawdowns with the analytical solution from Ross et al. (1982) and Streltsova-Adams (1978) are presented in Figure 6-8. The numerically predicted fracture drawdowns agree extremely well with the analytically predicted ones. The numerically predicted fracture drawdowns are approximately 0.7 per cent higher than the analytically predicted ones for all dimensionless times except for the dimensionless time of 0.5 where the error is slightly more than 1.0 per cent. The fracture drawdown relative errors become larger for smaller times. The numerically predicted porous medium drawdowns are approximately 15 per cent higher than the analytically predicted ones for small dimensionless times of 0.2 (off the figure) and 0.7 per cent higher at large dimensionless times. However, in absolute terms, the porous medium drawdown predictions for smaller dimensionless times compare much better than those at late dimensionless times. At large dimensionless times, the numerically predicted porous medium and fracture drawdowns are almost equal but the fracture drawdowns are slightly larger than the porous medium drawdowns. Overal1, there appears to be a good agreement between the numerically and analytically produced drawdowns.

\subsection{Two-Dimensional Recharge}

The two-dimensional recharge problem is an experiment performed and modeled by Vauclin et al. (1979). The purpose of this problem is to test if the governing equations implemented in the code are capable of solving unsaturated flow problems. In this problem a $2-m$-high by $3-\mathrm{m}$-long slab of soil is recharged at a rate of $4.111 \times 10^{-5} \mathrm{~m} / \mathrm{s}$ over a $0.5-\mathrm{m}$ long region at the top left corner of the slab. The water table in the slab is initially set at $0.65 \mathrm{~m}$. The slab is impermeable on the bottom and the left side. The lower $0.65 \mathrm{~m}$ on the right side has a fixed total head of $0.65 \mathrm{~m}$. The 


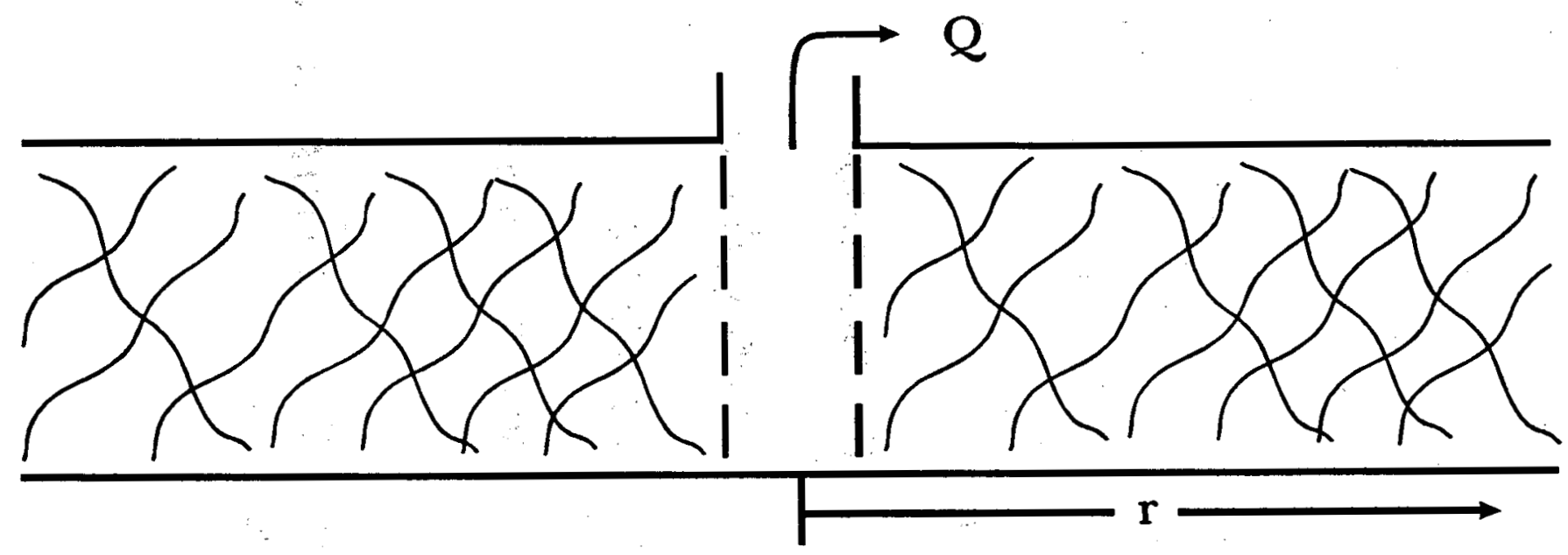

Fractures:

Matrix:

$$
\begin{array}{ll}
\mathrm{T}_{\mathrm{f}} \mathrm{r} \frac{\partial}{\partial \mathrm{r}} \mathrm{r} \frac{\partial s_{\mathrm{f}}}{\partial \mathrm{r}}=\mathrm{S}_{\mathrm{f}} \frac{\partial \mathrm{s}_{\mathrm{f}}}{\partial \mathrm{t}}-\alpha\left(\mathrm{s}_{\mathrm{m}}-\mathrm{s}_{\mathrm{f}}\right) & 0=\mathrm{S}_{\mathrm{m}} \frac{\partial \mathrm{s}_{\mathrm{m}}}{\partial \mathrm{t}}+\alpha\left(\mathrm{s}_{\mathrm{m}}-\mathrm{s}_{\mathrm{f}}\right) \\
\lim _{\mathrm{r} \rightarrow 0} \mathrm{r} \frac{\partial \mathrm{s}_{\mathrm{f}}}{\partial \mathrm{r}}=-\frac{\mathrm{Q}}{2 \pi} & \mathrm{s}_{\mathrm{m}}(\mathrm{r}, 0)=0 \\
\lim _{\mathrm{r} \rightarrow \infty} \begin{array}{l}
\mathrm{s}_{\mathrm{f}}(\mathrm{r}, \mathrm{t})=0 \\
\mathrm{~s}_{\mathrm{f}}(\mathrm{r}, 0)=0
\end{array} &
\end{array}
$$




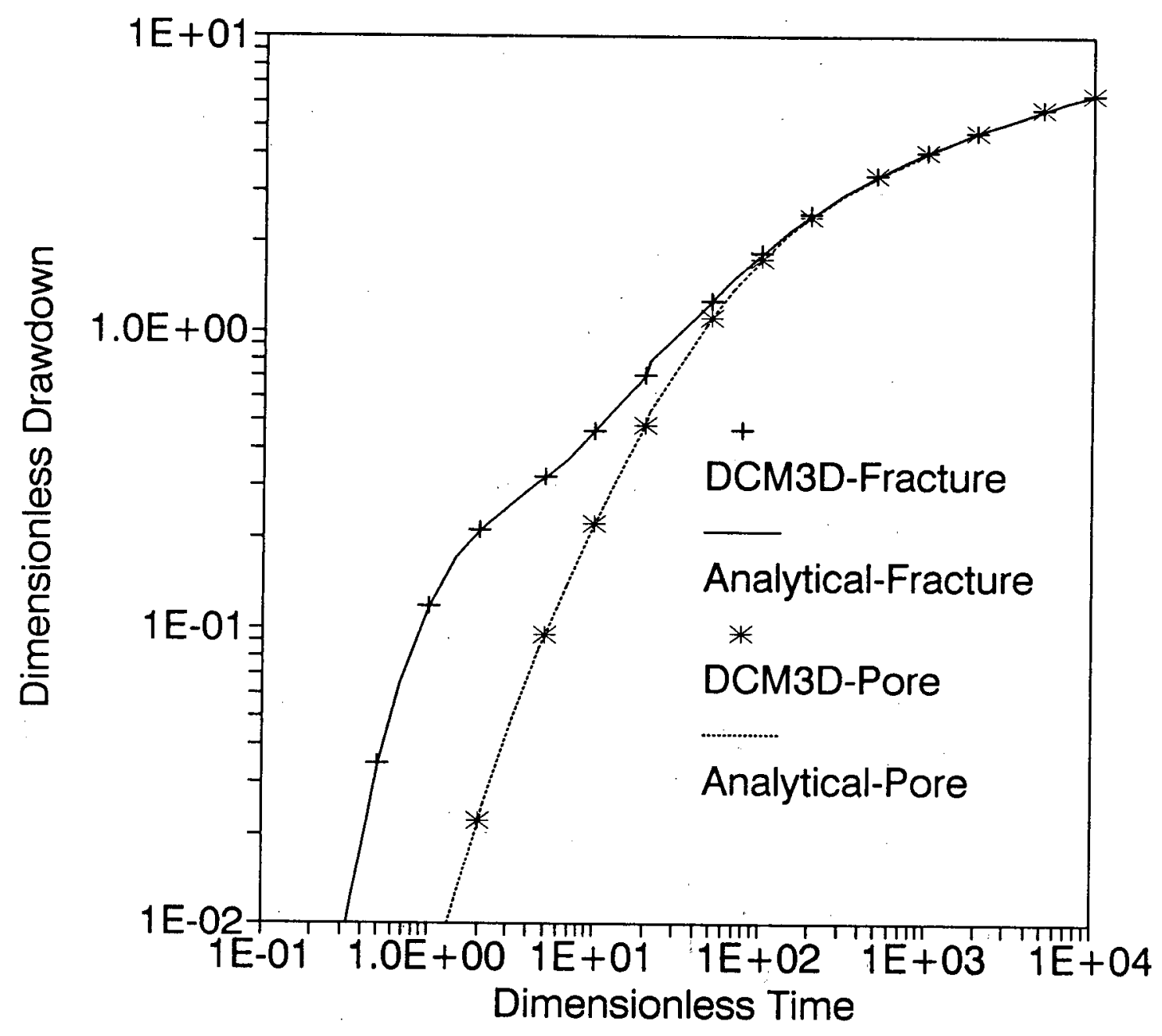

Figure 6-8: DCM3D Results for Well Flow in a Fractured Porous Medium 
remaining boundary conditions are seepage faces. The time distributions of pressure are observed at several points inside the slab. These observed pressures should be compared with those calculated by DCM3D. The hydraulic parameters and characteristic curves for the problem are presented in Vauclin et al. Figure 6-9 presents a schematic of the problem. Figure 6-10 presents the problem's characteristic curves.

The grid used in this problem consisted of 1800 grid blocks. In the vertical direction 100 grid blocks, each $0.02 \mathrm{~m}$ wide were used. In the horizontal direction 10 grid blocks, each $0.10 \mathrm{~m}$ wide were used between the left boundary and $1.0 \mathrm{~m}$ away from the boundary. Eight grid blocks, each $0.25 \mathrm{~m}$ in width were used from that point to the right boundary.

The left and bottom boundaries were treated as zero flux boundaries. The five most left grid blocks along the top boundary were each assigned a recharge rate of $4.1111 \times 10^{-5} \mathrm{~m} / \mathrm{s}$ to simulate the recharge. The remaining thirteen grid blocks, which are actually seepage faces, were assigned a zero flux boundary condition. They were assigned zero flux boundary conditions because it was believed that, since the main direction of flow is down, only minimal amount of water would flow through the boundary and because DCM 3D cannot model seepage faces. Along the right side, the bottom 33 grid blocks were assigned fixed pressure heads based on the depth of the grid block center below the water table. For instance, the grid block at the water table was assigned a pressure head of 0.0 and the bottom grid block was assigned a pressure head of 0.64 . The remaining grid blocks on the right side were assigned zero flux boundary conditions. A zero flux initial condition was used. The input file is presented in Appendix $C$.

In order to run the problem the function subprogram KREL in DCM 3D had to be modified because the hydraulic conductivity curve presented in Figure 6-10 is not the van Genuchten type. The modified function subprogram is presented in Table 6-4.

The results of the $8 \mathrm{~h}$ simulation for a vertical line $0.19 \mathrm{~m}$ from the left boundary is presented in Figure 6-11. This vertical line is under the recharge zone and shows water movement due to suction at times less than $1.5 \mathrm{~h}$ and due to water table rise for times greater than $1.5 \mathrm{~h}$. DCM3D simulates a faster movement of water for times less than $1.5 \mathrm{~h}$. The DCM $3 \mathrm{D}$ break through curve shows that the predicted water front location is deeper than for the observed one. The difference increases with time. At about $2 \mathrm{~h}$, the DCM3D results and the observed results compare favorably. At $8 \mathrm{~h}$ DCM3D predicts a faster water table rise than observed in the experiment. Overal1, DCM3D seems to predict a faster movement of water than actually observed. It appears that some heterogeneities exist, especially at $0.5 \mathrm{~m}$, that may account for this difference. 


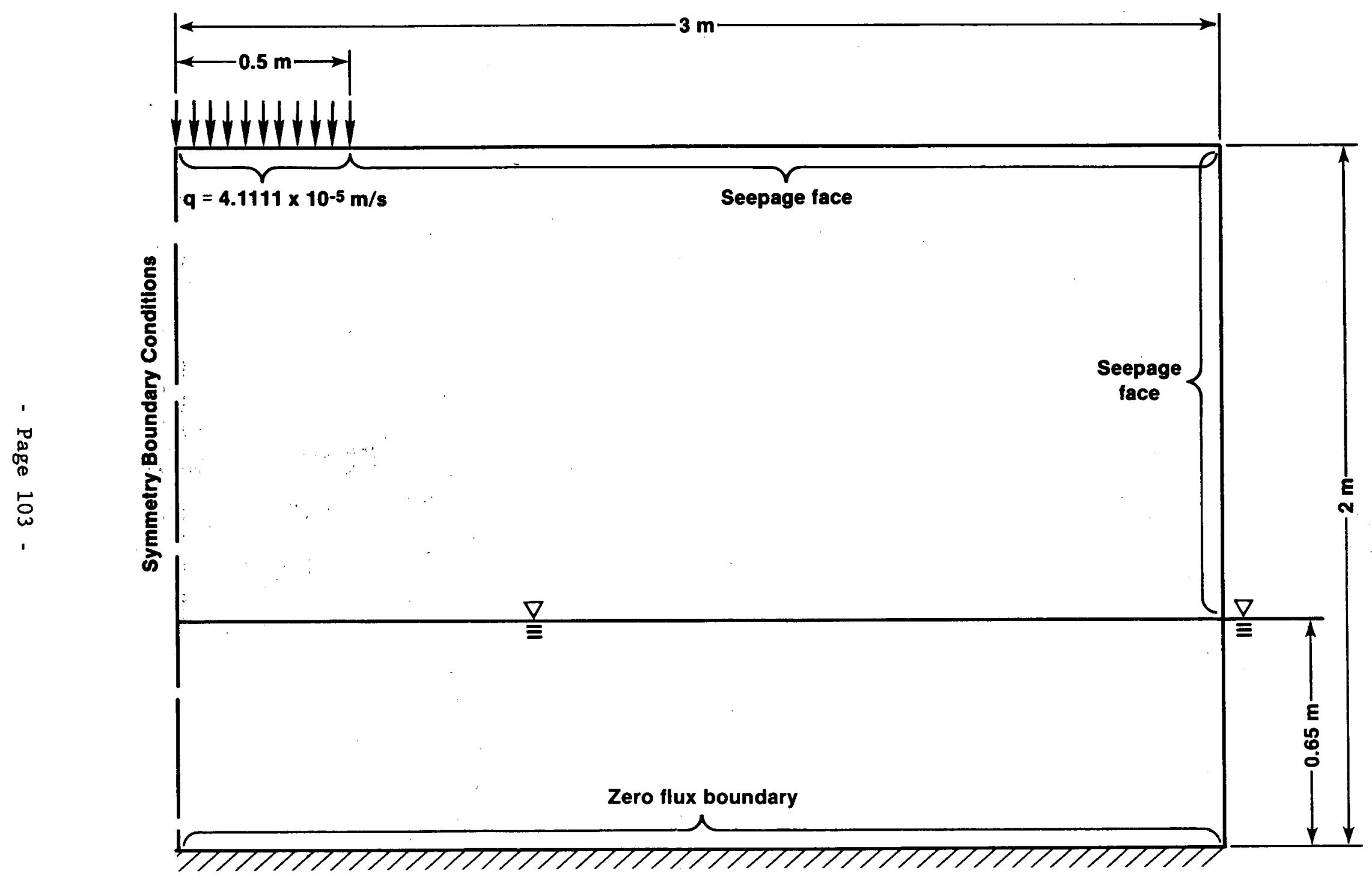

Figure 6-9: Schematic of Two-Dimensional Recharge Problem 


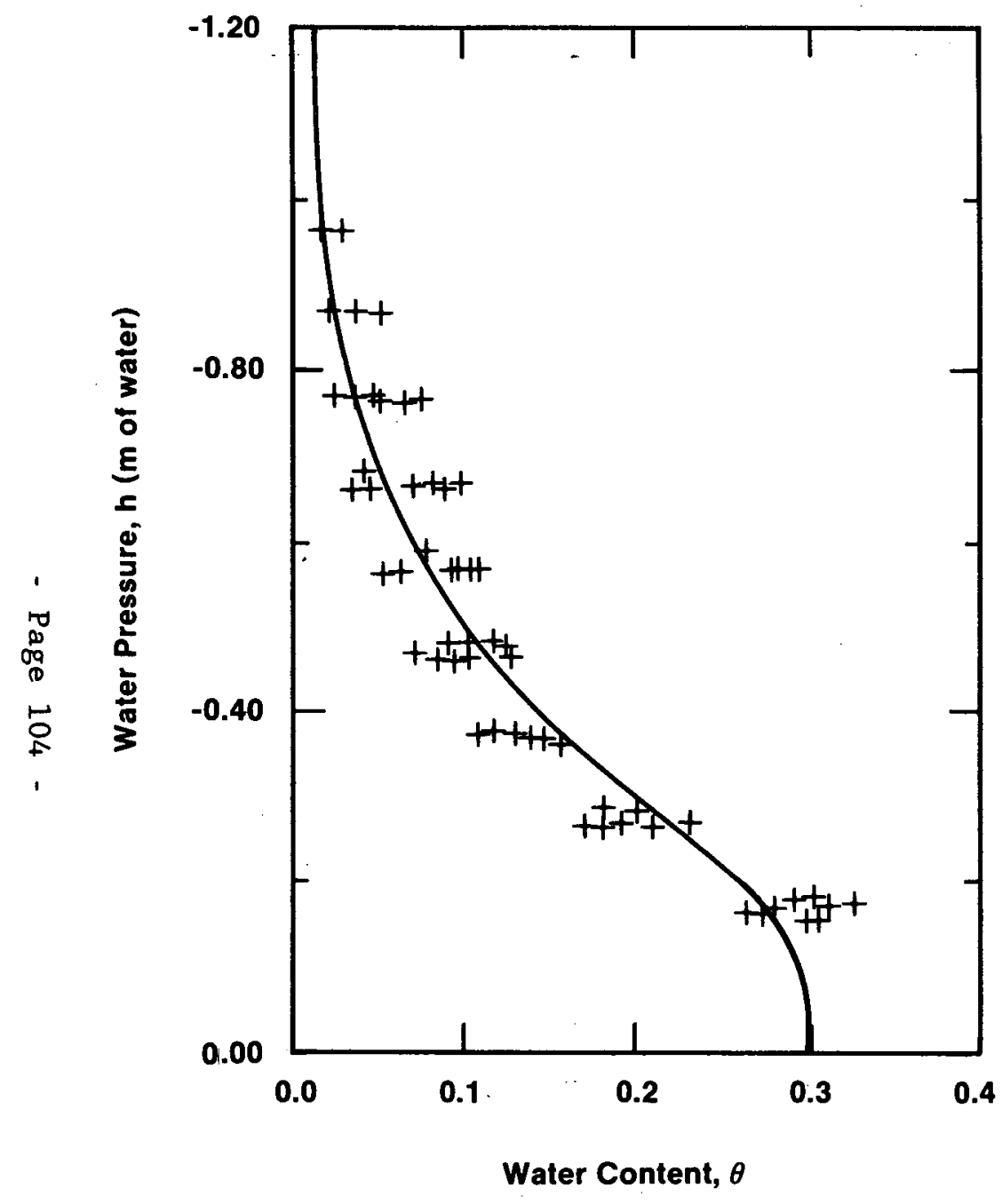

(a)

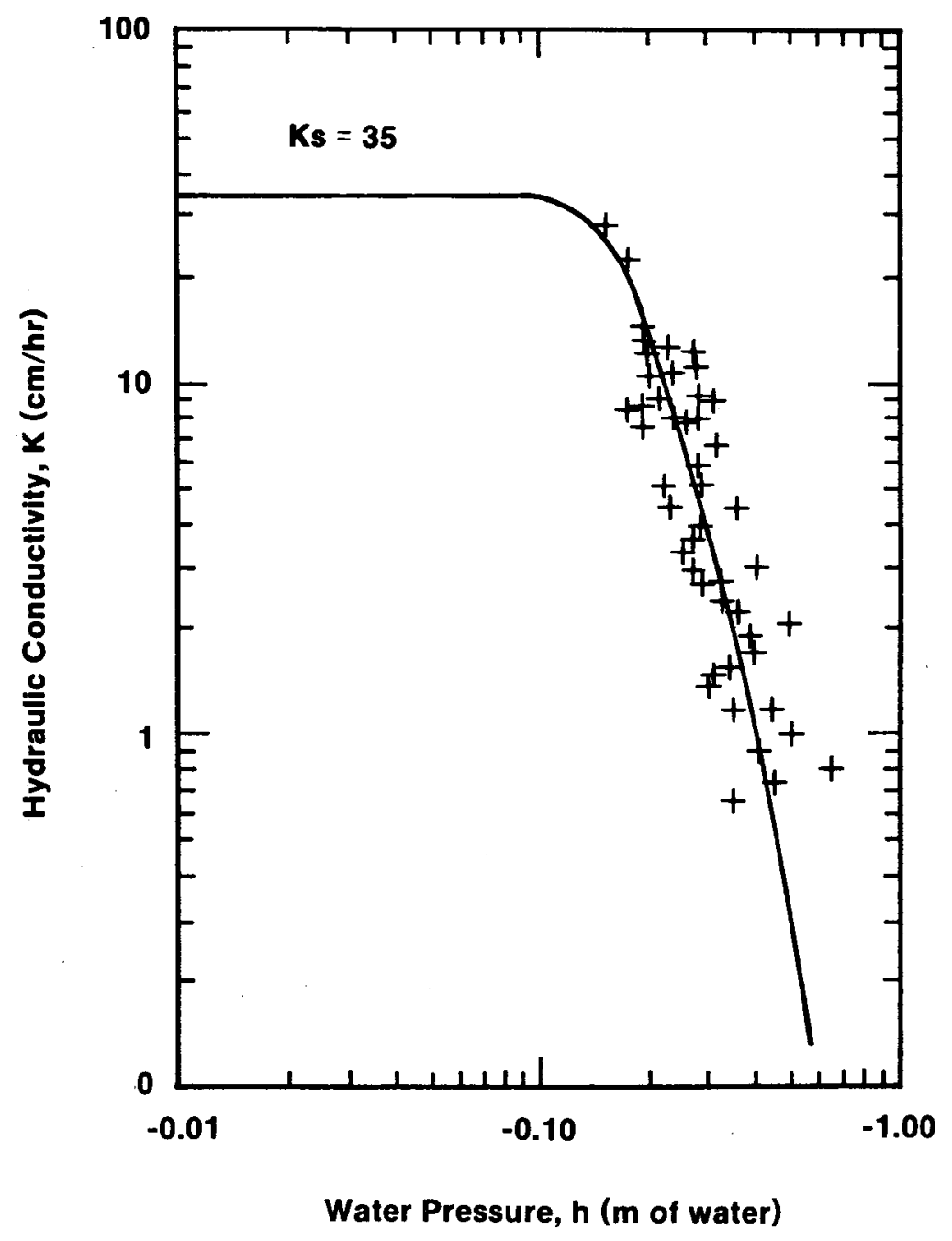

(b)

Figure 6-10: Characteristic Curves for the Two-Dimensional Recharge Problem 
Table 6-4: Hydraulic Conductivity Function Subprogram for Two-Dimensional Recharge Problem

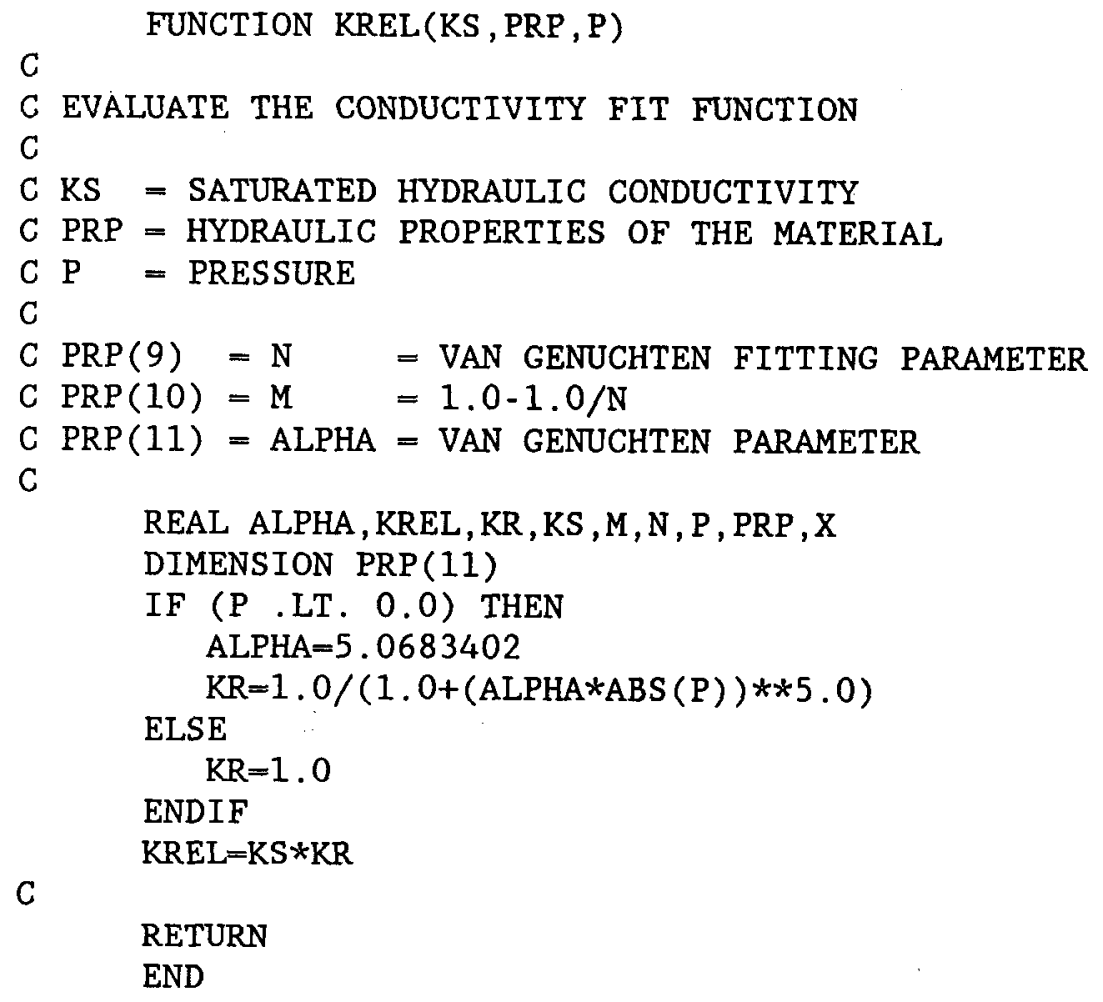




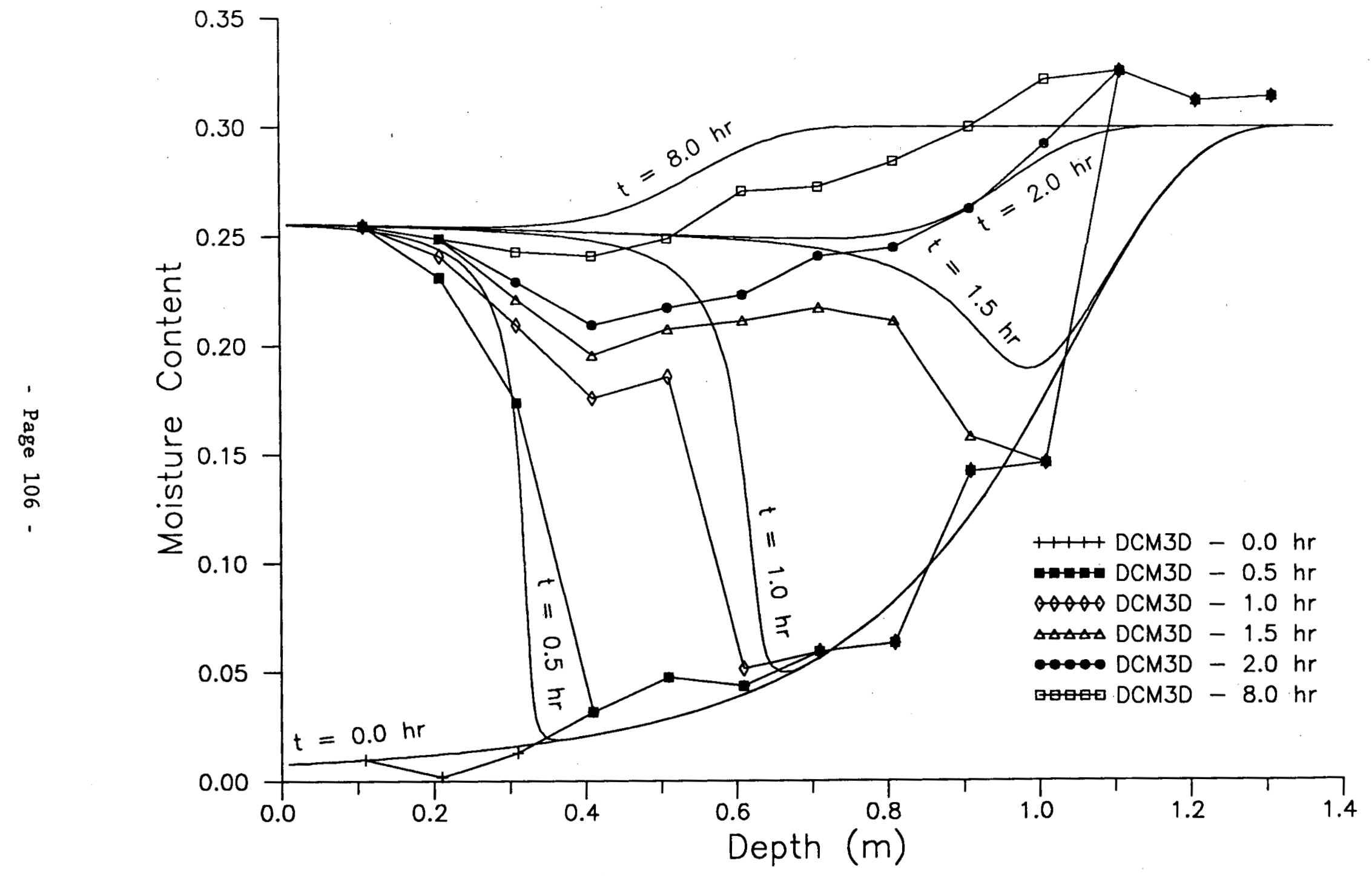

Figure 6-11: DCM3D Results for Two-Dimensional Recharge Problem 


\subsection{REFERENCES}

Bear, J., Dynamics of Fluids in Porous Media, American Elsevier Publishing Co., New York, 1972.

Bear, J., Hydraulics of Groundwater, McGraw-Hill Book Co., New York, 1979.

Bear, J. and Y. Bachmat, Introduction to Modeling of Transport Phenomena in Porous Media, Kluwer Academic Publishers, Boston, 1990.

Bird, R. B., W. E. Stewart, and E. N. Lightfoot, Transport Phenomena, John Wiley and Sons, New York, 1960.

Brown, P. N. and A. C. Hindmarsch, "Reduced Storage Matrix Methods In Stiff ODE Systems," Lawrence Livermore National Laboratory Report UCRL95088, rev. 1, Livermore, CA, June, 1987.

Department of Energy, "Environmental Assessment, Yucca Mountain Site, Nevada Research and Development Area, Nevada", U. S. Department of Energy Report DOE/RW-0073 (vol. 1), Washington DC, May 1986.

Hindmarsh, A. C., "ODEPACK, A Sytemized Collection of ODE Solvers" in Scientific Computing, R. S. Stepleman et al.,Eds., North-Holland, Amsterdam, pp. 55-64, 1983.

Klavetter, E. A., and R. R. Peters, "Fluid Flow in a Fractured Rock Mass," Sandia National Laboratories Report SAND85-0855, Albuquerque, NM, March 1986a.

Klavetter, E. A., and R. R. Peters, "Estimation of Hydrologic Properties of an Unsaturated, Fractured Rock Mass," Sandia National Laboratories Report SAND84-2642, Albuquerque, NM, July 1986b.

Martinez, M. J., "Capillary-Driven Flow in a Fracture Located in a Porous Medium," Sandia National Laboratories Report SAND84-1697, Albuquerque, NM, September 1988.

Mualem, Y., "A New Model for Predicting the Hydraulic Conductivity of Unsaturated Porous Media," Water Resources Research 12(3), 513-522 (1976).

Philip, J. R., "Numerical Solution of Equations of the Diffusive Type with Diffusivity Concentration-Dependent," Transactions, Faraday Society $51,885-892$ (1955).

Press, W. H., B. P. Flannery, S. A. Teukolsky, and W. T. Flannery, Numerical Recipes. The Art of Scientific Computing, Cambridge University Press, New York, 1986.

Pruess, K., Lawrence Berkeley Laboratory, "TOUGH Users Guide," U. S. Nuclear Regulatory Commission Report NUREG/CR-4645, Washington, DC, August 1987. 
Ross, B., J. W. Mercer, S. D. Thomas, and B. H. Lester, Geotrans, Inc., "Benchmark Problems for Repository Siting Models," U. S. Nuclear Regulatory Commission Report NUREG/CR-4766, Washington, DC, December 1982 .

Sneddon, I. N., The Use of Integral Transforms, McGraw-H111 Book Co., New York, 1972 .

Streltsova-Adams, T. D. "We1l Hydraulics In Heterogeneous Aquifer Formations," in Advances in Hydroscience, V. T. Chow, Ed. (Academic Press, New York, 1978), pp. 357-423.

Travis, B. J., "TRACR3D: A Model of Flow and Transport in Porous/Fractured Media," Los Alamos Report LA-9667-MS, Los Alamos, NM, May 1984.

Travis, B. J., S. W. Hodson, H. E. Nuttall, T. L. Cook, and R. S. Rundberg, "Preliminary Estimates of Water Flow and Radionuclide Transport in Yucca Mountain," Material Research Society Symposium Proceedings 26, 1039-1047 (1984).

Updegraff, C. D., GRAM, Inc., "Comparison of Strongly Heat-Driven Flow Codes for Unsaturated Media," U. S . Nuclear Regulatory Commission Report NUREG/CR-5367, Washington, DC, August 1989.

van Genuchten, M. Th., "A Closed-form Equation For Predicting the Hydraulic Conductivity of Unsaturated Soils," Soil Science 44(5), 892-898 (1980).

van Golf-Racht, T. D., Fundamentals of Fractured Reservoir Engineering, Elsevier/North Holland Inc., New York, 1982.

Vauclin, M., D. Khanji, and G. Vachaud, "Experimental and Numerical of a Transient Two-Dimensional Unsaturated-Saturated Water Table Recharge Problem," Water Resources Research 15(5), 1089-1101 (1979).

Wang, J. S. Y., and T. N. Narasimhan, Lawrence Berkeley Laboratory, "Hydrologic Mechanisms Governing Partially Saturated Flow in Fractured Welded Units and Porous Non-welded Units at Yucca Mountain," Sandia National Laboratories Contractor Report SAND857114, Albuquerque, NM, October 1986. 


\subsection{INTRODUCTION}

A one-dimensional flow model benchmark was formulated and solved for the coupled matrix-fracture saturated flow initial value problem. The initial pressure head was assumed to be zero in both the matrix and fracture. The left side boundary conditions were set at zero head for both the matrix and the fracture. The right side boundary conditions used were a fixed flux, $\epsilon$, into the fracture and a zero flux into the matrix. The hydraulic conductivity and the specific storage were assumed to be constants, independent of the pressure head for both the matrix and fracture.

\subsection{THEORY}

The coupled matrix and fracture equations for flow in a one-dimensional, saturated, fractured, porous medium are:

$$
S_{\mathrm{s}}^{\mathrm{m}} \frac{\partial h^{\mathrm{m}}}{\partial t}=K^{\mathrm{m}} \frac{\partial^{2} h^{\mathrm{m}}}{\partial \mathrm{x}^{2}}+\Gamma\left(h^{\mathrm{f}}-h^{\mathrm{m}}\right)
$$

and

$$
S_{\mathrm{g}}^{\mathrm{f}} \frac{\partial h^{\mathrm{f}}}{\partial t}=K^{\mathrm{f}} \frac{\partial^{2} h^{\mathrm{f}}}{\partial x^{2}}-\Gamma\left(h^{\mathrm{f}}-h^{\mathrm{m}}\right)
$$

where $K^{\mathrm{m}}$ and $K^{\mathrm{f}}$ are the hydraulic conductivities of the matrix and fracture continuum, respectively; $S_{\mathrm{s}}{ }^{\mathrm{m}}$ and $S_{\mathrm{s}}{ }^{\mathrm{f}}$ are the specific storage coefficients of the matrix and fracture continuum, respectively; $h^{m}$ and $h^{f}$ are the total heads in the matrix and fracture continuum, respectively; $\Gamma$ is the matrixfracture transfer coefficient; $t$ is the time; and $x$ is the distance. The domain of $x$ is $0 \leq x \leq L$, where $L$ is the length of the fractured, porous medium.

The initial conditions are:

$$
h^{m}(x, t=0)=0
$$

and

$$
h^{f}(x, t=0)=0
$$

The left side boundary conditions are: 


$$
h^{m}(x=0, t)=0
$$

and

$$
h^{\mathrm{f}}(\mathrm{x}=0, t)=0
$$

The right side boundary conditions are:

$$
\frac{\partial h^{\mathrm{m}}(x=L, t)}{\partial x}=0
$$

and

$$
\frac{\partial h^{f}(x=L, t)}{\partial x}=\frac{\epsilon}{K^{f}}
$$

In dimensionless form, Eq. A-1 through Eq. A-8 become, respectively:

$$
\begin{gathered}
\sigma \frac{\partial h^{\mathrm{m}^{\prime}}}{\partial t^{\prime}}=\kappa \frac{\partial^{2} h^{\mathrm{m}^{\prime}}}{\partial \mathbf{x}^{\prime 2}}+\gamma\left(h^{\mathrm{f}^{\prime}}-h^{\mathrm{m}^{\prime}}\right) \\
\frac{\partial h^{\mathrm{f}^{\prime}}}{\partial t^{\prime}}=\frac{\partial^{2} h^{\mathbf{f}^{\prime}}}{\partial \mathrm{x}^{\prime 2}}-\gamma\left(h^{\mathrm{f}^{\prime}}-h^{\mathrm{m}^{\prime}}\right) \\
h^{\mathrm{m}^{\prime}}\left(\mathrm{x}^{\prime}, t^{\prime}=0\right)=0 \\
h^{\mathrm{f}^{\prime}}\left(\mathrm{x}^{\prime}, t^{\prime}=0\right)=0 \\
h^{\mathrm{m}^{\prime}}\left(\mathrm{x}^{\prime}=0, t^{\prime}\right)=0 \\
h^{\mathrm{f}^{\prime}}\left(\mathrm{x}^{\prime}=0, t^{\prime}\right)=0 \\
\frac{\partial h^{\mathrm{m}^{\prime}}\left(\mathrm{x}^{\prime}=1, t^{\prime}\right)}{\partial \mathrm{x}^{\prime}}=0
\end{gathered}
$$

and 


$$
\frac{\partial h^{f^{\prime}}\left(x^{\prime}=1, t^{\prime}\right)}{\partial x^{\prime}}=1
$$

where

$$
\begin{aligned}
& x^{\prime}=x / L, t^{\prime}=K^{\mathrm{f}} t / S^{\mathrm{f}} L^{2}, \gamma=\Gamma L^{2} / K^{\mathrm{f}}, \kappa=K^{\mathrm{m}} / K^{\mathrm{f}}, \sigma=S^{\mathrm{m}} / S^{\mathrm{f}}, \\
& h^{\mathrm{m}}=K^{\mathrm{f}} h^{\mathrm{m}} / \epsilon L, \text { and } h^{f \prime}=K^{\mathrm{f}} h^{\mathrm{f}} / \epsilon L .
\end{aligned}
$$

\subsection{SOLUTION}

This problem was solved analytically by performing a double transform: a Laplace transform on the time variable $(t ; p)$ and a Generalized Sine transform (Sneddon, 1972) on the spatial variable $(x ; \zeta)$. Performing the double transform on the Eq. A-10 and Eq. A-11, apllying the initial and boundary conditions, and solving for the double transformed variables, $H^{\mathrm{m}}$ and $H^{f}$, results in:

$$
H^{\mathrm{m}}=(-1)^{\mathrm{n}+1} \frac{\gamma / \sigma}{p\left[(p+a)(p+b)-\gamma^{2} / \sigma\right]}
$$

and

$$
H^{\mathrm{f}}=(-1)^{\mathrm{n}+1} \frac{p+a}{p\left[(p+a)(p+b)-\gamma^{2} / \sigma\right]}
$$

where $a=\left(\gamma+\kappa \zeta^{2}\right) / \sigma, b=(\gamma+\zeta)^{2}$, and $\zeta=(2 n-1) \pi / 2$ for $n=1,2,3, \ldots$. $\kappa, \sigma, \zeta$, and $\gamma$ are all positive.

Inversion of the Laplace and Generalized Sine transform variables, $H^{\mathrm{m}}$ and $H^{f}$ yields the following time dependent solutions, which are broken down into a steady-state part and a transient part. The steady state part of the solutions are:

$$
h_{\mathrm{s}}^{\mathrm{f}^{\prime}}=\frac{1}{1+\kappa}\left[x+\frac{\kappa}{\sqrt{\gamma\left(1+\frac{1}{\kappa}\right)}} \frac{\sinh \left(x \sqrt{\gamma\left(1+\frac{1}{\kappa}\right)}\right)}{\cosh \left(\sqrt{\gamma\left(1+\frac{1}{\kappa}\right)}\right)}\right.
$$

and

$$
h_{\mathrm{s}}^{\mathfrak{m}^{\prime}}=\frac{\mathrm{x}-h_{\mathrm{s}}^{\mathbf{f}^{\prime}}}{\kappa}
$$


The transient parts of the solutions are:

$$
\begin{aligned}
h_{\mathrm{t}}^{\mathrm{m}^{\prime}}=\frac{\gamma}{\sigma} \sum_{\mathrm{n}=1}^{\infty} \frac{(-1)^{\mathrm{n}+1}}{\beta} & {\left[\frac{\exp (-(\alpha+\beta) t)}{\alpha+\beta}-\frac{\exp (-(\alpha-\beta) t)}{\alpha-\beta}\right] \times } \\
& \sin \left(\frac{(2 n-1) \pi \mathrm{x}}{2}\right)
\end{aligned}
$$

and

$$
\begin{aligned}
h_{\mathrm{t}}^{f^{\prime}}=\frac{1}{\beta} \sum_{n=1}^{\infty}(-1)^{\mathrm{n}+1}\left[\left(\frac{a}{\alpha+\beta}-1\right) \exp (-(\alpha+\beta) t)-\left(\frac{a}{\alpha-\beta}-1\right) \exp (-(\alpha-\beta) t)\right] \times \\
\quad \sin \left(\frac{(2 n-1) \pi x}{2}\right)
\end{aligned}
$$

where

$$
\alpha=(a+b) / 2, \beta=\left[(a-b)^{2}+4 \gamma^{2} / \sigma\right]^{1 / 2} / 2, \text { and } \alpha^{2}-\beta^{2}=a b-\gamma^{2} / \sigma>0 .
$$

The condition $\alpha^{2}-\beta^{2}>0$ precludes a pole in the time dependent solution at $\alpha=\beta$. This condition is always satisfied provided that $\kappa, \sigma, \zeta$, and $\gamma$ are all greater than zero. This analytic solution was programmed and evaluated in double precision.

\subsection{RESULTS}

The numerical solution for testing with the analytical benchmark solution was performed with 10 spatial grid cells. "The input deck is listed in Table A-1. The input parameters correspond to the dimensionless parameters $\kappa=10^{-3}, \sigma=10^{3}$, and $\gamma=0.1$. The output from the analytical benchmark evaluation and the numerical code was generated for relevant dimensionless times. The results are summarized in Table A-2.

Examination of Table A-2 indicates that for dimensionless times $t^{\prime} \geq 1.0$ and all distances, $0.05 \leq \mathrm{x}^{\prime} \leq 0.95$, the matrix and fracture numerical solutions agree with the analytical solutions to two digits (or better) for all $x^{\prime}$ and $t^{\prime}$. At the earliest time $\left(t^{\prime}=10^{-2}\right)$, the evaluated analytic solution for the matrix did not converge to a non-negative result (even with 40000 summation terms) for $x^{\prime}<0.5$. For $x^{\prime}>0.5$ the analytical and numerical solutions are not in particularly good agreement. However, for the range $0.01<t^{\prime} \leq 1.0$, the analytic and numerical solutions are within about $10 \%$ agreement even for the worst case. For $t^{\prime}>1.0$, the analytical and numerical solutions are in quite good agreement for all $x^{\prime}$ and $t^{\prime}$.

The overall good agreement of the numerical code with these benchmark results implies proper execution and functioning of the numerical code. The parameters used were considered representative for anticipated applications and for the relative equation 'stiffness' expected to be encountered in such problems. 
Table A-1: Input Deck for Numerical Calculation

1-D, SATURATED, FRACTURED, POROUS MEDIA TEST PROBLEM

KAPPA-1000.0, SIGMA-0.001, GAMMA-0.1

C. DAVID UPDEGRAFF

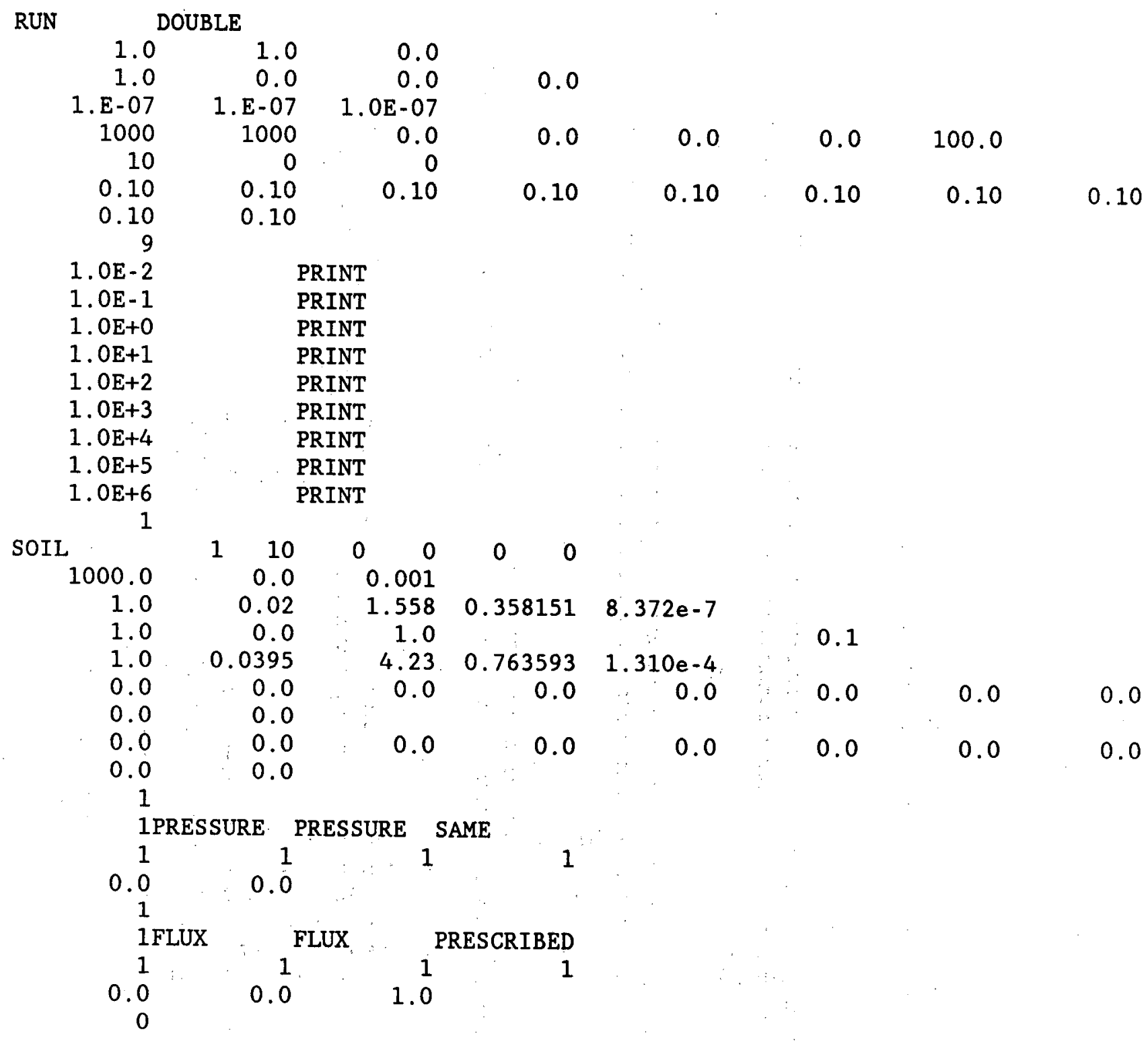


Table A-2: Analytical and Numerical Comparison Results

$$
\kappa=0.001, \sigma=1000 ., \gamma=0.1
$$

Analytical

$t^{\prime}$

1. $0 \mathrm{E}-02$

$1.0 \mathrm{E}-02$

1. OE-02

1. OE-02

1. $0 \mathrm{E}-02$

1. $0 \mathrm{E}-02$

1. OE-02

1. $0 \mathrm{E}-02$

1. $0 \mathrm{E}-02$

1. $0 \mathrm{E}-02$

$1.0 \mathrm{E}-01$

1. $0 \mathrm{E}-01$

1. $0 E-01$

1. $0 \mathrm{E}-01$

1. $O E-01$

1. $0 \mathrm{E}-01$

1. $0 E-01$

1. $0 \mathrm{E}-01$

1. $0 \mathrm{E}-01$

1. 0 E-01

1. $0 \mathrm{E}+00$

1. $0 \mathrm{E}+00$

1. $0 \mathrm{E}+00$

1. $0 \mathrm{E}+00$

1. $0 \mathrm{E}+00$

1. $0 \mathrm{E}+00$

1. $0 \mathrm{E}+00$

1. $0 \mathrm{E}+00$

1. $0 \mathrm{E}+00$

1. $0 \mathrm{E}+00$

1. $0 \mathrm{E}+01$

1. $0 \mathrm{E}+01$

1. $0 \mathrm{E}+01$

1. $0 \mathrm{E}+01$

1. $0 \mathrm{E}+01$

1. $0 \mathrm{E}+01$

1. $0 \mathrm{E}+01$

1. $0 \mathrm{E}+01$

1. $0 \mathrm{E}+01$

1. $0 \mathrm{E}+01$
0.05

0.15

0.25

0.35

0.45

0.55

0.65

0.75

0.85

0.95

0.05

0.15

0.25

0.35

0.45

0.55

0.65

0.75

0.85

0.95

0.05

0.15

0.25

0.35

0.45

0.55

0.65

0.75

0.85

0.95

0.05

0.15

0.25

0.35

0.45

0.55

0.65

0.75

0.85

0.95 $h^{f \prime}$

4. $560942 E-13$

4. $130800 \mathrm{E}-11$

2. 844360E-09

1. $219820 \mathrm{E}-07$

3. $282690 \mathrm{E}-06$

5.596041E-05

6.120762E-04

4. $373821 \mathrm{E}-03$

2. $095288 \mathrm{E}-02$

$6.978531 \mathrm{E}-02$

2. 545447E-03

8.372853E-03

1. $647928 \mathrm{E}-02$

2.859827E-02

4. $672550 \mathrm{E}-02$

7. $311359 \mathrm{E}-02$

1. 101773E-01

$1.603149 \mathrm{E}-01$

2. 256705E-01

3. 078854E-01

4. 291217E-02

1. $288996 \mathrm{E}-01$

2. $153735 \mathrm{E}-01$

3. $026489 \mathrm{E}-01$

3. $910273 \mathrm{E}-01$

4. 807915E-01

5.722011E-01

6. 654890E-01

$7.608575 \mathrm{E}-01$

8. 584762E-01

4.760419E-02

1. $428601 \mathrm{E}-01$

2. $382588 \mathrm{E}-01$

3. $338956 \mathrm{E}-01$

4. $298659 \mathrm{E}-01$

5. $262658 \mathrm{E}-01$

6. 231916E-01

7.207400E-01

8. 190085E-01

9. 180953E-01
$-3.222173 \mathrm{E}-14$

$-2.510962 \mathrm{E}-13$

$-5.581512 \mathrm{E}-13$

$-7.454362 \mathrm{E}-13$

$-6.771489 \mathrm{E}-13$

$5.992798 \mathrm{E}-12$

$1.030392 \mathrm{E}-10$

1. $051079 \mathrm{E}-09$

7.393325E-09

3. $738014 \mathrm{E}-08$

$5.702070 \mathrm{E}-09$

1. $971849 \mathrm{E}-08$

4. $231719 \mathrm{E}-08$

8.177222E-08

1. $504091 \mathrm{E}-07$

2. 666138E-07

4. 570942E-07

7. $593087 \mathrm{E}-07$

1. $223880 \mathrm{E}-06$

1. $916772 \mathrm{E}-06$

2. 640960E-06

7. $968449 \mathrm{E}-06$

1. 343284E-05

1. $912612 \mathrm{E}-05$

2. 514128E-05

3. $157272 \mathrm{E}-05$

3. 851668E-05

4. 607153E-05

5. $433812 \mathrm{E}-05$

$6.342011 \mathrm{E}-05$

4.527970E-05

1. 359319E-04

2. 268628E-04

3. 182585E-04

4. 103059E-04

5. 031931E-04

5. $971091 E-04$

6. $922446 \mathrm{E}-04$

7. 887923E-04

8. $869469 \mathrm{E}-04$
Numerical

$h^{f \prime}$

$\mathrm{h}^{\mathrm{m} \prime}$

4. 9055 E- 09

5. 5549E-08

5. 2154E-07

4.3992E-06

3. 2962E-05

2.1602E-04

1. $2180 E-03$

$5.7833 \mathrm{E}-03$

2. $2545 \mathrm{E}-02$

7.0150E-02

5. 2077E-16

$6.4683 \mathrm{E}-15$

$6.7972 \mathrm{E}-14$

$6.5109 \mathrm{E}-13$

5. $6341 \mathrm{E}-12$

4. 3574E-11

$2.9758 \mathrm{E}-10$

1. $7699 \mathrm{E}-09$

$9.0272 \mathrm{E}-09$

3. 8839 E- 08

2. 6352E-03

6.1970E-09

8.6308E-03

2. $1233 \mathrm{E}-08$

$1.6872 \mathrm{E}-02$

2. $9072 \mathrm{E}-02$

4.7214E-02

7. 3550E-02

1. $1051 \mathrm{E}-01$

1. 6052E-01

2. $2575 \mathrm{E}-01$

3.0789E-01

4. 4920E-08

8. 5524E-08

1. 5529E-07

2. 7242E-07

$4.6337 \mathrm{E}-07$

7.6529E-07

1. $2285 \mathrm{E}-06$

$1.9187 \mathrm{E}-06$

4.2892E-02

2. $6404 \mathrm{E}-06$

1. $2884 \mathrm{E}-01$

7. $9666 \mathrm{E}-06$

2. 1527E-01

$1.3430 \mathrm{E}-05$

3. $0251 E-01$

3. $9086 \mathrm{E}-01$

1. $9121 \mathrm{E}-05$

4. 8059E-01

5. 7197E-01

6.6524E-01

$7.6060 \mathrm{E}-01$

8. 5820E-01

2. 5135E-05

3. 1564E-05

3. $8505 \mathrm{E}-05$

4. $6057 \mathrm{E}-05$

5. 4320E-05

6. 3398E-05

4.7602E-02

4. 5276E-05

$1.4285 \mathrm{E}-01$

2. $3825 \mathrm{E}-01$

3. 3388E-01

4. 2985E-01

5. 2625E-01

6.2317E-01

7.2071E-01

8. 1898E-01

9.1806E-01
1. 3592E-04

2. $2685 \mathrm{E}-04$

3. $1824 \mathrm{E}-04$

4. 1028E-04

5. 0316E-04

5. $9706 \mathrm{E}-04$

6. $9219 \mathrm{E}-04$

7. $8873 \mathrm{E}-04$

8. 8683E-04 
Table A-2 (continued):

\begin{tabular}{|c|c|c|c|c|c|}
\hline 1. $0 \mathrm{E}+02$ & 0.05 & $4.762480 \mathrm{E} \div 02$ & $4.714848 \mathrm{E}-04$ & $4.7623 E-02$ & $4.7147 \mathrm{E}-04$ \\
\hline $1.0 \mathrm{E}+02$ & 0.15 & $1.429215 \mathrm{E}-01$ & $1.414971 \mathrm{E}-03$ & $1.4292 \mathrm{E}-01$ & $1.4149 \mathrm{E}-03$ \\
\hline 1. $0 \mathrm{E}+02$ & 0.25 & $2.383598 \mathrm{E}-01$ & $2.360006 \mathrm{E}-03$ & $2.3835 \mathrm{E}-01$ & $2.3599 E-03$ \\
\hline 1. $0 \mathrm{E}+02$ & 0.35 & 3. $340341 E-01$ & $3.307627 E-03$ & 3. $3402 E-01$ & $3.3075 E-03$ \\
\hline $1.0 \mathrm{E}+02$ & 0.45 & 4. $300392 E-01$ & $4.258869 \mathrm{E}-03$ & $4.3002 E-01$ & $4.2587 \mathrm{E}-03$ \\
\hline 1. $\mathrm{OE}+02$ & 0.55 & $5.264701 \mathrm{E}-01$ & $5.214775 \mathrm{E}-03$ & $5.2645 \mathrm{E}-01$ & $5.2146 \mathrm{E}-03$ \\
\hline $1.0 \mathrm{E}+02$ & 0.65 & $6.234224 \mathrm{E}-01$ & $6.176392 E-03$ & $6.2340 \mathrm{E}-01$ & $6.1762 \mathrm{E}-03$ \\
\hline 1. $0 E+02$ & 0.75 & 7.209919E-01 & $7.144773 E-03$ & $7.2096 \mathrm{E}-01$ & $7.1445 E-03$ \\
\hline 1. $0 \mathrm{E}+02$ & 0.85 & $8.192754 \mathrm{E}-01$ & $8.120978 \mathrm{E}-03$ & 8. 1924E-01 & $8.1207 \mathrm{E}-03$ \\
\hline 1. $0 \mathrm{E}+02$ & 0.95 & $9.183702 \mathrm{E}-01$ & $9.106073 \mathrm{E}-03$ & $9.1833 \mathrm{E}-01$ & $9.1008 \mathrm{E}-03$ \\
\hline $1.0 \mathrm{E}+03$ & 0.05 & $4.782117 \mathrm{E}-02$ & $4.538834 \mathrm{E}-03$ & $4.7820 \mathrm{E}-02$ & $4.5387 \mathrm{E}-03$ \\
\hline 1. $0 \mathrm{E}+03$ & 0.15 & $1.435068 \mathrm{E}-01$ & $1.362086 \mathrm{E}-02$ & $1.4350 \mathrm{E}-01$ & 1. $3620 \mathrm{E}-02$ \\
\hline $1.0 \mathrm{E}+03$ & 0.25 & $2.393223 E-01$ & $2.271597 \mathrm{E}-02$ & $2.3931 E-01$ & $2.2715 E-02$ \\
\hline 1. $0 \mathrm{E}+03$ & 0.35 & $3.353545 \mathrm{E}-01$ & 3.183290E-02 & $3.3534 \mathrm{E}-01$ & $3.1832 \mathrm{E}-02$ \\
\hline $1.0 \mathrm{E}+03$ & 0.45 & 4. $316902 E-01$ & $4.098040 \mathrm{E}-02$ & 4. $3168 \mathrm{E}-01$ & $4.0979 E-02$ \\
\hline $1.0 \mathrm{E}+03$ & 0.55 & $5.284167 \mathrm{E}-01$ & $5.016727 \mathrm{E}-02$ & $5.2840 E-01$ & $5.0166 \mathrm{E}-02$ \\
\hline 1. $0 \mathrm{E}+03$ & 0.65 & $6.256215 \mathrm{E}-01$ & $5.940232 \mathrm{E}-02$ & $6.2560 \mathrm{E}-01$ & $5.9400 \mathrm{E}-02$ \\
\hline 1. $0 E+03$ & 0.75 & 7.233926E-01 & $6.869443 E-02$ & $7.2337 \mathrm{E}-01$ & $6.8692 \mathrm{E}-02$ \\
\hline 1. $0 \mathrm{E}+03$ & 0.85 & $8.218185 \mathrm{E}-01$ & $7.805242 \mathrm{E}-02$ & $8.2179 \mathrm{E}-01$ & $7.8035 \mathrm{E}-02$ \\
\hline 1. $0 \mathrm{E}+03$ & 0.95 & 9.209882E-01 & $8.729808 \mathrm{E}-02$ & $9.2096 \mathrm{E}-01$ & $8.7029 E-02$ \\
\hline 1. $0 E+04$ & & $4.906940 \mathrm{E}-02$ & $3.071025 \mathrm{E}-02$ & E-02 & LE -02 \\
\hline 1. $0 E+04$ & 0.15 & $1.472265 \mathrm{E}-01$ & $9.214871 \mathrm{E}-02$ & $1.4722 \mathrm{E}-01$ & $9.2147 \mathrm{E}-02$ \\
\hline $1.0 E+04$ & 0.25 & $2.454388 \mathrm{E}-01$ & 1.5364 & $2.4543 E-01$ & 1. $5364 \mathrm{E}-01$ \\
\hline $1.0 \mathrm{E}+04$ & 0.35 & $3.437428 \mathrm{E}-01$ & $2 E-01$ & $4 E-01$ & $522 \mathrm{E}-01$ \\
\hline $1.0 \mathrm{E}+04$ & 0.45 & 4.4 & 2.76 & $7 E-01$ & $2.7692 \mathrm{E}-01$ \\
\hline 1. $0 \mathrm{E}+04$ & 0.55 & $32 E-01$ & 3. 387984E- 01 & $5.4076 \mathrm{E}-01$ & $3.3877 \mathrm{E}-01$ \\
\hline $1.0 E+04$ & 0.6 & $730 E-01$ & $4.008251 E-01$ & $6.3956 \mathrm{E}-01$ & $4.0073 E-01$ \\
\hline 1. $0 E+04$ & 0.75 & $6116 \mathrm{E}-01$ & $4.627027 \mathrm{E}-01$ & $7.3860 \mathrm{E}-01$ & $4.6233 E-01$ \\
\hline $1.0 E+04$ & 0.85 & $8.379263 E-01$ & $5.222048 \mathrm{E}-01$ & $8.3791 \mathrm{E}-01$ & $5.2124 \mathrm{E}-01$ \\
\hline 1. $0 E+04$ & 0.95 & $9.375577 \mathrm{E}-01$ & $5.687699 \mathrm{E}-01$ & $9.3754 \mathrm{E}-01$ & $5.6767 \mathrm{E}-01$ \\
\hline 1. $0 E+05$ & 5 & $2 E-02$ & 4.99421 & $E-02$ & $\mathrm{OE}-02$ \\
\hline 1. $0 E+05$ & 0.15 & $1.498497 \mathrm{E}-01$ & $1.498215 \mathrm{E}-01$ & $5 E-01$ & $1 E-01$ \\
\hline 1. $0 E+05$ & 0.25 & $2.497496 \mathrm{E}-01$ & $2.496801 \mathrm{E}-01$ & $2.4975 \mathrm{E}-01$ & $2.4966 \mathrm{E}-01$ \\
\hline $1.0 E+05$ & 0.35 & $3.496496 \mathrm{E}-01$ & $3.494796 \mathrm{E}-01$ & $3.4965 \mathrm{E}-01$ & $3.4944 \mathrm{E}-01$ \\
\hline 1. $0 \mathrm{E}+05$ & 0.45 & $4.495498 \mathrm{E}-01$ & $4.491170 \mathrm{E}-01$ & $4.4955 \mathrm{E}-01$ & $4.4902 E-01$ \\
\hline $1.0 \mathrm{E}+05$ & 0.55 & $5.494504 \mathrm{E}-01$ & $5.483125 \mathrm{E}-01$ & $5.4945 \mathrm{E}-01$ & $5.4811 \mathrm{E}-01$ \\
\hline 1. $0 \mathrm{E}+05$ & 0.65 & $6.493522 \mathrm{E}-01$ & $6.463054 \mathrm{E}-01$ & $6.4935 \mathrm{E}-01$ & $6.4588 \mathrm{E}-01$ \\
\hline $1.0 \mathrm{E}+05$ & 0.75 & $7.492574 \mathrm{E}-01$ & $7.410264 \mathrm{E}-01$ & $7.4926 \mathrm{E}-01$ & $7.4022 \mathrm{E}-01$ \\
\hline $1.0 \mathrm{E}+05$ & 0.85 & $8.491715 \mathrm{E}-01$ & $8.268474 \mathrm{E}-01$ & $8.4917 \mathrm{E}-01$ & $8.2555 \mathrm{E}-01$ \\
\hline 1. $0 \mathrm{E}+05$ & 0.95 & $9.491098 E-01$ & $8.884622 \mathrm{E}-01$ & $9.4911 \mathrm{E}-01$ & $8.8731 \mathrm{E}-01$ \\
\hline
\end{tabular}


Table A-2 (concluded):

$\begin{array}{llllll}1.0 \mathrm{E}+06 & 0.05 & 4.995005 \mathrm{E}-02 & 4.994534 \mathrm{E}-02 & 4.9950 \mathrm{E}-02 & 4.9943 \mathrm{E}-02 \\ 1.0 \mathrm{E}+06 & 0.15 & 1.498501 \mathrm{E}-01 & 1.498309 \mathrm{E}-01 & 1.4985 \mathrm{E}-01 & 1.4982 \mathrm{E}-01 \\ 1.0 \mathrm{E}+06 & 0.25 & 2.497503 \mathrm{E}-01 & 2.496956 \mathrm{E}-01 & 2.4975 \mathrm{E}-01 & 2.4968 \mathrm{E}-01 \\ 1.0 \mathrm{E}+06 & 0.35 & 3.496504 \mathrm{E}-01 & 3.495008 \mathrm{E}-01 & 3.4965 \mathrm{E}-01 & 3.4946 \mathrm{E}-01 \\ 1.0 \mathrm{E}+06 & 0.45 & 4.495508 \mathrm{E}-01 & 4.491435 \mathrm{E}-01 & 4.4955 \mathrm{E}-01 & 4.4905 \mathrm{E}-01 \\ 1.0 \mathrm{E}+06 & 0.55 & 5.494516 \mathrm{E}-01 & 5.483438 \mathrm{E}-01 & 5.4945 \mathrm{E}-01 & 5.4814 \mathrm{E}-01 \\ 1.0 \mathrm{E}+06 & 0.65 & 6.493536 \mathrm{E}-01 & 6.463407 \mathrm{E}-01 & 6.4935 \mathrm{E}-01 & 6.4592 \mathrm{E}-01 \\ 1.0 \mathrm{E}+06 & 0.75 & 7.492589 \mathrm{E}-01 & 7.410647 \mathrm{E}-01 & 7.4926 \mathrm{E}-01 & 7.4026 \mathrm{E}-01 \\ 1.0 \mathrm{E}+06 & 0.85 & 8.491731 \mathrm{E}-01 & 8.268879 \mathrm{E}-01 & 8.4917 \mathrm{E}-01 & 8.2560 \mathrm{E}-01 \\ 1.0 \mathrm{E}+06 & 0.95 & 9.491114 \mathrm{E}-01 & 8.885038 \mathrm{E}-01 & 9.4911 \mathrm{E}-01 & 8.8735 \mathrm{E}-01\end{array}$


RADIAL WELL FLOW PROBLEM IN A SATURATED, FRACTURED POROUS MEDIA BENCHMARK PROBLEMS FOR REPOSITORY SITING MODELS; NUREG/CR-3097; PP77-84 C. DAVID UPDEGRAFF

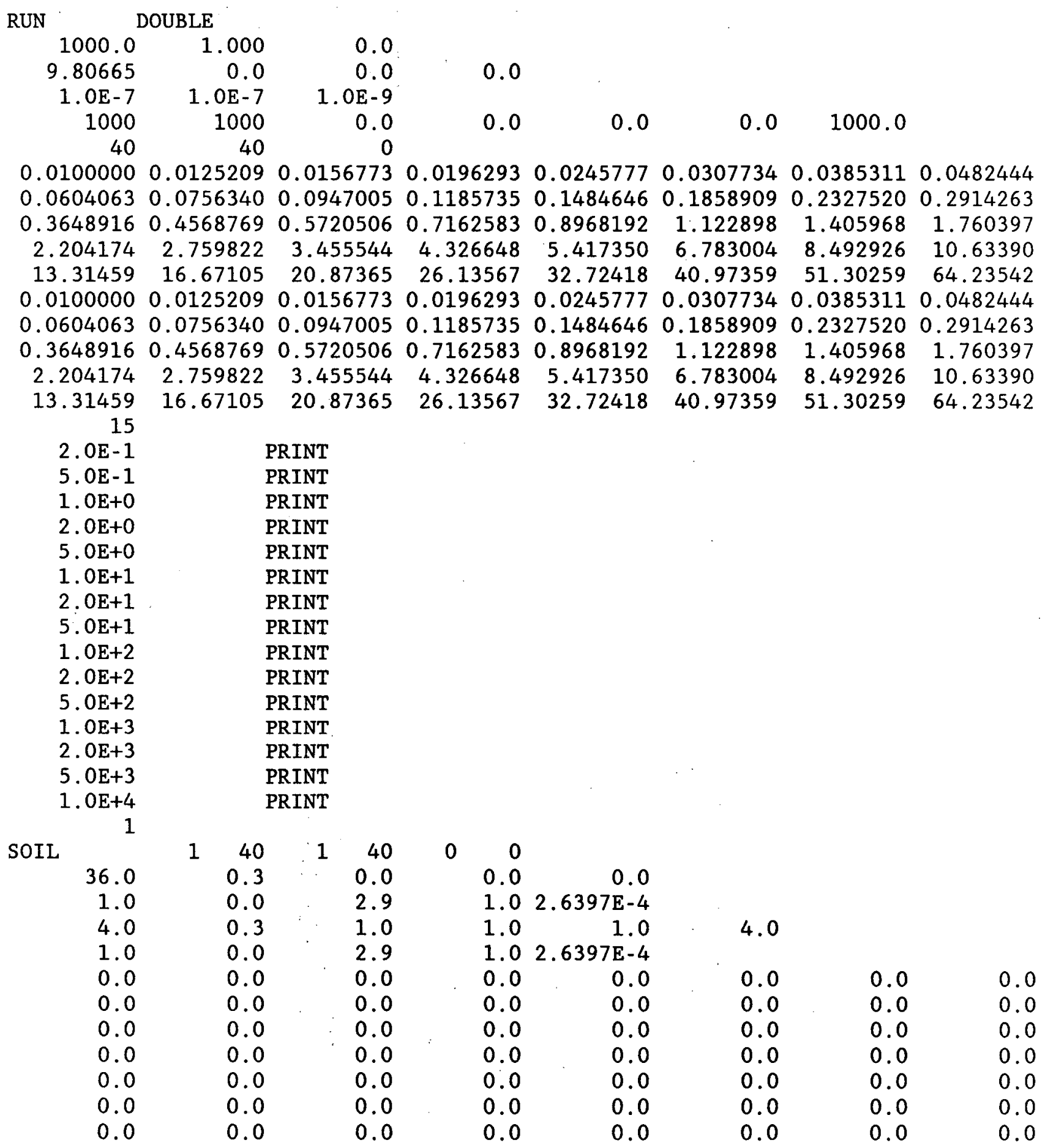




\begin{tabular}{|c|c|c|c|c|c|c|c|}
\hline 0.0 & 0.0 & 0.0 & 0.0 & 0.0 & 0.0 & 0.0 & 0.0 \\
\hline 0.0 & 0.0 & 0.0 & 0.0 & 0.0 & 0.0 & 0.0 & 0.0 \\
\hline 0.0 & 0.0 & 0.0 & 0.0 & 0.0 & 0.0 & 0.0 & 0.0 \\
\hline 0.0 & 0.0 & 0.0 & 0.0 & 0.0 & 0.0 & 0.0 & 0.0 \\
\hline 0.0 & 0.0 & 0.0 & 0.0 & 0.0 & 0.0 & 0.0 & 0.0 \\
\hline 0.0 & 0.0 & 0.0 & 0.0 & 0.0 & 0.0 & 0.0 & 0.0 \\
\hline 0.0 & 0.0 & 0.0 & 0.0 & 0.0 & 0.0 & 0.0 & 0.0 \\
\hline 0.0 & 0.0 & 0.0 & 0.0 & 0.0 & 0.0 & 0.0 & 0.0 \\
\hline 0.0 & 0.0 & 0.0 & 0.0 & 0.0 & 0.0 & 0.0 & 0.0 \\
\hline 0.0 & 0.0 & 0.0 & 0.0 & 0.0 & 0.0 & 0.0 & 0.0 \\
\hline 0.0 & 0.0 & 0.0 & 0.0 & 0.0 & 0.0 & 0.0 & 0.0 \\
\hline 0.0 & 0.0 & 0.0 & 0.0 & 0.0 & 0.0 & 0.0 & 0.0 \\
\hline 0.0 & 0.0 & 0.0 & 0.0 & 0.0 & 0.0 & 0.0 & 0.0 \\
\hline 0.0 & 0.0 & 0.0 & 0.0 & 0.0 & 0.0 & 0.0 & 0.0 \\
\hline 0.0 & 0.0 & 0.0 & 0.0 & 0.0 & 0.0 & 0.0 & 0.0 \\
\hline 0.0 & 0.0 & 0.0 & 0.0 & 0.0 & 0.0 & 0.0 & 0.0 \\
\hline 0.0 & 0.0 & 0.0 & 0.0 & 0.0 & 0.0 & 0.0 & 0.0 \\
\hline 0.0 & 0.0 & 0.0 & 0.0 & 0.0 & 0.0 & 0.0 & 0.0 \\
\hline 0.0 & 0.0 & 0.0 & 0.0 & 0.0 & 0.0 & 0.0 & 0.0 \\
\hline 0.0 & 0.0 & 0.0 & 0.0 & 0.0 & 0.0 & 0.0 & 0.0 \\
\hline 0.0 & 0.0 & 0.0 & 0.0 & 0.0 & 0.0 & 0.0 & 0.0 \\
\hline 0.0 & 0.0 & 0.0 & 0.0 & 0.0 & 0.0 & 0.0 & 0.0 \\
\hline 0.0 & 0.0 & 0.0 & 0.0 & 0.0 & 0.0 & 0.0 & 0.0 \\
\hline 0.0 & 0.0 & 0.0 & 0.0 & 0.0 & 0.0 & 0.0 & 0.0 \\
\hline 0.0 & 0.0 & 0.0 & 0.0 & 0.0 & 0.0 & 0.0 & 0.0 \\
\hline 0.0 & 0.0 & 0.0 & 0.0 & 0.0 & 0.0 & 0.0 & 0.0 \\
\hline 0.0 & 0.0 & 0.0 & 0.0 & 0.0 & 0.0 & 0.0 & 0.0 \\
\hline 0.0 & 0.0 & 0.0 & 0.0 & 0.0 & 0.0 & 0.0 & 0.0 \\
\hline 0.0 & 0.0 & 0.0 & 0.0 & 0.0 & 0.0 & 0.0 & 0.0 \\
\hline 0.0 & 0.0 & 0.0 & 0.0 & 0.0 & 0.0 & 0.0 & 0.0 \\
\hline 0.0 & 0.0 & 0.0 & 0.0 & 0.0 & 0.0 & 0.0 & 0.0 \\
\hline 0.0 & 0.0 & 0.0 & 0.0 & 0.0 & 0.0 & 0.0 & 0.0 \\
\hline 0.0 & 0.0 & 0.0 & 0.0 & 0.0 & 0.0 & 0.0 & 0.0 \\
\hline 0.0 & 0.0 & 0.0 & 0.0 & 0.0 & 0.0 & 0.0 & 0.0 \\
\hline 0.0 & 0.0 & 0.0 & 0.0 & 0.0 & 0.0 & 0.0 & 0.0 \\
\hline 0.0 & 0.0 & 0.0 & 0.0 & 0.0 & 0.0 & 0.0 & 0.0 \\
\hline 0.0 & 0.0 & 0.0 & 0.0 & 0.0 & 0.0 & 0.0 & 0.0 \\
\hline 0.0 & 0.0 & 0.0 & 0.0 & 0.0 & 0.0 & 0.0 & 0.0 \\
\hline 0.0 & 0.0 & 0.0 & 0.0 & 0.0 & 0.0 & 0.0 & 0.0 \\
\hline 0.0 & 0.0 & 0.0 & 0.0 & 0.0 & 0.0 & 0.0 & 0.0 \\
\hline 0.0 & 0.0 & 0.0 & 0.0 & 0.0 & 0.0 & 0.0 & 0.0 \\
\hline 0.0 & 0.0 & 0.0 & 0.0 & 0.0 & 0.0 & 0.0 & 0.0 \\
\hline 0.0 & 0.0 & 0.0 & 0.0 & 0.0 & 0.0 & 0.0 & 0.0 \\
\hline 0.0 & 0.0 & 0.0 & 0.0 & 0.0 & 0.0 & 0.0 & 0.0 \\
\hline 0.0 & 0.0 & 0.0 & 0.0 & 0.0 & 0.0 & 0.0 & 0.0 \\
\hline 0.0 & 0.0 & 0.0 & 0.0 & 0.0 & 0.0 & 0.0 & 0.0 \\
\hline 0.0 & 0.0 & 0.0 & 0.0 & 0.0 & 0.0 & 0.0 & 0.0 \\
\hline 0.0 & 0.0 & 0.0 & 0.0 & 0.0 & 0.0 & 0.0 & 0.0 \\
\hline 0.0 & 0.0 & 0.0 & 0.0 & 0.0 & 0.0 & 0.0 & 0.0 \\
\hline 0.0 & 0.0 & 0.0 & 0.0 & 0.0 & 0.0 & 0.0 & 0.0 \\
\hline 0.0 & 0.0 & 0.0 & 0.0 & 0.0 & 0.0 & 0.0 & 0.0 \\
\hline 0.0 & 0.0 & 0.0 & 0.0 & 0.0 & 0.0 & 0.0 & 0.0 \\
\hline 0.0 & 0.0 & 0.0 & 0.0 & 0.0 & 0.0 & 0.0 & 0.0 \\
\hline 0.0 & 0.0 & 0.0 & 0.0 & 0.0 & 0.0 & 0.0 & 0.0 \\
\hline
\end{tabular}




\begin{tabular}{|c|c|c|c|c|c|c|c|}
\hline 0.0 & 0.0 & 0.0 & 0.0 & 0.0 & 0.0 & 0.0 & 0.0 \\
\hline 0.0 & 0.0 & 0.0 & 0.0 & 0.0 & 0.0 & 0.0 & 0.0 \\
\hline 0.0 & 0.0 & 0.0 & 0.0 & 0.0 & 0.0 & 0.0 & 0.0 \\
\hline 0.0 & 0.0 & 0.0 & 0.0 & 0.0 & 0.0 & 0.0 & 0.0 \\
\hline 0.0 & 0.0 & 0.0 & 0.0 & 0.0 & 0.0 & 0.0 & 0.0 \\
\hline 0.0 & 0.0 & 0.0 & 0.0 & 0.0 & 0.0 & 0.0 & 0.0 \\
\hline 0.0 & 0.0 & 0.0 & 0.0 & 0.0 & 0.0 & 0.0 & 0.0 \\
\hline 0.0 & 0.0 & 0.0 & 0.0 & 0.0 & 0.0 & 0.0 & 0.0 \\
\hline 0.0 & 0.0 & 0.0 & 0.0 & 0.0 & 0.0 & 0.0 & 0.0 \\
\hline 0.0 & 0.0 & 0.0 & 0.0 & 0.0 & 0.0 & 0.0 & 0.0 \\
\hline 0.0 & 0.0 & 0.0 & 0.0 & 0.0 & 0.0 & 0.0 & 0.0 \\
\hline 0.0 & 0.0 & 0.0 & 0.0 & 0.0 & 0.0 & 0.0 & 0.0 \\
\hline 0.0 & 0.0 & 0.0 & 0.0 & 0.0 & 0.0 & 0.0 & 0.0 \\
\hline 0.0 & 0.0 & 0.0 & 0.0 & 0.0 & 0.0 & 0.0 & 0.0 \\
\hline 0.0 & 0.0 & 0.0 & 0.0 & 0.0 & 0.0 & 0.0 & 0.0 \\
\hline 0.0 & 0.0 & 0.0 & 0.0 & 0.0 & 0.0 & 0.0 & 0.0 \\
\hline 0.0 & 0.0 & 0.0 & 0.0 & 0.0 & 0.0 & 0.0 & 0.0 \\
\hline 0.0 & 0.0 & 0.0 & 0.0 & 0.0 & 0.0 & 0.0 & 0.0 \\
\hline 0.0 & 0.0 & 0.0 & 0.0 & 0.0 & 0.0 & 0.0 & 0.0 \\
\hline 0.0 & 0.0 & 0.0 & 0.0 & 0.0 & 0.0 & 0.0 & 0.0 \\
\hline 0.0 & 0.0 & 0.0 & 0.0 & 0.0 & 0.0 & 0.0 & 0.0 \\
\hline 0.0 & 0.0 & 0.0 & 0.0 & 0.0 & 0.0 & 0.0 & 0.0 \\
\hline 0.0 & 0.0 & 0.0 & 0.0 & 0.0 & 0.0 & 0.0 & 0.0 \\
\hline 0.0 & 0.0 & 0.0 & 0.0 & 0.0 & 0.0 & 0.0 & 0.0 \\
\hline 0.0 & 0.0 & 0.0 & 0.0 & 0.0 & 0.0 & 0.0 & 0.0 \\
\hline 0.0 & 0.0 & 0.0 & 0.0 & 0.0 & 0.0 & 0.0 & 0.0 \\
\hline 0.0 & 0.0 & 0.0 & 0.0 & 0.0 & 0.0 & 0.0 & 0.0 \\
\hline 0.0 & 0.0 & 0.0 & 0.0 & 0.0 & 0.0 & 0.0 & 0.0 \\
\hline 0.0 & 0.0 & 0.0 & 0.0 & 0.0 & 0.0 & 0.0 & 0.0 \\
\hline 0.0 & 0.0 & 0.0 & 0.0 & 0.0 & 0.0 & 0.0 & 0.0 \\
\hline 0.0 & 0.0 & 0.0 & 0.0 & 0.0 & 0.0 & 0.0 & 0.0 \\
\hline 0.0 & 0.0 & 0.0 & 0.0 & 0.0 & 0.0 & 0.0 & 0.0 \\
\hline 0.0 & 0.0 & 0.0 & 0.0 & 0.0 & 0.0 & 0.0 & 0.0 \\
\hline 0.0 & 0.0 & 0.0 & 0.0 & 0.0 & 0.0 & 0.0 & 0.0 \\
\hline 0.0 & 0.0 & 0.0 & 0.0 & 0.0 & 0.0 & 0.0 & 0.0 \\
\hline 0.0 & 0.0 & 0.0 & 0.0 & 0.0 & 0.0 & 0.0 & 0.0 \\
\hline 0.0 & 0.0 & 0.0 & 0.0 & 0.0 & 0.0 & 0.0 & 0.0 \\
\hline 0.0 & 0.0 & 0.0 & 0.0 & 0.0 & 0.0 & 0.0 & 0.0 \\
\hline 0.0 & 0.0 & 0.0 & 0.0 & 0.0 & 0.0 & 0.0 & 0.0 \\
\hline 0.0 & 0.0 & 0.0 & 0.0 & 0.0 & 0.0 & 0.0 & 0.0 \\
\hline 0.0 & 0.0 & 0.0 & 0.0 & 0.0 & 0.0 & 0.0 & 0.0 \\
\hline 0.0 & 0.0 & 0.0 & 0.0 & 0.0 & 0.0 & 0.0 & 0.0 \\
\hline 0.0 & 0.0 & 0.0 & 0.0 & 0.0 & 0.0 & 0.0 & 0.0 \\
\hline 0.0 & 0.0 & 0.0 & 0.0 & 0.0 & 0.0 & 0.0 & 0.0 \\
\hline 0.0 & 0.0 & 0.0 & 0.0 & 0.0 & 0.0 & 0.0 & 0.0 \\
\hline 0.0 & 0.0 & 0.0 & 0.0 & 0.0 & 0.0 & 0.0 & 0.0 \\
\hline 0.0 & 0.0 & 0.0 & 0.0 & 0.0 & 0.0 & 0.0 & 0.0 \\
\hline 0.0 & 0.0 & 0.0 & 0.0 & 0.0 & 0.0 & 0.0 & 0.0 \\
\hline 0.0 & 0.0 & 0.0 & 0.0 & 0.0 & 0.0 & 0.0 & 0.0 \\
\hline 0.0 & 0.0 & 0.0 & 0.0 & 0.0 & 0.0 & 0.0 & 0.0 \\
\hline 0.0 & 0.0 & 0.0 & 0.0 & 0.0 & 0.0 & 0.0 & 0.0 \\
\hline 0.0 & 0.0 & 0.0 & 0.0 & 0.0 & 0.0 & 0.0 & 0.0 \\
\hline 0.0 & 0.0 & 0.0 & 0.0 & 0.0 & 0.0 & 0.0 & 0.0 \\
\hline 0.0 & 0.0 & 0.0 & 0.0 & 0.0 & 0.0 & 0.0 & 0.0 \\
\hline
\end{tabular}




\begin{tabular}{|c|c|c|c|c|c|c|c|}
\hline 0.0 & 0.0 & 0.0 & 0.0 & 0.0 & 0.0 & 0.0 & 0.0 \\
\hline 0.0 & 0.0 & 0.0 & 0.0 & 0.0 & 0.0 & 0.0 & 0.0 \\
\hline 0.0 & 0.0 & 0.0 & 0.0 & 0.0 & 0.0 & 0.0 & 0.0 \\
\hline 0.0 & 0.0 & 0.0 & 0.0 & 0.0 & 0.0 & 0.0 & 0.0 \\
\hline 0.0 & 0.0 & 0.0 & 0.0 & 0.0 & 0.0 & 0.0 & 0.0 \\
\hline 0.0 & 0.0 & 0.0 & 0.0 & 0.0 & 0.0 & 0.0 & 0.0 \\
\hline 0.0 & 0.0 & 0.0 & 0.0 & 0.0 & 0.0 & 0.0 & 0.0 \\
\hline 0.0 & 0.0 & 0.0 & 0.0 & 0.0 & 0.0 & 0.0 & 0.0 \\
\hline 0.0 & 0.0 & 0.0 & 0.0 & 0.0 & 0.0 & 0.0 & 0.0 \\
\hline 0.0 & 0.0 & 0.0 & 0.0 & 0.0 & 0.0 & 0.0 & 0.0 \\
\hline 0.0 & 0.0 & 0.0 & 0.0 & 0.0 & 0.0 & 0.0 & 0.0 \\
\hline 0.0 & 0.0 & 0.0 & 0.0 & 0.0 & 0.0 & 0.0 & 0.0 \\
\hline 0.0 & 0.0 & 0.0 & 0.0 & 0.0 & 0.0 & 0.0 & 0.0 \\
\hline 0.0 & 0.0 & 0.0 & 0.0 & 0.0 & 0.0 & 0.0 & 0.0 \\
\hline 0.0 & 0.0 & 0.0 & 0.0 & 0.0 & 0.0 & 0.0 & 0.0 \\
\hline 0.0 & 0.0 & 0.0 & 0.0 & 0.0 & 0.0 & 0.0 & 0.0 \\
\hline 0.0 & 0.0 & 0.0 & 0.0 & 0.0 & 0.0 & 0.0 & 0.0 \\
\hline 0.0 & 0.0 & 0.0 & 0.0 & 0.0 & 0.0 & 0.0 & 0.0 \\
\hline 0.0 & 0.0 & 0.0 & 0.0 & 0.0 & 0.0 & 0.0 & 0.0 \\
\hline 0.0 & 0.0 & 0.0 & 0.0 & 0.0 & 0.0 & 0.0 & 0.0 \\
\hline 0.0 & 0.0 & 0.0 & 0.0 & 0.0 & 0.0 & 0.0 & 0.0 \\
\hline 0.0 & 0.0 & 0.0 & 0.0 & 0.0 & 0.0 & 0.0 & 0.0 \\
\hline 0.0 & 0.0 & 0.0 & 0.0 & 0.0 & 0.0 & 0.0 & 0.0 \\
\hline 0.0 & 0.0 & 0.0 & 0.0 & 0.0 & 0.0 & 0.0 & 0.0 \\
\hline 0.0 & 0.0 & 0.0 & 0.0 & 0.0 & 0.0 & 0.0 & 0.0 \\
\hline 0.0 & 0.0 & 0.0 & 0.0 & 0.0 & 0.0 & 0.0 & 0.0 \\
\hline 0.0 & 0.0 & 0.0 & 0.0 & 0.0 & 0.0 & 0.0 & 0.0 \\
\hline 0.0 & 0.0 & 0.0 & 0.0 & 0.0 & 0.0 & 0.0 & 0.0 \\
\hline 0.0 & 0.0 & 0.0 & 0.0 & 0.0 & 0.0 & 0.0 & 0.0 \\
\hline 0.0 & 0.0 & 0.0 & 0.0 & 0.0 & 0.0 & 0.0 & 0.0 \\
\hline 0.0 & 0.0 & 0.0 & 0.0 & 0.0 & 0.0 & 0.0 & 0.0 \\
\hline 0.0 & 0.0 & 0.0 & 0.0 & 0.0 & 0.0 & 0.0 & 0.0 \\
\hline 0.0 & 0.0 & 0.0 & 0.0 & 0.0 & 0.0 & 0.0 & 0.0 \\
\hline 0.0 & 0.0 & 0.0 & 0.0 & 0.0 & 0.0 & 0.0 & 0.0 \\
\hline 0.0 & 0.0 & 0.0 & 0.0 & 0.0 & 0.0 & 0.0 & 0.0 \\
\hline 0.0 & 0.0 & 0.0 & 0.0 & 0.0 & 0.0 & 0.0 & 0.0 \\
\hline 0.0 & 0.0 & 0.0 & 0.0 & 0.0 & 0.0 & 0.0 & 0.0 \\
\hline 0.0 & 0.0 & 0.0 & 0.0 & 0.0 & 0.0 & 0.0 & 0.0 \\
\hline 0.0 & 0.0 & 0.0 & 0.0 & 0.0 & 0.0 & 0.0 & 0.0 \\
\hline 0.0 & 0.0 & 0.0 & 0.0 & 0.0 & 0.0 & 0.0 & 0.0 \\
\hline 0.0 & 0.0 & 0.0 & 0.0 & 0.0 & 0.0 & 0.0 & 0.0 \\
\hline 0.0 & 0.0 & 0.0 & 0.0 & 0.0 & 0.0 & 0.0 & 0.0 \\
\hline 0.0 & 0.0 & 0.0 & 0.0 & 0.0 & 0.0 & 0.0 & 0.0 \\
\hline 0.0 & 0.0 & 0.0 & 0.0 & 0.0 & 0.0 & 0.0 & 0.0 \\
\hline 0.0 & 0.0 & 0.0 & 0.0 & 0.0 & 0.0 & 0.0 & 0.0 \\
\hline 0.0 & 0.0 & 0.0 & 0.0 & 0.0 & 0.0 & 0.0 & 0.0 \\
\hline 0.0 & 0.0 & 0.0 & 0.0 & 0.0 & 0.0 & 0.0 & 0.0 \\
\hline 0.0 & 0.0 & 0.0 & 0.0 & 0.0 & 0.0 & 0.0 & 0.0 \\
\hline 0.0 & 0.0 & 0.0 & 0.0 & 0.0 & 0.0 & 0.0 & 0.0 \\
\hline 0.0 & 0.0 & 0.0 & 0.0 & 0.0 & 0.0 & 0.0 & 0.0 \\
\hline 0.0 & 0.0 & 0.0 & 0.0 & 0.0 & 0.0 & 0.0 & 0.0 \\
\hline 0.0 & 0.0 & 0.0 & 0.0 & 0.0 & 0.0 & 0.0 & 0.0 \\
\hline 0.0 & 0.0 & 0.0 & 0.0 & 0.0 & 0.0 & 0.0 & 0.0 \\
\hline 0.0 & 0.0 & 0.0 & 0.0 & 0.0 & 0.0 & 0.0 & 0.0 \\
\hline
\end{tabular}




\begin{tabular}{|c|c|c|c|c|c|c|c|}
\hline 0.0 & 0.0 & 0.0 & 0.0 & 0.0 & 0.0 & 0.0 & 0.0 \\
\hline 0.0 & 0.0 & 0.0 & 0.0 & 0.0 & 0.0 & 0.0 & 0.0 \\
\hline 0.0 & 0.0 & 0.0 & 0.0 & 0.0 & 0.0 & 0.0 & 0.0 \\
\hline 0.0 & 0.0 & 0.0 & 0.0 & 0.0 & 0.0 & 0.0 & 0.0 \\
\hline 0.0 & 0.0 & 0.0 & 0.0 & 0.0 & 0.0 & 0.0 & 0.0 \\
\hline 0.0 & 0.0 & 0.0 & 0.0 & 0.0 & 0.0 & 0.0 & 0.0 \\
\hline 0.0 & 0.0 & 0.0 & 0.0 & 0.0 & 0.0 & 0.0 & 0.0 \\
\hline 0.0 & 0.0 & 0.0 & 0.0 & 0.0 & 0.0 & 0.0 & 0.0 \\
\hline 0.0 & 0.0 & 0.0 & 0.0 & 0.0 & 0.0 & 0.0 & 0.0 \\
\hline 0.0 & 0.0 & 0.0 & 0.0 & 0.0 & 0.0 & 0.0 & 0.0 \\
\hline 0.0 & 0.0 & 0.0 & 0.0 & 0.0 & 0.0 & 0.0 & 0.0 \\
\hline 0.0 & 0.0 & 0.0 & 0.0 & 0.0 & 0.0 & 0.0 & 0.0 \\
\hline 0.0 & 0.0 & 0.0 & 0.0 & 0.0 & 0.0 & 0.0 & 0.0 \\
\hline 0.0 & 0.0 & 0.0 & 0.0 & 0.0 & 0.0 & 0.0 & 0.0 \\
\hline 0.0 & 0.0 & 0.0 & 0.0 & 0.0 & 0.0 & 0.0 & 0.0 \\
\hline 0.0 & 0.0 & 0.0 & 0.0 & 0.0 & 0.0 & 0.0 & 0.0 \\
\hline 0.0 & 0.0 & 0.0 & 0.0 & 0.0 & 0.0 & 0.0 & 0.0 \\
\hline 0.0 & 0.0 & 0.0 & 0.0 & 0.0 & 0.0 & 0.0 & 0.0 \\
\hline 0.0 & 0.0 & 0.0 & 0.0 & 0.0 & 0.0 & 0.0 & 0.0 \\
\hline 0.0 & 0.0 & 0.0 & 0.0 & 0.0 & 0.0 & 0.0 & 0.0 \\
\hline 0.0 & 0.0 & 0.0 & 0.0 & 0.0 & 0.0 & 0.0 & 0.0 \\
\hline 0.0 & 0.0 & 0.0 & 0.0 & 0.0 & 0.0 & 0.0 & 0.0 \\
\hline 0.0 & 0.0 & 0.0 & 0.0 & 0.0 & 0.0 & 0.0 & 0.0 \\
\hline 0.0 & 0.0 & 0.0 & 0.0 & 0.0 & 0.0 & 0.0 & 0.0 \\
\hline 0.0 & 0.0 & 0.0 & 0.0 & 0.0 & 0.0 & 0.0 & 0.0 \\
\hline 0.0 & 0.0 & 0.0 & 0.0 & 0.0 & 0.0 & 0.0 & 0.0 \\
\hline 0.0 & 0.0 & 0.0 & 0.0 & 0.0 & 0.0 & 0.0 & 0.0 \\
\hline 0.0 & 0.0 & 0.0 & 0.0 & 0.0 & 0.0 & 0.0 & 0.0 \\
\hline 0.0 & 0.0 & 0.0 & 0.0 & 0.0 & 0.0 & 0.0 & 0.0 \\
\hline 0.0 & 0.0 & 0.0 & 0.0 & 0.0 & 0.0 & 0.0 & 0.0 \\
\hline 0.0 & 0.0 & 0.0 & 0.0 & 0.0 & 0.0 & 0.0 & 0.0 \\
\hline 0.0 & 0.0 & 0.0 & 0.0 & 0.0 & 0.0 & 0.0 & 0.0 \\
\hline 0.0 & 0.0 & 0.0 & 0.0 & 0.0 & 0.0 & 0.0 & 0.0 \\
\hline 0.0 & 0.0 & 0.0 & 0.0 & 0.0 & 0.0 & 0.0 & 0.0 \\
\hline 0.0 & 0.0 & 0.0 & 0.0 & 0.0 & 0.0 & 0.0 & 0.0 \\
\hline 0.0 & 0.0 & 0.0 & 0.0 & 0.0 & 0.0 & 0.0 & 0.0 \\
\hline 0.0 & 0.0 & 0.0 & 0.0 & 0.0 & 0.0 & 0.0 & 0.0 \\
\hline 0.0 & 0.0 & 0.0 & 0.0 & 0.0 & 0.0 & 0.0 & 0.0 \\
\hline 0.0 & 0.0 & 0.0 & 0.0 & 0.0 & 0.0 & 0.0 & 0.0 \\
\hline 0.0 & 0.0 & 0.0 & 0.0 & 0.0 & 0.0 & 0.0 & 0.0 \\
\hline 0.0 & 0.0 & 0.0 & 0.0 & 0.0 & 0.0 & 0.0 & 0.0 \\
\hline 0.0 & 0.0 & 0.0 & 0.0 & 0.0 & 0.0 & 0.0 & 0.0 \\
\hline 0.0 & 0.0 & 0.0 & 0.0 & 0.0 & 0.0 & 0.0 & 0.0 \\
\hline 0.0 & 0.0 & 0.0 & 0.0 & 0.0 & 0.0 & 0.0 & 0.0 \\
\hline 0.0 & 0.0 & 0.0 & 0.0 & 0.0 & 0.0 & 0.0 & 0.0 \\
\hline 0.0 & 0.0 & 0.0 & 0.0 & 0.0 & 0.0 & 0.0 & 0.0 \\
\hline 0.0 & 0.0 & 0.0 & 0.0 & 0.0 & 0.0 & 0.0 & 0.0 \\
\hline 0.0 & 0.0 & 0.0 & 0.0 & 0.0 & 0.0 & 0.0 & 0.0 \\
\hline 0.0 & 0.0 & 0.0 & 0.0 & 0.0 & 0.0 & 0.0 & 0.0 \\
\hline 0.0 & 0.0 & 0.0 & 0.0 & 0.0 & 0.0 & 0.0 & 0.0 \\
\hline 0.0 & 0.0 & 0.0 & 0.0 & 0.0 & 0.0 & 0.0 & 0.0 \\
\hline 0.0 & 0.0 & 0.0 & 0.0 & 0.0 & 0.0 & 0.0 & 0.0 \\
\hline 0.0 & 0.0 & 0.0 & 0.0 & 0.0 & 0.0 & 0.0 & 0.0 \\
\hline 0.0 & 0.0 & 0.0 & 0.0 & 0.0 & 0.0 & 0.0 & 0.0 \\
\hline
\end{tabular}




\begin{tabular}{|c|c|c|c|c|c|c|c|}
\hline 0.0 & 0.0 & 0.0 & 0.0 & 0.0 & 0.0 & 0.0 & 0.0 \\
\hline 0.0 & 0.0 & 0.0 & 0.0 & 0.0 & 0.0 & 0.0 & 0.0 \\
\hline 0.0 & 0.0 & 0.0 & 0.0 & 0.0 & 0.0 & 0.0 & 0.0 \\
\hline 0.0 & 0.0 & 0.0 & 0.0 & 0.0 & 0.0 & 0.0 & 0.0 \\
\hline 0.0 & 0.0 & 0.0 & 0.0 & 0.0 & 0.0 & 0.0 & 0.0 \\
\hline 0.0 & 0.0 & 0.0 & 0.0 & 0.0 & 0.0 & 0.0 & 0.0 \\
\hline 0.0 & 0.0 & 0.0 & 0.0 & 0.0 & 0.0 & 0.0 & 0.0 \\
\hline 0.0 & 0.0 & 0.0 & 0.0 & 0.0 & 0.0 & 0.0 & 0.0 \\
\hline 0.0 & 0.0 & 0.0 & 0.0 & 0.0 & 0.0 & 0.0 & 0.0 \\
\hline 0.0 & 0.0 & 0.0 & 0.0 & 0.0 & 0.0 & 0.0 & 0.0 \\
\hline 0.0 & 0.0 & 0.0 & 0.0 & 0.0 & 0.0 & 0.0 & 0.0 \\
\hline 0.0 & 0.0 & 0.0 & 0.0 & 0.0 & 0.0 & 0.0 & 0.0 \\
\hline 0.0 & 0.0 & 0.0 & 0.0 & 0.0 & 0.0 & 0.0 & 0.0 \\
\hline 0.0 & 0.0 & 0.0 & 0.0 & 0.0 & 0.0 & 0.0 & 0.0 \\
\hline 0.0 & 0.0 & 0.0 & 0.0 & 0.0 & 0.0 & 0.0 & 0.0 \\
\hline 0.0 & 0.0 & 0.0 & 0.0 & 0.0 & 0.0 & 0.0 & 0.0 \\
\hline 0.0 & 0.0 & 0.0 & 0.0 & 0.0 & 0.0 & 0.0 & 0.0 \\
\hline 0.0 & 0.0 & 0.0 & 0.0 & 0.0 & 0.0 & 0.0 & 0.0 \\
\hline 0.0 & 0.0 & 0.0 & 0.0 & 0.0 & 0.0 & 0.0 & 0.0 \\
\hline 0.0 & 0.0 & 0.0 & 0.0 & 0.0 & 0.0 & 0.0 & 0.0 \\
\hline 0.0 & 0.0 & 0.0 & 0.0 & 0.0 & 0.0 & 0.0 & 0.0 \\
\hline 0.0 & 0.0 & 0.0 & 0.0 & 0.0 & 0.0 & 0.0 & 0.0 \\
\hline 0.0 & 0.0 & 0.0 & 0.0 & 0.0 & 0.0 & 0.0 & 0.0 \\
\hline 0.0 & 0.0 & 0.0 & 0.0 & 0.0 & 0.0 & 0.0 & 0.0 \\
\hline 0.0 & 0.0 & 0.0 & 0.0 & 0.0 & 0.0 & 0.0 & 0.0 \\
\hline 0.0 & 0.0 & 0.0 & 0.0 & 0.0 & 0.0 & 0.0 & 0.0 \\
\hline 0.0 & 0.0 & 0.0 & 0.0 & 0.0 & 0.0 & 0.0 & 0.0 \\
\hline 0.0 & 0.0 & 0.0 & 0.0 & 0.0 & 0.0 & 0.0 & 0.0 \\
\hline 0.0 & 0.0 & 0.0 & 0.0 & 0.0 & 0.0 & 0.0 & 0.0 \\
\hline 0.0 & 0.0 & 0.0 & 0.0 & 0.0 & 0.0 & 0.0 & 0.0 \\
\hline 0.0 & 0.0 & 0.0 & 0.0 & 0.0 & 0.0 & 0.0 & 0.0 \\
\hline 0.0 & 0.0 & 0.0 & 0.0 & 0.0 & 0.0 & 0.0 & 0.0 \\
\hline 0.0 & 0.0 & 0.0 & 0.0 & 0.0 & 0.0 & 0.0 & 0.0 \\
\hline 0.0 & 0.0 & 0.0 & 0.0 & 0.0 & 0.0 & 0.0 & 0.0 \\
\hline 0.0 & 0.0 & 0.0 & 0.0 & 0.0 & 0.0 & 0.0 & 0.0 \\
\hline 0.0 & 0.0 & 0.0 & 0.0 & 0.0 & 0.0 & 0.0 & 0.0 \\
\hline 0.0 & 0.0 & 0.0 & 0.0 & 0.0 & 0.0 & 0.0 & 0.0 \\
\hline 0.0 & 0.0 & 0.0 & 0.0 & 0.0 & 0.0 & 0.0 & 0.0 \\
\hline 0.0 & 0.0 & 0.0 & 0.0 & 0.0 & 0.0 & 0.0 & 0.0 \\
\hline 0.0 & 0.0 & 0.0 & 0.0 & 0.0 & 0.0 & 0.0 & 0.0 \\
\hline 0.0 & 0.0 & 0.0 & 0.0 & 0.0 & 0.0 & 0.0 & 0.0 \\
\hline 0.0 & 0.0 & 0.0 & 0.0 & 0.0 & 0.0 & 0.0 & 0.0 \\
\hline 0.0 & 0.0 & 0.0 & 0.0 & 0.0 & 0.0 & 0.0 & 0.0 \\
\hline 0.0 & 0.0 & 0.0 & 0.0 & 0.0 & 0.0 & 0.0 & 0.0 \\
\hline 0.0 & 0.0 & 0.0 & 0.0 & 0.0 & 0.0 & 0.0 & 0.0 \\
\hline 0.0 & 0.0 & 0.0 & 0.0 & 0.0 & 0.0 & 0.0 & 0.0 \\
\hline 0.0 & 0.0 & 0.0 & 0.0 & 0.0 & 0.0 & 0.0 & 0.0 \\
\hline 0.0 & 0.0 & 0.0 & 0.0 & 0.0 & 0.0 & 0.0 & 0.0 \\
\hline 0.0 & 0.0 & 0.0 & 0.0 & 0.0 & 0.0 & 0.0 & 0.0 \\
\hline 0.0 & 0.0 & 0.0 & 0.0 & 0.0 & 0.0 & 0.0 & 0.0 \\
\hline 0.0 & 0.0 & 0.0 & 0.0 & 0.0 & 0.0 & 0.0 & 0.0 \\
\hline 0.0 & 0.0 & 0.0 & 0.0 & 0.0 & 0.0 & 0.0 & 0.0 \\
\hline 0.0 & 0.0 & 0.0 & 0.0 & 0.0 & 0.0 & 0.0 & 0.0 \\
\hline 0.0 & 0.0 & 0.0 & 0.0 & 0.0 & 0.0 & 0.0 & 0.0 \\
\hline
\end{tabular}




\begin{tabular}{|c|c|c|c|c|c|c|c|}
\hline 0.0 & 0.0 & 0.0 & 0.0 & 0.0 & 0.0 & 0.0 & 0.0 \\
\hline 0.0 & 0.0 & 0.0 & 0.0 & 0.0 & 0.0 & 0.0 & 0.0 \\
\hline 0.0 & 0.0 & 0.0 & 0.0 & 0.0 & 0.0 & 0.0 & 0.0 \\
\hline 0.0 & 0.0 & 0.0 & 0.0 & 0.0 & 0.0 & 0.0 & 0.0 \\
\hline 0.0 & 0.0 & 0.0 & 0.0 & 0.0 & 0.0 & 0.0 & 0.0 \\
\hline 0.0 & 0.0 & 0.0 & 0.0 & 0.0 & 0.0 & 0.0 & 0.0 \\
\hline 0.0 & 0.0 & 0.0 & 0.0 & 0.0 & 0.0 & 0.0 & 0.0 \\
\hline 0.0 & 0.0 & 0.0 & 0.0 & 0.0 & 0.0 & 0.0 & 0.0 \\
\hline 0.0 & 0.0 & 0.0 & 0.0 & 0.0 & 0.0 & 0.0 & 0.0 \\
\hline 0.0 & 0.0 & 0.0 & 0.0 & 0.0 & 0.0 & 0.0 & 0.0 \\
\hline 0.0 & 0.0 & 0.0 & 0.0 & 0.0 & 0.0 & 0.0 & 0.0 \\
\hline 0.0 & 0.0 & 0.0 & 0.0 & 0.0 & 0.0 & 0.0 & 0.0 \\
\hline 0.0 & 0.0 & 0.0 & 0.0 & 0.0 & 0.0 & 0.0 & 0.0 \\
\hline 0.0 & 0.0 & 0.0 & 0.0 & 0.0 & 0.0 & 0.0 & 0.0 \\
\hline 0.0 & 0.0 & 0.0 & 0.0 & 0.0 & 0.0 & 0.0 & 0.0 \\
\hline 0.0 & 0.0 & 0.0 & 0.0 & 0.0 & 0.0 & 0.0 & 0.0 \\
\hline 0.0 & 0.0 & 0.0 & 0.0 & 0.0 & 0.0 & 0.0 & 0.0 \\
\hline 0.0 & 0.0 & 0.0 & 0.0 & 0.0 & 0.0 & 0.0 & 0.0 \\
\hline 0.0 & 0.0 & 0.0 & 0.0 & 0.0 & 0.0 & 0.0 & 0.0 \\
\hline 0.0 & 0.0 & 0.0 & 0.0 & 0.0 & 0.0 & 0.0 & 0.0 \\
\hline 0.0 & 0.0 & 0.0 & 0.0 & 0.0 & 0.0 & 0.0 & 0.0 \\
\hline 0.0 & 0.0 & 0.0 & 0.0 & 0.0 & 0.0 & 0.0 & 0.0 \\
\hline 0.0 & 0.0 & 0.0 & 0.0 & 0.0 & 0.0 & 0.0 & 0.0 \\
\hline 0.0 & 0.0 & 0.0 & 0.0 & 0.0 & 0.0 & 0.0 & 0.0 \\
\hline 0.0 & 0.0 & 0.0 & 0.0 & 0.0 & 0.0 & 0.0 & 0.0 \\
\hline 0.0 & 0.0 & 0.0 & 0.0 & 0.0 & 0.0 & 0.0 & 0.0 \\
\hline 0.0 & 0.0 & 0.0 & 0.0 & 0.0 & 0.0 & 0.0 & 0.0 \\
\hline 0.0 & 0.0 & 0.0 & 0.0 & 0.0 & 0.0 & 0.0 & 0.0 \\
\hline 0.0 & 0.0 & 0.0 & 0.0 & 0.0 & 0.0 & 0.0 & 0.0 \\
\hline 0.0 & 0.0 & 0.0 & 0.0 & 0.0 & 0.0 & 0.0 & 0.0 \\
\hline 0.0 & 0.0 & 0.0 & 0.0 & 0.0 & 0.0 & 0.0 & 0.0 \\
\hline 0.0 & 0.0 & 0.0 & 0.0 & 0.0 & 0.0 & 0.0 & 0.0 \\
\hline 0.0 & 0.0 & $0: 0$ & 0.0 & 0.0 & 0.0 & 0.0 & 0.0 \\
\hline 0.0 & 0.0 & 0.0 & 0.0 & 0.0 & 0.0 & 0.0 & 0.0 \\
\hline 0.0 & 0.0 & 0.0 & 0.0 & 0.0 & 0.0 & 0.0 & 0.0 \\
\hline 0.0 & 0.0 & 0.0 & 0.0 & 0.0 & 0.0 & 0.0 & 0.0 \\
\hline 0.0 & 0.0 & 0.0 & 0.0 & 0.0 & 0.0 & 0.0 & 0.0 \\
\hline 0.0 & 0.0 & 0.0 & 0.0 & 0.0 & 0.0 & 0.0 & 0.0 \\
\hline 0.0 & 0.0 & 0.0 & 0.0 & 0.0 & 0.0 & 0.0 & 0.0 \\
\hline 0.0 & 0.0 & 0.0 & 0.0 & 0.0 & 0.0 & 0.0 & 0.0 \\
\hline 0.0 & 0.0 & 0.0 & 0.0 & 0.0 & 0.0 & 0.0 & 0.0 \\
\hline 0.0 & 0.0 & 0.0 & 0.0 & 0.0 & 0.0 & 0.0 & 0.0 \\
\hline 0.0 & 0.0 & 0.0 & 0.0 & 0.0 & 0.0 & 0.0 & 0.0 \\
\hline 0.0 & 0.0 & 0.0 & 0.0 & 0.0 & 0.0 & 0.0 & 0.0 \\
\hline 0.0 & 0.0 & 0.0 & 0.0 & 0.0 & 0.0 & 0.0 & 0.0 \\
\hline 0.0 & 0.0 & 0.0 & 0.0 & 0.0 & 0.0 & 0.0 & 0.0 \\
\hline 0.0 & 0.0 & 0.0 & 0.0 & 0.0 & $0: 0$ & 0.0 & 0.0 \\
\hline 0.0 & 0.0 & 0.0 & 0.0 & 0.0 & 0.0 & 0.0 & 0.0 \\
\hline 0.0 & 0.0 & 0.0 & 0.0 & 0.0 & 0.0 & 0.0 & 0.0 \\
\hline 0.0 & 0.0 & 0.0 & 0.0 & 0.0 & 0.0 & 0.0 & 0.0 \\
\hline 0.0 & 0.0 & 0.0 & 0.0 & 0.0 & 0.0 & 0.0 & 0.0 \\
\hline 0.0 & 0.0 & 0.0 & 0.0 & 0.0 & 0.0 & 0.0 & 0.0 \\
\hline 0.0 & 0.0 & 0.0 & 0.0 & 0.0 & 0.0 & 0.0 & 0.0 \\
\hline 0.0 & 0.0 & 0.0 & 0.0 & 0.0 & 0.0 & 0.0 & 0.0 \\
\hline
\end{tabular}




\begin{tabular}{|c|c|c|c|c|c|c|c|}
\hline 0.0 & 0.0 & 0.0 & 0.0 & 0.0 & 0.0 & 0.0 & 0.0 \\
\hline 0.0 & 0.0 & 0.0 & 0.0 & 0.0 & 0.0 & 0.0 & 0.0 \\
\hline 0.0 & 0.0 & 0.0 & 0.0 & 0.0 & 0.0 & 0.0 & 0.0 \\
\hline 0.0 & 0.0 & 0.0 & 0.0 & 0.0 & 0.0 & 0.0 & 0.0 \\
\hline 0.0 & 0.0 & 0.0 & 0.0 & 0.0 & 0.0 & 0.0 & 0.0 \\
\hline 0.0 & 0.0 & 0.0 & 0.0 & 0.0 & 0.0 & 0.0 & 0.0 \\
\hline 0.0 & 0.0 & 0.0 & 0.0 & 0.0 & 0.0 & 0.0 & 0.0 \\
\hline 0.0 & 0.0 & 0.0 & 0.0 & 0.0 & 0.0 & 0.0 & 0.0 \\
\hline 0.0 & 0.0 & 0.0 & 0.0 & 0.0 & 0.0 & 0.0 & 0.0 \\
\hline 0.0 & 0.0 & 0.0 & 0.0 & 0.0 & 0.0 & 0.0 & 0.0 \\
\hline 0.0 & 0.0 & 0.0 & 0.0 & 0.0 & 0.0 & 0.0 & 0.0 \\
\hline 0.0 & 0.0 & 0.0 & 0.0 & 0.0 & 0.0 & 0.0 & 0.0 \\
\hline 0.0 & 0.0 & 0.0 & 0.0 & 0.0 & 0.0 & 0.0 & 0.0 \\
\hline 0.0 & 0.0 & 0.0 & 0.0 & 0.0 & 0.0 & 0.0 & 0.0 \\
\hline 0.0 & 0.0 & 0.0 & 0.0 & 0.0 & 0.0 & 0.0 & 0.0 \\
\hline 0.0 & 0.0 & 0.0 & 0.0 & 0.0 & 0.0 & 0.0 & 0.0 \\
\hline 0.0 & 0.0 & 0.0 & 0.0 & 0.0 & 0.0 & 0.0 & 0.0 \\
\hline 0.0 & 0.0 & 0.0 & 0.0 & 0.0 & 0.0 & 0.0 & 0.0 \\
\hline 0.0 & 0.0 & 0.0 & 0.0 & 0.0 & 0.0 & 0.0 & 0.0 \\
\hline 0.0 & 0.0 & 0.0 & 0.0 & 0.0 & 0.0 & 0.0 & 0.0 \\
\hline 0.0 & 0.0 & 0.0 & 0.0 & 0.0 & 0.0 & 0.0 & 0.0 \\
\hline 0.0 & 0.0 & 0.0 & 0.0 & 0.0 & 0.0 & 0.0 & 0.0 \\
\hline 0.0 & 0.0 & 0.0 & 0.0 & 0.0 & 0.0 & 0.0 & 0.0 \\
\hline 0.0 & 0.0 & 0.0 & 0.0 & 0.0 & 0.0 & 0.0 & 0.0 \\
\hline 0.0 & 0.0 & 0.0 & 0.0 & 0.0 & 0.0 & 0.0 & 0.0 \\
\hline 0.0 & 0.0 & 0.0 & 0.0 & 0.0 & 0.0 & 0.0 & 0.0 \\
\hline 0.0 & 0.0 & 0.0 & 0.0 & 0.0 & 0.0 & 0.0 & 0.0 \\
\hline 0.0 & 0.0 & 0.0 & 0.0 & 0.0 & 0.0 & 0.0 & 0.0 \\
\hline 0.0 & 0.0 & 0.0 & 0.0 & 0.0 & 0.0 & 0.0 & 0.0 \\
\hline 0.0 & 0.0 & 0.0 & 0.0 & 0.0 & 0.0 & 0.0 & 0.0 \\
\hline 0.0 & 0.0 & 0.0 & 0.0 & 0.0 & 0.0 & 0.0 & 0.0 \\
\hline 0.0 & 0.0 & 0.0 & 0.0 & 0.0 & 0.0 & 0.0 & 0.0 \\
\hline 0.0 & 0.0 & 0.0 & 0.0 & 0.0 & 0.0 & 0.0 & 0.0 \\
\hline 0.0 & 0.0 & 0.0 & 0.0 & 0.0 & 0.0 & 0.0 & 0.0 \\
\hline 0.0 & 0.0 & 0.0 & 0.0 & 0.0 & 0.0 & 0.0 & 0.0 \\
\hline 0.0 & 0.0 & 0.0 & 0.0 & 0.0 & 0.0 & 0.0 & 0.0 \\
\hline 0.0 & 0.0 & 0.0 & 0.0 & 0.0 & 0.0 & 0.0 & 0.0 \\
\hline 0.0 & 0.0 & 0.0 & 0.0 & 0.0 & 0.0 & 0.0 & 0.0 \\
\hline 0.0 & 0.0 & 0.0 & 0.0 & 0.0 & 0.0 & 0.0 & 0.0 \\
\hline 0.0 & 0.0 & 0.0 & 0.0 & 0.0 & 0.0 & 0.0 & 0.0 \\
\hline 0.0 & 0.0 & 0.0 & 0.0 & 0.0 & 0.0 & 0.0 & 0.0 \\
\hline 0.0 & 0.0 & 0.0 & 0.0 & 0.0 & 0.0 & 0.0 & 0.0 \\
\hline 0.0 & 0.0 & 0.0 & 0.0 & 0.0 & 0.0 & 0.0 & 0.0 \\
\hline 0.0 & 0.0 & 0.0 & 0.0 & 0.0 & 0.0 & 0.0 & 0.0 \\
\hline 0.0 & 0.0 & 0.0 & 0.0 & 0.0 & 0.0 & 0.0 & 0.0 \\
\hline 0.0 & 0.0 & 0.0 & 0.0 & 0.0 & 0.0 & 0.0 & 0.0 \\
\hline 0.0 & 0.0 & 0.0 & 0.0 & 0.0 & 0.0 & 0.0 & 0.0 \\
\hline 0.0 & 0.0 & 0.0 & 0.0 & 0.0 & 0.0 & 0.0 & 0.0 \\
\hline 0.0 & 0.0 & 0.0 & 0.0 & $0: 0$ & 0.0 & 0.0 & 0.0 \\
\hline 0.0 & 0.0 & 0.0 & 0.0 & 0.0 & 0.0 & 0.0 & 0.0 \\
\hline 0.0 & 0.0 & 0.0 & 0.0 & 0.0 & 0.0 & 0.0 & 0.0 \\
\hline 0.0 & 0.0 & 0.0 & 0.0 & 0.0 & 0.0 & 0.0 & 0.0 \\
\hline 0.0 & 0.0 & 0.0 & 0.0 & 0.0 & 0.0 & 0.0 & 0.0 \\
\hline 0.0 & 0.0 & 0.0 & 0.0 & 0.0 & 0.0 & 0.0 & 0.0 \\
\hline
\end{tabular}




\begin{tabular}{|c|c|c|c|c|c|c|c|}
\hline 0.0 & 0.0 & 0.0 & 0.0 & 0.0 & 0.0 & 0.0 & 0.0 \\
\hline 0.0 & 0.0 & 0.0 & 0.0 & 0.0 & 0.0 & 0.0 & 0.0 \\
\hline 0.0 & 0.0 & 0.0 & 0.0 & 0.0 & 0.0 & 0.0 & 0.0 \\
\hline 0.0 & 0.0 & 0.0 & 0.0 & 0.0 & 0.0 & 0.0 & 0.0 \\
\hline 0.0 & 0.0 & 0.0 & 0.0 & 0.0 & 0.0 & 0.0 & 0.0 \\
\hline 0.0 & 0.0 & 0.0 & 0.0 & 0.0 & 0.0 & 0.0 & 0.0 \\
\hline 0.0 & 0.0 & 0.0 & 0.0 & 0.0 & 0.0 & 0.0 & 0.0 \\
\hline 0.0 & 0.0 & 0.0 & 0.0 & 0.0 & 0.0 & 0.0 & 0.0 \\
\hline 0.0 & 0.0 & 0.0 & 0.0 & 0.0 & 0.0 & 0.0 & 0.0 \\
\hline 0.0 & 0.0 & 0.0 & 0.0 & 0.0 & 0.0 & 0.0 & 0.0 \\
\hline 0.0 & 0.0 & 0.0 & 0.0 & 0.0 & 0.0 & 0.0 & 0.0 \\
\hline 0.0 & 0.0 & 0.0 & 0.0 & 0.0 & 0.0 & 0.0 & 0.0 \\
\hline 0.0 & 0.0 & 0.0 & 0.0 & 0.0 & 0.0 & 0.0 & 0.0 \\
\hline 0.0 & 0.0 & 0.0 & 0.0 & 0.0 & 0.0 & 0.0 & 0.0 \\
\hline 0.0 & 0.0 & 0.0 & 0.0 & 0.0 & 0.0 & 0.0 & 0.0 \\
\hline 0 & & & & & & & \\
\hline 0 & & & & & & & \\
\hline 0 & & & & & & & \\
\hline 0 & & & 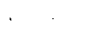 & & & & \\
\hline 1 & & & & & & & \\
\hline \multicolumn{8}{|c|}{ 1PRESCRIBED } \\
\hline 1 & 1 & $\because \quad 1$ & 1 & 1 & 1 & & \\
\hline 0.0 & 0.0 & 5927 & & & & & \\
\hline
\end{tabular}


APPENDIX C

INPUT DATA FOR THE TWO-DIMENSIONAL RECHARGE PROBLEM

2-DIMENSIONAL RECHARGE PROBLEM

VAUCLIN, KHANJI, AND VACHAUD; WRR 15(5); P. 1089-1101

C. DAVID UPDEGRAFF

PLOTPLOT

RUN

SINGLE

1.0
1.0
$1.0 \mathrm{E}-5$
1000
100
0.02
0.02
0.02
0.02
0.02
0.02
0.02
0.02
0.02
0.02
0.02
0.02
0.02
0.10
0.10
0.25
1800
3600
5400
7200
10800
14400
18000
21600
28800

SOIL

$1.0 \mathrm{E}-4$

1.0

0.64

0.48

0.32

0.16

0.00

$-0.16$

$-0.32$

$-0.48$

$-0.64$
1.0
1.0

1. $0 \mathrm{E}-5$

1000

18

0.02

0.02

0.02

0.02

0.02

0.02

0.02

0.02

0.02

0.02

0.02

0.02

0.02

0.10

0.10

0.25
4. $6 \mathrm{E}-6$

0.0

1. $0 \mathrm{E}-9$

0.0

0.02

0.02

0.02

0.02

0.02

0.02

0.02

0.02

0.02

0.02

0.02

0.02

0.02

0.10

0.25

PRINT

PRINT

PRINT

PRINT

PRINT

PRINT

PRINT

PRINT

PRINT
PLOT

PLOT

PLOT

PLOT

PLOT

PLOT

PLOT

PLOT

PLOT
0.0

0.0

0.0

0.0

0.02

0.02

0.02

0.02

0.02

0.02

0.02

0.02

0.02

0.02

0.02

0.02

0.10

0.25

0.10
0.25

0.02

0.02

0.10

0.02

0.02

0.02

0.02

0.02

0.02

0.02

0.02

0.02

0.02

0.02

MOISTURE

MOISTURE

MOISTURE

MOISTURE

MOISTURE

MOISTURE

MOISTURE

MOISTURE

MOISTURE 1

$\begin{array}{llllll}1 & 100 & 1 & 18 & 0 & 0\end{array}$

$\begin{array}{llll}0.3 & 9.7222 \mathrm{E}-5 & 9.7222 \mathrm{E}-5 & 9.7222 \mathrm{E}-5\end{array}$

$\begin{array}{ll}0.0 & 2.9\end{array}$

0.62

0.60

1.02 .5887062

0.46

0.44

0.58

0.56

0.42

0.40

0.26

0.24

0.14

0.12

0.10

0.08

$-0.02$

$-0.04$

$-0.06$

$-0.08$

$-0.18$

$-0.20$

$-0.22$

$-0.24$

$-0.36$

$-0.38$

$-0.40$

$-0.50$

$-0.52$

$-0.54$

$-0.56$

$-0.68$

$-0.70$

$-0.72$
0.54
0.38
0.22
0.06
$-0.10$
$-0.26$
$-0.42$
$-0.58$
$-0.74$

0.52

0.02

0.02

0.02

0.02

0.02

0.02

0.02

0.02

0.02

0.02

0.02

0.02

0.02

0.02

0.02

0.02

0.02

0.02

0.02

0.02

0.02

0.02

0.02

0.02

0.10

0.10

0.25

0.25

$-0.66$

0.36

0.50

0.04

0.18

$-0.12$

0.02

$-0.28$

$-0.14$

$-0.44$

$-0.30$

$-0.60$

$-0.46$

$-0.76$

$-0.62$

$-0.78$ 


\begin{tabular}{|c|c|c|c|c|c|c|c|}
\hline-0.80 & -0.82 & -0.84 & -0.86 & -0.88 & -0.90 & -0.92 & -0.94 \\
\hline-0.96 & -0.98 & -1.00 & -1.02 & -1.04 & -1.06 & -1.08 & -1.10 \\
\hline-1.12 & -1.14 & -1.16 & -1.18 & -1.20 & -1.22 & -1.24 & -1.26 \\
\hline-1.28 & -1.30 & -1.32 & -1.34 & & & & \\
\hline 0.64 & 0.62 & 0.60 & 0.58 & 0.56 & 0.54 & 0.52 & 0.5 \\
\hline 0.48 & 0.46 & 0.44 & 0.42 & 0.40 & 0.38 & 0.36 & 0.3 \\
\hline 0.32 & 0.30 & 0.28 & 0.26 & 0.24 & 0.22 & 0.20 & 0.1 \\
\hline 0.16 & 0.14 & 0.12 & 0.10 & 0.08 & 0.06 & 0.04 & 0.02 \\
\hline 0.00 & -0.02 & -0.04 & -0.06 & -0.08 & -0.10 & -0.12 & -0.14 \\
\hline-0.16 & -0.18 & -0.20 & -0.22 & -0.24 & -0.26 & -0.28 & -0.30 \\
\hline-0.32 & -0.34 & -0.36 & -0.38 & -0.40 & -0.42 & -0.44 & -0.46 \\
\hline-0.48 & -0.50 & -0.52 & -0.54 & -0.56 & -0.58 & -0.60 & -0.6 \\
\hline-0.64 & -0.66 & -0.68 & -0.70 & -0.72 & -0.74 & -0.76 & -0.7 \\
\hline-0.80 & -0.82 & -0.84 & -0.86 & -0.88 & -0.90 & -0.92 & -0.9 \\
\hline-0.96 & -0.98 & -1.00 & -1.02 & -1.04 & -1.06 & -1.08 & -1.1 \\
\hline-1.12 & -1.14 & -1.16 & -1.18 & -1.20 & -1.22 & -1.24 & -1.2 \\
\hline-1.28 & -1.30 & -1.32 & -1.34 & & & & \\
\hline 0.64 & 0.62 & 0.60 & 0.58 & 0.56 & 0.54 & 0.52 & 0.5 \\
\hline 0.48 & 0.46 & 0.44 & 0.42 & 0.40 & 0.38 & 0.36 & 0.34 \\
\hline 0.32 & 0.30 & 0.28 & 0.26 & 0.24 & 0.22 & 0.20 & 0.18 \\
\hline 0.16 & 0.14 & 0.12 & 0.10 & 0.08 & 0.06 & 0.04 & 0.02 \\
\hline 0.00 & -0.02 & -0.04 & -0.06 & -0.08 & -0.10 & -0.12 & -0.14 \\
\hline-0.16 & -0.18 & -0.20 & -0.22 & -0.24 & -0.26 & -0.28 & -0.3 \\
\hline-0.32 & -0.34 & -0.36 & -0.38 & -0.40 & -0.42 & -0.44 & -0.4 \\
\hline-0.48 & -0.50 & -0.52 & -0.54 & -0.56 & -0.58 & -0.60 & -0.6 \\
\hline-0.64 & -0.66 & -0.68 & -0.70 & -0.72 & -0.74 & -0.76 & -0.7 \\
\hline-0.80 & -0.82 & -0.84 & -0.86 & -0.88 & -0.90 & -0.92 & -0.9 \\
\hline-0.96 & -0.98 & -1.00 & -1.02 & -1.04 & -1.06 & -1.08 & -1.1 \\
\hline-1.12 & -1.14 & -1.16 & -1.18 & -1.20 & -1.22 & -1.24 & -1.2 \\
\hline-1.28 & -1.30 & -1.32 & -1.34 & & & & \\
\hline 0.64 & 0.62 & 0.60 & 0.58 & 0.56 & 0.54 & 0.52 & 0.5 \\
\hline 0.48 & 0.46 & 0.44 & 0.42 & 0.40 & 0.38 & 0.36 & 0.3 \\
\hline 0.32 & 0.30 & 0.28 & 0.26 & 0.24 & 0.22 & 0.20 & 0.1 \\
\hline 0.16 & 0.14 & 0.12 & 0.10 & 0.08 & 0.06 & 0.04 & 0.02 \\
\hline 0.00 & -0.02 & -0.04 & -0.06 & -0.08 & -0.10 & -0.12 & -0.1 \\
\hline-0.16 & -0.18 & -0.20 & -0.22 & -0.24 & -0.26 & -0.28 & -0.3 \\
\hline-0.32 & -0.34 & -0.36 & -0.38 & -0.40 & -0.42 & -0.44 & -0.4 \\
\hline-0.48 & -0.50 & -0.52 & -0.54 & -0.56 & -0.58 & -0.60 & -0.6 \\
\hline-0.64 & -0.66 & -0.68 & -0.70 & -0.72 & -0.74 & -0.76 & -0.7 \\
\hline-0.80 & -0.82 & -0.84 & -0.86 & -0.88 & -0.90 & -0.92 & -0.9 \\
\hline-0.96 & -0.98 & -1.00 & -1.02 & -1.04 & -1.06 & -1.08 & -1.1 \\
\hline-1.12 & -1.14 & -1.16 & -1.18 & -1.20 & -1.22 & -1.24 & -1.2 \\
\hline-1.28 & -1.30 & -1.32 & -1.34 & & & & \\
\hline 0.64 & 0.62 & 0.60 & 0.58 & 0.56 & 0.54 & 0.52 & 0.5 \\
\hline 0.48 & 0.46 & 0.44 & 0.42 & 0.40 & 0.38 & 0.36 & 0.3 \\
\hline 0.32 & 0.30 & 0.28 & 0.26 & 0.24 & 0.22 & 0.20 & 0.1 \\
\hline 0.16 & 0.14 & 0.12 & 0.10 & 0.08 & 0.06 & 0.04 & 0.0 \\
\hline 0.00 & -0.02 & -0.04 & -0.06 & -0.08 & -0.10 & -0.12 & -0.1 \\
\hline-0.16 & -0.18 & -0.20 & -0.22 & -0.24 & -0.26 & -0.28 & -0.3 \\
\hline-0.32 & -0.34 & -0.36 & -0.38 & -0.40 & -0.42 & -0.44 & -0.4 \\
\hline-0.48 & -0.50 & $=0.52$ & -0.54 & -0.56 & -0.58 & -0.60 & -0.6 \\
\hline-0.64 & -0.66 & -0.68 & -0.70 & -0.72 & -0.74 & -0.76 & -0.7 \\
\hline-0.80 & -0.82 & -0.84 & -0.86 & -0.88 & -0.90 & -0.92 & -0.9 \\
\hline-0.96 & -0.98 & -1.00 & -1.02 & -1.04 & -1.06 & -1.08 & -1.1 \\
\hline
\end{tabular}




\begin{tabular}{|c|c|c|c|c|c|c|c|}
\hline-1.12 & -1.14 & -1.16 & -1.18 & -1.20 & -1.22 & -1.24 & -1.26 \\
\hline-1.28 & -1.30 & -1.32 & $-1 . .34$ & & & & \\
\hline 0.64 & 0.62 & 0.60 & 0.58 & 0.56 & 0.54 & 0.52 & 0.50 \\
\hline 0.48 & 0.46 & 0.44 & 0.42 & 0.40 & 0.38 & 0.36 & 0.34 \\
\hline 0.32 & 0.30 & 0.28 & 0.26 & 0.24 & 0.22 & 0.20 & 0.18 \\
\hline 0.16 & 0.14 & 0.12 & 0.10 & 0.08 & 0.06 & 0.04 & 0.02 \\
\hline 0.00 & -0.02 & -0.04 & -0.06 & -0.08 & -0.10 & -0.12 & -0.14 \\
\hline-0.16 & -0.18 & -0.20 & -0.22 & -0.24 & -0.26 & -0.28 & -0.30 \\
\hline-0.32 & -0.34 & -0.36 & -0.38 & -0.40 & -0.42 & -0.44 & -0.46 \\
\hline-0.48 & -0.50 & -0.52 & -0.54 & -0.56 & -0.58 & -0.60 & -0.62 \\
\hline-0.64 & -0.66 & -0.68 & -0.70 & -0.72 & -0.74 & -0.76 & -0.78 \\
\hline-0.80 & -0.82 & -0.84 & -0.86 & -0.88 & -0.90 & -0.92 & -0.94 \\
\hline-0.96 & -0.98 & -1.00 & -1.02 & -1.04 & -1.06 & -1.08 & -1.10 \\
\hline-1.12 & -1.14 & -1.16 & -1.18 & -1.20 & -1.22 & -1.24 & -1.26 \\
\hline-1.28 & -1.30 & -1.32 & -1.34 & & & & \\
\hline 0.64 & 0.62 & 0.60 & 0.58 & 0.56 & 0.54 & 0.52 & 0.50 \\
\hline 0.48 & 0.46 & 0.44 & 0.42 & 0.40 & 0.38 & 0.36 & 0.34 \\
\hline 0.32 & 0.30 & 0.28 & 0.26 & 0.24 & 0.22 & 0.20 & 0.18 \\
\hline 0.16 & 0.14 & 0.12 & 0.10 & 0.08 & 0.06 & 0.04 & 0.02 \\
\hline 0.00 & -0.02 & -0.04 & -0.06 & -0.08 & -0.10 & -0.12 & -0.14 \\
\hline-0.16 & -0.18 & -0.20 & -0.22 & -0.24 & -0.26 & -0.28 & -0.30 \\
\hline-0.32 & -0.34 & -0.36 & -0.38 & -0.40 & -0.42 & -0.44 & -0.46 \\
\hline-0.48 & -0.50 & -0.52 & -0.54 & -0.56 & -0.58 & -0.60 & -0.62 \\
\hline-0.64 & -0.66 & -0.68 & -0.70 & -0.72 & -0.74 & -0.76 & -0.78 \\
\hline-0.80 & -0.82 & -0.84 & -0.86 & -0.88 & -0.90 & -0.92 & -0.94 \\
\hline-0.96 & -0.98 & -1.00 & -1.02 & -1.04 & -1.06 & -1.08 & -1.10 \\
\hline-1.12 & -1.14 & -1.16 & -1.18 & -1.20 & -1.22 & -1.24 & -1.26 \\
\hline-1.28 & -1.30 & -1.32 & -1.34 & & & & \\
\hline 0.64 & 0.62 & 0.60 & 0.58 & 0.56 & 0.54 & 0.52 & 0.50 \\
\hline 0.48 & 0.46 & 0.44 & 0.42 & 0.40 & 0.38 & 0.36 & 0.34 \\
\hline 0.32 & 0.30 & 0.28 & 0.26 & 0.24 & 0.22 & 0.20 & 0.18 \\
\hline 0.16 & 0.14 & 0.12 & 0.10 & 0.08 & 0.06 & 0.04 & 0.02 \\
\hline 0.00 & -0.02 & -0.04 & -0.06 & -0.08 & -0.10 & -0.12 & -0.14 \\
\hline-0.16 & -0.18 & -0.20 & -0.22 & -0.24 & -0.26 & -0.28 & -0.30 \\
\hline-0.32 & -0.34 & -0.36 & -0.38 & -0.40 & -0.42 & -0.44 & -0.46 \\
\hline-0.48 & -0.50 & -0.52 & -0.54 & -0.56 & -0.58 & -0.60 & -0.62 \\
\hline-0.64 & -0.66 & -0.68 & -0.70 & -0.72 & -0.74 & -0.76 & -0.78 \\
\hline-0.80 & -0.82 & -0.84 & -0.86 & -0.88 & -0.90 & -0.92 & -0.94 \\
\hline-0.96 & -0.98 & -1.00 & -1.02 & -1.04 & -1.06 & -1.08 & -1.10 \\
\hline-1.12 & -1.14 & -1.16 & -1.18 & -1.20 & -1.22 & -1.24 & -1.26 \\
\hline-1.28 & -1.30 & -1.32 & -1.34 & & & & \\
\hline 0.64 & 0.62 & 0.60 & 0.58 & 0.56 & 0.54 & 0.52 & 0.50 \\
\hline 0.48 & 0.46 & 0.44 & 0.42 & 0.40 & 0.38 & 0.36 & 0.34 \\
\hline 0.32 & 0.30 & 0.28 & 0.26 & 0.24 & 0.22 & 0.20 & 0.18 \\
\hline 0.16 & 0.14 & 0.12 & 0.10 & 0.08 & 0.06 & 0.04 & 0.02 \\
\hline 0.00 & -0.02 & -0.04 & -0.06 & -0.08 & -0.10 & -0.12 & -0.14 \\
\hline-0.16 & -0.18 & -0.20 & -0.22 & -0.24 & -0.26 & -0.28 & -0.30 \\
\hline-0.32 & -0.34 & -0.36 & -0.38 & -0.40 & -0.42 & -0.44 & -0.46 \\
\hline-0.48 & -0.50 & -0.52 & -0.54 & -0.56 & -0.58 & -0.60 & -0.62 \\
\hline-0.64 & -0.66 & -0.68 & -0.70 & -0.72 & -0.74 & -0.76 & -0.78 \\
\hline-0.80 & -0.82 & -0.84 & -0.86 & -0.88 & -0.90 & -0.92 & -0.94 \\
\hline-0.96 & -0.98 & -1.00 & -1.02 & -1.04 & -1.06 & -1.08 & -1.10 \\
\hline-1.12 & -1.14 & -1.16 & -1.18 & -1.20 & -1.22 & -1.24 & -1.26 \\
\hline-1.28 & -1.30 & -1.32 & -1.34 & & & & \\
\hline
\end{tabular}




\begin{tabular}{|c|c|c|c|c|c|c|c|}
\hline 0.64 & 0.62 & 0.60 & 0.58 & 0.56 & 0.54 & 0.52 & 0.50 \\
\hline 0.48 & 0.46 & 0.44 & 0.42 & 0.40 & 0.38 & 0.36 & 0.34 \\
\hline 0.32 & 0.30 & 0.28 & 0.26 & 0.24 & 0.22 & 0.20 & 0.18 \\
\hline 0.16 & 0.14 & 0.12 & 0.10 & 0.08 & 0.06 & 0.04 & 0.02 \\
\hline 0.00 & -0.02 & -0.04 & -0.06 & -0.08 & -0.10 & -0.12 & -0.14 \\
\hline-0.16 & -0.18 & -0.20 & -0.22 & -0.24 & -0.26 & -0.28 & -0.30 \\
\hline-0.32 & -0.34 & -0.36 & -0.38 & -0.40 & -0.42 & -0.44 & -0.46 \\
\hline-0.48 & -0.50 & -0.52 & -0.54 & -0.56 & -0.58 & -0.60 & -0.62 \\
\hline-0.64 & -0.66 & -0.68 & -0.70 & -0.72 & -0.74 & -0.76 & -0.78 \\
\hline-0.80 & -0.82 & -0.84 & -0.86 & -0.88 & -0.90 & -0.92 & -0.94 \\
\hline-0.96 & -0.98 & -1.00 & -1.02 & -1.04 & -1.06 & -1.08 & -1.10 \\
\hline-1.12 & -1.14 & -1.16 & -1.18 & -1.20 & -1.22 & -1.24 & -1.26 \\
\hline-1.28 & -1.30 & -1.32 & -1.34 & & & & \\
\hline 0.64 & 0.62 & 0.60 & 0.58 & 0.56 & 0.54 & 0.52 & 0.50 \\
\hline 0.48 & 0.46 & 0.44 & 0.42 & 0.40 & 0.38 & 0.36 & 0.34 \\
\hline 0.32 & 0.30 & 0.28 & 0.26 & 0.24 & 0.22 & 0.20 & 0.18 \\
\hline 0.16 & 0.14 & 0.12 & 0.10 & 0.08 & 0.06 & 0.04 & 0.02 \\
\hline 0.00 & -0.02 & -0.04 & -0.06 & -0.08 & -0.10 & -0.12 & -0.14 \\
\hline-0.16 & -0.18 & -0.20 & -0.22 & -0.24 & -0.26 & -0.28 & -0.30 \\
\hline-0.32 & -0.34 & -0.36 & -0.38 & -0.40 & -0.42 & -0.44 & -0.46 \\
\hline-0.48 & -0.50 & -0.52 & -0.54 & -0.56 & -0.58 & -0.60 & -0.62 \\
\hline-0.64 & -0.66 & -0.68 & -0.70 & -0.72 & -0.74 & -0.76 & -0.78 \\
\hline-0.80 & -0.82 & -0.84 & -0.86 & -0.88 & -0.90 & -0.92 & -0.94 \\
\hline-0.96 & -0.98 & -1.00 & -1.02 & -1.04 & -1.06 & -1.08 & -1.10 \\
\hline-1.12 & -1.14 & -1.16 & -1.18 & -1.20 & -1.22 & -1.24 & -1.26 \\
\hline-1.28 & -1.30 & -1.32 & -1.34 & & & & \\
\hline 0.64 & 0.62 & 0.60 & 0.58 & 0.56 & 0.54 & 0.52 & 0.50 \\
\hline 0.48 & 0.46 & 0.44 & 0.42 & 0.40 & 0.38 & 0.36 & 0.34 \\
\hline 0.32 & 0.30 & 0.28 & 0.26 & 0.24 & 0.22 & 0.20 & 0.18 \\
\hline 0.16 & 0.14 & 0.12 & 0.10 & 0.08 & 0.06 & 0.04 & 0.02 \\
\hline 0.00 & -0.02 & -0.04 & -0.06 & -0.08 & -0.10 & -0.12 & -0.14 \\
\hline-0.16 & -0.18 & -0.20 & -0.22 & -0.24 & -0.26 & -0.28 & -0.30 \\
\hline-0.32 & -0.34 & -0.36 & -0.38 & -0.40 & -0.42 & -0.44 & -0.46 \\
\hline-0.48 & -0.50 & -0.52 & -0.54 & -0.56 & -0.58 & -0.60 & -0.62 \\
\hline-0.64 & -0.66 & -0.68 & -0.70 & -0.72 & -0.74 & -0.76 & -0.78 \\
\hline-0.80 & -0.82 & -0.84 & -0.86 & -0.88 & -0.90 & -0.92 & -0.94 \\
\hline-0.96 & -0.98 & -1.00 & -1.02 & -1.04 & -1.06 & -1.08 & -1.10 \\
\hline $\begin{array}{l}-1.12 \\
-1.28\end{array}$ & $\begin{array}{l}-1.14 \\
-1.30\end{array}$ & $\begin{array}{l}-1.16 \\
-1.32\end{array}$ & $\begin{array}{l}-1.18 \\
-1.34\end{array}$ & -1.20 & -1.22 & -1.24 & -1.26 \\
\hline 0.64 & 0.62 & 0.60 & 0.58 & 0.56 & 0.54 & 0.52 & 0.50 \\
\hline 0.48 & 0.46 & 0.44 & 0.42 & 0.40 & 0.38 & 0.36 & 0.34 \\
\hline 0.32 & 0.30 & 0.28 & 0.26 & 0.24 & 0.22 & 0.20 & 0.18 \\
\hline 0.16 & 0.14 & 0.12 & 0.10 & 0.08 & 0.06 & 0.04 & 0.02 \\
\hline 0.00 & -0.02 & -0.04 & -0.06 & $=0.08$ & -0.10 & -0.12 & -0.14 \\
\hline-0.16 & -0.18 & -0.20 & -0.22 & -0.24 & -0.26 & -0.28 & -0.30 \\
\hline-0.32 & -0.34 & -0.36 & -0.38 & -0.40 & -0.42 & -0.44 & -0.46 \\
\hline-0.48 & -0.50 & -0.52 & -0.54 & -0.56 & -0.58 & -0.60 & -0.62 \\
\hline-0.64 & -0.66 & -0.68 & -0.70 & -0.72 & -0.74 & -0.76 & -0.78 \\
\hline-0.80 & -0.82 & -0.84 & -0.86 & -0.88 & -0.90 & -0.92 & -0.94 \\
\hline-0.96 & -0.98 & -1.00 & -1.02 & -1.04 & -1.06 & -1.08 & -1.10 \\
\hline-1.12 & -1.14 & -1.16 & -1.18 & -1.20 & -1.22 & -1.24 & -1.26 \\
\hline-1.28 & -1.30 & -1.32 & -1.34 & & & & \\
\hline 0.64 & 0.62 & 0.60 & 0.58 & 0.56 & 0.54 & 0.52 & 0.50 \\
\hline 0.48 & 0.46 & 0.44 & 0.42 & 0.40 & 0.38 & 0.36 & 0.34 \\
\hline
\end{tabular}




\begin{tabular}{|c|c|c|c|c|c|c|c|}
\hline 0.32 & 0.30 & 0.28 & 0.26 & 0.24 & 0.22 & 0.20 & 0.18 \\
\hline 0.16 & 0.14 & 0.12 & 0.10 & 0.08 & 0.06 & 0.04 & 0.02 \\
\hline 0.00 & -0.02 & -0.04 & -0.06 & -0.08 & -0.10 & -0.12 & -0.14 \\
\hline-0.16 & -0.18 & -0.20 & -0.22 & -0.24 & -0.26 & -0.28 & -0.30 \\
\hline-0.32 & -0.34 & -0.36 & -0.38 & -0.40 & -0.42 & -0.44 & -0.46 \\
\hline-0.48 & -0.50 & -0.52 & -0.54 & -0.56 & -0.58 & -0.60 & -0.62 \\
\hline-0.64 & -0.66 & -0.68 & -0.70 & -0.72 & -0.74 & -0.76 & -0.78 \\
\hline-0.80 & -0.82 & -0.84 & -0.86 & -0.88 & -0.90 & -0.92 & -0.94 \\
\hline-0.96 & -0.98 & -1.00 & -1.02 & -1.04 & -1.06 & -1.08 & -1.10 \\
\hline-1.12 & -1.14 & -1.16 & -1.18 & -1.20 & -1.22 & -1.24 & -1.26 \\
\hline-1.28 & -1.30 & -1.32 & -1.34 & & & & \\
\hline 0.64 & 0.62 & 0.60 & 0.58 & 0.56 & 0.54 & 0.52 & 0.50 \\
\hline 0.48 & 0.46 & 0.44 & 0.42 & 0.40 & 0.38 & 0.36 & 0.34 \\
\hline 0.32 & 0.30 & 0.28 & 0.26 & 0.24 & 0.22 & 0.20 & 0.18 \\
\hline 0.16 & 0.14 & 0.12 & 0.10 & 0.08 & 0.06 & 0.04 & 0.02 \\
\hline 0.00 & -0.02 & -0.04 & -0.06 & -0.08 & -0.10 & -0.12 & -0.14 \\
\hline-0.16 & -0.18 & -0.20 & -0.22 & -0.24 & -0.26 & -0.28 & -0.30 \\
\hline-0.32 & -0.34 & -0.36 & -0.38 & -0.40 & -0.42 & -0.44 & -0.46 \\
\hline-0.48 & -0.50 & -0.52 & -0.54 & -0.56 & -0.58 & -0.60 & -0.62 \\
\hline-0.64 & -0.66 & -0.68 & -0.70 & -0.72 & -0.74 & -0.76 & -0.78 \\
\hline-0.80 & -0.82 & -0.84 & -0.86 & -0.88 & -0.90 & -0.92 & -0.94 \\
\hline-0.96 & -0.98 & -1.00 & -1.02 & -1.04 & -1.06 & -1.08 & -1.10 \\
\hline-1.12 & -1.14 & -1.16 & -1.18 & -1.20 & -1.22 & -1.24 & -1.26 \\
\hline-1.28 & -1.30 & -1.32 & -1.34 & & & & \\
\hline 0.64 & 0.62 & 0.60 & 0.58 & 0.56 & 0.54 & 0.52 & 0.50 \\
\hline 0.48 & 0.46 & 0.44 & 0.42 & 0.40 & 0.38 & 0.36 & 0.34 \\
\hline 0.32 & 0.30 & 0.28 & 0.26 & 0.24 & 0.22 & 0.20 & 0.18 \\
\hline 0.16 & 0.14 & 0.12 & 0.10 & 0.08 & 0.06 & 0.04 & 0.02 \\
\hline 0.00 & -0.02 & -0.04 & -0.06 & -0.08 & -0.10 & -0.12 & -0.14 \\
\hline-0.16 & -0.18 & -0.20 & -0.22 & -0.24 & -0.26 & -0.28 & -0.30 \\
\hline-0.32 & -0.34 & -0.36 & -0.38 & -0.40 & -0.42 & -0.44 & -0.46 \\
\hline-0.48 & -0.50 & -0.52 & -0.54 & -0.56 & -0.58 & -0.60 & -0.62 \\
\hline-0.64 & -0.66 & -0.68 & -0.70 & -0.72 & -0.74 & -0.76 & -0.78 \\
\hline-0.80 & -0.82 & -0.84 & -0.86 & -0.88 & -0.90 & -0.92 & -0.94 \\
\hline-0.96 & -0.98 & -1.00 & -1.02 & -1.04 & -1.06 & -1.08 & -1.10 \\
\hline-1.12 & -1.14 & -1.16 & -1.18 & -1.20 & -1.22 & -1.24 & -1.26 \\
\hline-1.28 & -1.30 & -1.32 & -1.34 & & & & \\
\hline 0.64 & 0.62 & 0.60 & 0.58 & 0.56 & 0.54 & 0.52 & 0.50 \\
\hline 0.48 & 0.46 & 0.44 & 0.42 & 0.40 & 0.38 & 0.36 & 0.34 \\
\hline 0.32 & 0.30 & 0.28 & 0.26 & 0.24 & 0.22 & 0.20 & 0.18 \\
\hline 0.16 & 0.14 & 0.12 & 0.10 & 0.08 & 0.06 & 0.04 & 0.02 \\
\hline 0.00 & -0.02 & -0.04 & -0.06 & -0.08 & -0.10 & -0.12 & -0.14 \\
\hline-0.16 & -0.18 & -0.20 & -0.22 & -0.24 & -0.26 & -0.28 & -0.30 \\
\hline-0.32 & -0.34 & -0.36 & -0.38 & -0.40 & -0.42 & -0.44 & -0.46 \\
\hline-0.48 & -0.50 & -0.52 & -0.54 & -0.56 & -0.58 & -0.60 & -0.62 \\
\hline-0.64 & -0.66 & -0.68 & -0.70 & -0.72 & -0.74 & -0.76 & -0.78 \\
\hline-0.80 & -0.82 & -0.84 & -0.86 & -0.88 & -0.90 & -0.92 & -0.94 \\
\hline-0.96 & -0.98 & -1.00 & -1.02 & -1.04 & -1.06 & -1.08 & -1.10 \\
\hline-1.12 & -1.14 & -1.16 & -1.18 & -1.20 & -1.22 & -1.24 & -1.26 \\
\hline-1.28 & -1.30 & -1.32 & -1.34 & & & & \\
\hline 0.64 & 0.62 & 0.60 & 0.58 & 0.56 & 0.54 & 0.52 & 0.50 \\
\hline 0.48 & 0.46 & 0.44 & 0.42 & 0.40 & 0.38 & 0.36 & 0.34 \\
\hline 0.32 & 0.30 & 0.28 & 0.26 & 0.24 & 0.22 & 0.20 & 0.18 \\
\hline 0.16 & 0.14 & 0.12 & 0.10 & 0.08 & 0.06 & 0.04 & 0.02 \\
\hline
\end{tabular}




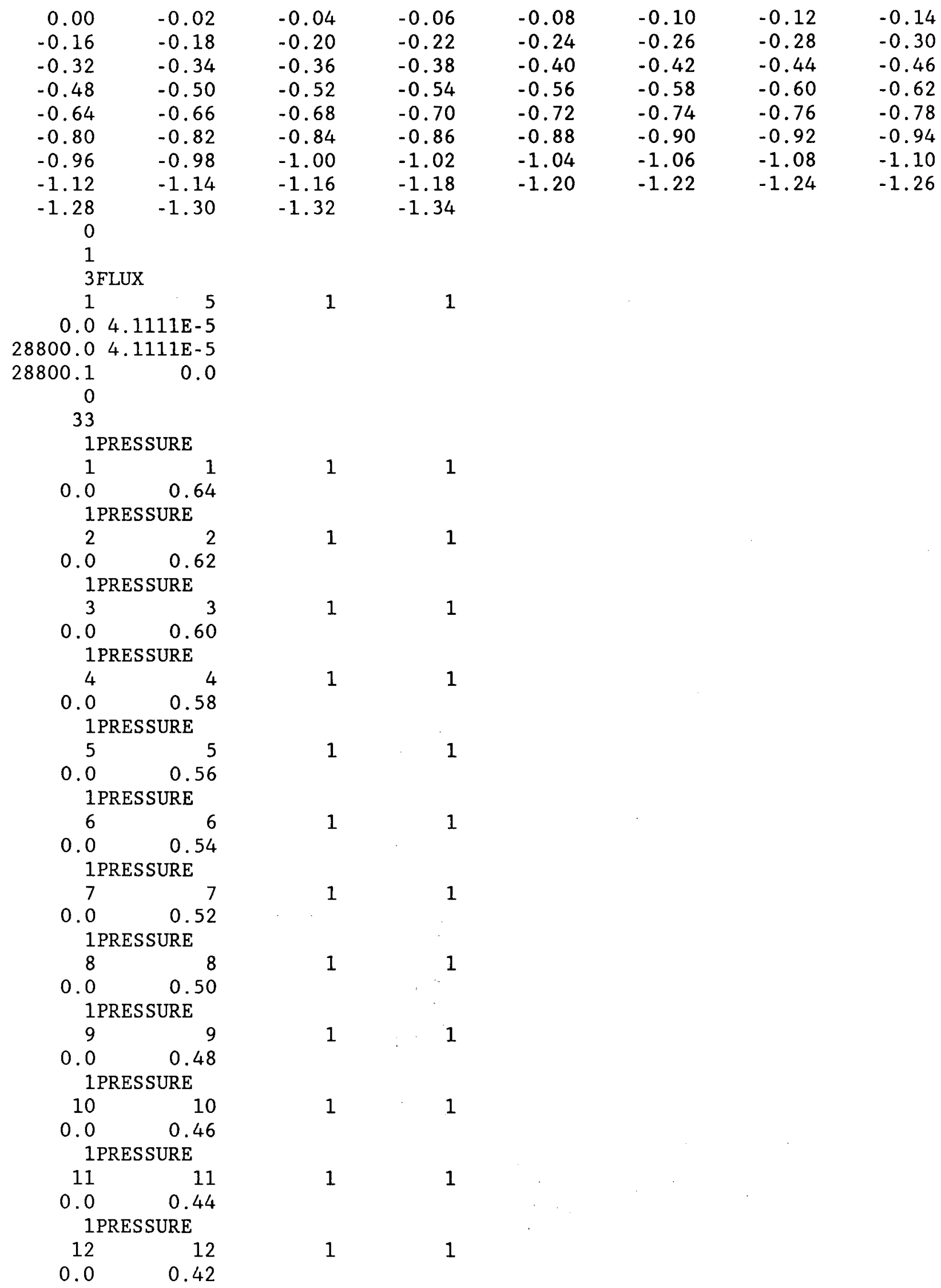




\begin{tabular}{|c|c|c|c|}
\hline \multicolumn{2}{|c|}{ 1PRESSURE } & \multirow{3}{*}{1} & \multirow[b]{2}{*}{1} \\
\hline 13 & 13 & & \\
\hline 0.0 & 0.40 & & \\
\hline \multicolumn{2}{|c|}{ 1PRESSURE } & \multirow{3}{*}{1} & \multirow{3}{*}{1} \\
\hline 14 & 14 & & \\
\hline 0.0 & 0.38 & & \\
\hline \multicolumn{2}{|c|}{ 1PRESSURE } & \multirow{3}{*}{1} & \multirow{3}{*}{1} \\
\hline 15 & 15 & & \\
\hline 0.0 & 0.36 & & \\
\hline \multicolumn{2}{|c|}{ 1PRESSURE } & \multirow{3}{*}{1} & \multirow{3}{*}{1} \\
\hline 16 & 16 & & \\
\hline 0.0 & 0.34 & & \\
\hline \multicolumn{2}{|c|}{ 1PRESSURE } & \multirow{3}{*}{1} & \multirow{3}{*}{1} \\
\hline 17 & 17 & & \\
\hline 0.0 & 0.32 & & \\
\hline \multicolumn{2}{|c|}{ 1PRESSURE } & \multirow{3}{*}{1} & \multirow{3}{*}{1} \\
\hline 18 & 18 & & \\
\hline 0.0 & 0.30 & & \\
\hline \multicolumn{2}{|c|}{ 1PRESSURE } & \multirow{3}{*}{1} & \\
\hline 19 & 19 & & 1 \\
\hline 0.0 & 0.28 & & \\
\hline & JRE & & \\
\hline 20 & 20 & 1 & 1 \\
\hline 0.0 & 0.26 & & \\
\hline & JRE & & \\
\hline 21 & 21 & 1 & 1 \\
\hline 0.0 & 0.24 & & \\
\hline & JRE & & \\
\hline 22 & 22 & 1 & 1 \\
\hline 0.0 & 0.22 & & \\
\hline & JRE & & \\
\hline 23 & 23 & 1 & 1 \\
\hline 0.0 & 0.20 & & \\
\hline & JRE & & \\
\hline 24 & 24 & 1 & 1. \\
\hline 0.0 & 0.18 & & \\
\hline & JRE & & \\
\hline 25 & 25 & 1 & 1 \\
\hline 0.0 & 0.16 & & \\
\hline & JRE & & \\
\hline 26 & 26 & 1 & 1 \\
\hline 0.0 & 0.14 & & \\
\hline & JRE & & \\
\hline 27 & 27 & 1 & 1 \\
\hline 0.0 & 0.12 & & \\
\hline & JRE & & \\
\hline 28 & 28 & 1 & 1 \\
\hline 0.0 & 0.10 & & \\
\hline & JRE & & \\
\hline 29 & 29 & 1 & 1 \\
\hline 0.0 & 0.08 & & \\
\hline & JRE & & \\
\hline 30 & 30 & 1 & 1 \\
\hline 0.0 & 0.06 & & \\
\hline
\end{tabular}


1PRESSURE

31

$0.0 \quad 0.04$

1PRESSURE

$32 \quad 32$

$0.0 \quad 0.02$

1PRESSURE

$33 \quad 33$

$0.0 \quad 0.00$

11

11

11

0

- Page 133 - 

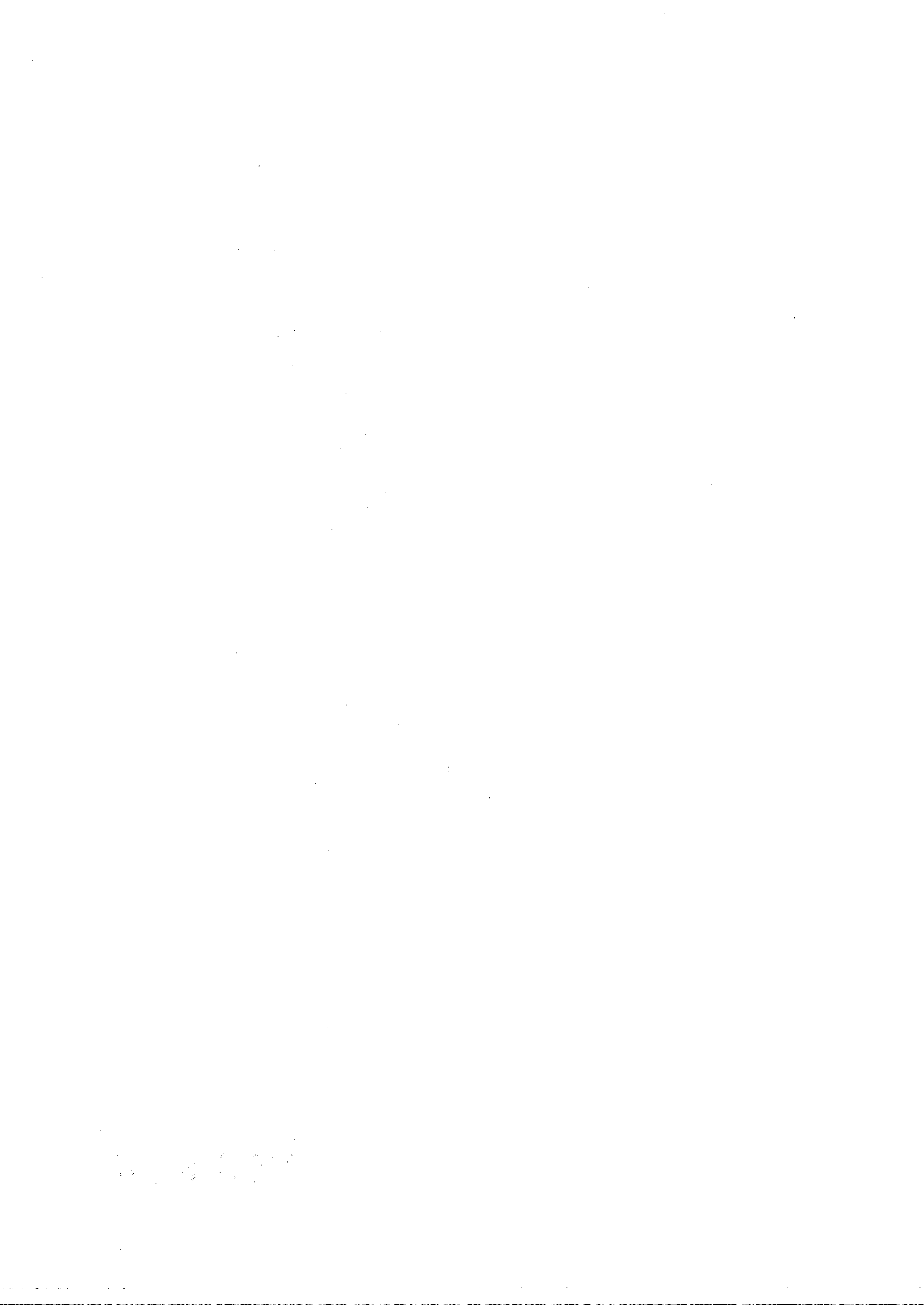
(RESEARCH)

G. F. Birchard

MS NL/S-260

U. S. Nuclear Regulatory

Commission

Washington, DC 20555

Linda Kovach

MS NL/S - 260

U. S. Nuclear Regulatory

Commission

Washington, DC 20555

Tim Margulies

MS NL/S - 260

U. S. Nuclear Regulatory

Commission

Washington, DC 20555

T. J. McCartin

MS NL/S - 260

U. S. Nuclear Regulatory

Commission

Washington, DC 20555

T. J. Nicholson

MS NL/S-260

U. S. Nuclear Regulatory

Commission

Washington, DC 20555

W. Ott

MS NL/S-260

U. S. Nuclear Regulatory

Commission

Washington, DC 20555

J. D. Randa11

MS NL/S-260

U. S. Nuclear Regulatory

Commission

Washington, DC 20555

R.L. Shephard

MS NL/S-007

U. S. Nuclear Regulatory

Commission

Washington, DC 20555
Mel Silberberg

MS NL/S-260

U. S. Nuclear Regulatory

Commission

Washington, DC 20555

(NMSS HLW)

P. Altomare

MS 4-H-3

U. S. Nuclear Regulatory

Commission

Washington, DC 20555

Ronald Ballard

MS 4- $\mathrm{H}-3$

U. S. Nuclear Regulatory

Commission

Washington, DC 20555

John Bradbury

MS $4-\mathrm{H}-3$

U. S. Nuclear Regulatory

Commission

Washington, DC 20555

Pauline Brooks

MS 4-H-3

U. S. Nuclear Regulatory

Commission

Washington, DC 20555

K.C. Chang

MS 4-H-3

U. S. Nuclear Regulatory

Commission

Washington, DC 20555

Donald L. Chery, Jr.

MS 4-H-3

U. S. Nuclear Regulatory

Commission

Washington, DC 20555

R. B. Code 11

MS 4- $\mathrm{H}-3$

U. S. Nuclear Regulatory

Commission

Washington, DC 20555

\section{DO NOT MICROFILM \\ -Page 135-


Neil Coleman

MS 4- $\mathrm{H}-3$

U. S. Nuclear Regulatory

Commission

Washington, DC 20555

Seth Coplan

MS 4-H-3

U. S. Nuclear Regulatory

Commission

Washington, DC 20555

Norman A. Eisenberg

MS $4-\mathrm{H}-3$

U. S. Nuclear Regulatory

Commission

Washington, DC 20555

D.J. Fehringer

MS 4- $\mathrm{H}-3$

U. S. Nuclear Regulatory

Commission

Washington, DC 20555

William Ford

MS 4-H-3

U. S. Nuclear Regulatory

Commission

Washington, DC 20555

P. S. Justus

MS 4-H-3

U. S. Nuclear Regulatory

Commission

Washington, DC 20555

Keith McConnel1

MS 4- $\mathrm{H}-3$

U. S. Nuclear Regulatory

Commission

Washington, DC 20555

Tin Mo

MS 4- $\mathrm{H}-3$

U. S. Nuclear Regulatory

Commission

Washington, DC 20555

M.S. Nataraja

MS 4-H-3

U. S. Nuclear Regulatory

Commission

Washington, DC 20555
Jim Park

MS 4-H-3

U. S. Nuclear Regulatory

Commission

Washington, DC 20555

J.J . Peshel

MS 4-H-3

U. S. Nuclear Regulatory

Commission

Washington, DC 20555

J . Pohle

MS 4-H-3

U. S. Nuclear Regulatory

Commission

Washington, DC 20555

John Trapp

MS 4-H-3

U. S. Nuclear Regulatory

Commission

Washington, DC 20555

B.J . Youngblood

MS 4-H-3

U. S. Nuclear Regulatory

Commission

Washington, DC 20555

(ADDITIONAL)

D. Alexander

U.S. Department of Energy

Regulatory Compliance Branch

Office of Civilian Radioactive

Waste Management

1000 Independence Avenue

Washington, DC 20585

A. Alonso-Santos

Universidad Politecnica de Madrid

J. Gutierrez Abascal, 2

28008 Madrid, SPAIN

George Apostolakis

University of California

5532 Boelter Hall

Los Angeles, CA 90024

DO NOT MICROFILM -Page 136 -

\section{THIS PAGE}


D.E. Billington

Harwell Laboratory

United Kingdom Atomic Energy

Authority

Oxfordshire OX11 ORA

UNITED KINGDOM

J. Boak

U.S. Department of Energy

Yucca Mountain Project Office

P.O. Box 98518

Las Vegas, NV 89193-8518

R.L. Bras

Ralph Parsons Laboratory

Dept. of Civil Engineering

Mass. Institute of Technology

Cambridge, MA 02139

Douglas Brosseau

ERCE, Inc.

1717 Louisiana NE, Suite 202

Albuquerque, NM 87110

P. Carboneras-Martinez

ENRESA

Calle Emilio Vargas, 7

28043 Madrid, SPAIN

C. Cole

Battelle Pacific Northwest

Laboratories

3100 Port of Benton

Sigma $V$ Building

Richland, WA 99352

J. J. K. Daemen

Department Mining and Geological

Engineering

University of Arizona

Tucson, AZ 85721

G. de Marsily

Ecole des mines de Paris

35, Rue Saint-Honore

77305 Fontainbleau

FRANCE

P. Dickman

U.S. Department of Energy

Nevada Operations Office

P.O. Box 98518

Las Vegas, NV 89193-8518
P.G. Doctor

Battelle Pacific Northwest

Laboratories

3100 Port of Benton

Sigma $V$ Building

Rlchland, WA 99352

Frank Dodge

Center for Nuclear Waste

Regulatory Analyses

Southwest Research Institute

6220 Culebra Rd.

San Antonio, TX 78228-0510

J.R. Dyer

U.S. Department of Energy

Yucca Mountain Project Office

P.0. Box 98518

Las Vegas, NV 89193-8518

P. Eslinger

Battelle Pacific Northwest

Laboratories

3100 Port of Benton

Sigma $\mathrm{V}$ Building

Richland, WA 99352

D. D. Evans

Department of Hydrology and Water

Resources

University of Arizona

Tucson, AZ 85721

Christopher Frietas

Center for Nuclear Waste

Regulatory Analyses

Southwest Research Institute

6220 Culebra Rd.

San Antonio, TX 78228-0510

Katherine N. Gaither

Applied Research Technical

Services, Inc.

4300 San Mateo NE, Suite A220

Albuquerque, NM 87110

D. Galson

Nuclear Energy Agency

Organization for Economic

Cooperation

and Development

38, Boulevard Suchet

F-75016 Paris, FRANCE

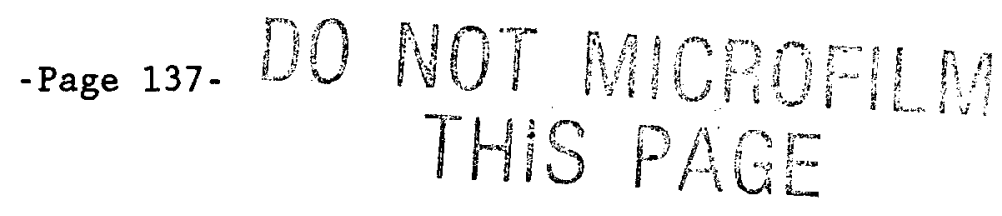


Professor Lynn W. Gelhar

MIT

Department of Civil Engineering

Room 48-329

Cambridge, MA 02139

\section{S. Gomberg}

U.S. Department of Energy

Regulatory Compliance Branch Office of Civilian Radioactive

Waste Management

1000 Independence Avenue

Washington, DC 20585

Michael T. Goodrich

GRAM, Inc.

1709 Moon St., NE

Albuquerque, NM 87112

Ronald T. Green

Center for Nuclear Waste

Regulatory Analyses

Southwest Research Institute

6220 Culebra Rd.

San Antonio, TX 78228-0510

A.L. Gutjahr

Department of Mathematics

New Mexico Institute of Mining

and Technology

Socorro, NM 87801

Sharif Heger

Department of Chemical and

Nuclear Engineering

University of New Mexico

Albuquerque, NM 87131

D.P. Hodgkinson

INTERA - ECL

Chiltern House

45 Station Road

Henley-on-Thames

Oxfordshire, RG9 1 AT

UNITED KINGDOM

Francis A. Kulacki

Office of the Dean

College of Engineering

Colorado State University

Fort Collins, CO 80523
Clarence E. Lee

Applied Physics, Inc.

5353 Wyoming Blvd., NE, Suite 3

Albuquerque, NM 87109

A.M. Liebetrau

Battelle Pacific Northwest

Laboratories

3100 Port of Benton

Sigma V Building

Richland, WA 99352

D.E. Longs ine

INTERA Environmental Consultants

Inc.

$6850^{\circ}$ Austin Center Blvd.

Suite 300

Austin, TX 78731

Sven 0 , Magnuson

$E$ G \& G Idaho, Inc.

P.0. Box 1625

Idaho Falls, Idaho 83415

Dennis McLaugh1in

MIT

Department of Civil Engineering

Room 48-329

Cambridge, MA 02139

Prasad K. Nair

Center for Nuclear Waste

Regulatory Analyses

Southwest Research Institute

6220 Culebra Rd.

San Antonio, TX 78228-0510

Shlomo P. Neuman

Department of Hydrology and Water

Resources

University of Arizona

Tucson, Arizona 85721

Gretchen C. Newman

GRAM, Inc.

1709 Moon St., NE

Albuquerque, NM 87112

A. Nies

Institut fur Tieflagerung

Theodor-Heuss-Strass 4

D-3300 Braunschweig

FEDERAL REPUBLIC OF GERMANY

DO NOT MICROFILM -Page 138- 
Wesley C. Patrick

Center for Nuclear Waste

Regulatory Analyses

Southwest Research Institute

6220 Culebra Rd.

San Antonio, TX 78228-0510

P. Prado-Herrero

CIEMAT

Centro de Investigaciones

Energeticas Medioambientales

y Tecnologicas

Avda. Complutense, 22

28040 Madrid, SPAIN

Todd C. Rasmussen

Department of Hydrology and

Water Resources

University of Arizona

Tucson, AZ 85721

M. Reeves

INTERA Environmental Consultants

Inc.

6850 Austin Center Blvd.

Suite 300

Austin, TX 78731

Judith A. Rollstin

GRAM, Inc.

1709 Moon St., NE

Albuquerque, NM 87112

John Russel1

Center for Nuclear Waste

Regulatory Analyses

Southwest Research Institute

6220 Culebra Rd.

San Antonio, TX 78228-0510

Budhi Sagar

Center for Nuclear Waste

Regulatory Analyses

Southwest Research Institute

6220 Culebra Rd.

San Antonio, TX 78228-0510

S. L. Smith

WS Atkins Engineering Sciences

Woodcote Grove

Ashley Road

Epsom

Surrey KT18 5BW

UNITED KINGDOM
C. Thegerstrom

Nuclear Energy Agency

Organization for Economic

Cooperation

and Development

38, Boulevard Suchet

F-75016 Paris, FRANCE

B.G.J. Thompson

HM Inspectorate of Pollution

Romney House

43 Marsham Street

London SW1P 3PY

UNITED KINGDOM

Kenneth E. Torrance

Sibley School of Mechanical and

Aerospace Engineering

Upson and Grumman Halls

Corne11 University

Ithaca, NY 14850

C. David Updegraff

GRAM, Inc.

1709 Moon St., NE

Albuquerque, NM 87112

A.E. Van Luik

Battelle Pacific Northwest

Laboratories

3100 Port of Benton

Sigma V Building

Richland, WA 99352

T. Vieno

Technical Research Center of

Finland

P.O. Box 169

SF-00181 Helsinki 18

FINLAND

Krishan K. Wahi

GRAM, Inc.

1709 Moon St., NE

Albuquerque, NM

Tony Zimmerman

GRAM, Inc.

1709 Moon St., NE

Albuquerque, NM 


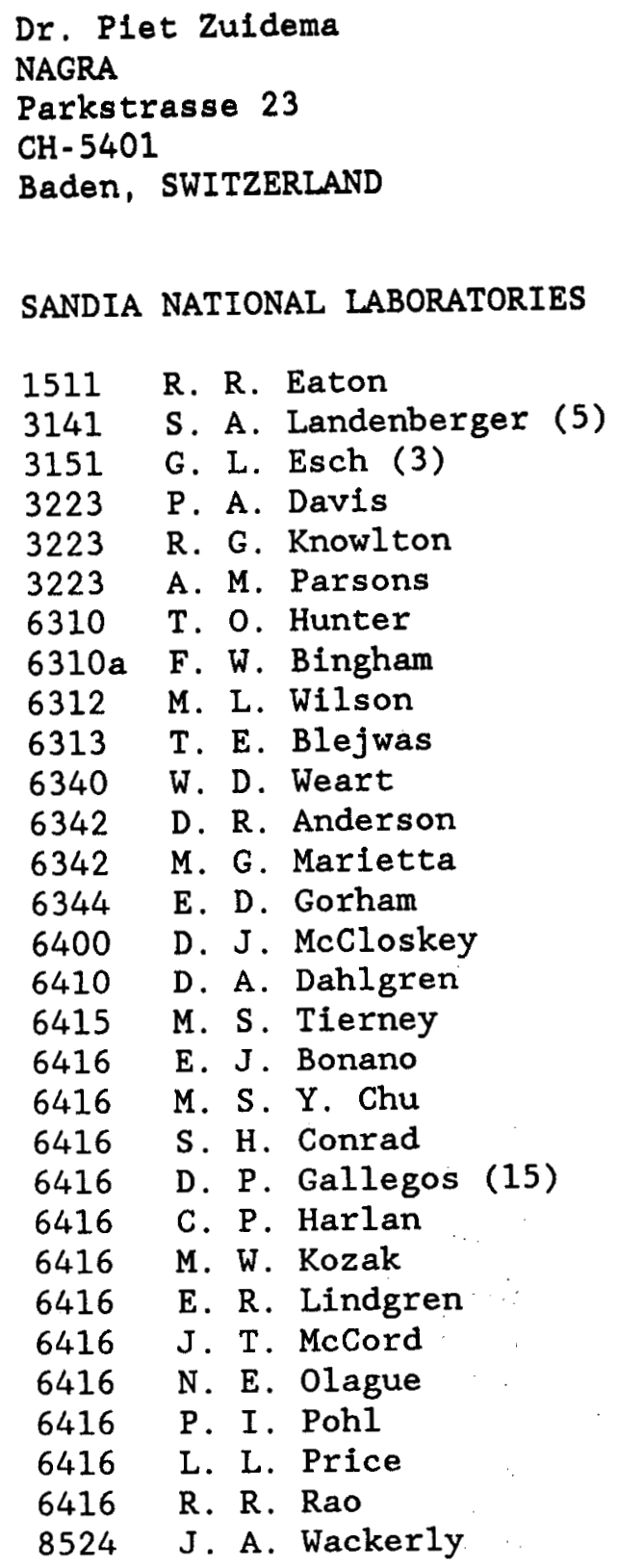

\section{DO NOT MICROFILM \\ THIS PAGE




\begin{tabular}{|c|c|}
\hline BIBLIOGRAPHIC DATA SHEET & \multirow{2}{*}{$\begin{array}{l}\text { 1. REPORT NUMBER } \\
\text { (Asslgned by NAC. Add Vol.. Supp.. Aev. } \\
\text { and Addendum Numbers, If any.) } \\
\text { NUREG /CR }-5536 \\
\text { SAND90-7015 }\end{array}$} \\
\hline \multirow{4}{*}{$\begin{array}{l}\text { 2. TITLE AND SUBTITLE } \\
\text { DCM3D, A Dual-Continuum, Three-Dimensional, Ground-Water } \\
\text { Flow Code for Unsaturated, Fractured, Porous Media }\end{array}$} & \\
\hline & 3. DATE REPORT PUBLISHED \\
\hline & February 1991 \\
\hline & 4. FIN OR $: R \triangle N$ AIELGER \\
\hline \multirow[t]{2}{*}{$\begin{array}{l}\text { 5. AUTHOR(S) } \\
\qquad \text { C.D. Updegraff*, C.E. Lee**, }\end{array}$} & 6. TYPE OF REPORT \\
\hline & 7. PERIOD COVERED (inctusive Datest \\
\hline \multicolumn{2}{|c|}{$\begin{array}{l}\text { 8. PERFORMING ORGANIZATION - NAME AND ADDRESS ("f NRC, provide Division, Office or Region, U.S. Nuclear Regulatory Commission, and mailing address: if contracior, grovide } \\
\text { norne and maiting address? }\end{array}$} \\
\hline $\begin{array}{lcl}* \text { *GAM, Inc. } & * * \text { Applied Physics, Inc. } & \text { Under } \\
1709 \text { Moon Street, NE } & 5353 \text { Wyoming Blvd., NE } & \text { Sandia } \\
\text { Albuquerque, NM } 87112 & \text { Albuquerque, NM } 87109 & \text { Albuque }\end{array}$ & $\begin{array}{l}\text { Under Contract to: } \\
\text { Sandia National Laboratories } \\
\text { Albuquerque, NM } 87185\end{array}$ \\
\hline \multicolumn{2}{|c|}{$\begin{array}{l}\text { 9. SPONSORING ORGANIZATION - NAME AND ADDRESS IINRC, trpe "Same as above": if contractor, provide NRC Division, Office or Region, U. S. Nuclear Regulatory Commission, } \\
\text { and mailing eddress.) } \\
\text { Officion of Engineering } \\
\text { U. S. Nuclear Regulatory Commission } \\
\text { Washington, DC } 20555\end{array}$} \\
\hline \multicolumn{2}{|c|}{ 10. SUPPLEMENTARY NOTES } \\
\hline \multicolumn{2}{|c|}{$\begin{array}{l}\text { 11. ABSTRACT } 1200 \text { wordorless } \\
\text { This report constitutes the user's manual for DCM3D. DCM3D is a computer code } \\
\text { for solving three-dimensional, ground-water flow problems in variably saturated, } \\
\text { fractured porous media. The code is based on a dual-continuum model with porous } \\
\text { media comprising one continuum and fractures comprising the other. The continua } \\
\text { are connected by a transfer term that depends on the unsaturated permeability of } \\
\text { the porous medium. An integrated finite-difference scheme is used to discretize } \\
\text { the governing equations in space. The time-dependent term is allowed to remain } \\
\text { continuous. The resulting set of ordinary differential equations (ODE's) is } \\
\text { solved with a general oDE solver, LsoDEs. The code is capable of handling } \\
\text { transient, spatially dependent source terms and boundary conditions. The boundary } \\
\text { conditions can be either prescribed head or prescribed flux. }\end{array}$} \\
\hline \multirow{5}{*}{$\begin{array}{l}\text { 12. KEY WORDS/DESCAIPTORS ILisi words or phrases" } \\
\text { computer code } \\
\text { dual continuum } \\
\text { fracture flow } \\
\text { ground water } \\
\text { unsaturated flow }\end{array}$} & 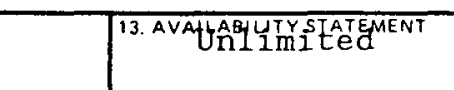 \\
\hline & $\begin{array}{l}\text { 14. SECURITY CLASSIFICATION } \\
\text { Trhis Pagel } \\
\text { UnClassified }\end{array}$ \\
\hline & $\begin{array}{l}\text { (This Report) } \\
\text { Unclassified }\end{array}$ \\
\hline & 15. NUMBER OF PAGES \\
\hline & \\
\hline
\end{tabular}


DO NOT MICROFILM
THIS PAGE

THIS DOCUMENT WAS PRINTED USING RECYCLED PAPER. 